\title{
FLORIAN LENK
}

DISTRIBUTION AND ECOLOGICAL

CHARACTERISTICS OF MEMBERS OF THE ROSEOBACTER GROUP 



\title{
DISTRIBUTION AND ECOLOGICAL CHARACTERISTICS OF MEMBERS OF THE ROSEOBACTER GROUP
}

\author{
Dissertation \\ for the award of the degree \\ Doctor rerum naturalium
}

at the Georg-August-Universität Göttingen

within the doctoral programme Biology

at the Georg-August University School of Science (GAUSS)

submitted by

FLORIAN LENK

from Ilmenau

Göttingen, 24/o6/2020 
THESIS ADVISORY COMMITTEE:

Prof. Dr. Rolf Daniel, Dept. of Genomic and Applied Microbiology, Faculty of Biology and Psychology

PD Dr. Michael Hoppert, Dept. of General Microbiology, Faculty of Biology and Psychology

MEMBERS OF THE EXAMINATION BOARD:

Prof. Dr. Rolf Daniel (Referee)

PD Dr. Michael Hoppert (Co-referee)

Prof. Dr. Kai Heimel, Dept. of Molecular Microbiology and Genetics, Faculty of Biology and Psychology

Prof. Dr. Stefanie Pöggeler, Dept. Genetics of Eukaryotic Microorganisms, Faculty of Biology and Psychology

Prof. Dr. Gerhard Braus, Dept. of Molecular Microbiology and Genetics, Faculty of Biology and Psychology

Prof. Dr. Jörg Stülke, Dept. of General Microbiology, Faculty of Biology and Psychology

DATE OF THE ORAL EXAMINATION:

$09 / 07 / 2020$ 
喫茶去

Drink tea. 

Marine Rhodobacteraceae are collectively referred to as the Roseobacter group, and due to their functional versatility and high abundance in diverse marine habitats, serve as model organisms in the study of microbial interactions, evolutionary processes, and metabolic pathways.

Two species within this group, which belong to the Octadecabacter genus, are of particular interest, as they were isolated from sea ice at both poles, an extreme habitat with regard to temperature, oxidative stress, and nutrient availability. Initial analyses demonstrated their unique position among roseobacters, since they contained an unusually large number of transposable elements, inferred gene duplications, and genome rearrangements. However, a lack of comparable genome sequences from closely related strains left unclear, to what extent these observations are actual evidence of environmental adaptations, and how their genomic features compare to those of temperate strains in the same genus.

This thesis expanded the pool of available Octadecabacter genome sequences, and utilised the additional data to examine general genomic properties and the nature of extreme adaptation in this group. Using phylogenomic methods, the phylogenetic history of the Octadecabacter-associated strains was reconstructed and evaluated in the wider context of the Roseobacter group. Comparative gene content analyses were applied to illustrate which aspects of cellular metabolism and biochemistry are altered in polar Octadecabacters, and were put into an evolutionary perspective utilising a model of functional gene content evolution. In addition, the global distribution of individual subgroups within the Octadecabacters was examined by means of a metagenomic mapping approach.

The analyses presented here demonstrate that genomes of polar Octadecabacters encode more complex metabolic networks, consistent with a broader spectrum of available nutrients and more diverse microbial interactions in sea ice. Genome flexibility, and evolvability in general, constitute important prerequisites for efficient adaptation to this extreme habitat, and are both more pronounced in polar than in temperate Octadecabacter genomes. Detection patterns of Octadecabacters in metagenome sequences suggest that the currently available polar isolates are members of a cosmopolitan genus that also features non-polar species.

The presented results add to our knowledge of the nature of extreme adaptation and its potential underlying processes in roseobacters, and are a contribution to our goal of understanding the biogeography of this important marine group. 

It is no understatement that I could not have finished this thesis without the support of my friends and family, whom I want to thank at this place. First, I want to deeply thank John Vollmers and Andreas Leimbach. The role which your support has played during these years cannot be overstated. Thank you, Andreas, for promoting an open and cooperative form of science, and for sharing your knowledge and experience so that it may benefit others. And thank you, John, for many fruitful discussions, and for your critical input, without which this thesis simply would not be what it is today. Thank you both for your selflessness, for always coming to the help of others without hesitation, and for the sense of community you create around you!

Speaking of community, I want to thank the group's former Ph.D. students, including, but not limited to, Marvin, Chris, and Sascha, for many cheerful hours and interesting conversations. Sascha should be especially thanked for his competent work and support as local sysadmin, since he helped provide the tools for my scientific work and that of others, (mostly) without complaining. Only when I had to be my own sysadmin did I find out, which nuisances this job can hold ready...

We should never forget that we, intentionally or not, shape the future scientific community through the way we treat young academics. Unfortunately, caring for anyone but oneself is poorly incentivised by our current system. Over the years, I had the pleasure of supervising two students during their bachelor thesis. I am grateful for the opportunity to take part in their education, and hope that I could show both that we, as agents, are not bound by the incentives set by the system, and that compassion is better than complacency. Karin and Annika, I wish you nothing but the best for your future.

I would also like to thank my friends outside of the lab, with whom I sometimes found emotional support, but always found fun.

With all the support I received from my friends, none of it would have mattered were it not for my mother. When, after just over three years, I was left without funding for my thesis, she stepped in and privately funded me for the remainder of its duration. This amount of compassion and endurance, after having single-handedly raised two sons - one of them with a disability - speaks of a stronger character than most other people possess, and is deserving of anyone's respect. Thank you, mom!

I also want to thank the community for providing a framework that makes theses such as mine possible in the first place: thank you to the people, who create and maintain open and free resources teaching 
people how to program, analyse data, and so much more; thank you, residents of Stack Overflow, for spending your private time to help solve problems of others; and thank you, members of my thesis committee and examination board, for taking the time to read and evaluate my work!

My final thank-you goes out to my little friend, because he always kept my feet warm during long nights. $\odot$

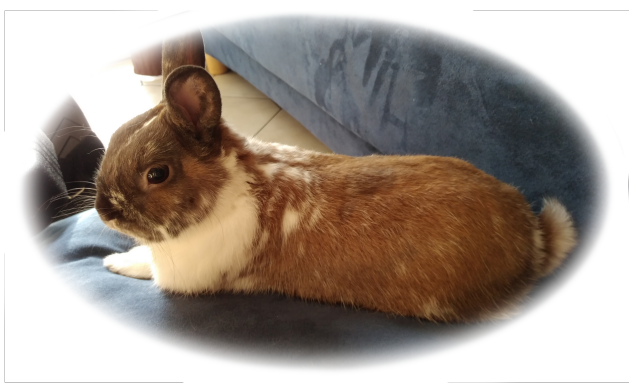




\section{CONTENTS}

1 INTRODUCTION 1

1.1 Microbial life in the ocean 2

1.2 Life in cold habitats 3

1.3 The Roseobacter group 6

1.4 The Octadecabacter genus 7

1.5 Rationale 9

2 MATERIAL AND METHODS 13

2.1 Genome sequencing 13

2.2 Genome assembly, annotation, and statistics 14

2.3 Sequence comparison of Octadecabacter clade genomes 14

2.4 Bacterial genomes used for comparative analysis 14

2.5 Orthologue detection 20

2.6 Roseobacter group phylogeny reconstruction 20

2.7 Octadecabacter clade phylogeny and gene content clustering 21

2.8 COG annotation 22

2.9 Birth-and-death model of functional gene content evolution 22

2.10 Gene content analyses 23

2.10.1 Pan- and core-genome calculations 23

2.10.2 Determination of group-specific orthologous groups 23

2.11 Protein secondary structure prediction and comparison 23

2.12 Octadecabacter occurrence in metagenomes 24 2.12.1 Selection of metagenomes 25

2.12.2 Read mapping 25

2.12.3 Filtering and normalisation 25

3 RESULTS 27

3.1 Genome assembly and general comparison 27

3.2 Phylogeny 29

3.3 General gene content 32

3.3.1 Pan-genome analysis 32

3.3.2 Distribution of COG categories 35

3.4 Group-specific genes 38

3.4.1 Genes specific to polar Octadecabacters 39

3.4.2 Genes specific to temperate Octadecabacter isolates 51

3.4.3 Genes specific to Pseudooctadecabacter-related genomes

3.5 Functional gene content evolution 53

3.5.1 Thiamine metabolism 58

3.5.2 Tetrapyrrole biosynthesis 61

3.5.3 Methionine metabolism 61 
3.5.4 Histidine degradation 63

3.5.5 General cellular stress response 63

3.5.6 Other metabolism-related functions 65

3.6 Paralogue occurrence in Octadecabacter clade members 66

3.7 Key characteristics in polar roseobacters $\quad 67$

3.8 Protein secondary structure content 70

3.9 Occurrence of Octadecabacter-related sequences in metagenomes

4 DIsCUSSION 81

4.1 Octadecabacter lifestyle and gene content divergence 81

4.1.1 Phylogeny 81

4.1.2 Pan-genome analysis 87

4.1.3 Genus delineation within the Octadecabacter clade 90

4.2 Functional gene content and its evolution 93

4.2.1 General trends in COG content 93

4.2.2 Properties of the birth-and-death model 98

4.3 Environmental adaptations in polar Octadecabacters 100

4.3.1 Genomic adaptation mechanisms 101

4.3.2 Metabolic properties of polar Octadecabacters 103

4.3.3 Genomic indicators of psychrotrophic adaptations 108

4.3.4 Comparison to other polar members of the Roseobacter group 113

4.4 Global distribution of Octadecabacter-related sequences 115

5 CONCLUSION 119

A APPENDIX 123

A.1 Supplementary files 123

A.2 Organism metadata 125

A.3 Roseobacter group phylogeny and other trees 131

A.4 Group-specific genes 135

A.5 Xanthorhodopsins in Roseobacter group bacteria 167

A.6 Phylogenetic birth-and-death model 169

A.7 Read coverage of PAMC 27224 contigs 203 


\section{LIST OF FIGURES}

Figure 1.1 Isolation locations of Octadecabacter clade strains 8

$\begin{array}{lll}\text { Figure 3.1 Digital DDH of Octadecabacter clade genomes } 28 & 28\end{array}$

Figure 3.2 Octadecabacter- and neighbouring clade phylogeny 30

Figure 3.3 Phylogeny versus gene content in the Octadecabacter clade $\quad 32$

Figure 3.4 Octadecabacter clade pan-and core-genome plots

Figure 3.5 Roseobacter group pan-and core-genome plots 34

Figure 3.6 Octadecabacter clade core-, cloud-, and shellgenome sizes 35

Figure 3.7 COG category distributions in the Octadecabacter clade $\quad 36$

Figure 3.8 Numbers of group-specific genes 39

Figure 3.9 MarR and associated genes specific to polar Octadecabacters 40

Figure 3.10 AAA ATPase specific to polar Octadecabacters 41

Figure 3.11 Selenium-binding protein specific to polar Octadecabacters $\quad 42$

Figure 3.12 Xanthorhodopsin gene cluster in polar Octadecabacters $\quad 42$

Figure 3.13 Genes associated with carbohydrate uptake and degradation specific to polar Octadecabacters

Figure 3.14 RpiR and associated genes specific to polar Octadecabacters 45

Figure 3.15 Polar Octadecabacter-specific genes for degradation of diverse substrates 47

Figure 3.16 $m t n A$ and $f u c A$ in polar Octadecabacters 48

Figure 3.17 DNA polymerase V in polar Octadecabacters 48

Figure 3.18 Dehydrogenase gene cluster specific to polar Octadecabacters 49

Figure 3.19 CounT model parameters within the Octadecabacter clade 59

Figure 3.20 Key figure comparison in polar roseobacters 69

Figure 3.21 Hierarchical clustering of Octadecabacters based on protein secondary structure $\quad 70$

Figure 3.22 Statistical differences in secondary structure content 71

Figure 3.23 Amino acid frequencies in Octadecabacter groups $\quad 72$ Figure 3.24 $\alpha$-Helix amino acid content in Octadecabacter groups 73 
Figure 3.25

Figure 3.26

Figure 3.27

Figure 4.1

Figure 4.2

Figure 4.3

Figure 4.4

Figure 4.5

Figure 4.6

Figure 4.7

Figure 4.8

Figure 4.9

Figure 4.10

Figure 4.11

Figure A.1

Figure A.2

Figure A.3

Figure A.4

Figure A.5

Figure A.6

Figure A.7

Figure A.8

Figure A.9
Octadecabacter groups at metagenome sampling sites $\quad 74$

Metagenomes ranked by abundance of Octadecabacter groups 75

Frequency of Octadecabacter group occurrence in different biomes $\quad 79$

IC and TC support of Octadecabacter clade and neighbouring bipartitions $\quad 82$

RF distances between Roseobacter group core gene phylogenies 84

Phylogeny versus gene content in the Roseobacter group 86

Pan-genome size change in Roseobacter group lineages $\quad 88$

Singleton genes in Roseobacter group genomes $\quad 89$ Genome category fractions among selected Roseobacter group lineages 90

Nucleotide identity within Octadecabacter groups POCP versus $16 \mathrm{~S}$ rRNA gene similarity in roseobacters 92 Changes in COG categories D, F, J, and L along the Octadecabacter lineage 94

Changes in COG categories C, G, N, and X along the Octadecabacter lineage $\quad 97$

Changes in COG categories $\mathrm{H}, \mathrm{K}, \mathrm{R}$, and $\mathrm{S}$ along the Octadecabacter lineage $\quad 99$

Folder structure of supplementary data files 123

MLSA phylogeny of the Roseobacter group 131

Phylogeny versus core-reduced gene content in the Roseobacter group 133

Hierarchical clustering of genomes based on geographic distance 134 Isolation points of xanthorhodopsin-bearing roseobacters 167

Xanthorhodopsin phylogeny $\quad 168$

Lineage-specific CounT model parameters 169

Overview of tables listing COG changes along birth-and-death model phylogeny 172

Read coverage of isolate PAMC 27224 contigs 203

LIST OF TABLES

Table 2.1 
Table 2.2

Table 3.1

Table 3.2

Table $3 \cdot 3$

Table $3 \cdot 4$

Table 3.5

Table 3.6

Table $3 \cdot 7$

Table A.1

Table A.2

Table A.3

Table A.4

Table A.5

Table A.6

Table A.7

Table A.8

Table A.9

Table A.10

Table A.11
Genomes for comparative analysis 15

General Octadecabacter clade genome features

Roseobacter group lineages branching at similar root distance as the Octadecabacters 31

Pan- and core-genome parameters within Roseobacter group members 33 COG category trends in polar Octadecabacters COG gains and losses in LCA of polar Octadecabacters 54

Number of paralogues in Octadecabacter clade genomes $\quad 67$

Polar Roseobacter group isolates and their associated clades 68

Organism metadata 125

Group-specific genes: group I 135

Group-specific genes: group II 138

Group-specific genes: group III 140

Group-specific genes: group IV 142

COG changes at Octadecabacter clade LCA 173 COG changes at LCA of polar Loktanella isolates 173

COG changes at ancestor of Loktanella fryxellensis 183

COG changes at LCA of Loktanella vestfoldensis strains 184

COG changes at LCA of O. temperatus and O. ascidiaceicola 192

COG changes at LCA of Antarctic Octadecabacter isolates 200

\section{ACRONYMS}

$\begin{array}{ll}\text { 3-MST } & \text { 3-mercaptopyruvate sulfurtransferase } \\ \alpha \text {-KG } & \alpha \text {-ketoglutarate } \\ \text { AAP } & \text { aerobic anoxygenic photosynthesis } \\ \text { Ac-CoA } & \text { acetyl-CoA } \\ \text { AFP } & \text { antifreeze protein } \\ \text { AMP } & \text { adenosine monophosphate } \\ \text { ANI } & \text { average nucleotide identity }\end{array}$


API application programming interface

ATP adenosine triphosphate

BLAST basic local alignment search tool

bp base pair

cAMP cyclic AMP

CDD Conserved Domains Database

CDS coding DNA sequence

CoA coenzyme A

COG cluster of orthologous groups

CSP cold-shock protein

DDH DNA-DNA hybridisation

DHA dihydroxyacetone

DHAP DHA phosphate

DHPS 2,3-dihydroxypropane-1-sulfonate

DNA desoxyribonucleic acid

DOC dissolved organic carbon

DOM dissolved organic matter

DSMZ Deutsche Sammlung von Mikroorganismen und Zellkulturen

DUF domain of unknown function

EPS extracellular polysaccharides

ete3 Environment for (phylogenetic) Tree Exploration

FDR false discovery rate

GAPDH glyceraldehyde-3-phosphate dehydrogenase

GB GenBank

GGDC genome-to-genome distance calculator

GPDH glycerol-3-phosphate dehydrogenase

GS glutamine synthetase

GTA gene transfer agent 
HFB high-flux backbone

HMP hydroxymethyl pyrimidine

IC internode certainty

IMG Integrated Microbial Genomes

IS insertion sequence

KEGG Kyoto Encyclopedia of Genes and Genomes

KO KEGG orthology

LCA latest common ancestor

LGT lateral gene transfer

MB marine broth

Mbp mega base pairs ( $\left.10^{6} \mathrm{bp}\right)$

mg-RAST RAST for metagenomes

ML maximum likelihood

MLSA multilocus sequence analysis

MP maximum parsimony

MTA methylthioadenosine

MTHF methyl-tetrahydrofolate

MTR methylthioribose

MUSCLE multiple sequence comparison by log-expectation

NAD nicotinamide adenine dinucleotide

NCBI National Center for Biotechnology Information

NGS next-generation sequencing

NJ neighbour-joining

OG orthologous group

PAMC Polar and Alpine Microbial Collection

$\mathrm{pH} \quad$ pondus hydrogenii

POCP percentage of conserved proteins

POM particulate organic matter

PSSM position-specific scoring matrix 
RAST Rapid Annotation using Subsystems Technology

$\mathrm{RF} \quad$ Robinson-Foulds (distance)

RNA ribonucleic acid

rpsBLAST reverse position-specific BLAST

rRNA ribosomal RNA

SAM S-adenosylmethionine

SBP Selenium-binding protein

SD standard deviation

SIMCO sea ice microbial community

SOP standard operating procedure

TC tree certainty

TCA tricarboxylic acid

TE transposable element

TLS translesion DNA synthesis

TRAP tripartite ATP-independent periplasmic (transporter)

tRNA transfer RNA

Usp universal stress protein

UV ultraviolet 
The global ocean covers just under $71 \%$ of Earth's surface. With an estimated volume of $1.332,4 \times 10^{21} \mathrm{~L}$ [1], it constitutes our planet's largest consecutive habitat. Its role in climate regulation and nutrient cycling is well established, yet poorly understood. For example, $86 \%$ and $76 \%$ of total global evaporation and precipitation, respectively, take place over the ocean $[2,3]$, making it the global water cycle's most important component. Nonetheless, for vast oceanic regions, we still lack sufficient flux data to accurately model the profound influence on biogeochemistry and how they are, in turn, influenced by global climate change [4]. Furthermore, the ocean constitutes a major carbon sink, with an estimated $50 \%$ of all non-fossil organic carbon stored in oceanic environments, mostly in the form of dissolved organic carbon (DOC), and in marine sediments $\left(700 \times 10^{9}\right.$ tons and $1,750 \times 10^{9}$ tons, respectively) $[5,6]$. Each year, marine phytoplankton converts $50 \times 10^{9}$ tons of inorganic to organic carbon, accounting for about half of the world's primary production [7]. Yet, we have only recently begun to unravel the complex interactions and interrelations between these diverse carbon pools and to map out the role microbes play in this cycle [8]. Particularly the latter aspect is of general interest, considering that even sea water with severely limited nutrient content contains $10^{4}$ bacterial cells $/ \mathrm{mL}$, and that the estimated average cell density in the oceans is tenfold this value [9]. It is now widely recognised that microbiota play a decisive role in shaping the marine and terrestrial environments. In order to understand the system as a whole, we need to understand the complex metabolic networks and mutual interactions between its microbial components.

The recent advent of next-generation sequencing (NGS) methods (e. g. $[10,11])$ has given us unprecedented capabilities to address this topic in detail [12]. With the availability of an ever-increasing number of bacterial genome sequences, it became apparent that several genomic features can be linked to specific niches in marine habitats [13-15]. Furthermore, cultivation-independent meta-omics techniques enable the detailed mapping of such genomic features to system dynamics on the community level and beyond $[12,16]$. The following chapter will briefly summarise our current understanding of the prokaryotes' role in marine ecosystems. 
Considering the total biomass of oceanic microbes, their importance in shaping the biochemistry of marine habitats becomes apparent. As noted above, primary production adds approximately $50 \times 10^{9}$ tons of organic carbon annually to the marine food web, mainly by the action of Dinoflagellates, Diatoms, and Cyanobacteria. Since the only other major carbon input sources, atmospheric deposition and riverine input, contribute an estimated $0.2 \times 10^{9}$ tons $\mathrm{C} /$ year each [17], it is obvious that the life of heterotrophic organisms, which require carbon compounds for their growth, is centred around areas of primary production. Photosynthesis takes place at the ocean surface layer, down to depths of $\approx 100 \mathrm{~m}$ where about $1 \%$ of incident light remains, sometimes summarised as the photic zone. Overall, relatively warm surface waters of the photic zone constitute only approximately $2 \%$ of the ocean volume, whereas the vast majority, $80 \%$, is cold deep water [17], which has a highly uniform temperature range of around o to $3{ }^{\circ} \mathrm{C}$. Here, only little primary production takes place (in the form of chemoautotrophic carbon fixation $[18,19])$ and respiratory processes dominate. Nutrients are mainly gained by remineralising the more labile compounds of sinking particulate organic matter ( $\mathrm{POM})$. As a consequence and since the respiratory processes are slow, cold deep water is enriched in nutrients compared to the surface water, where most compounds available through primary production are quickly recycled.

Apart from carbon, microbial growth capacities are mostly dictated by the availability of phosphorus, nitrogen and iron, the latter two of which are usually limiting factors in oligotrophic sea water [20]. Reactive nitrogen input - primarily in the form of oxidised nitrogen species, $\mathrm{NO}_{x}-$ is in the order of $20 \times 10^{6}$ tons/year from rivers and $67 \times 10^{6}$ tons/year from the atmosphere $[17,21]$. Notably atmospheric nitrogen deposition has increased drastically through human activity since the beginning of industrialisation [21]. A similar amount, $100 \times 10^{6}$ tons, is deposited annually in the ocean environment through microbial $\mathrm{N}_{2}$-fixation [21].

As indicated above, the distribution of these nutrients varies throughout different water masses. In tropical waters, high levels of solar irradiation increase the water temperature and, due to evaporation, its salinity. This leads to the formation of a strong thermocline and pycnocline, which prevent mixing of the surface and its subjacent layers, and thereby stratify the water [17]. Due to the constant export of nutrients through sedimentation of POM, the surface waters constitute a largely nutrient-replete, oligotrophic habitat [17]. At high latitudes, where solar irradiation is weaker, the thermo- and pycnocline are mostly absent [17]. Here, surface waters are cooled down by the cold air and start to sink, thereby pushing the underlying water to greater depths. These water masses then flow along the sea bed, following the earth's topol- 
ogy until they resurface at specific points, e.g. the North Pacific [17]. In combination with warmer, wind-driven surface currents, a globalscale circulation of water results, called the thermohaline circulation, which is the most important factor in nutrient transport within the marine environment [17].

Thus, microbiota are main contributors to the influx of nutrients into the marine system, whereas geochemical processes in the form of water currents determine their distribution and thereby set the general living conditions for the microbial residents. These conditions vary regionally. For example, while surface waters are usually oligotrophic, upwelling of cold, nutrient-rich deep water can locally increase the amount of available solutes and dissolved organic matter (DOM). Marine sediments and estuaries present additional habitats, which constitute important sources of some nutrients, and sinks for others [17]. Furthermore, the marine environment also includes more extreme niches such as sea ice, or deep sea vents. This variety of habitats predisposes marine bacteria to a large phylogenetic and functional diversity, encompassing both cosmopolitan groups with a world-wide distribution, as well as highly specialised endemic species.

The two most abundant and ubiquitous bacterial groups are Prochlorococcus (Cyanobacteria) and Pelagibacter ubique ( $\alpha$-Proteobacteria) [22]. Both are adapted to oligotrophic growth conditions, exhibiting small cell sizes and highly streamlined genomes $[22,23]$. In contrast, marine vibrios ( $\gamma$-Proteobacteria), and members of the Roseobacter group ( $\alpha$ Proteobacteria) usually possess larger genomes, and generally follow a more copiotrophic life style. Their representatives often live in close association with primary producers $[22,24]$. Roseobacters in particular demonstrate a degree of genomic flexibility that lets them functionally adapt to diverse ecological niches (discussed in more detail in a later section).

Such genomic adaptations and their underlying mechanisms are of great scientific interest, both for understanding evolutionary processes, as well as mapping out the factors shaping marine ecology [12, 25]. This thesis will contribute by investigating the adaptation of a group of organisms to a cold and otherwise extreme habitat.

Since cold habitats are the most expansive in the marine environment and pose specific restrictions for their resident microorganisms, their properties and general microbial adaptations will be elucidated in the following chapter.

\subsection{LIFE IN COLD HABITATS}

The low temperature in habitats of the cryosphere has a direct effect on several aspects of cellular biochemistry, which cells need to address in order to maintain viability. The first is membrane fluidity: at lower temperatures, the lipid bi-layer is in danger of transitioning from a 
liquid-crystalline into a gel phase, which ultimately leads to loss of its function. Cells combat this transition by incorporating shorter, as well as (poly-)unsaturated fatty acids, among other modifications [26]. A high number of fatty acid desaturase genes has therefore been associated with cold adaptation in some bacterial genera [15].

Secondly, reaction rates drop exponentially with decreasing temperature [27], and in order to maintain proper function, enzymes undergo distinct changes in their structure and composition. In general, cells seem to trade enzymatic stability for activity at lower temperatures [27], mainly by reducing intra-molecular interactions in the protein core, and by increasing the solvent interactions at its surface [28]. The concomitant change in amino acid composition overall includes an increase in glycine residues, and a decrease in proline content [28]. Since secondary structure elements are crucial for a protein's tertiary structure and general function, it is thought that their content does not differ drastically from mesophilic to cold-adapted enzymes, although structural data indicate that more destabilising amino acids are incorporated into $\alpha$-helices of the latter [29].

The mentioned alterations also serve to facilitate correct protein folding at low temperatures. Nonetheless, some organisms also require dedicated cold-adapted chaperones for viability [28].

In addition, cells need to counter the stabilising effect cold has on secondary structures of both desoxyribonucleic acid (DNA) and ribonucleic acid (RNA) molecules, in order to maintain function of the transcriptional and translational machineries. A universal tactic to that end is the expression of cold-shock proteins (CSPs), which bind single stranded DNA and RNA, and thus suppress the formation of secondary structures [30]. Psychrophilic organisms furthermore incorporate more dihydrouridine into transfer RNA (tRNA) molecules than their mesophilic relatives, thereby increasing their flexibility [31]. Translational efficiency is also maintained by the expression of specific accessory proteins to the ribosome in some bacteria $[32,33]$.

Since temperatures in habitats of the cryosphere are near or below the freezing point of water, microbes need to prevent ice crystal formation in order to maintain viability. One tactic is to lower the freezing point through the accumulation of solutes and ions [34], which is why most of the isolated and described psychrophilic organisms show similarities to halophilic or halotolerant individuals. Frequently produced compatible solutes include glycerol, glycine-betaine, and trehalose, among others [34, 35]. Moreover, many organisms express antifreeze proteins (AFPs), which control the crystallisation of water and thereby avoid physical damage to the cellular environment [36]. Cold-dwelling microorganisms also often produce extracellular polysaccharides (EPS) in order to decrease the freeze point in the extracellular space [37]. Particularly in sea ice, Diatoms produce high amounts of EPS for cryoprotection [38]. 
A further effect of low temperature is the higher solubility of oxygen, which therefore exposes cells to higher levels of oxidative stress. To counter this, the specific genetic equipment of psychrophiles often contains more oxygen-consuming enzymes [15, 39], as well as functions to detoxify $\mathrm{O}_{2}^{-}$radicals like dismutases, catalases, and others $[15,40]$.

As mentioned in the previous section, deep ocean water is the single most extensive low-temperature habitat. Sea ice, on the other hand, covers a comparatively small surface $\left(\approx 15 \times 10^{6}\right.$ to $\left.22 \times 10^{6} \mathrm{~km}^{2}[41]\right)$, but still harbours dense and highly diverse microbial communities [42]. Because of its relevance for this thesis, its genesis and characteristics as a microbial habitat will be elaborated in more detail below.

Sea ice builds up when a uniform layer of ice crystals forms undisturbed at the air-water interface, and these crystals grow downward due to the continued extraction of heat from the water below (congelation ice) [41]. If strong winds mix the upper water layer, small ice crystals form in the mixed layer, and rise to the surface once mixing stops, where they then grow and form pancake ice [41]. In both cases, ions and solutes contained in the freezing sea water are extruded and collect between the ice crystals in brine channels, pockets of liquid water in which salinity can reach near-saturation levels. From these channels, brine flows back into the sea until the channels become disconnected, which decreases the overall salinity of the ice [41]. Notably, sea ice microorganisms are known to hinder this flow via the production of EPS [43]. During the summer months, the ice melts at the surface due to increased solar irradiation. The melted water can flow back down into the sea through channels in the ice, and in the process flush out nutrients and the resident microorganisms [44]. Thus, sea ice bacteria encounter osmotic stress in the form of phases of both extremely high and low salinity, and can also face expulsion from their habitat. While oxygen solubility is decreased in sea ice brine due to the high salt concentration [45], both poles show seasonally increased levels of heavy metals [46-48], which constitute sources of significant oxidative stress for microorganisms (e.g. [49]). Ultraviolet (UV) radiation likewise varies seasonally, and can reach high intensities at the poles [50], posing another form of stress.

Nonetheless, sea ice is a highly productive habitat, which constitutes the basis of the local marine food web and harbours multiple trophic levels $[41,42]$. The densest microbial populations form at the ice-sea interface, where the temperature remains mostly uniform at around $-2{ }^{\circ} \mathrm{C}$ [44]. This community is called the sea ice microbial community (SIMCO) and contains Diatoms as the main primary producers. The numerically dominant prokaryotic groups are Flavobacteria (mostly the genera Psychrobacter and Polaribacter), and $\gamma$-Proteobacteria (Marinobacter, Glaciecola and Colwellia) [42,51], which live in close association with the primary producers. A high availability of nutrients for bacte- 
ria is reflected in the fact that a comparatively large fraction of bacteria from sea ice is readily cultivable [51].

$\alpha$-Proteobacteria are often less abundant in sea ice than in the underlying sea water $[52,53]$. However, they can make up a significant portion of the SIMCO and benefit their Diatom hosts [54]. The most abundant genus are usually Octadecabacters [51], which belong to the Roseobacter group. Although present in cold and otherwise extreme habitats $[55,56]$, this group of organisms often dominates mesophilic marine communities [24]. It will be described in the following chapter.

\subsection{THE ROSEOBACTER GROUP}

Almost thirty years ago, Shiba instituted the Roseobacter genus within the $\alpha$-Proteobacteria, whose members were characterised by their ability to produce bacteriochlorophyll under aerobic conditions [57]. Since then, this feature has been termed aerobic anoxygenic photosynthesis (AAP) [58], and a growing number of related bacterial strains has been isolated from a variety of environments, together called the Roseobacter group.

In general, all marine Rhodobacteraceae should be considered roseobacters [59]. Initially, these were thought to form a monophyletic clade, and earlier reconstructions of this group's phylogeny defined five subclades, which subsequently served as the basis for multiple comparative analyses (e. g. [60, 61]). This view was however updated recently by Simon et al., who showed the roseobacters to be paraphyletic to nonassociated Rhodobacteraceae, and that a terrestrial life style has evolved multiple times in the Roseobacter group phylogeny [59]. It should be stressed that the term roseobacter does not constitute a valid taxonomic unit above the genus level. Simon et al. further re-categorised this group into a set of eleven prevalent clades, based on a larger number of comparison genomes, as well as stricter criteria of phylogenetic robustness [59].

The roseobacter lineage likely emerged $\approx 250$ million years ago, coinciding with the radiation of marine Dinoflagellates [62, 63]. Its members are often found in association with eukaryotic hosts, and have thus emerged as model organisms for the study of the relating interactions $[64,65]$. Consequently, roseobacters are highly abundant in coastal regions and during algal blooms [66]. However, they also occupy a wide range of other marine habitats [24], and thus, due to their wide occurrence, play an important role in shaping the ocean's biogeochemistry (with a potentially strong role in global sulphur cycling [67, 68]). This is why this group has received increased attention in recent years, and why efforts have been made to isolate and sequence more of its members from diverse environments.

As far as we can tell from currently sequenced representatives, Roseobacter group bacteria predominantly seem to maintain comparatively 
large and flexible genomes, which provide them the metabolic versatility to occupy diverse niches [69, 70]. This tactic stands in contrast to, e.g. Prochlorococcus and Pelagibacter, which tend towards small and highly streamlined genomes that are optimised to grow in oligotrophic waters, and which numerically dominate this type of habitat [69].

An important contribution to the roseobacters' genomic flexibility are gene transfer agents (GTAs), small, virus-like particles, which pack and transmit fragments of the host's DNA [71]. Earlier investigations found these agents in the vast majority of roseobacter genomes and demonstrated their activity in vivo [72].

This flexibility makes this group of organisms ideal to study the evolution and regulation of specific physiological and metabolic features, and relate these to the adaptation to new habitats where appropriate. Examples of features which have already been analysed in some detail are the degradation of aromatic compounds [73], flagellum gene clusters [74], AAP [75], or genomic adaptations to life in surface ocean water [13].

Besides their high abundance in coastal regions and during algal blooms, the Roseobacter group is also present at a number of more extreme habitats, such as sea ice [76], or Antarctic hypersaline lakes [55]. One genus, which harbours multiple isolates from extreme and cold environments, is the Octadecabacter genus. Its representatives therefore lend themselves to study extremophilic and psychrophilic adaptation within the Roseobacter group, and the following chapter will give a brief overview of this genus and its associated strains.

\subsection{THE OCTADECABACTER GENUS}

Gosink, Herwig, and Staley isolated the first members from Arctic and Antarctic sea ice samples, and named them Octadecabacter arcticus and O. antarcticus, respectively [76]. They chose the genus name based on the major fatty acid present in these bacteria, which is octadecenoic acid. These species attracted scientific interest at the time, due to their strictly psychrophilic life style, as well as their high sequence similarity despite the large geographic distance. Consequently, their genomes were sequenced and analysed in order to identify common features, as well as functional differences which might be linked to the respective habitats $[61,77]$. However, a lack of genomes suitable for comparison reduced the generality of these findings and it remained unclear, in how far functions related to adaptation to life at the poles really are specific (i. e. exclusive) to polar Octadecabacter species [77].

In recent years, a number of Rhodobacteraceae were isolated from temperate habitats, which, based on their $16 \mathrm{~S}$ ribosomal RNA (rRNA) sequences, were assigned to the Octadecabacter genus. The first of these described in more detail was O. jejudonensis, originating from an estuary of Jeju island, South Korea [78] (see Figure 1.1 for an overview of the 


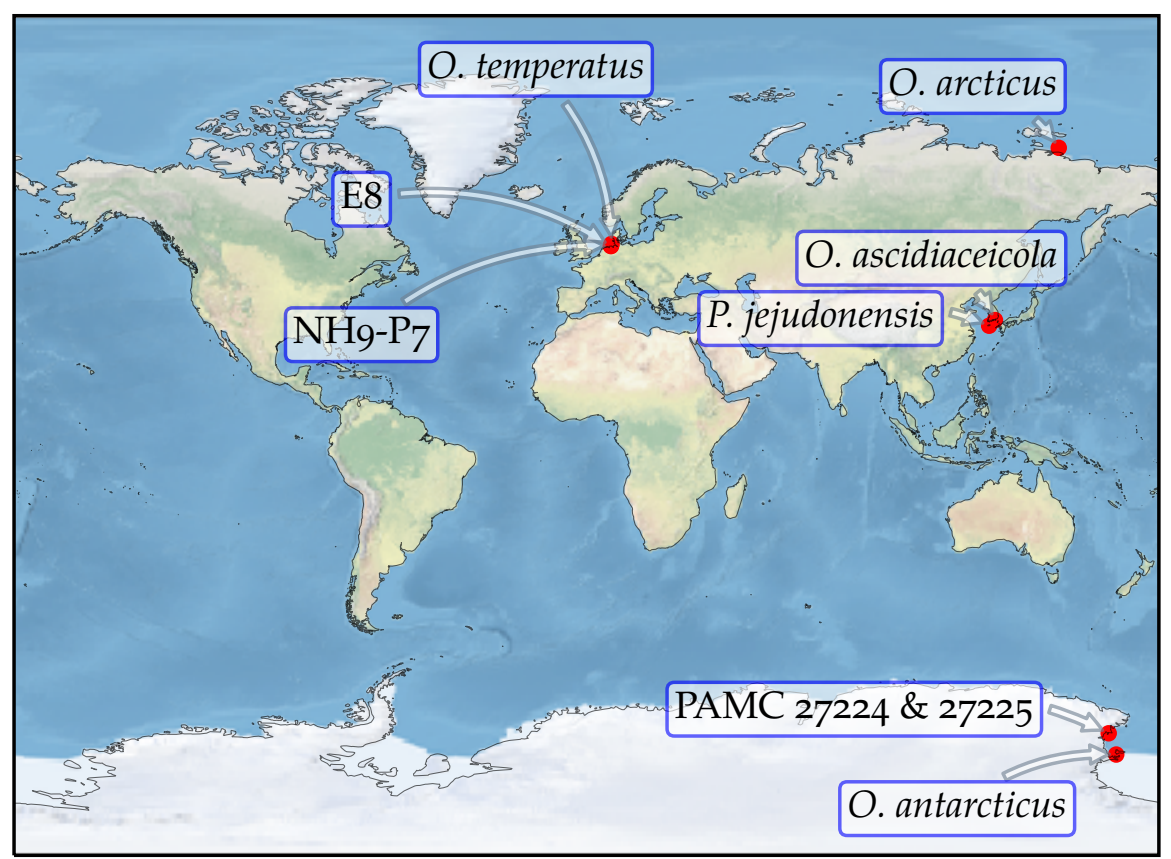

Figure 1.1: The isolation points of Octadecabacter-related strains relevant for this thesis.

isolation coordinates of all Octadecabacter strains relevant to this study). Other temperate isolates followed soon after, most notably O. temperatus, isolated from sea water of the North Sea [79]. In their polyphasic analysis, Billerbeck et al. proposed the reclassification of O. jejudonensis, and the institution of a novel genus Pseudooctadecabacter, due to its large differences to the polar Octadecabacters and temperate O. temperatus [79]. Further polar isolates were recently obtained from Antarctic sediment, which were assigned to the $O$. antarcticus species, based on $16 \mathrm{~S}$ rRNA sequence similarity [80]. Both were deposited at the Polar and Alpine Microbial Collection (PAMC), and initial studies showed that they are psychrotolerant, rather than stenopsychrophiles like the two polar type strains.

Since the polar Octadecabacters dwell in one of the most extreme marine-associated habitats known, studying them may prove invaluable for understanding adaptation to extreme environments in the Roseobacter group in general. The availability of four polar isolates constitutes a good basis for genomic comparisons and to test the specificity of observed traits. Besides O. temperatus and P. jejudonensis, further temperate isolates were recently acquired and their genomes sequenced: Octadecabacter sp. NHy-P7 was isolated from an Oyster shell, and Octadecabacter sp. E8 from sediment, both in the North Sea (Figure 1.1, both unpublished). In addition, the type strain of the new species $O$. ascidiaceicola [81] was genome-sequenced. This strain was isolated from the sea squirt Halocynthia roretzi [81]. 
Taken together, a group of nine closely related strains was available for comparison, of which the genomes of O. ascidiaceicola, P. jejudonensis, and the PAMC isolates had to be sequenced during this thesis. It should be noted that other cultivated strains exist, which have been classified as Octadecabacters, e. g. the recently described O. ponticola [82]. The nine strains introduced here (and marked in Figure 1.1) were, however, the only ones available during the time of this thesis' experimental phase, and are consequently the only ones analysed in more detail.

The new isolates' association to the Octadecabacter genus relied solely on their 16S rRNA sequences. Such comparisons are, however, associated with great uncertainty [83]. From the analyses of Billerbeck et al. [79], it is already clear that the nine strains described above constitute members of at least two genera. While the definition and delineation of genera is scientifically less than clear [84-86], to avoid confusion, the uncharacterised isolates $\mathrm{NH}_{9}-\mathrm{P}_{7}, \mathrm{E} 8$, and PAMC 27224 and 27225 will only be referred to by their strain identifier from here on, and not assigned to a genus or species. When, throughout the thesis, individual species names are mentioned, these will always refer to the type strains described above, as defined in their original publications (refs. $[76,78,79]$ ).

In consequence, it is also wrong to speak of this group as the $\mathrm{Oc}^{-}$ tadecabacter genus, or the Octadecabacters. Rather, it is more adequate to use the terms group and, if phylogenetically valid, clade. The current recommendation for node clade nomenclature is to assign the name of the earliest-described taxon [87]. For informal, e. g. unpublished, clade designations, the name should not be italicised [87]. Consequently, provided that all of the included comparison strains share one common ancestor, which is at the same time exclusive to them, it would be appropriate to speak of the Octadecabacter clade. In the absence of converse information, the term Octadecabacter clade, or simply Octadecabacters, will be used throughout this thesis to refer to this group of nine strains.

\subsection{RATIONALE}

The majority of habitats on Earth are cold, with the cryosphere covering $\approx 20 \%$ of its surface [88], and cold deep water making up most of the oceans' volume [17]. Still, only few roseobacter isolates are available from these habitats, although this group is abundant there as well [55, $89,90]$. Two such isolates, O. arcticus and O. antarcticus, were recently genomically compared [61], and provided first insights into the genetic equipment of cold-adapted roseobacters, albeit without a proper basis for comparison, due to the unavailability of closely related genome sequences.

Since multiple isolates in close relation to the Octadecabacter genus have recently become available (some of them from polar habitats), 
a more general comparison is possible. It is therefore the aim of this thesis to identify genomic features common to cold-adapted Octadecabacters, using a comparative genomics approach. Comparison with prior analyses should show, in how far mechanisms of cold adaptation known from other organisms are reflected in the polar Octadecabacter genomes. Furthermore, the combined genomic information can provide insights into the source of the polar Octadecabacters' adaptability, which they require to colonise their extreme habitat. As the number of available roseobacter genome sequences has risen steadily in recent years, the general and polar-specific genomic trends observed in Octadecabacters will also be related to other Roseobacter group genera and some of their few polar isolates currently available. Notably, comparative analyses within Roseobacter group genera concerning adaptation to extreme environments are generally lacking. Therefore, the present thesis is also intended as a reference for future analyses, as more genomic information will no doubt become available over the next years.

This thesis exclusively follows a comparative genomics approach. In a first step, the genomes of four recently isolated, Octadecabacterassociated strains (introduced in Section 1.4) will be sequenced using NGS methods, availing a total of nine genomes for comparative analysis. This helps to establish a reliable phylogeny of these genomes in the larger context of the Roseobacter group using phylogenomic methods. These methods are better suited than polyphasic studies or comparisons of the $16 \mathrm{~S}$ rRNA gene for phylogenetic analyses, since they incorporate all of the genomic information available for an organism [91]. Sub-clades in the phylogeny, as well as clusters of increased pairwise sequence similarity will then serve as the basis for gene content comparisons: on the one hand, genome annotation and subsequent orthologue detection are used to determine and interpret orthologous groups (OGs) specific to individual Octadecabacter sub-groups. These observations are complemented with a more general comparison of functional gene content, as captured by the cluster of orthologous groups (COG) classification.

A robust phylogeny furthermore enables to infer changes of e. g. gene content, or sequence characters along ancestral lineages. Phylogenetic birth-and-death models provide a powerful method to that end [92], and were, for example, used to analyse the divergence of life strategies between roseobacters and the closely related SAR 11 lineage [93]. In short, such a model assumes that changes of gene family sizes along the edges of a phylogenetic tree result from a stochastic process, which is characterised by three parameters, $k, \lambda$, and $\mu$. Loss within a family of size $n$ occurs at a rate of $n \times \mu$ and gain at $k+\frac{n}{\lambda}$. All three parameters possess a node-specific and a gene family-specific component, and thus may vary between nodes and families. Given a phylogeny and a corresponding phyletic pattern, the parameters are first optimised on the data in a maximum likelihood (ML) setting. In a second step, the 
parameter values can be used to estimate probabilities of gene family changes at the deeper nodes of the phylogeny. This thesis aims to associate specific changes in functional gene content with the colonisation of polar habitats by using a phylogenetic birth-and-death model.

It is important to note that gene content analyses based purely on sequence data have a significant disadvantage: what we can find is always limited by our prior knowledge. With a large number of genomes, one has to rely on automatic annotation to infer gene function, a process which itself can rely on erroneous data (see ref. [94] for one example). Furthermore, research has mostly focused on pathways of the core-metabolism in the past, but these represent only one facet of the cell's biochemical network. As a consequence, we lack a deeper understanding of pathways involved in, e.g. metabolite repair and quality control [95], and a lot of essential genes still possess an unknown, or at best speculative, function [96]. This bias in our current knowledge will inevitably influence the way in which the results of this thesis are interpreted and discussed. While an effort will be made to point out uncertainties and provide alternative explanations where appropriate, it is beyond the scope of this thesis to experimentally test the role and involvement of every component found to differ between polar and temperate strains. Rather, the conclusions drawn here can be the starting point of further experimental analyses concerning the ecology of this exceptional group of organisms.

Finally, beyond the coordinates from which individual Octadecabacters and associated strains were isolated, no investigation into their global distribution and abundance has yet been undertaken. With a multitude of metagenome sequences from a range of sources currently available, a further aim of this thesis is to test and compare the occurrence of sequences related to specific Octadecabacters throughout different habitats and geographic locations. 



\subsection{GENOME SEQUENCING}

Genomes of four strains associated with the Octadecabacter genus were sequenced in this study, as noted in Table 2.2. All strains were cultivated in marine broth (MB) medium (Table 2.1) at a temperature of $8^{\circ} \mathrm{C}$ (isolates PAMC 27224 and 27225 ), or $20^{\circ} \mathrm{C}$ (O. ascidiaceicola and $P$. jejudonensis). Cells were harvested by centrifuging, and their DNA extracted using the MasterPure ${ }^{\mathrm{TM}}$ DNA Purification Kit (Epicentre, Madison, WI, USA), adhering to the manufacturer's instructions. Genomic shotgun paired-end libraries were prepared and sequenced on an Illumina MiSeq (Illumina, San Diego, CA, USA), using the MiSeq reagent kit version 3 according to the manufacturer's instructions.

Table 2.1: Marine broth medium composition. Amounts given refer to one litre of medium. Adjust $\mathrm{pH}$ to 7.6 using $\mathrm{HCl}$ and $\mathrm{NaOH}$, and sterilise the medium via autoclaving.

\begin{tabular}{rcc}
\hline COMPONENT & A MOUNT \\
\hline Bacto peptone & 5.0 & $\mathrm{~g}$ \\
Bacto yeast extract & 1.0 & $\mathrm{~g}$ \\
$\mathrm{Fe}(\mathrm{III})$ citrate & 0.1 & $\mathrm{~g}$ \\
$\mathrm{NaCl}$ & 19.45 & $\mathrm{~g}$ \\
$\mathrm{MgCl}_{2}$ (anhydrous) & 5.9 & $\mathrm{~g}$ \\
$\mathrm{Na}_{2} \mathrm{SO}_{4}$ & 3.24 & $\mathrm{~g}$ \\
$\mathrm{CaCl}_{2}$ & 1.8 & $\mathrm{~g}$ \\
$\mathrm{KCl}$ & 0.55 & $\mathrm{~g}$ \\
$\mathrm{NaHCO}_{3}$ & 0.16 & $\mathrm{~g}$ \\
$\mathrm{KBr}^{2}$ & 80 & $\mathrm{mg}$ \\
$\mathrm{SrCl}_{2}$ & 34 & $\mathrm{mg}$ \\
$\mathrm{H}_{3} \mathrm{BO}_{3}$ & 22 & $\mathrm{mg}$ \\
sodium silicate & 4 & $\mathrm{mg}$ \\
$\mathrm{NaF}^{2}$ & 2.4 & $\mathrm{mg}$ \\
$\left(\mathrm{NH}_{4}\right) \mathrm{NO}_{3}$ & 1.6 & $\mathrm{mg}$ \\
$\mathrm{Na}_{2} \mathrm{HPO}_{4}$ & 8 & $\mathrm{mg}$ \\
\hline $\mathrm{ddH}_{2} \mathrm{O}$ & $\mathrm{ad} 1, \mathrm{oOO} \mathrm{ml}$ \\
\hline
\end{tabular}




\subsection{GENOME ASSEMbly, ANNOTAtion, AND Statistics}

The sequencing reads obtained from Section 2.1 were quality-filtered using Trimmomatic, version 0.32 [97], and subsequently assembled using the SPAdes genome assembler, version 3.5.0 [98]. All contigs $>500$ bp (O. ascidiaceicola), or $\geqslant 2,500$ bp (P. jejudonensis, isolates PAMC 27224 and 27225) were annotated using Prokka [99].

Basic genome statistics were summarised through the use of genomes_ feature_table.pl [100] with the option -p to include plasmids in the calculations. Completeness was assessed using CheckM [101], which scanned each genome for the presence of 528 marker genes from the Rhodobacteraceae family. To assist with examining the presence or absence of specific functions, Kyoto Encyclopedia of Genes and Genomes (KEGG) pathways were reconstructed in all nine genomes [102]. The annotation of KEGG orthology (KO) terms, as well as the pathway mapping were performed via KEGG's web-interface ${ }^{1}$.

\subsection{SEQUENCE COMPARISON OF OCTA DECABACTER CLADE GENOMES}

To clearly delineate species and strain relationships among the Octadecabacters, their genome sequences were subjected to pairwise digital DNA-DNA hybridisation (DDH) [103]. All possible pairings of the nine genomes were submitted to the genome-to-genome distance calculator (GGDC), version 2.1, through the web interface ${ }^{2}$ provided by the Deutsche Sammlung von Mikroorganismen und Zellkulturen (DSMZ). The recommended formula $d_{4}$ [103] and its derived distances were used for downstream analyses.

\subsection{BACTERIAL GENOMES USED FOR COMPARATIVE ANALYSIS}

A wide range of available genome sequences from members of the Roseobacter group were used to create a multilocus sequence analysis (MLSA) phylogeny (Section 2.6), and to analyse their pan-genome (Section 2.10.1). Table 2.2 lists the respective strains, as well as their original publication, where available. Relevant metadata, as well as the genome accession number for each strain are listed in Table A.1 on page 125 .

1 https://www .kegg.jp/kegg/mapper.html

2 http://ggdc.dsmz.de/ggdc.php 
Table 2.2: Genomes for comparative analysis. The REFERENCE column lists, in order of preference, the publication of a strain's isolation and characterisation, the genome data report, the accession number, or $n$. a. if none of the former are available; data sources were either GenBank (GB), the Integrated Microbial Genomes (IMG) database, or this study, as indicated in the sourcE column.

\begin{tabular}{|c|c|c|}
\hline STRAIN & SOURCE & REFERENCE \\
\hline $\begin{array}{l}\text { Aleiiroseovarius crassostreae } \\
\text { CV } 919-312 \mathrm{Sm}\end{array}$ & GB & $\begin{array}{l}\text { Boettcher, Barber, } \\
\text { and } \\
\text { Singer } 1999 \text { [104] }\end{array}$ \\
\hline Celeribacter baekdonensis $\mathrm{B}_{30}$ & GB & AMRK_oooooooo \\
\hline Celeribacter indicus $\mathrm{P}_{73}$ & GB & Lai et al. 2014 [105] \\
\hline Citreicella aestuarii 357 & GB & $\begin{array}{l}\text { Suarez-Suarez } \\
\text { et al. } 2012 \text { [106] }\end{array}$ \\
\hline Citreicella sp. SE45 & GB & ACNW_oooooooo \\
\hline $\begin{array}{l}\text { Rhodobacterales bacterium } \\
\text { HTCC } 2255\end{array}$ & GB & NZ_AATR_oooooooo \\
\hline Phaeobacter sp. LSS9 & IMG & n. a. \\
\hline $\begin{array}{l}\text { Dinoroseobacter shibae } \\
\text { DSM } 16493\end{array}$ & GB & Biebl et al. 2005 [107] \\
\hline $\begin{array}{l}\text { Jannaschia aquimarina } \\
\text { GSW-M26 }\end{array}$ & GB & $\begin{array}{l}\text { Park and } \\
\text { Yoon } 2012[108]\end{array}$ \\
\hline Jannaschia rubra DSM 16279 & IMG & $\begin{array}{l}\text { Macián } \\
\text { et al. 2005 [109] }\end{array}$ \\
\hline Jannaschia sp. CCS1 & GB & $\begin{array}{l}\text { Moran } \\
\text { et al. } 2007[70]\end{array}$ \\
\hline $\begin{array}{l}\text { Ketogulonicigenium vulgare } \\
\text { WSH-oo1 }\end{array}$ & GB & Liu et al. $2011[110]$ \\
\hline Ketogulonicigenium vulgare $\mathrm{Y}_{25}$ & GB & $\begin{array}{l}\text { Xiong } \\
\text { et al. } 2011[111]\end{array}$ \\
\hline $\begin{array}{l}\text { Leisingera aquaemixtae } \\
\text { CECT } 8399\end{array}$ & GB & Park et al. 2017 [112] \\
\hline $\begin{array}{l}\text { Leisingera aquimarina } \\
\text { DSM } 24565\end{array}$ & GB & $\begin{array}{l}\text { Vandecandelaere } \\
\text { et al. } 2008 \text { [113] }\end{array}$ \\
\hline Leisingera caerulea DSM 24564 & GB & $\begin{array}{l}\text { Vandecandelaere } \\
\text { et al. 2009 [114] }\end{array}$ \\
\hline $\begin{array}{l}\text { Leisingera daeponensis } \\
\text { DSM } 23529\end{array}$ & GB & Yoon et al. 2007 [115] \\
\hline $\begin{array}{l}\text { Leisingera methylohalidivorans } \\
\text { DSM } 14336\end{array}$ & GB & $\begin{array}{l}\text { Schaefer } \\
\text { et al. } 2002 \text { [116] }\end{array}$ \\
\hline Leisingera sp. ANG1 & GB & $\begin{array}{l}\text { Collins and } \\
\text { Nyholm } 2011[117]\end{array}$ \\
\hline
\end{tabular}


Table 2.2: continued

\begin{tabular}{|c|c|c|}
\hline STRAIN & SOURCE & REFERENCE \\
\hline Litoreibacter albidus DSM 26922 & GB & $\begin{array}{l}\text { Romanenko } \\
\text { et al. } 2011[118]\end{array}$ \\
\hline Litoreibacter arenae DSM 19593 & GB & Kim et al. 2009 [119] \\
\hline $\begin{array}{l}\text { Litoreibacter ascidiaceicola } \\
\text { DSM } 100566\end{array}$ & GB & Kim et al. 2016 [120] \\
\hline $\begin{array}{l}\text { Litoreibacter janthinus } \\
\text { DSM } 26921\end{array}$ & GB & $\begin{array}{l}\text { Romanenko } \\
\text { et al. } 2011[118]\end{array}$ \\
\hline Loktanella atrilutea DSM 29326 & GB & $\begin{array}{l}\text { Hosoya and } \\
\text { Yokota } 2007[121]\end{array}$ \\
\hline Loktanella cinnabarina LL-o01 & GB & $\begin{array}{l}\text { Tsubouchi } \\
\text { et al. 2013 [122] }\end{array}$ \\
\hline $\begin{array}{l}\text { Loktanella fryxellensis } \\
\text { DSM } 16213\end{array}$ & GB & $\begin{array}{l}\text { VanTrappen, } \\
\text { Mergaert, and } \\
\text { Swings } 2004 \text { [55] }\end{array}$ \\
\hline $\begin{array}{l}\text { Loktanella hongkongensis } \\
\text { DSM } 17492\end{array}$ & GB & Lau et al. 2004 [123] \\
\hline Loktanella koreensis DSM 17925 & GB & $\begin{array}{l}\text { Weon } \\
\text { et al. } 2006 \text { [124] }\end{array}$ \\
\hline Loktanella litorea DSM 29433 & GB & $\begin{array}{l}\text { Yoon, Jung, and } \\
\text { Lee } 2013 \text { [125] }\end{array}$ \\
\hline $\begin{array}{l}\text { Loktanella pyoseonensis } \\
\text { DSM } 21424\end{array}$ & GB & $\begin{array}{l}\text { Moon } \\
\text { et al. 2010 [126] }\end{array}$ \\
\hline Loktanella rosea DSM 29591 & GB & $\begin{array}{l}\text { Ivanova } \\
\text { et al. } 2005[127]\end{array}$ \\
\hline Loktanella salsilacus DSM 16199 & GB & $\begin{array}{l}\text { VanTrappen, } \\
\text { Mergaert, and } \\
\text { Swings } 2004 \text { [55] }\end{array}$ \\
\hline $\begin{array}{l}\text { Loktanella sediminum } \\
\text { DSM } 28715\end{array}$ & GB & $\begin{array}{l}\text { Liang } \\
\text { et al. 2015 [128] }\end{array}$ \\
\hline Loktanella sp. SE62 & IMG & n. a. \\
\hline Loktanella tamlensis DSM 26879 & GB & Lee 2012 [129] \\
\hline $\begin{array}{l}\text { Loktanella vestfoldensis } \\
\text { DSM } 16212\end{array}$ & GB & $\begin{array}{l}\text { VanTrappen, } \\
\text { Mergaert, and } \\
\text { Swings } 2004 \text { [55] }\end{array}$ \\
\hline Loktanella vestfoldensis SKA53 & GB & NZ_AAMS_00000000 \\
\hline Marinovum algicola DG 898 & GB & $\begin{array}{l}\text { Green } \\
\text { et al. } 2004 \text { [130] }\end{array}$ \\
\hline
\end{tabular}


Table 2.2: continued

\begin{tabular}{|c|c|c|}
\hline STRAIN & SOURCE & REFERENCE \\
\hline $\begin{array}{l}\text { Maritimibacter alkaliphilus } \\
\text { HTCC } 2654\end{array}$ & GB & Lee et al. 2007 [131] \\
\hline Maritimibacter sp. HL-12 & GB & NZ_FXBQ_oooooooo \\
\hline Nautella italica DSM 26436 & GB & $\begin{array}{l}\text { Vandecandelaere } \\
\text { et al. } 2009 \text { [132] }\end{array}$ \\
\hline Nautella italica R11 & GB & NZ_ABXM_oooooooo \\
\hline Nereida ignava CECT 5292 & GB & $\begin{array}{l}\text { Pujalte } \\
\text { et al. } 2005 \text { [133] }\end{array}$ \\
\hline Oceanibulbus indolifex HEL-45 & GB & n. a. \\
\hline Oceanicola batsensis HTCC 2597 & GB & $\begin{array}{l}\text { Cho and Giovan- } \\
\text { noni } 2004[134]\end{array}$ \\
\hline $\begin{array}{l}\text { Oceanicola granulosus } \\
\text { HTCC } 2516\end{array}$ & GB & $\begin{array}{l}\text { Cho and Giovan- } \\
\text { noni } 2004[134]\end{array}$ \\
\hline $\begin{array}{l}\text { Oceanicola nanhaiensis } \\
\text { DSM } 18065\end{array}$ & GB & Gu et al. 2007 [135] \\
\hline Oceanicola sp. HL-35 & GB & NZ_JAFT_oooooooo \\
\hline Oceanicola sp. MCTG156(1a) & GB & $\begin{array}{l}\text { Gutierrez } \\
\text { et al. } 2017 \text { [136] }\end{array}$ \\
\hline Oceanicola sp. S124 & GB & $\begin{array}{l}\text { Kwon } \\
\text { et al. } 2012 \text { [137] }\end{array}$ \\
\hline $\begin{array}{l}\text { Oceaniovalibus guishaninsula } \\
\text { JLT2003 }\end{array}$ & GB & Liu et al. 2012 [138] \\
\hline Octadecabacter antarcticus 307 & GB & $\begin{array}{l}\text { Gosink, Herwig, and } \\
\text { Staley } 1997[76]\end{array}$ \\
\hline $\begin{array}{l}\text { Octadecabacter arcticus } \\
\text { DSM } 13978\end{array}$ & GB & $\begin{array}{l}\text { Gosink, Herwig, and } \\
\text { Staley } 1997[76]\end{array}$ \\
\hline $\begin{array}{l}\text { Octadecabacter ascidiaceicola } \\
\text { CECT } 8868\end{array}$ & THIS STUDY & Kim et al. 2016 [81] \\
\hline Octadecabacter sp. E8 & IMG & n. a. \\
\hline Octadecabacter sp. $\mathrm{NH}_{9}-\mathrm{P}_{7}$ & IMG & n. a. \\
\hline Octadecabacter temperatus SB1 & GB & $\begin{array}{l}\text { Billerbeck } \\
\text { et al. } 2015 \text { [79] }\end{array}$ \\
\hline Octadecabacter sp. PAMC 27224 & THIS STUDY & Lee et al. 2014 [8o] \\
\hline Octadecabacter sp. PAMC 27225 & THIS STUDY & Lee et al. 2014 [8o] \\
\hline $\begin{array}{l}\text { Parvularcula bermudensis } \\
\text { HTCC } 2503\end{array}$ & GB & $\begin{array}{l}\text { Cho and Giovan- } \\
\text { noni 2003 [139] }\end{array}$ \\
\hline $\begin{array}{l}\text { Pelagibaca bermudensis } \\
\text { HTCC } 2601\end{array}$ & GB & $\begin{array}{l}\text { Cho and Giovan- } \\
\text { noni } 2006 \text { [140] }\end{array}$ \\
\hline
\end{tabular}


Table 2.2: continued

\begin{tabular}{|c|c|c|}
\hline STRAIN & SOURCE & REFERENCE \\
\hline Phaeobacter gallaeciensis BS107 & GB & $\begin{array}{l}\text { Ruiz-Ponte } \\
\text { et al. } 1998[141]\end{array}$ \\
\hline $\begin{array}{l}\text { Phaeobacter gallaeciensis } \\
\text { DSM } 26640\end{array}$ & GB & $\begin{array}{l}\text { Ruiz-Ponte } \\
\text { et al. } 1998 \text { [141] }\end{array}$ \\
\hline $\begin{array}{l}\text { Phaeobacter inhibens } \\
\text { DSM } 16374\end{array}$ & GB & $\begin{array}{l}\text { Martens } \\
\text { et al. } 2006 \text { [142] }\end{array}$ \\
\hline $\begin{array}{l}\text { Phaeobacter inhibens } \\
\text { DSM } 17395\end{array}$ & GB & $\begin{array}{l}\text { Buddruhs } \\
\text { et al. } 2013 \text { [143] }\end{array}$ \\
\hline $\begin{array}{l}\text { Planktomarina temperata } \\
\text { RCA } 23\end{array}$ & GB & $\begin{array}{l}\text { Giebel } \\
\text { et al. } 2013[144]\end{array}$ \\
\hline $\begin{array}{l}\text { Pseudophaeobacter arcticus } \\
\text { DSM } 23566\end{array}$ & GB & $\begin{array}{l}\text { Zhang } \\
\text { et al. } 2008 \text { [56] }\end{array}$ \\
\hline $\begin{array}{l}\text { Pseudooctadecabacter } \\
\text { jejudonensis CECT } 8397\end{array}$ & THIS STUDY & $\begin{array}{l}\text { Park and } \\
\text { Yoon 2014 [78] }\end{array}$ \\
\hline $\begin{array}{l}\text { Rhodobacteraceae bacterium } \\
\mathrm{SB}_{2}\end{array}$ & GB & NZ_LGRT_oooooooo \\
\hline $\begin{array}{l}\text { Rhodobacteraceae bacterium } \\
\text { HTCC } 2083\end{array}$ & GB & Kang et al. 2011 [145] \\
\hline $\begin{array}{l}\text { Rhodobacteraceae bacterium } \\
\text { HTCC } 215^{\circ}\end{array}$ & GB & Kang et al. 2010 [146] \\
\hline Rhodobacterales bacterium $\mathrm{Y}_{4} \mathrm{I}$ & GB & NZ_ABXF_oooooooo \\
\hline $\begin{array}{l}\text { Roseobacter denitrificans } \\
\text { Och } 114\end{array}$ & GB & Shiba 1991 [57] \\
\hline Roseobacter litoralis Och 149 & GB & Shiba 1991 [57] \\
\hline Roseobacter sp. AzwK-3b & GB & $\begin{array}{l}\text { Hansel and } \\
\text { Francis } 2006[147]\end{array}$ \\
\hline Roseobacter sp. CCS2 & GB & NZ_AAYB_00000000 \\
\hline Roseobacter sp. GAI101 & GB & NZ_ABXS_oooooooo \\
\hline Roseobacter sp. LE17 & IMG & n. a. \\
\hline Roseobacter sp. MED193 & GB & NZ_AANB_oooooooo \\
\hline Roseobacter sp. R2A57 & IMG & n. a. \\
\hline Roseobacter sp. SK209-2-6 & GB & NZ_AAYC_oooooooo \\
\hline Roseovarius atlanticus $\mathrm{R}_{12} \mathrm{~B}$ & GB & Li et al. 2016 [148] \\
\hline $\begin{array}{l}\text { Roseovarius mucosus } \\
\text { DSM } 17069\end{array}$ & GB & Biebl et al. 2005 [149] \\
\hline Roseovarius nubinhibens ISM & GB & $\begin{array}{l}\text { González } \\
\text { et al. } 2003 \text { [150] }\end{array}$ \\
\hline
\end{tabular}


Table 2.2: continued

\begin{tabular}{|c|c|c|}
\hline STRAIN & SOURCE & REFERENCE \\
\hline Roseovarius sp. 217 & GB & $\begin{array}{l}\text { Schäfer } \\
\text { et al. 2005 [151] }\end{array}$ \\
\hline Roseovarius sp. MCTG156(2b) & GB & $\begin{array}{l}\text { Gutierrez } \\
\text { et al. } 2017 \text { [152] }\end{array}$ \\
\hline Roseovarius sp. TM1035 & GB & NZ_ABCL_oooooooo \\
\hline Ruegeria atlantica DSM 5823 & GB & $\begin{array}{l}\text { Rüger and } \\
\text { Höfle } 1992 \text { [153] }\end{array}$ \\
\hline Ruegeria conchae TW15 & GB & Lee et al. 2012 [154] \\
\hline $\begin{array}{l}\text { Ruegeria halocynthiae } \\
\text { MOLA R1/13b }\end{array}$ & GB & $\begin{array}{l}\text { Doberva } \\
\text { et al. } 2014 \text { [155] }\end{array}$ \\
\hline $\begin{array}{l}\text { Ruegeria lacuscaerulensis } \\
\text { ITI-1157 }\end{array}$ & GB & NZ_ACNX_0ooooooo \\
\hline Ruegeria mobilis F1926 & GB & $\begin{array}{l}\text { Sonnenschein } \\
\text { et al. } 2017 \text { [156] }\end{array}$ \\
\hline Ruegeria pomeroyi DSS-3 & GB & $\begin{array}{l}\text { González } \\
\text { et al. } 2003 \text { [150] }\end{array}$ \\
\hline Ruegeria sp. TM1040 & GB & NZ_AAFG_oooooooo \\
\hline Sagittula stellata E-37 & GB & $\begin{array}{l}\text { Gonzalez } \\
\text { et al. } 1997 \text { [157] }\end{array}$ \\
\hline $\begin{array}{l}\text { Sedimentalea nanhaiensis } \\
\text { DSM } 24252\end{array}$ & GB & Sun et al. 2010 [158] \\
\hline Shimia marina CECT 7688 & GB & $\begin{array}{l}\text { Choi and } \\
\text { Cho 2006 [159] }\end{array}$ \\
\hline Shimia sp. SKo13 & GB & $\begin{array}{l}\text { Kanukollu } \\
\text { et al. } 2016 \text { [16o] }\end{array}$ \\
\hline $\begin{array}{l}\text { Sulfitobacter donghicola } \\
\text { DSW-25 }\end{array}$ & GB & Yoon et al. 2007 [161] \\
\hline Sulfitobacter geojensis MM-124 & GB & $\begin{array}{l}\text { Kwak } \\
\text { et al. } 2014 \text { [162] }\end{array}$ \\
\hline $\begin{array}{l}\text { Sulfitobacter guttiformis } \\
\text { KCTC } 32187\end{array}$ & GB & $\begin{array}{l}\text { Labrenz } \\
\text { et al. } 2000 \text { [163] }\end{array}$ \\
\hline $\begin{array}{l}\text { Sulfitobacter mediterraneus } \\
\text { KCTC } 32188\end{array}$ & GB & $\begin{array}{l}\text { Pukall } \\
\text { et al. } 1999 \text { [164] }\end{array}$ \\
\hline Sulfitobacter noctilucae NB-68 & GB & $\begin{array}{l}\text { Kwak } \\
\text { et al. } 2014 \text { [162] }\end{array}$ \\
\hline Sulfitobacter noctilucicola NB-77 & GB & $\begin{array}{l}\text { Kwak } \\
\text { et al. } 2014 \text { [162] }\end{array}$ \\
\hline
\end{tabular}


Table 2.2: continued

\begin{tabular}{|c|c|c|}
\hline STRAIN & SOURCE & REFERENCE \\
\hline $\begin{array}{l}\text { Sulfitobacter pontiacus } \\
\text { 3SOLIMARo9 }\end{array}$ & GB & $\begin{array}{l}\text { Mas-lladó } \\
\text { et al. } 2014 \text { [165] }\end{array}$ \\
\hline $\begin{array}{l}\text { Sulfitobacter pseudonitzschiae } \\
\mathrm{H}_{3}\end{array}$ & GB & $\begin{array}{l}\text { Hong } \\
\text { et al. } 2015 \text { [166] }\end{array}$ \\
\hline $\begin{array}{l}\text { Sulfitobacter sp. } \\
\text { 20_GPM-1509m }\end{array}$ & GB & NZ_JIBC_oooooooo \\
\hline Sulfitobacter sp. CB2047 & GB & $\begin{array}{l}\text { Ankrah } \\
\text { et al. } 2014[167]\end{array}$ \\
\hline Sulfitobacter sp. NAS-14.1 & GB & NZ_AALZ_oooooooo \\
\hline Sulfitobacter sp. SA11 & IMG & n. a. \\
\hline Tateyamaria sp. ANG1-S1 & GB & $\begin{array}{l}\text { Collins } \\
\text { et al. } 2015 \text { [168] }\end{array}$ \\
\hline Thalassobium sp. R2A62 & GB & NZ_ACOA_oooooooo \\
\hline $\begin{array}{l}\text { Thalassobacter stenotrophicus } \\
\text { CECT } 5294\end{array}$ & GB & $\begin{array}{l}\text { Macián } \\
\text { et al. 2005 [169] }\end{array}$ \\
\hline Wenxinia marina DSM 24838 & GB & Ying et al. 2007 [170] \\
\hline
\end{tabular}

\subsection{ORTHOLOGUE DETECTION}

The amino acid sequences of all coding DNA sequences (CDSs) were extracted from the genomes listed in Table 2.2 using cds_extractor.pl, version 0.7 [100], and written to multifasta files. Proteinortho5 [171] was used with the options -synteny, -selfblast and -singles to determine orthologous and paralogous relationships among the extracted proteins. Minimum identity and coverage values for basic local alignment search tool (BLAST) hits to be reported were set to $35 \%$ and $65 \%$, respectively.

\subsection{ROSEOBACTER GROUP PHYLOGENY RECONSTRUCTION}

A MLSA phylogeny was reconstructed from the strict, non-redundant

core-genome-genes common to all members of a group of organisms (i. e. excluding genes with paralogues) core-genome, of all Roseobacter group members listed in Table 2.2. The respective orthologous protein sequences were aligned with the multiple sequence comparison by log-expectation (MUSCLE) algorithm via its homonymous program, version 3.8.31 [172], and the alignments were concatenated to a supermatrix. Positions containing gaps were removed, resulting in a final alignment length of 23,052 patterns (139 genes). Using the PTHREADS implementation of RAxML (version 8.1.22) [173], the roseobacter phylogeny was reconstructed in a ML-framework under the WAG model of 
amino acid substitution (with ML-optimised base frequencies and substitution rates) and a gamma distribution to model rate heterogeneity (discretised into four categories, $\alpha$ parameter estimated through ML). Five hundred bootstraps were computed on random maximum parsimony (MP) starting trees to estimate branch support. Furthermore, ML phylogenies were calculated for each of the 139 core-genes individually, using the same configurations given above. These were then used to estimate bipartition support in the concatenation-based phylogeny through calculation of internode certainty (IC) and tree certainty (TC) scores [174]. In short, bipartitions of the super-matrix-derived phylogeny were evaluated for their frequencies in the 139 individual trees and related to the two most frequent conflicting bipartitions. IC and TC scores reflect this relation, with values close to o indicating that multiple conflicting bifurcations occur at the same frequency, and values close to 1 indicating the absence of such conflicts [174]. The scores were calculated using the respective option of RAxML.

In addition, a hierarchical clustering was computed for all compared genomes, based on the pairwise Jaccard distances in their gene content. The distances were derived from the orthology information (including singletons), and binarised into presence or absence of individual OGs using the scikit-learn package for python [175]. Hierarchical clustering was performed using the Farthest Point Algorithm implementation of scipy [176] and the clusters were visualised in form of a tree. Two trees were calculated this way, one using the distances derived from all OGs, and one for which core OGs of deeper-branching clades in the phylogeny were marked as absent in all the clade's corresponding members prior to distance calculation. The second tree therefore reflects the similarity of the cloud-genomes of different clades in the phylogeny, as well as the shell-genomes of their individual members. The amount of differing bifurcations between both these trees and the reconstructed phylogeny was expressed as the Robinson-Foulds (RF) distance, and calculated using the Environment for (phylogenetic) Tree Exploration (ete3) package for python [177].

\subsection{OCTADECABACTER CLADE PHYLOGENY AND GENE CONTENT CLUSTERING}

Individual phylogenies of all 1,513 Octadecabacter core-genes without paralogues were reconstructed in RAxML, as described in Section 2.6, with the difference that no bootstrap analyses were performed. The resulting ML trees were combined into a cluster consensus network using Dendroscope, version 3.5.9 [178]. Only splits occurring in at least $20 \%$ of trees were visualised. Support of the network's bipartitions was estimated via their frequencies in the 1,513 individual trees and expressed in form of IC and TC scores [174], calculated by RAxML (see Section 2.6). In order to compute the scores, two trees were compared

cloud-genomegenes present in only a subset of all compared genomes

shell-genome-genes present in one or few of the compared genomes 
against the rest (reflecting ambiguous positioning of $O$. antarcticus), to ascertain consistency of the calculated scores.

Clustering the Octadecabacters according to their gene content was based on pairwise Jaccard distances, and performed as described in Section 2.6.

\subsection{COG ANNOTATION}

The COG annotation followed the IMG standard operating procedure (SOP) workflow [179] with a few exceptions. Pre-computed positionspecific scoring matrices (PSSMs) of all COGs were acquired from the National Center for Biotechnology Information (NCBI)'s Conserved Domains Database $(\mathrm{CDD})^{3}$. These were used as reference in reverse position-specific BLAST (rpsBLAST) runs with the genomes' protein multifasta files (created in Section 2.5) as queries. Only the best BLAST hit for each protein was reported, with an e-value cutoff of $10^{-5}$. Tabseparated output was forced using BLAST's -outfmt 6 option. The script cdd2 cog.pl version 0.1 [100] parsed the resulting tables and assigned BLAST hits to the appropriate COGs, using the COG descriptions and associations defined in the most recent update from 2014 [180].

\subsection{BIRTH-AND-DEATH MODEL OF FUNCTIONAL GENE CONTENT EVOLUTION}

A general phylogenetic birth-and-death model of family size evolution was optimised on the COG data, as implemented in the program Count [181]. Owing to the model complexity and a lack of parallelisation, optimisation was only computationally feasible for a subset of the 116 strains included in the reference phylogeny. Therefore, a sub-tree of 32 taxa was pruned from the MLSA phylogeny created in Section 2.6, comprising mostly the Octadecabacter and Loktanella clades, more precisely all organisms contained in the equivalents to clades 3, 4, and 5, which Simon et al. recently defined [59]. COG family numbers for each of the 32 genomes were compiled to a phyletic pattern and provided to CounT, together with the reference phylogeny. Multiple runs of parameter estimation were performed with successively increasing model complexities, starting from uniformity of parameters across tree edges and gene families, and ending with gamma-distributed parameter values, discretised into two to three categories. Complexity of the model was only further increased if the increase in likelihood justified the higher number of free parameters. The final model contained two rate categories for gene loss, and three categories each for duplication, transfer, and length. Using the estimated parameter values, probabilities of gain, loss, expansion, and reduction for each COG at each tree node were calculated by CounT. For downstream analysis, only those

3 ftp.ncbi.nih.gov/pub/COG/COG2014/data/ 
COGs were considered, which showed a probability of change $>0.5$ at the particular node in question.

\subsection{GENE CONTENT ANALYSES}

\subsubsection{Pan- and core-genome calculations}

The pan-genome and strict core-genome sizes of the Octadecabacters were both derived from the orthology information obtained in Section 2.5. Their dependence on the number of included genomes was determined by calculating both for each possible combination of one to nine Octadecabacter genomes (without replacement) and subsequent least-squares fit. A power law (Equation (2.1)) and exponential decay (Equation (2.2)) function were fitted, respectively, to the median values of pan- and core-genome size using the scipy module for the python programming language [176]. In an equivalent analysis for the whole Roseobacter group (genomes listed in Table 2.2, excluding the outgroup genome Parvularcula bermudensis and Phaeobacter sp. LSS9), approximately 600 combinations were drawn at random for each number of species included, as testing all possible combinations was not computationally feasible.

$$
\begin{aligned}
& f(x)=a \cdot x^{b}+c \\
& f(x)=a \cdot e^{b \cdot x}+c
\end{aligned}
$$

\subsubsection{Determination of group-specific orthologous groups}

The Octadecabacter clade genomes were divided into four groups, according to the clusters based on their pairwise distances in gene content (Section 2.7). Group I contained the polar species O. arcticus, $O$. antarcticus, and isolates PAMC 27224 and 27225 , group II the temperate species $O$. temperatus and $O$. ascidiaceicola, group III $P$. jejudonensis and isolate $\mathrm{NH}_{9}-\mathrm{P}_{7}$ and group IV the single genome of isolate E8. Together with the orthology data, the script po2group_stats.pl, version 0.1.3 [100] determined which OGs were specific to each group, i. e. which genes occurred in all members of a group and none of the other compared genomes.

\subsection{PROTEIN SECONDARY STRUCTURE PREDICTION AND COM- PARISON}

The secondary structure content of each annotated protein in the nine Octadecabacter genomes was predicted from the primary amino acid sequence using the algorithm implemented in the JPred4 secondary structure prediction server [182]. JPred4 returns the secondary structure state of each amino acid position in the protein (either helix, sheet, 
Benjamini-Hochberg correction controls the false discovery

rate or none), along with a confidence score. To summarise the total content of $\alpha$-helix and $\beta$-sheet, amino acids, which were assigned to one of the two, were counted fully if their confidence score was at least 7 , and as one-half if it was 5 or 6 . The absolute number of amino acids of each structural feature was divided by the length of the protein to obtain the relative content of both $\alpha$-helix and $\beta$-sheet. Utilising the orthology information (Section 2.5), the nine genomes were hierarchically clustered based on the pairwise euclidean distances in relative secondary structure content of the core-proteome. Clustering was performed as described in Section 2.6.

Statistical difference in secondary structure content was tested separately for helix and sheet through two-sided Wilcoxon signed-rank tests, as implemented in scipy [176]. This tests the null-hypothesis $\mathrm{H}_{0}$ that the median difference between two samples equals zero. For each possible pairing of two Octadecabacter clade genomes, the relative structure content of either helix or sheet in all core proteins served as the observations, based on which the test statistic and the corresponding $p$-value were computed. In order to assert that the median difference between samples $\neq 0$, i.e. to accept $H_{1}$, the significance level $\alpha$ was corrected for multiple statistical testing via the method described by Benjamini and Hochberg [183]. In short, the p-values were sorted in ascending order and assigned ranks. Then, each was compared to its Benjamini-Hochberg critical value given by $\frac{i}{m} \times \alpha$, where $i$ is the value's rank, $m=36$ the total number of performed tests and $\alpha=0.05$ the desired significance level, corresponding to the false discovery rate (FDR). The highest $p$-value, for which $p<\frac{i}{m} \times \alpha$, and all values smaller than it were considered significant in rejecting $\mathrm{H}_{0}$ and indicating that the respective pair of Octadecabacters shows differences in the particular structural feature. The direction of this difference was tested in subsequent one-sided Wilcoxon signed-rank tests, with the hypothesis $\mathrm{H}_{0}$ that the median difference between the samples is either positive, or negative.

General comparisons of amino acid content were carried out for Octadecabacter strains with highly similar GC-contents, which excluded P. jejudonensis and isolate $\mathrm{NH}_{9}-\mathrm{P}_{7}$. In $\chi^{2}$ tests, observed numbers of the twenty amino acids were compared to their expected numbers from the base frequencies (averaged over all seven clade members) for each strain. Mean frequencies of the amino acids in general, as well their ratios of occurrence in $\alpha$-helices versus non-helix regions, were compared between the genome groups defined in Section 2.10.2.

\subsection{OCTADECABACTER OCCURRENCE IN METAGENOMES}

The global distribution of Octadecabacters was studied through metagenomic read mapping. The workflow consisted of three main steps: 
(i) selection of metagenomes, (ii) read mapping, and (iii) filtering the obtained results for unspecific hits and subsequent normalisation.

\subsubsection{Selection of metagenomes}

In order to keep the workflow computationally feasible, only metagenomes available on the Rapid Annotation using Subsystems Technology (RAST) for metagenomes (mg-RAST) platform [184] were considered. In a pre-selection step, identifiers and meta-data of metagenomes, in which Octadecabacters constituted at least $1 \%$ of reads assigned to the $\alpha$-Proteobacteria, were acquired. These metagenomes' reads were then downloaded through the mg-RAST application programming interface (API) and used for subsequent mapping.

\subsubsection{Read mapping}

Due to their high conservation, rRNA gene clusters were excised from the Octadecabacter genomes prior to read mapping. Their positions were identified using rnammer [185] and the sequence between the coordinates excised. The genome DNA sequences were then concatenated into a single file. Bowtie2 [186], version 2.3.1, was used to map the reads. Alignment seed length was set to 12, with at maximum one mismatch allowed per seed. Penalties for mismatches, as well as undefined bases ("N"), were set to 3, gap openings and extensions in both reference and read were penalised with 3 and 5 , respectively. The minimum score $x$ for a hit to be reported had to satisfy $x \geqslant-0.2 \times$ readlength -6 , corresponding to a minimum nucleotide identity of approximately $90 \%$ between read and reference. Only the best hit was reported.

\subsubsection{Filtering and normalisation}

Unspecific hits were filtered using the available Roseobacter group genome information (Table 2.2). To that end, their nucleotide sequences were split into fragments of 1,00o nt length, which were then subjected to pairwise BLAST comparisons between the genomes. Both steps are part of the average nucleotide identity (ANI) comparison workflow of the python package pyANI [187]. Subsequently, reads which mapped with at least $65 \%$ of their length to Octadecabacter regions with $>90 \%$ nucleotide identity to regions in other roseobacters were discarded, and the remaining hits were considered specific to Octadecabacters. The comparison of nucleotide identity included comparisons between Octadecabacter groups, but not within them. This led to the exclusion of less reads, but restricted the analysis to the level of Octadecabacter groups. 
As a means of normalisation, for each metagenome the number of nucleotides in specific hits was divided by the number of nucleotides in reads assigned to the bacteria kingdom by mg-RAST.

In order to differentiate between individual Octadecabacters, the same filtering and normalisation procedure described above was applied once more, but included the pairwise comparisons between all nine Octadecabacter genomes. 


\subsection{GENOME ASSEMBLY AND GENERAL COMPARISON}

General metrics of the analysed Octadecabacter clade genomes are given in Table 3.1. Despite being highly fragmented with $\approx 130$ contigs, the two PAMC isolate genomes are over $99 \%$ complete, based on the presence of 528 Rhodobacteraceae marker genes. Polar Octadecabacters possess, on average, larger genomes than temperate clade members (by $\approx 800 \mathrm{CDSs}$ ), combined with a lower coding percentage. The DNA GC-content is about $55 \%$ in most strains, and markedly higher (about $60 \%$ ) in isolate $\mathrm{NH}_{9}-\mathrm{P}_{7}$ and P. jejudonensis. Based on the orthology data, the Octadecabacter clade's core-genome size is 1,609 (compared to 142 over the whole Roseobacter group, including the outgroup genome). Corresponding NCBI accession numbers are provided in Table A.1, p. 125.

Table 3.1: Basic metrics of Octadecabacter clade genomes. STATUs is listed as either finished (F), or (permanent) draft (D). For finished genomes, grey numbers in parentheses indicate the number of plasmids. Plasmids were included in determining genome size, contig number, and CDS count.

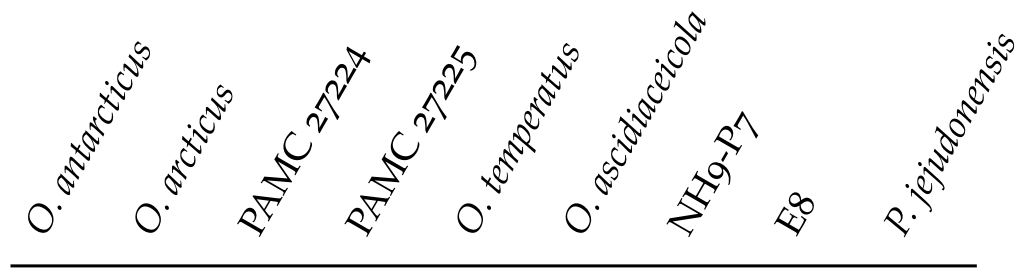

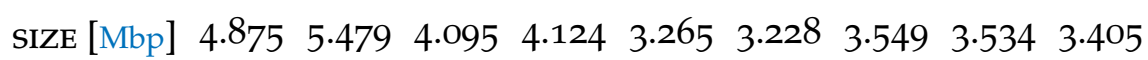

\begin{tabular}{|c|c|c|c|c|c|c|c|c|c|}
\hline STATUS & F & F & D & D & $\mathrm{F}$ & D & D & D & D \\
\hline CONTIGS & $2(1)$ & $3(2)$ & 132 & 129 & $2(1)$ & 11 & 18 & 13 & 20 \\
\hline $\begin{array}{l}\text { OMPLETE- } \\
\text { NESS }\end{array}$ & & & & & $>99 \%$ & & & & \\
\hline GC $\%$ & 54.62 & 55.15 & 55.11 & 55.15 & 54.68 & 54.94 & 59.88 & 54.35 & $59 \cdot 57$ \\
\hline CDSs & 4,569 & 4,694 & 4,188 & 4,161 & 3,294 & 3,283 & 3,484 & 3,484 & 3,345 \\
\hline ODING \% & 80.91 & 78.34 & 88.77 & 88.71 & 91.86 & 91.51 & 91.40 & 90.44 & 91.22 \\
\hline COGs & 3,548 & 3,851 & 3,267 & 3,273 & 2,717 & 2,671 & 2,883 & 2,914 & 2,772 \\
\hline
\end{tabular}

Pairwise genome similarities were determined as digital DNA-DNA hybridisation (DDH) values using the genome-to-genome distance calculator (GGDC) [103] and are visualised in Figure 3.1. Notably, the two 
PAMC isolates show $98 \%$ similarity to each other, but only $\approx 30 \%$ to $O$. antarcticus. The genome of $O$. ascidiaceicola is most similar to $O$. temperatus, albeit at a low DDH value of $\sim 25 \%$, while the two strains E8 and $\mathrm{NH}_{9}-\mathrm{P}_{7}$ are less than $20 \%$ similar to any other clade member analysed (the same is true for Pseudooctadecabacter jejudonensis). Based on the DDH-derived distances, isolate $\mathrm{NH}_{9}-\mathrm{P}_{7}$ and $P$. jejudonensis form a separate cluster, which coincides with their higher GC-content (Table 3.1). The novel polar isolates PAMC 27224 and PAMC 27225 form a distinct and comparatively deep-branching cluster with the type strains of the polar species $O$. antarcticus and O. arcticus, neighbouring a corresponding non-polar cluster formed by $O$. temperatus and $O$. ascidiaceicola.

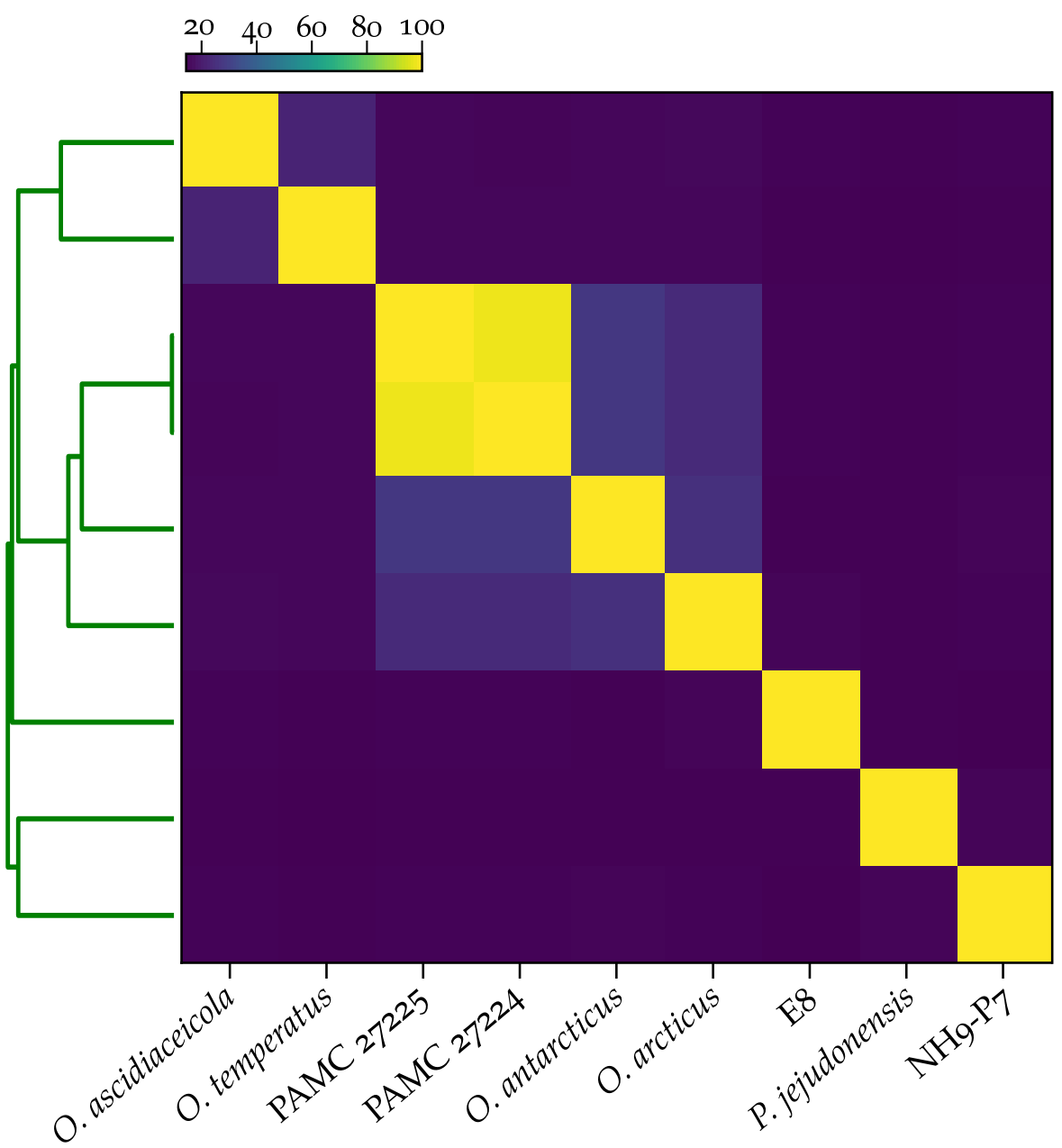

Figure 3.1: Percent similarity values of pairwise Octadecabacter clade genome comparisons via GGDC, presented as a heat-map. Dendrogram on the left represents hierarchical clustering based on the pairwise distances calculated from the DDH values. 


\subsection{PHYLOGENY}

A MLSA-phylogeny based on 139 core-genes of the Roseobacter group (including the outgroup genome Parvularcula bermudensis) generally displays the same topology, which other studies observed $[59,60]$ and clusters defined therein can be reproduced here (marked respectively in Figure A.2, p. 131). It also verifies the newly sequenced strains association with the Octadecabacters, since they form a coherent clade with strong bootstrap support (Figure 3.2). Their inferred phylogenetic relationships also mirror the similarity- (DDH-)based observations (see Figure 3.1). The earliest branching and therefore most distant member is isolate $\mathrm{NH}_{9}-\mathrm{P}_{7}$, followed by $\mathrm{P}$. jejudonensis, although this bifurcation is associated with some uncertainty (low bootstrap support value of 64). All polar isolates cluster together and form a common clade with O. temperatus and O. ascidiaceicola, which means that they share a more recent common ancestor with each other than with the rest. Within the polar representatives, isolates from the Antarctic form a group distinct from the Arctic species. Isolate E8 is closer related to the temperate/polar clade than to $\mathrm{NH}_{9}-\mathrm{P}_{7}$ and P. jejudonensis.

Based on the phylogenetic relationships, as well as the genome properties and DDH-based similarities described in Section 3.1, the Octadecabacter clade genomes were divided into 4 groups for subsequent functional analyses and comparisons:

I the polar taxa O. arcticus and O. antarcticus, as well as the novel polar isolates PAMC 27224 \& PAMC 27225

II the temperate taxa O. temperatus and O. ascidiaceicola

III the phylogenetically more distant $P$. jejudonensis and isolate $\mathrm{NH}_{9}-$ $\mathrm{P}_{7}$

IV the temperate isolate E8

Reference clades for the analysis of pan- and core-genome trends were obtained by searching the Roseobacter group phylogeny (Figure A.2) for clusters, which branched at a similar distance from the root as the Octadecabacter clade, and contained a similar number (from seven to twelve) of representatives. Four such clades, encompassing a total of 36 representatives, were chosen for comparison, and their members are listed in Table 3.2.

In order to obtain a higher phylogenetic resolution within the Octadecabacter clade, and to test for ambiguities in their relationships, a consensus cluster network representation of 1,513 individual singlecopy core-gene phylogenies was constructed. Furthermore, the nine genomes were clustered based on their pairwise Jaccard-distances in gene presence and absence. Both trees are presented in the form of a tanglegram in Figure 3.3. 


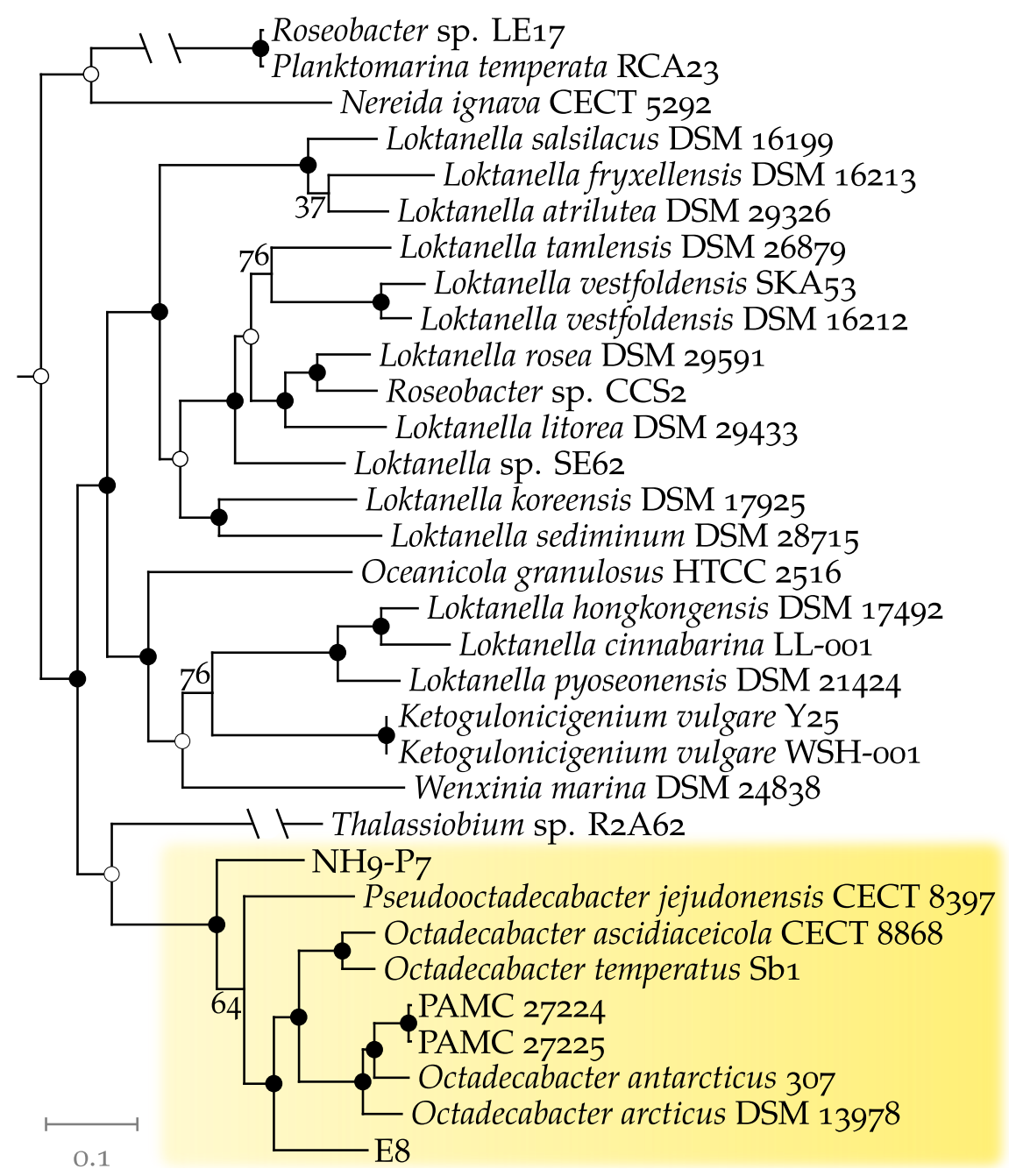

Figure 3.2: MLSA phylogeny encompassing the Octadecabacter clade and its closest relatives. Shown is a subtree pruned out of the larger phylogeny inferred for 115 genomes of the Roseobacter group (given in Figure A.2, p. 131). Numbers indicate bootstrap support, with open and closed circles representing values of 90-99 and 100, respectively. Scale bar in expected number of substitutions. Long edges were shortened by $50 \%$, and are marked with interruptions. The Octadecabacter clade is highlighted.

The network mostly agrees with the MLSA phylogeny in Figure 3.2, but exposes an ambiguous positioning of $O$. antarcticus. A significant amount of single gene phylogenies puts this strain in closer relation to $O$. arcticus than to the PAMC isolates. The internode certainty (IC) and tree certainty (TC) value associated with the respective bipartition are both close to zero, which indicates that both branching patterns occur in a similar number of genes. Relating $O$. antarcticus closer to the PAMC isolates is slightly more frequent than the alternative, as it is associated with a positive IC score. Beside this ambiguity, formation of a polar clade is highly supported (IC score close to 1). O. ascidiaceicola, in agreement with DDH and MLSA, is related closest to O. temperatus at 
Table 3.2: Four monophyletic groups (clades), and their associated Roseobacter group genera, which branch at a similar distance from the root as the Octadecabacter clade. Corresponding members are listed for each clade, and were used for comparative pan-, and core-genome analyses (Section 3.3.1).

\begin{tabular}{cl}
\hline ASS. GENUS & \multicolumn{1}{c}{ GENOMES } \\
\hline Loktanella & L. sediminum DSM 28715, L. koreensis DSM 17925, \\
& Loktanella sp. SE62, L. litorea DSM 29433, \\
& Roseobacter sp. CCS2, L. rosea DSM 29591, L. \\
& vestfoldensis DSM 16212, L. vestfoldensis SKA53, L. \\
& tamlensis DSM 26879, L. atrilutea DSM 29326, L. \\
& fryxellensis DSM 16213, L. salsilacus DSM 16199 \\
Sulfitobacter & S. guttiformis KCTC 32187, S. donghicola KCTC 12864, \\
& Oceanibulbus indolifex HEL-45, S. mediterraneus \\
& KCTC 32188, S. geojensis MM-124, \\
& Sulfitobacter sp. NB-77, Sulfitobacter sp. NB-68, \\
& Roseobacter sp. GAI101, S. pontiacus 3SOLIMARo9 \\
& R. nubinhibens ISM, R. atlanticus R12B, \\
& Roseobacter sp. AzwK-3b, \\
& Roseovarius sp. MCTG1562b, Roseovarius sp. 217, R. \\
& mucosus DSM 17069, Roseovarius sp. TM1035 \\
& P. gallaeciensis ANG1, L. aquimarina DSM 24565, L. \\
& methylohalidivorans DSM 14336, L. caerulea \\
& DSM 24564, L. aquaemixtae CECT 8399, \\
& Rhodobacterales sp. Y4I, L. daeponensis DSM 23529, \\
& Roseobacter sp. SK209-2-6, Pseudophaeobacter arcticus \\
& DSM 23566, Roseobacter sp. MED193 \\
\hline \multirow{2}{*}{ Leisingera } &
\end{tabular}

high IC support. Common ancestry of O. temperatus, O. ascidiaceicola, and the polar clade is less supported than indicated by its bootstrap value in the MLSA phylogeny (Figure 3.2). Likewise, the positioning of isolate E8 directly outside this clade is only slightly more frequent in the individual phylogenies than alternative bifurcations (indicated by a low IC score).

The content-based hierarchical clustering shows a topology similar to the network. Notably, O. arcticus and O. antarcticus are grouped together, and the PAMC isolates form a separate group inside the polar cluster. 


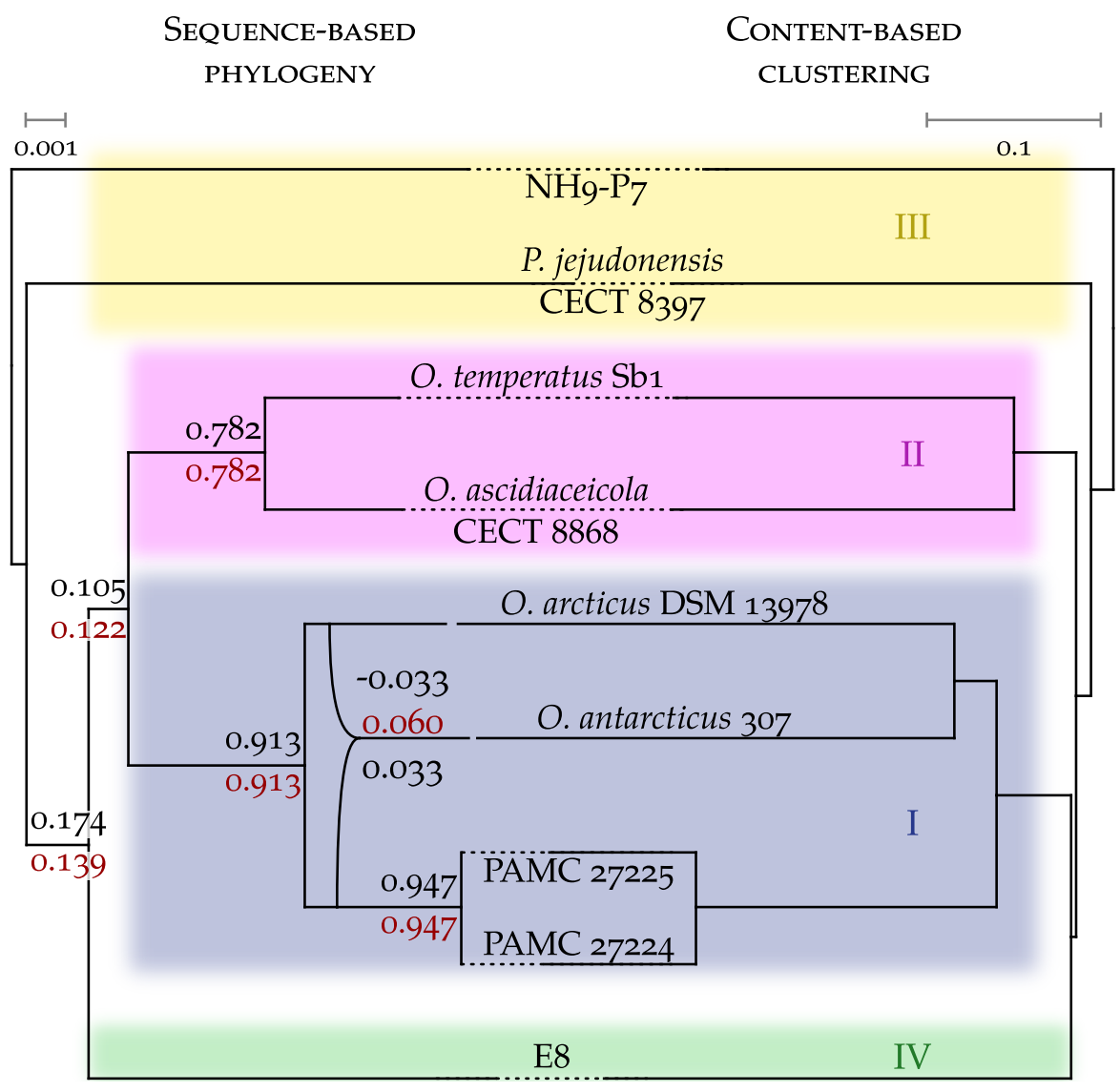

Figure 3.3: Linking core-gene phylogenies to gene content in the Octadecabacter clade. The left is a consensus cluster network representation $(\geqslant 20 \%$ frequency) of 1,513 individual Octadecabacter core-gene phylogenies. Note the ambiguous positioning of $O$. antarcticus. Black and red numbers are IC and TC scores, respectively. The right presents a NJ tree computed from the pairwise Jaccard-distances in gene presence and absence. See Section 2.7 for methods. Scales in expected number of substitutions (left) and distance (right). Both trees were manually rooted, using isolate $\mathrm{NH}_{9}-\mathrm{P}_{7}$ as outgroup. Coloured backgrounds and roman numerals indicate groups for gene content comparison (see main text).

\subsection{GENERAL GENE CONTENT}

\subsubsection{Pan-genome analysis}

Pan- and core-genome sizes by number of included genomes are shown in Figure 3.4 for the Octadecabacter clade. The fitted power law curve, with an exponent of 0.512 , does not reach saturation. Their extrapolated core-genome size is 1,496 . Table 3.3 provides standard deviations (SDs) for both parameters, as well as fitting results for lineages within other Roseobacter group genera, which branch at a similar phylogenetic depth. Over all roseobacter genomes used in this study, the pan-genome exponent is 0.66 , and the estimated core-genome size is 287 (Figure 3.5, see bottom of Table 3.3 for SDs). Concerning the latter analysis, it should be 
noted that the sample size of 600 at each number of genomes is still representative of the underlying distribution, although small compared to the number of possible combinations (e.g. $\left(\begin{array}{c}114 \\ 60\end{array}\right) \approx 13 \cdot 10^{33}$ ) [188].

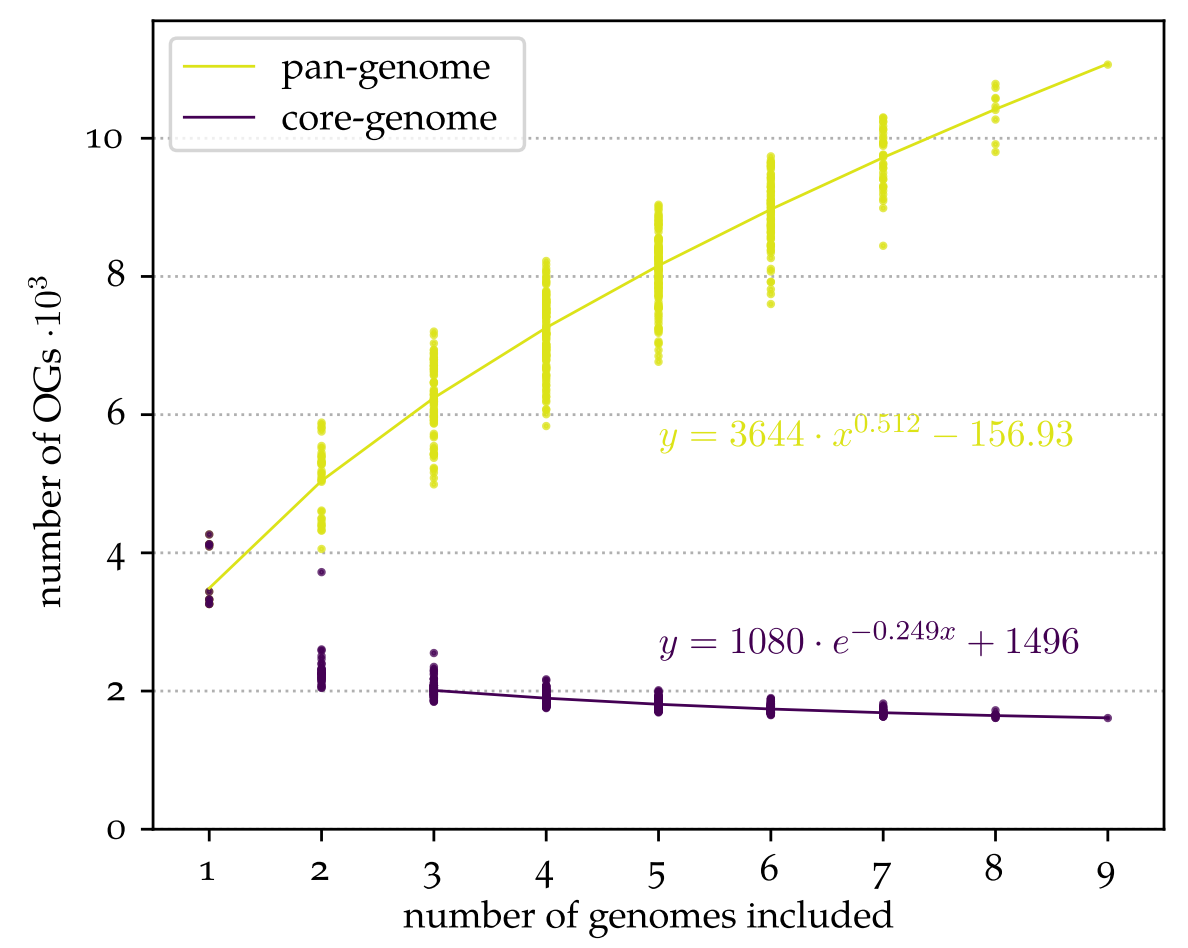

Figure 3.4: Number of pan- and core-OGs for all possible permutations of the indicated number of genomes from the Octadecabacter clade. Curves were fitted based on the median values.

Table 3.3: Exponent $\mathrm{b}$ of the pan-genome, and extrapolated core-genome size of the core-genome curve fits are provided for the Octadecabacters, as well as lineages within other Roseobacter group genera (see Table 3.2). Values represent estimate $\pm S D$. $n$ : number of genomes

\begin{tabular}{rccc}
\hline ASS. GENUS & $\mathrm{n}$ & $\mathrm{b}$ & CORE \\
\hline Octadecabacter & 9 & $0.51 \pm 0.03$ & $1,496 \pm 17$ \\
Loktanella & 12 & $0.644 \pm 0.025$ & $1,162 \pm 20$ \\
Sulfitobacter & 9 & $0.632 \pm 0.018$ & $1,477 \pm 24$ \\
Leisingera & 9 & $0.526 \pm 0.024$ & $2,027 \pm 34$ \\
Roseovarius & 7 & $0.525 \pm 0.025$ & $1,357 \pm 369$ \\
\hline all genomes & $114^{\mathrm{a}}$ & $0.660 \pm 0.001$ & $287 \pm 6$ \\
\hline a as in Table 2.2, excluding Parvularcula bermudensis (outgroup \\
genome), and Phaeobacter sp. LSS9
\end{tabular}

Koonin and Wolf [189] propose to divide the pan-genome into three categories, based on its frequency in the constituent genomes: 

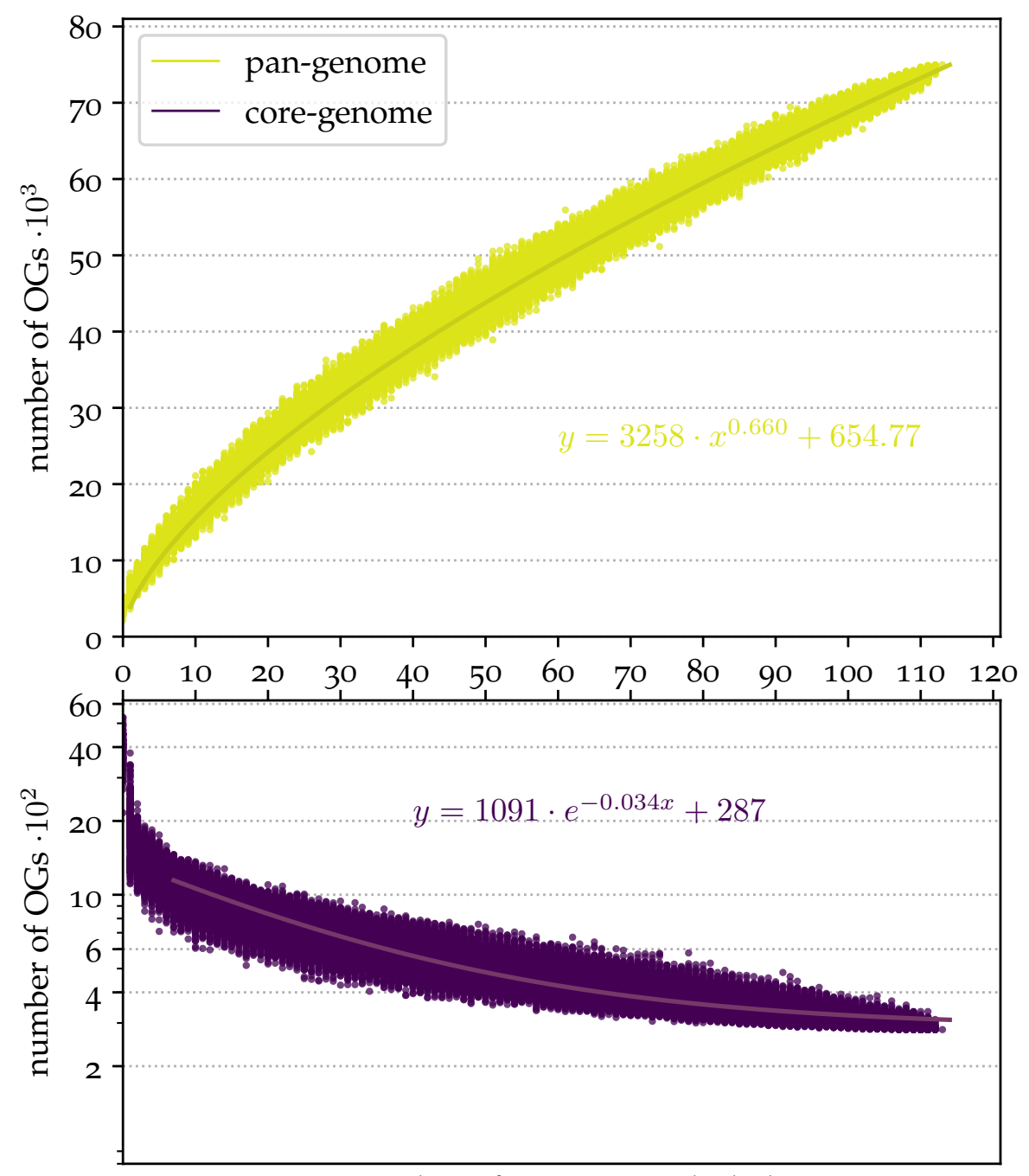

number of genomes included

Figure 3.5: Sizes of pan- (top) and core-genome (bottom) for at least 600 random combinations of the indicated number of genomes from the Roseobacter group (Table 2.2). Curves were fitted based on the median values.

$i$ the core-genome, consisting of genes present in all or the vast majority of comparison organisms,

ii the cloud-genome, containing genes which are less frequent than the core-genome, but occur in multiple of the comparison genomes, and

iii the shell-genome, i. e. genes exclusive to one or a few organisms.

In the Octadecabacter clade, with nine sequenced representatives, sensible boundaries for these categories are $n=9$ for the core-, $1<n<9$ for the cloud-, and $n=1$ for the shell-genome, with $n$ being the number of genomes sharing a respective OG. Absolute CDS counts of each category are provided in Figure 3.6 for each compared genome individually. In all cases, the core-genome, at 1,609 OGs, makes up the 
largest respective fraction of CDSs. The polar Octadecabacter genomes are larger and contain, on average, $\approx 800$ more CDSs than the temperate strains (apparent also from Table 3.1). Most of these CDSs fall into the shell-genome, i. e. are exclusive to a single representative (note that this observation is partially masked in the PAMC-isolates by their high pairwise similarity, i. e. close relation, to each other). O. arcticus possesses the largest absolute shell-genome among the currently sequenced representatives (making up $\approx 30 \%$ of its CDSs).

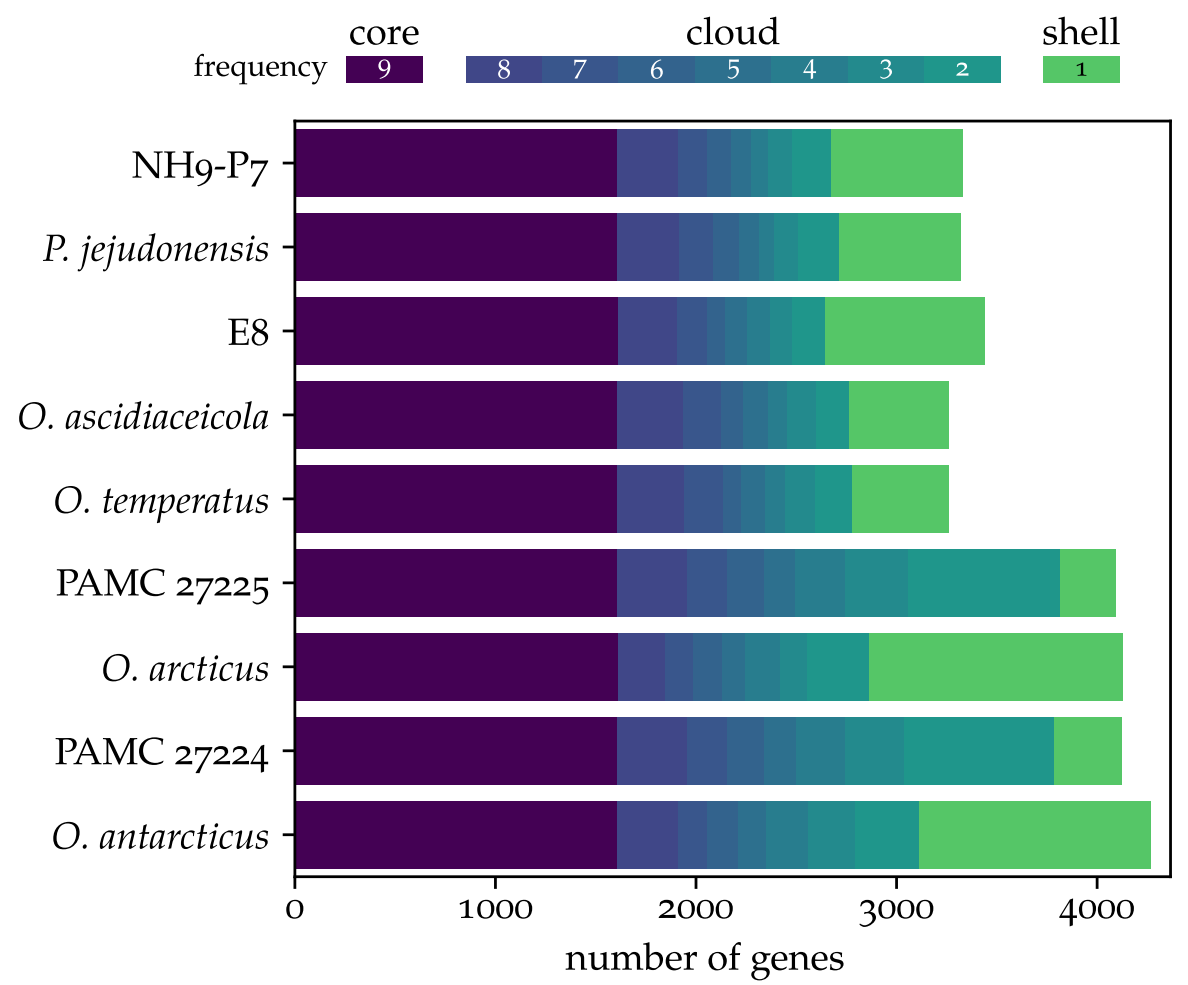

Figure 3.6: Genome category sizes (in number of unique genes, i. e. discounting paralogues) for each Octadecabacter clade genome. Category boundaries in number of genomes $n$ were $n=9$ for core-, $1<n<9$ for cloud-, and $n=1$ for shell-genome.

\subsubsection{Distribution of COG categories}

The number and frequency of genes in different COG categories were analysed for the four Octadecabacter clade groups defined in Section 3.2. The fact that polar Octadecabacter clade members (group I) possess larger genomes than the others (Table 3.1) has to be taken into account when performing a direct comparison. Generally, three distinct trends of COG category size change are possible by comparing larger with smaller genomes:

$i$ the number of genes in a category may remain the same, or decrease, which concomitantly decreases this category's fraction of 
the total COG content (which will be referred to as a negative size correlation from here on),

ii the number of genes in a category may increase linearly with the total number of genes, and this category will therefore occupy the same genome fraction in small and large genomes, and

iii the number of genes in a category may increase super-linearly with the total number of genes, leading to this category occupying a larger fraction of the total COG content.

Both, absolute numbers and category fractions are provided for the four groups in Figure 3.7. Visually, each COG category was assigned to one of the three behaviours described above, summarised in Table 3.4.

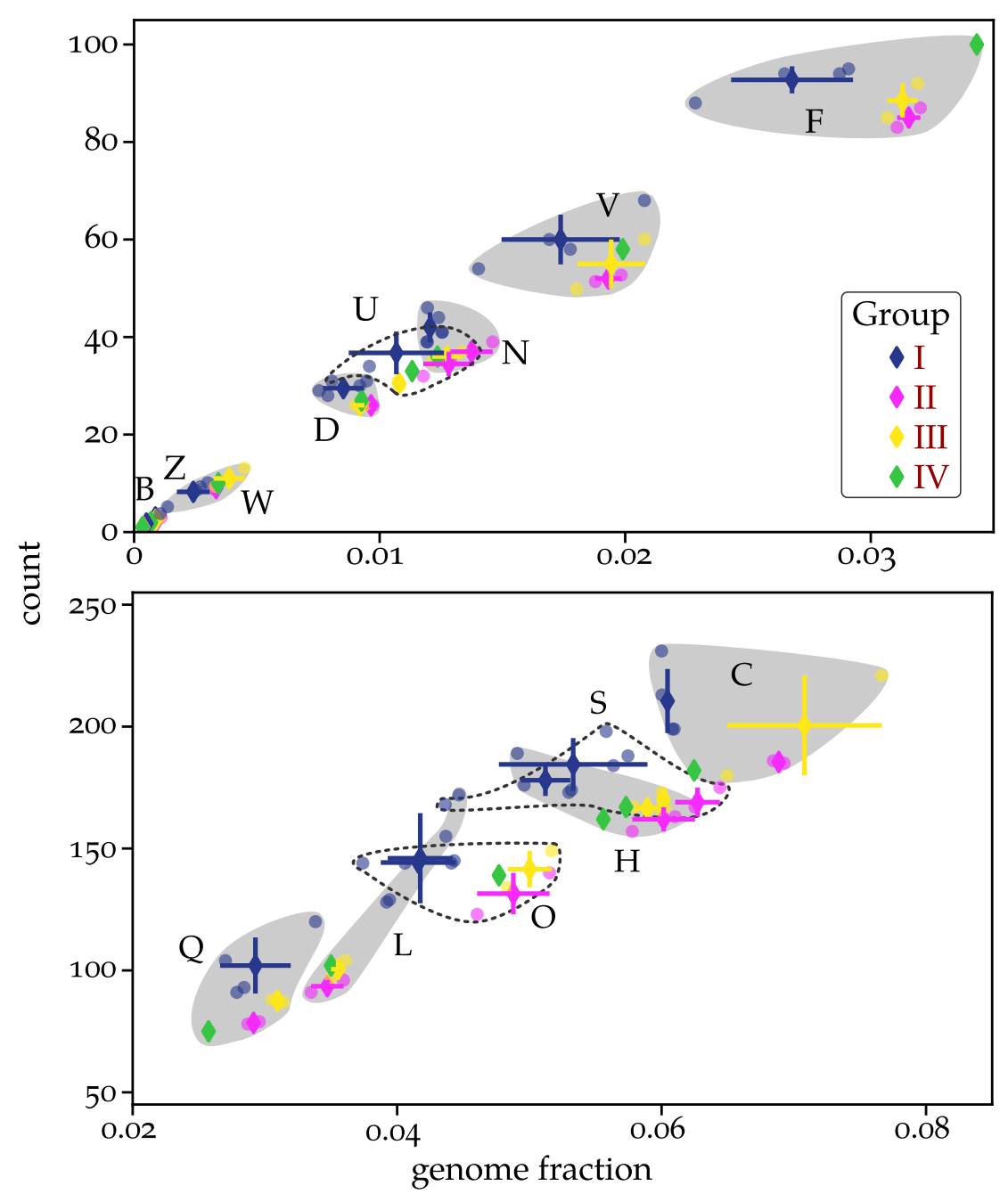

(a) COG categories B, C, D, F, H, L, N, O, Q, S, U, V, W, and Z

Figure 3.7: Mean absolute numbers of COGs in each category are plotted against its fraction of total COG content as diamonds. Dots show individual values of the constituent genomes. Groups are as defined in Section 3.2. Bars indicate $\mathrm{SD})$. 


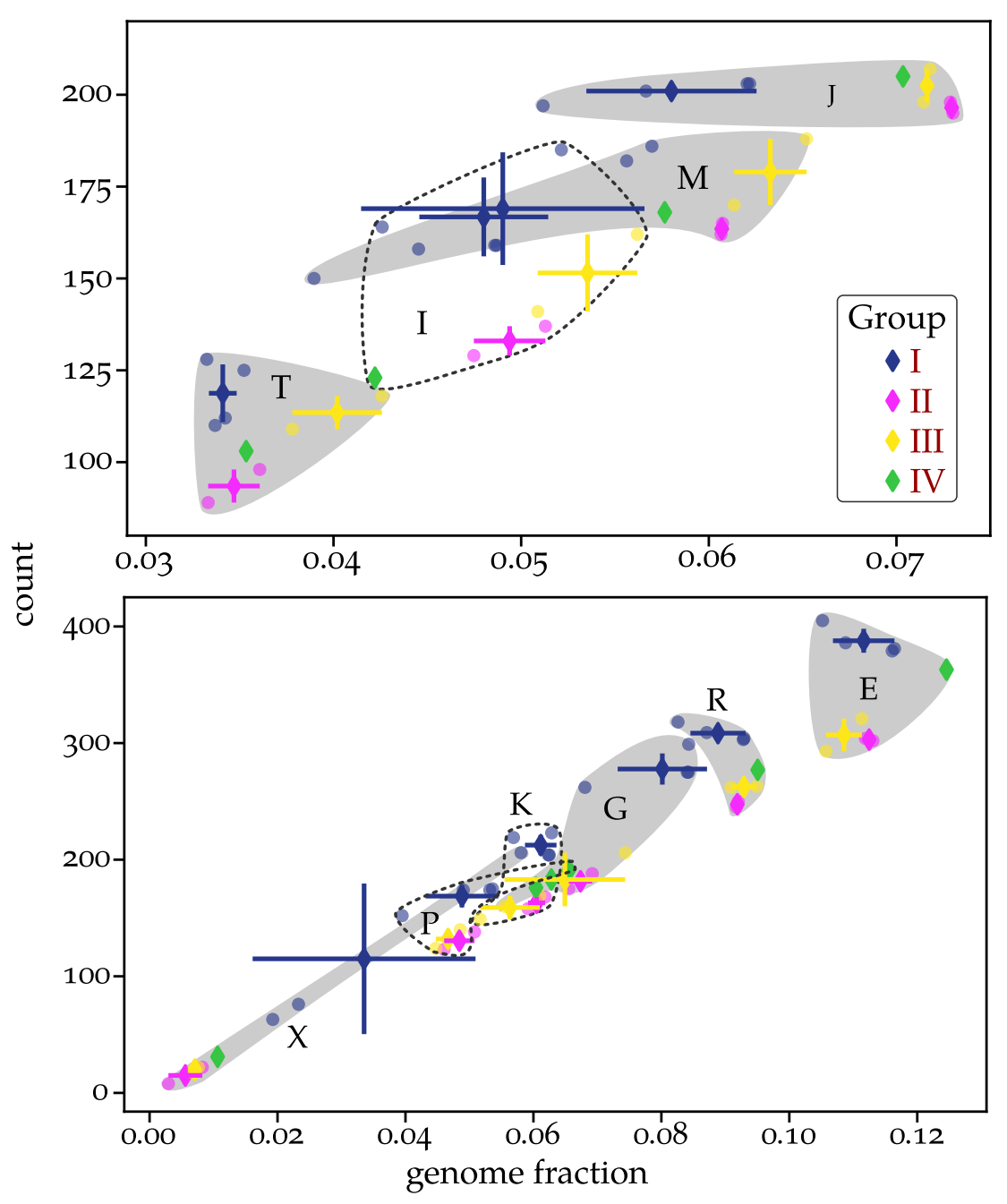

(b) COG categories E, G, I, J, K, M, P, R, T, and X

Figure 3.7: COG category distributions in the Octadecabacters (continued)

Table 3.4: COG category trends in polar Octadecabacter clade genomes compared to the temperate strains. Trends were derived from Figure 3.7. For their definitions, see the main text.

negative correlation with genome size

C Energy production and conversion

F Nucleotide transport and metabolism

$\mathrm{H}$ Co-enzyme transport and metabolism

J Translation, ribosomal structure, and biogenesis

M Cell wall, membrane, and envelope biogenesis

O Posttranslational modification, protein turnover, and chaperones 
Table 3.4: continued

\begin{tabular}{ll}
\hline S & Function unknown \\
\hline & \multicolumn{1}{c}{ linear increase with genome size } \\
\hline E & Amino acid transport and metabolism \\
I & Lipid transport and metabolism \\
$\mathrm{K}$ & Transcription \\
$\mathrm{N}$ & Motility \\
$\mathrm{P}$ & Inorganic ion transport and metabolism \\
$\mathrm{Q}$ & Secondary metabolite biosynthesis, transport and metabolism \\
$\mathrm{R}$ & General function prediction only \\
$\mathrm{T}$ & Signal transduction mechanisms \\
$\mathrm{U}$ & Intracellular trafficking, secretion, vesicular transport \\
$\mathrm{V}$ & Defence mechanisms \\
\hline & \\
\hline $\mathrm{G}$ & Carbohydrate transport and metabolism \\
$\mathrm{L}$ & Replication, recombination, and repair \\
$\mathrm{X}$ & Mobilome (prophages, transposons) \\
\hline & \\
\hline $\mathrm{Z}$ & Cy \\
\hline $\mathrm{D}$ & Chromatin structure and dynamics \\
$\mathrm{W}$ & Extra-cellular structures \\
\hline
\end{tabular}

\section{$3 \cdot 4$ GROUP-SPECIFIC GENES}

specific - present in all members of one group and absent in all other groups
Figure 3.8 gives an overview of the number of genes specific to each group defined in Section 3.2, and any of their combinations. po2group stats.pl estimates a core-genome size of 1,609 for the nine genomes. At 90, group I (polar Octadecabacters) has the highest number of specific genes (discounting the 795 accessory genes of isolate E8). Paralogous genes in the reference genome, listed by po2group_stats.pl as multiple entries, were only counted once. The individual genes specific to each group are provided in Tables A.2 to A.5, Appendix A.4, p. 135.

Fifty-five of the 90 genes specific to polar Octadecabacters are colocalised at 10 positions, with cluster sizes ranging from 2 to 14 . Sev- 
enteen genes are annotated as hypothetical proteins (or their functions are not clearly specified), and 5 as transposases or recombinases.

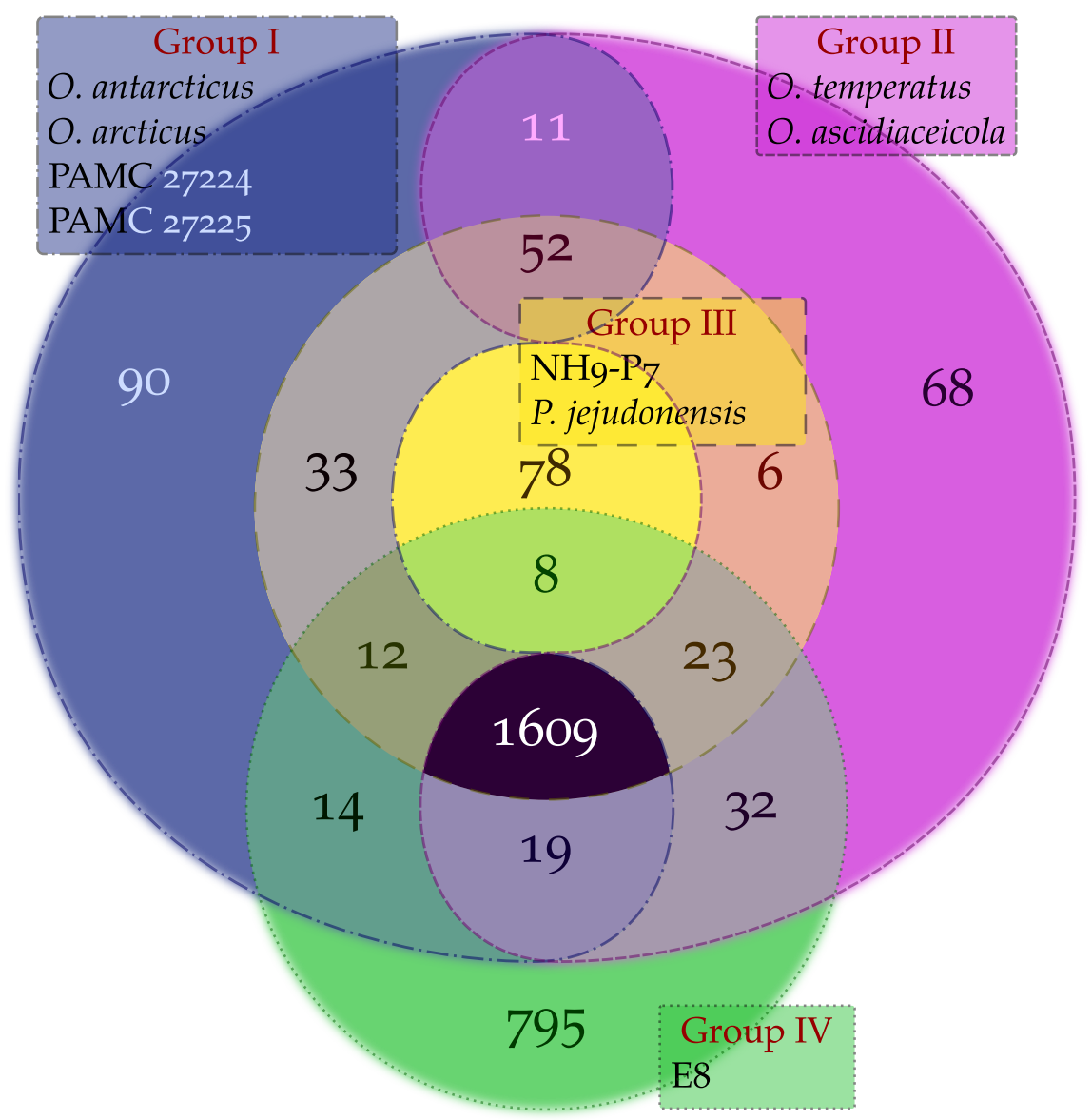

Figure 3.8: 4-set Venn diagram showing number of genes shared between and specific to each group defined in Section 2.10.2.

Cases in which multiple genes are encoded directly adjacent to each other, in conserved gene clusters, are of particular interest, as adjacency often entails functional interaction or interrelation [190]. Therefore, these cases will be discussed concomitantly in the following sections.

Since the adaptation of polar Octadecabacters (group I) to their extreme habitat is of main interest, focus will be placed on the description and interpretation of genes specific to this group.

Comparison group IV consists of isolate E8 as the only member, and its 795 specific genes therefore constitute the accessory genome of this strain. As a generalisation from one individual is not meaningful, discussion of these genes will be omitted here.

\subsubsection{Genes specific to polar Octadecabacters}

As stated above, 55 of the 90 genes specific to polar Octadecabacters are co-localised at 10 positions. The corresponding gene products include 
several transcriptional regulators such as MarR, stress-related proteins, a xanthorhodopsin, dehydrogenases, and enzymes for the degradation of various substrates (Figures 3.9 to 3.18). Seventeen genes are either annotated as hypothetical proteins, or their functions are not clearly specified.

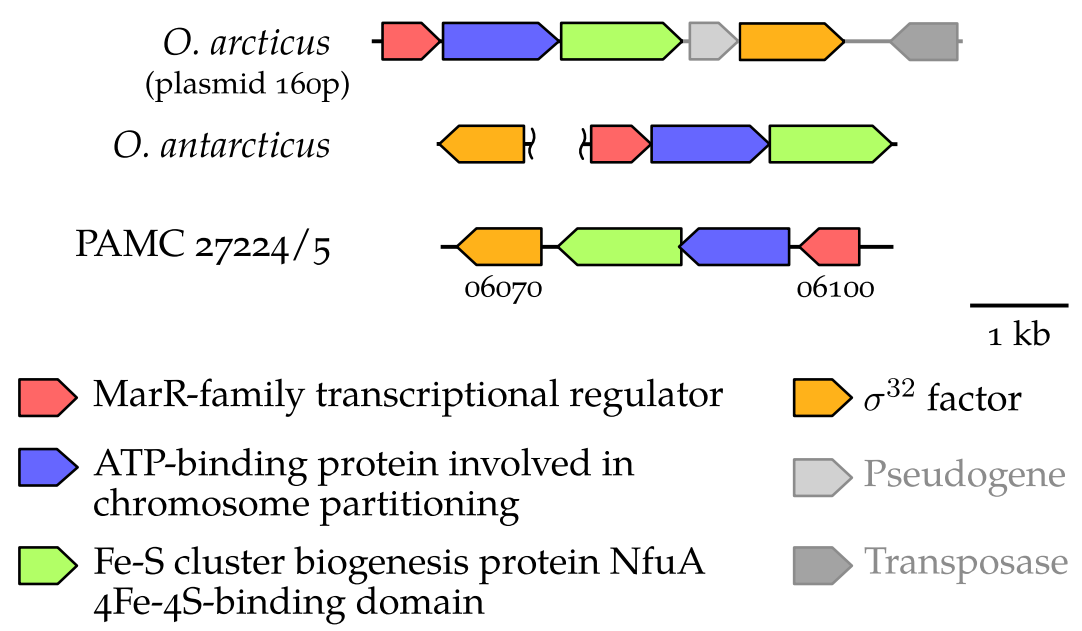

Figure 3.9: MarR-associated gene cluster in polar Octadecabacters

Polar Octadecabacter-specific regulatory proteins include a member of the MarR-family (Figure 3.9). MarR trancriptional regulators are associated, among other factors, with oxidative stress in E. coli [191]. The corresponding gene is usually found adjacent and divergently transcribed to the target genes it regulates, and through binding the intergenic region, it represses both its own and its targets' transcriptions [191]. The current model of its inactivation through oxidative stress is as follows: oxidation of one or multiple MarR cysteine residues leads to conformational changes, which impair DNA-binding of this repressor and subsequently lead to transcription (activation) of its target operon [191]. The corresponding oxidative stress signal was recently found to be brought about by copper ions, released upon cell envelope stress, in E. coli [192]. MarR may also be involved in metabolic regulation, where it is activated by binding to a specific substrate, and consequently acts as transcriptional activator of the respective catabolic pathway, binding upstream of the respective genes [193].

It has not yet been experimentally tested whether the conserved, polar Octadecabacter-specific cluster formed by MarR and its three neighbouring genes is actually under MarR regulation. However, the corresponding gene functions, which are involved in stress response indicate this: in cases in which it is induced by stress, MarR is often associated with efflux pumps [191], and the downstream neighbouring gene octa_0609o has $>70 \%$ identity to a sodium:proton antiporter in a reference member of the Rhodobacteraceae. One of the factors severely affected by oxidative stress is sulphur biochemistry [49], and fittingly, the next downstream neighbour in polar Octadecabacter MarR-gene clus- 
ters encodes an NfuA-like gene product. NfuA assists FeS-cluster biogenesis, likely by binding newly synthesised FeS-clusters and shuttling them to their targets [194]. It can thus shield these FeS-clusters from the intracellular environment, thereby protecting them from oxidative stress [194]. For example, aconitase B of the tricarboxylic acid (TCA) cycle is one important NufA target in E. coli [194].

The final gene found in conjunction with this MarR-associated gene cluster is a sigma factor related to $\sigma^{32}$, although it is not always in direct neighbourhood. This sigma factor is involved in the heatshock response of $E$. coli, but also regulates transcription of additional genes [195]. For polar Octadecabacters, it may exert a protective function by inducing expression of chaperones. Notably, it may also be involved in maintenance of proper sulphur biochemistry through induction of thioredoxin expression [195].

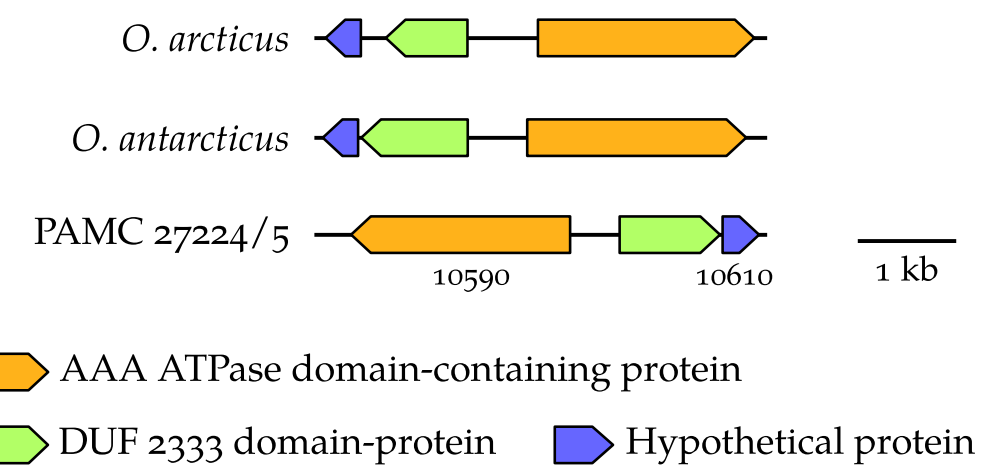

Figure 3.10: AAA ATPase specific to polar Octadecabacters

A potentially stress response-associated gene specific to polar Octadecabacters is an AAA ATPase (Figure 3.10). These are found in all domains of life and generally induce conformational changes in proteins upon adenosine triphosphate (ATP)-phosphorylation [196]. They act in a wide range of cellular processes, most notably in protein unfolding at the proteasome lid, dis-assembly of protein aggregates and complexes, and membrane protein extraction for subsequent degradation [196]. This unfolding property may be particularly important for polar Octadecabacters, due to the low resident temperatures of their habitats. As described in Section 1.2, the cell membrane becomes more rigid at low temperature, which may necessitate this additional factor for proper membrane protein extraction and degradation. Although protein stability is generally decreased as adaptation to low temperatures, the specific properties of some proteins might still require accessory functions for their successful degradation, such as the AAA ATPase.

Another, apparently stress-associated, component found only in polar Octadecabacter genomes affects cellular selenium biochemistry (Figure 3.11). Selenium is an important trace element, which may be incorporated into proteins (mostly in the form of selenocysteine) and nu- 


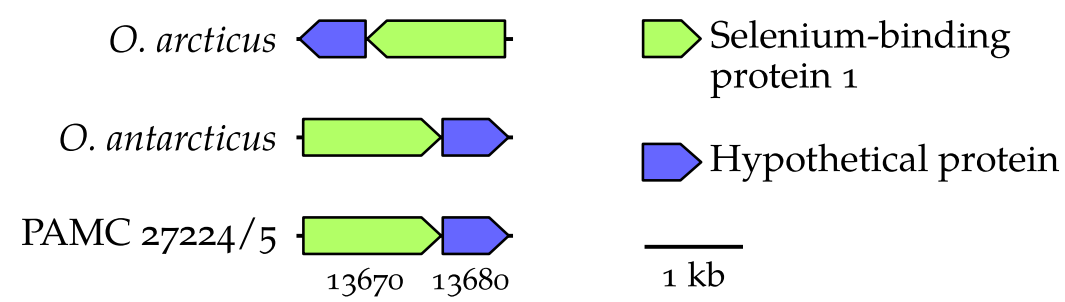

Figure 3.11: Selenium-binding protein specific to polar Octadecabacters

cleotides [197]. All Octadecabacter clade members seem to synthesise selenophosphate (via the product of selD) and to use 2-selenouridinemodified tRNA (sell gene). Free selenium species induce oxidative stress, most importantly by binding glutathione molecules, thereby forming $\mathrm{O}_{2}^{-}$radicals [198]. Detoxification happens via reduction by thioredoxin [197], or, at lower rate, spontaneously-formed elemental selenium $\left(\mathrm{Se}^{\mathrm{O}}\right)$. Selenium-binding proteins (SBPs) may bind free selenium species through exposed thiol groups and shuttle them to selenophosphate synthase, thereby shielding them from the cellular environment [197]. However, other thiol-containing cellular enzymes, such as 3-mercaptopyruvate sulfurtransferase (3-MST) and glyceraldehyde-3-phosphate dehydrogenase (GAPDH), bind and release free selenium effectively, and could therefore suffice as shuttles to supply selenophosphate synthase with selenium [197, 199]. The function of SBPs consequently remains elusive to this day [197].

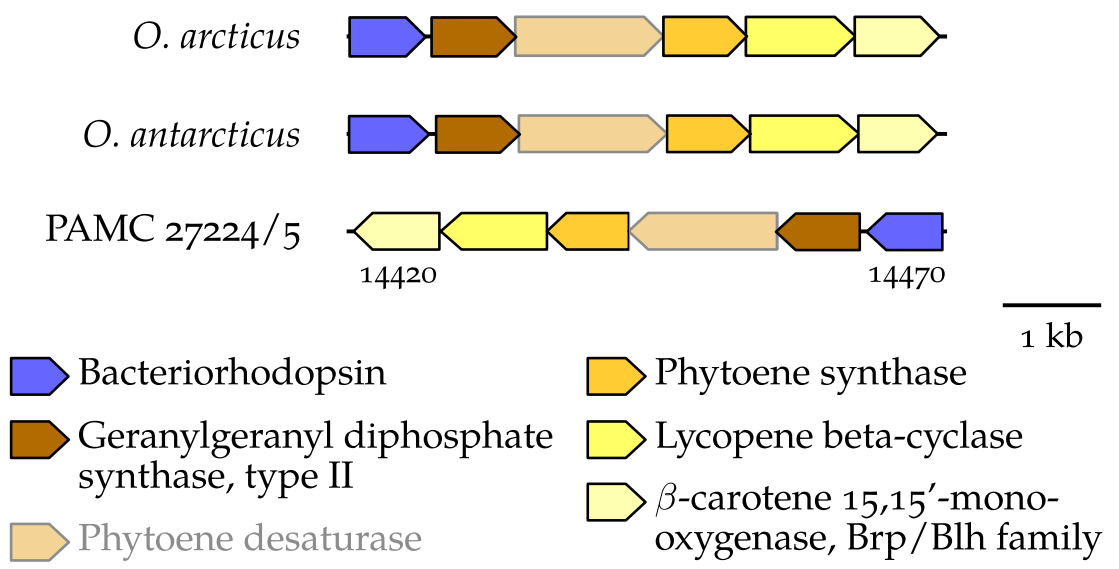

Figure 3.12: Xanthorhodopsin gene cluster specific to polar Octadecabacters

All polar Octadecabacter strains contain a rhodopsin-coding gene cluster (Figure 3.12), enabling potential photoheterotrophic life strategies. Bacterial rhodopsins were originally discovered in a marine fosmid clone [200], and it is now clear that they are more abundant than photochemical reaction centres in most marine habitats [201]. Vollmers et al. described a new subgroup of rhodopsins, the so-called group II xanthorhodopsins in O. arcticus and O. antarcticus, which they associated with cold and saline habitats [77]. The gene locus organisation of 
this group of rhodopsins is also conserved, with the five genes needed for synthesis of the rhodopsin's chromophor retinal localised downstream of the opsin gene [77] Phylogenetic reconstruction of the PAMC isolate-derived rhodopsins (see Figure A.6 on page 168), as well as the organisation of the gene locus (Figure 3.12) revealed that these belong to the same group. The fact that in the present analysis, group II xanthorhodopsins were found to be specific for all polar Octadecabacters, supports the association by Vollmers et al. While this type of phototrophy is relatively rare among Roseobacter group members (eight out of 115 genomes used presently, including one proteorhodopsin), xanthorhodopsins are generally exclusive to polar roseobacters, and occur in the majority of them, as visualised in Figure A.5 (p. 167).

The cellular role of xanthorhodopsins is currently still unclear, and may differ greatly between species [202]. Generally, xanthorhodopsins, like their more abundant sister-group proteorhodopsins, utilise light energy to translocate protons across the cell membrane, thereby generating a proton-motive force [200, 203]. This force could be used for ATP-synthesis, ion transport, or flagellar propulsion [202]. Importantly, in E. coli, proteorhodopsin was not able to create a greater membrane potential than the respiratory chain [204], consequently not adding to the proton-motive force and the observed growth speed. However, it was able to recover the membrane potential when the respiratory chain was blocked, implying a protective function for the cell during respiratory stress [204].

Experiments on Vibrio species implied involvement of proteorhodopsin in starvation survival [205], and Vollmers et al. hypothesised a similar function for the xanthorhodopsins of the sea ice residents $O$. arcticus and O. antarcticus [77].

The largest gene cluster specific for polar Octadecabacters was found to consist of 15 syntenic genes in the PAMC isolates, but two separate regions, respectively, in O. arcticus and O. antarcticus (Figure 3.13). In this cluster, a LacI-family transcriptional regulator is found adjacent and divergent to multiple partly overlapping genes, indicating that the cluster constitutes an operon. This family usually regulates carbohydrate metabolism [206], which concurs with the downstream presence of a CUT 1 family carbohydrate ABC-transporter. The CUT 1 family may transport a broad range of substrates, such as polyols, oligosaccharides, or glycerol-phosphate [207]. An adjacent dihydroxyacetone (DHA) kinase is involved in glycerol degradation [208]: DHA is formed from glycerol through the action of a dehydrogenase, and subsequently phosphorylated to form DHA phosphate (DHAP), a glycolysis intermediate. Notably, the Octadecabacters do not possess the respective dehydrogenase (instead, all Octadecabacters degrade glycerol via phosphorylation using a glycerol kinase, and subsequent oxidation to DHAP). Instead, DHA may be imported from the environment (e. g. through the $\mathrm{ABC}$-transporter in question), as has been observed for other hyper- 
O. arcticus

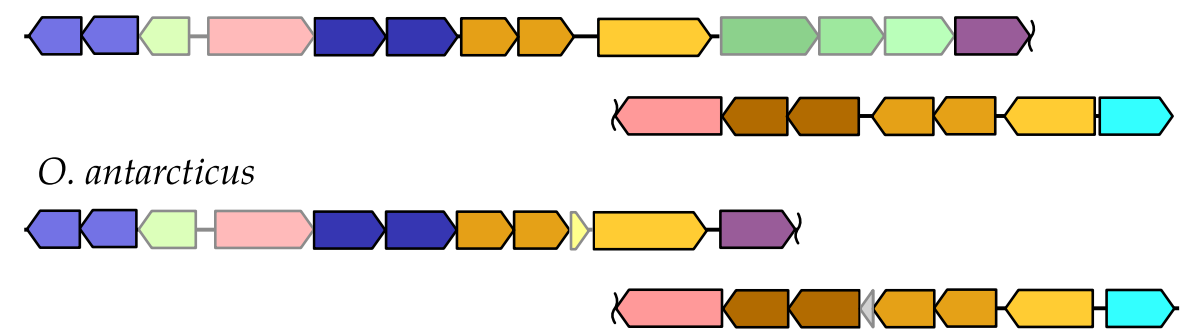

PAMC 27224/5

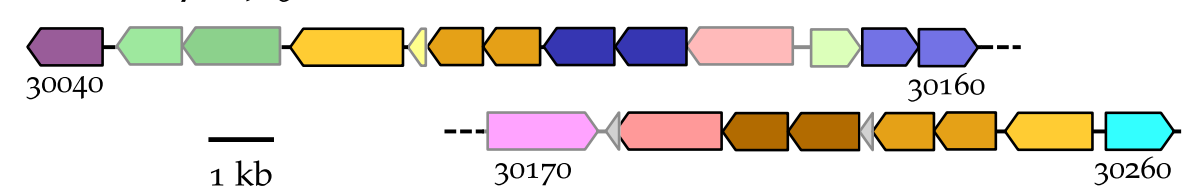

Alcohol dehydrogenase class IV

D-fructose 1,6-bisphosphatase

Glycerol kinase

$>>$ carbohydrate ABC-transporter, CUT 1 family

Predicted small integral membrane protein

$>>$ Glycerol transport system

Glycerol-3-phosphate dehydrogenase

$>$ DeoR-family transcriptional regulator

$D$ Choline/carnitine/ betaine transport

Hypothetical protein

Dihydroxyacetone kinase

LacI-family transcriptional regulator

Fructose-bisphosphate aldolase

Figure 3.13: Cluster of genes associated with uptake and degradation of carbohydrates, specific to polar Octadecabacters

saline communities: in short, primary producers synthesise glycerol as osmoprotectant, which heterotrophs then take up for degradation. A part of the imported glycerol may be secreted again, after oxidation, as DHA [209]. DHA thus constitutes an overflow product [209]. The situation may be similar in the sea ice habitats of polar Octadecabacters, since they live in close association with primary producers, and salinity often reaches near-saturation levels in brine channels (Section 1.2).

The remaining genes in this region possess similar functions: subunits of a glycerol ABC-transporter are localised beside an incomplete CUT 1 system, and both are specific to polar Octadecabacters. Furthermore, with the presence of a glycerol kinase and a glycerol-3-phosphate dehydrogenase, the complete pathway of glycerol degradation is found in this locus, under the control of a DeoR-family transcriptional regulator. The latter three components are not exclusive to polar isolates, as orthologues of these can be found in all Octadecabacter clade genomes sequenced to date. 
In conclusion, this locus seems to be involved in glycerol degradation in polar Octadecabacters. This degradation may also be regulated in response to osmotic and temperature changes, since glycerol is synthesised as both osmo- and cryoprotectant by many algae.

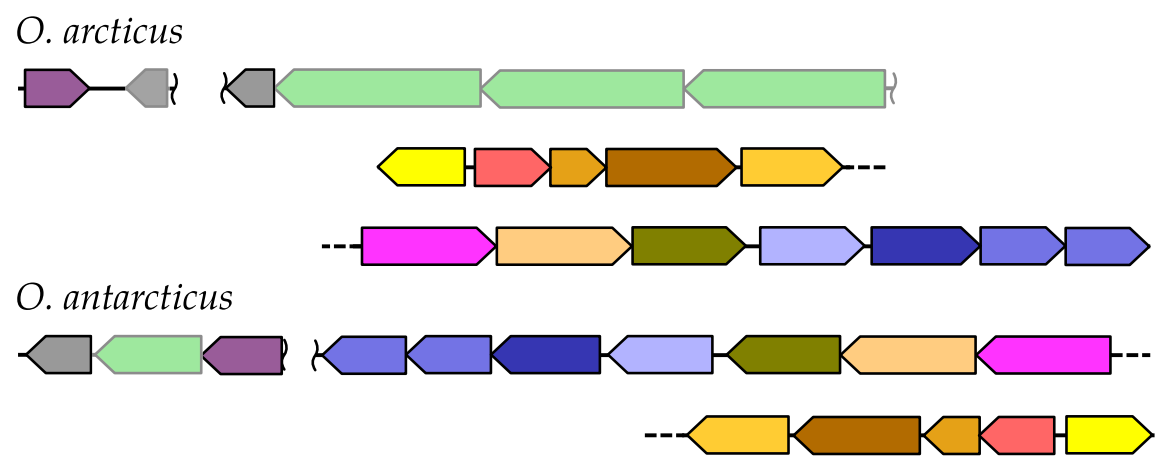

PAMC 27224/5

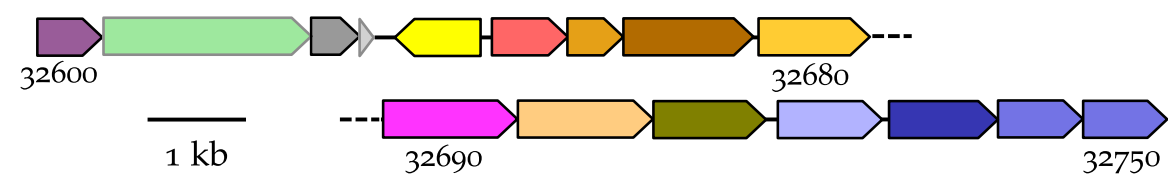

TetR-family transcriptional regulator

$\square$ Dimethylamine/trimethylamine dehydrogenase

Hypothetical protein

$\square$ RpiR-family transcriptional regulator

$\square$ Predicted N-formylglutamate amidohydrolase

$\square$ TRAP-type mannitol/chloroaromatic compound transport system, small permease

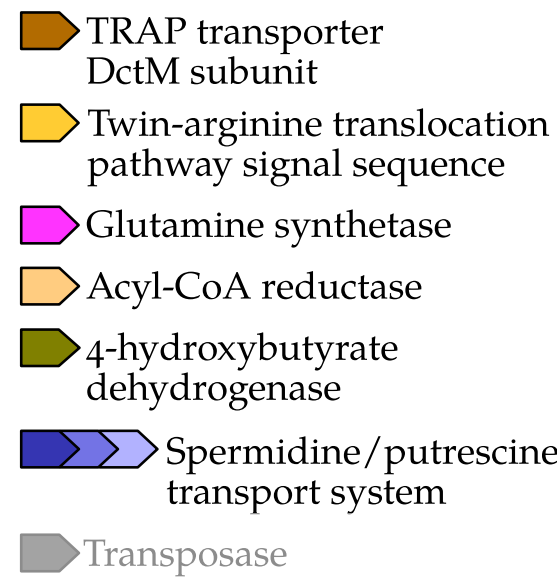

Figure 3.14: RpiR- and TetR-associated gene clusters specific to polar Octadecabacters

Another large cluster specific for polar Octadecabacters was found to consist of a set of eleven syntenic genes, preceded by an inversely transcribed RpiR-family regulator (Figure 3.14).

The role of the transcriptional regulator has not been characterised in this group. RpiR was first described to repress ribose phosphate isomerase B of the pentose phosphate pathway in E. coli [210], whereas it serves as an activator in Staphylococcus aureus [211]. Generally, the family is involved in regulation of sugar catabolism [211, 212]. This could functionally correlate with a downstream tripartite ATP-independent periplasmic (TRAP) transport system encoded in the same gene cluster. The twin-arginine translocation pathway signal sequence is annotated as the $d c t P$ component of a TRAP system in O. arcticus and O. antarcti- 
cus, thus constituting a complete system. These transport a wide range of substrates in different organisms, including ectoin and carboxylic acids [213]. It is important to note that substrate specificity can only reliably be determined in vitro [214]. However, uptake of mannitol, as suggested by automatic annotation, or a similar compound would provide intermediates for use in either glycolysis, or the pentose phosphate pathway.

Apart from the TRAP transporter, genes for a spermidine/putrescine ABC-transport system are also located at this site. All Octadecabacters possess several copies of this transporter, and are able to degrade putrescine via the putrescine utilisation pathway (puu operon [215]), but its corresponding biosynthetic pathway is absent. Polyamines generally provide protection against a number of stress factors [216], but also serve as a nitrogen source. Indicative of a primary role as nitrogen donor in this context is an upstream glutamine synthetase (GS) gene within the same cluster, the product of which constitutes the primary mechanism of nitrogen assimilation [217].

A predicted $\mathrm{N}$-formylglutamate amidohydrolase within the same cluster could catalyse the last step in histidine degradation (see Section 3.5.4 for more detailed information), which yields glutamate and formate. The former serves as substrate for GS, thereby increasing the capacity for nitrogen uptake.

In addition, a TetR-family regulator can be found upstream, in relatively close proximity to this gene cluster in the PAMC isolates, but at varying locations and genomic contexts in the polar Octadecabacter type strains. This family of regulators can affect a wide range of functions, including metabolic homoeostasis [218]. It is, however, most often associated with the regulation of efflux pumps under conditions of cellular stress [218].

In conclusion, the genes in this cluster may be primarily involved in the regulation of purine and pyrimidine synthesis, since both glutamine, and intermediates of the pentose phosphate pathway are required as substrates in these pathways.

Three of the polar Octadecabacter-specific genes were found to lie in close proximity, albeit not directly adjacent to each other, in most of the four genomes (Figure 3.15). Among them is a transporter of the BASS family, which imports (primarily) bile acids in conjunction with sodium ions, and has been well characterised in animals [219]. The family is also known to transport other substrates, such as steroids and their derivatives [219]. Structural data and general characterisations of this family are sparse in prokaryotes [220], and we are consequently lacking detailed information regarding their functions and substrates in this group of organisms. Generally, however, bile salts frequently occur in the environment, and can be used as the sole carbon source by some bacteria, although the genetics and regulation behind this process are currently poorly characterised [221]. Similarly, myo-inositol widely oc- 


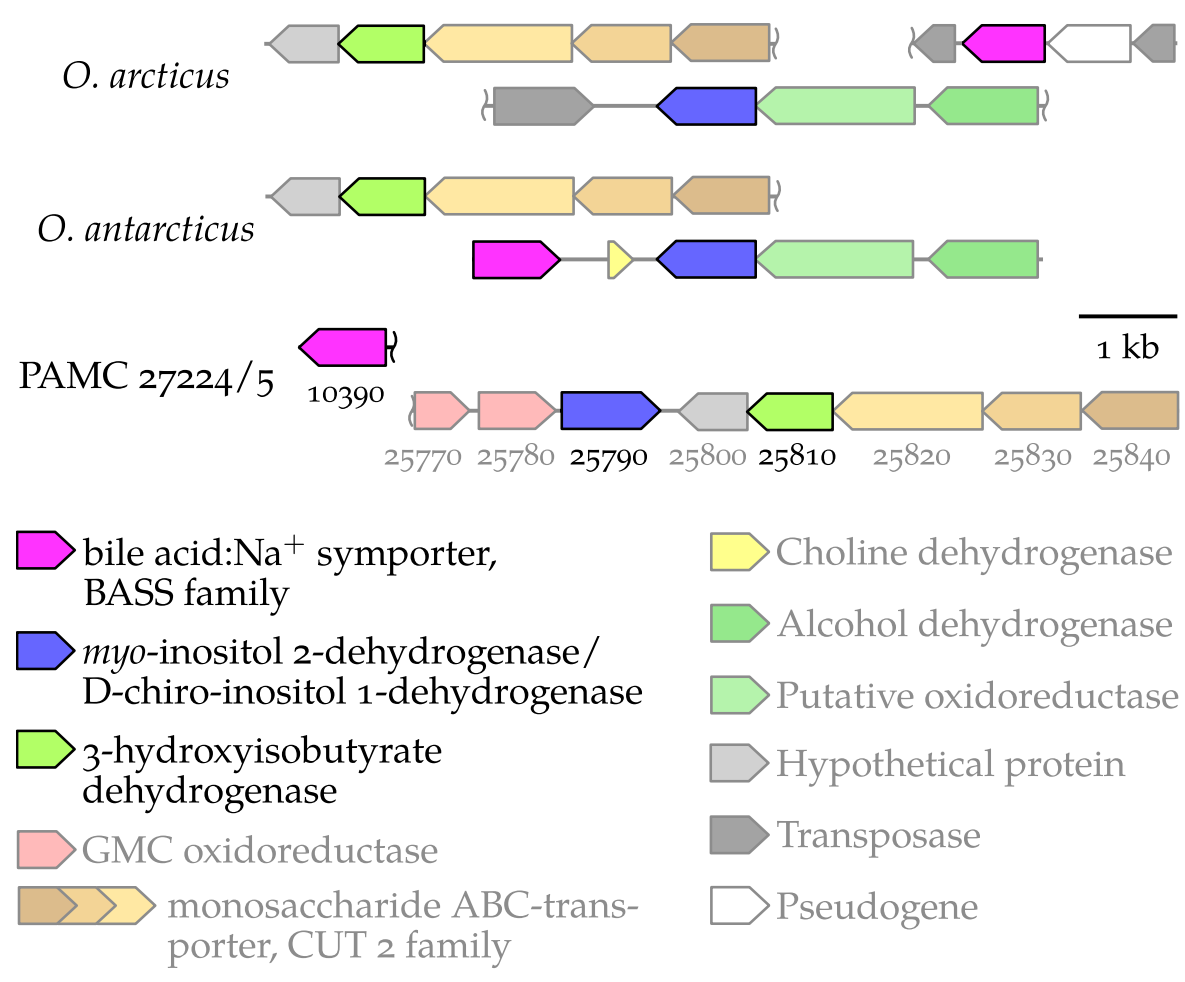

Figure 3.15: Genes specific to polar Octadecabacters, which may be involved in degradation of diverse substrates

curs in natural habitats, and is readily degraded by diverse bacteria to DHAP and acetyl-coenzyme A ( $\mathrm{CoA}$ ) (Ac-CoA) [222-225]. myo-inositol dehydrogenase, which was found to be specific for polar Octadecabacters, catalyses the first step in myo-inositol degradation [226]. Usually, the genes needed for its catabolism are organised in the iol-operon [226], and an orthologous form of this operon is present in all currently sequenced Octadecabacters except for isolates E8 and NH9-P7. The polar-specific dehydrogenase is non-paralogous to its homologue in this operon, and was therefore likely gained by a common ancestor of the polar Octadecabacters through lateral transfer.

Finally, a polar Octadecabacter-specific 3-hydroxyisobutyrate dehydrogenase gene is positioned downstream and adjacent to a (nonspecific) carbohydrate ABC-transporter. Its gene product is involved in valine degradation, which ultimately results in succinyl-CoA. CUT 2family transporters are usually specific for monosaccharides [207], and the transport system found at this location therefore likely is not involved in either valine, or myo-inositol uptake.

Without appropriate experimental examination, a detailed function of these three specific genes remains unknown. However, they generally seem to be involved in the uptake and degradation of carbohydrates, thereby broadening the spectrum of substrates polar Octadecabacters can utilise for their growth. 


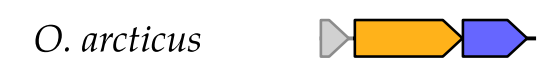

O. antarcticus

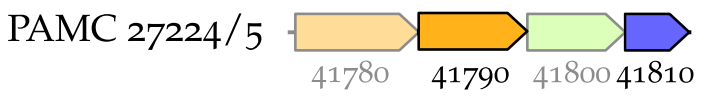

$$
\overline{1 \mathrm{~kb}}
$$

$5^{\prime}$-methylthioribose

kinase

$\square$ L-fuculose-phosphate

aldolase

Methylthioribose-1-phosphate isomerase

Uncharacterized

Transcriptional regulator LsrR, conserved protein

DeoR family

Figure 3.16: $m t n A$ and fucA in polar Octadecabacters

An $m \operatorname{tn} A$ gene (and its immediate genomic context) was also found to be specific for polar Octadecabacter isolates (Figure 3.16). Its product, methylthioribose-1-phosphate isomerase, participates in the methionine salvage pathway, which may not be complete in these organisms (see Section 3.5.3).

In $O$. arcticus and $O$. antarcticus, this gene is directly adjacent to the group-specific fuc $A$, coding for L-fuculose-phosphate aldolase. This enzyme is involved in L-fucose and D-arabinose degradation, and specifically converts L-fuculose-1-phosphate to DHAP [227]. The two genes could therefore broaden the range of substrates polar Octadecabacters can use for growth, thereby reflecting diversity as well as scarcity of nutrients in their natural habitats.

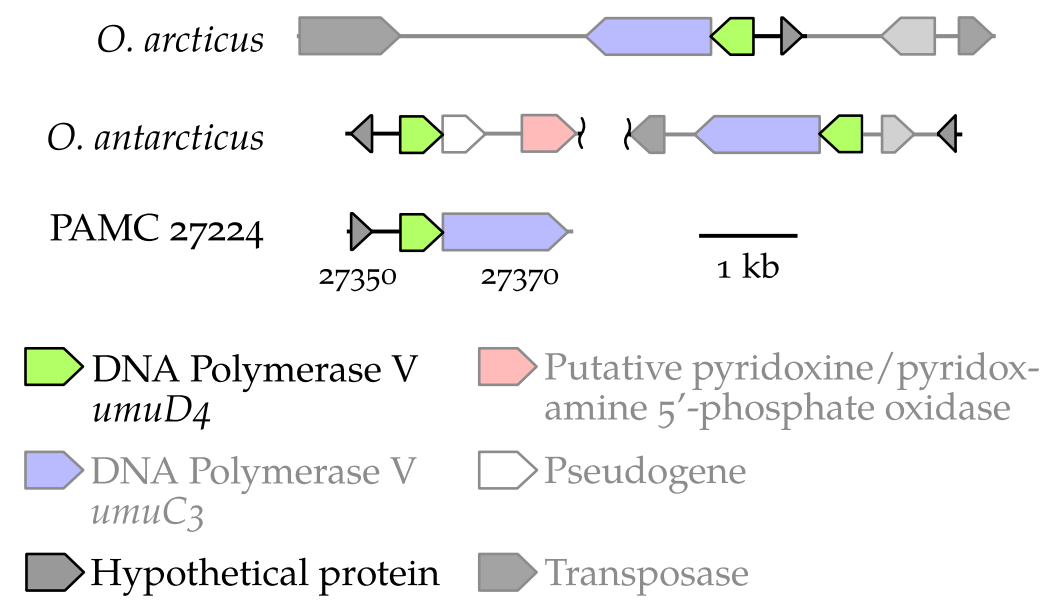

Figure 3.17: Genes encoding DNA polymerase V specific to polar Octadecabacters

Among the OGs specific to polar Octadecabacters are genes coding for a DNA polymerase (Figure 3.17). DNA polymerase $\mathrm{V}$ belongs to the Y-family of polymerases, and is involved in error-prone repair 
of DNA damage, most notably translesion DNA synthesis (TLS) [228]. A heterotrimeric $\mathrm{UmuD}_{2}^{\prime} \mathrm{C}$ complex forms the functional polymerase. While no annotated $u m u C$ gene was found in the PAMC isolate 27225, and this gene can therefore currently not be considered group-specific, this is likely due to the high fragmentation of that genome (Table 3.1).

Generally, DNA Pol V is strongly induced after ultraviolet (UV) irradiation, which is seasonally intense in the polar regions [50], and exacerbated by decreasing atmospheric ozone content [229]. Induction happens at a late stage of the stress response, and this is interpreted as a compromise between its ability to perform TLS and its low fidelity (it possesses no $3^{\prime}-5^{\prime}$ exonuclease activity) [228]. Importantly, the high mutability was also shown to constitute an adaptive advantage: when Yeiser et al. co-incubated umu-deficient and wildtype E. coli, the wildtype always outgrew and ultimately eliminated the mutant [230]. The multiple copies of $u m u C / D$ in the polar Octadecabacter genomes may therefore constitute less the result, and more a means of adaptation to a new environment.

The short hypothetical protein found in conjunction with both $u m u$ genes contains a domain of unknown function (DUF) 1127, and has orthologues in multiple other Roseobacter group bacteria.

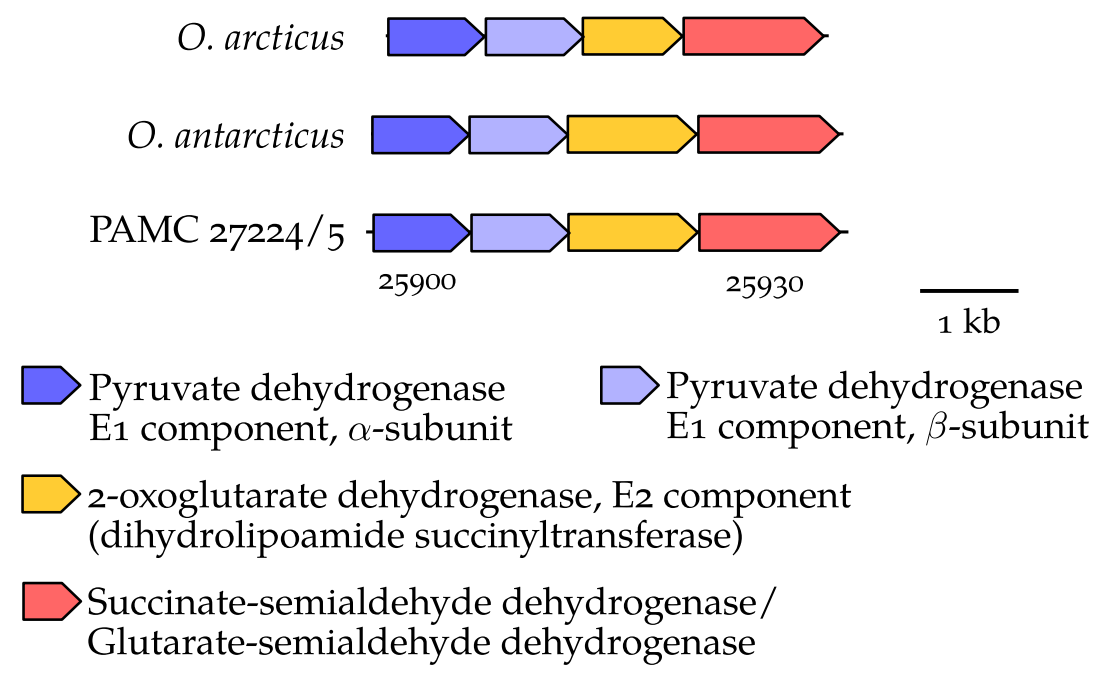

Figure 3.18: Dehydrogenase gene cluster specific to polar Octadecabacters

Four dehydrogenase genes which are involved in the TCA cycle, the central component of cellular metabolism, are specific for polar Octadecabacters (Figure 3.18). These seem to represent additional variations of common TCA cycle components, which are non-orthologous to those of the other Octadecabacters. Although redundant, the fact that the four genes are group-specific and conserved in order suggests that they are part of a specific regulatory stage in polar Octadecabacters.

According to automatic annotation of isolate PAMC 27224, two genes code for the E1 component of pyruvate dehydrogenase, although these are usually difficult to distinguish from the respective subunits of ace- 
toin dehydrogenase. Both enzyme complexes form Ac-CoA, the former from pyruvate, thereby linking glycolysis with the TCA cycle, and the latter from acetoin [231].

2-Oxoglutarate $(=\alpha$-ketoglutarate $(\alpha-\mathrm{KG}))$ dehydrogenase forms succinyl-CoA from $\alpha$-KG.

The three enzyme complexes are structurally related. Each consists of three components (named E1-3), the last of which is freely shared between all [231]. However, none of the other components were found as orthologues specific for the polar Octadecabacters.

Succinate-semialdehyde dehydrogenase produces succinate from its semialdehyde, which in turn derives from, e.g. putrescine degradation [215].

The TCA cycle is a central component of cellular metabolism, both providing reducing equivalents for oxidative phosphorylation, as well as contributing important precursor molecules to biosynthesis, among other functions [232]. Flux models of E. coli predict that this central pathway, along with the amino acid and purine synthesis pathways and a few others, dominates metabolic flux in the cell at most times, a network which is termed the high-flux backbone (HFB) [233]. It is this HFB through which the cell reacts to changes in nutrient availability, by changing the flux through individual reactions, and (de-)activating relevant auxiliary pathways [233]. Consequently, concomitant expression of the four dehydrogenase genes, induced under specific circumstances, may serve metabolic steering in the polar group, and may be part of a cellular state or strategy, which is not encountered in the other Octadecabacters. For example, pyruvate/acetoin dehydrogenase would increase flux through the TCA cycle by providing Ac-CoA, $\alpha-\mathrm{KG}$ dehydrogenase would steer flux towards succinate (away from glutamate synthesis), which would also be provided by succinate-semialdehyde dehydrogenase. This configuration would consequently either lead to increased generation of reducing equivalents and ATP through the TCA cycle, or feed into biosynthesis pathways, which consume succinyl-CoA, most importantly cobalamin and other tetrapyrroles [234].

The polar-specific genes, which are not part of consecutive regions, generally mirror the adaptations described above, i.e. they are involved in the cell's response to oxidative stress, broaden the range of usable substrates, or convey similar functions. For example, in addition to NfuA, two further group-specific enzymes are involved in sulphur biochemistry: one protein of the cysteine desulphurase-family (octa_09620), and a thiol-disulphide oxidoreductase of the DCC family (octa_14550). The former transfers sulphur from cysteine to a range of possible targets, and may be involved in biosynthetic processes of thiamine, biotin, FeS-clusters, thionucleosides, and many other compounds [235]. The latter class of enzymes manages the redox balance of thiol-disulphides of the intra- and extracellular spaces [236]. In their description of the DCC family, Ginalski et al. noted this family's non- 
universal taxonomic distribution, and high evolutionary distance from the related thioredoxin-like families, which they took as an indicator of a specific, however still undetermined, function [237].

Besides coping with an increased presence of reactive oxygen species, bacteria may adapt to alleviate the effects of higher oxygen solubility at low temperatures by consuming more of it in enzymatic reactions [39]. Beside possessing, on average, slightly more genes coding for (oxygen consuming) dioxygenases than the temperate clade members, polar Octadecabacters also possess group-specific enzymes, which convey this function. One of these is the taurine dioxygenase TauD (octa_12020), which cleaves taurine to sulphite and aminoacetaldehyde under consumption of molecular oxygen [238]. They further possess a groupspecific glycine/D-amino acid oxidase (octa_06740), which partakes in the degradation of amino acids through oxidative deamination [239].

As mentioned earlier, the polar Octadecabacters may frequently encounter situations of osmotic stress. Fittingly, they possess a groupspecific cation $/ \mathrm{H}^{+}$antiporter of the CPA1 family (octa_12300), which is known to confer increased salt tolerance through increased export of cations from the cell [240]. This type of anitporter was also shown to be present in the genomes of other cold-adapted bacteria [39].

Regarding substrate utilisation, multiple individual components of uptake systems for, e. g. phosphate, ribose, or simple sugars were found specific for the polar group, in addition to those contained in the loci described above. Furthermore, a group-specific 4- $\alpha$-glucanotransferase (octa_1068o) may avail them maltose [241], or storage polysaccharides such as glycogen [242] for degradation. These genes, in addition to the examples discussed throughout this section, hint towards patterns of nutrient availability which differ from the temperate Octadecabacter clade members. A concomitant difference in co-factor synthesis and utilisation was already indicated by the polar group-specific $m t n A$ gene, and, in addition, a pyridoxamine $5^{\prime}$-phosphate oxidase gene is specific for polar Octadecabacters (octa_41850). This enzyme catalyses the last step in pyridoxal-phosphate (vitamin $\mathrm{B}_{6}$ ) synthesis [243].

Finally, a copy of the small ribosomal subunit protein $S_{21}$ (gene name rpsU2, octa_12240), specific to polar Octadecabacters, is of note: expression of this gene was found to increase drastically at lower temperatures in the Cyanobacterium Anabaena variabilis [32], and it is part of a cold shock-responsive operon found in Sinorhizobium meliloti [33]. Consequently, it seems to be directly involved in alleviating the effects of lower temperatures by stabilising the ribosomal complex, as opposed to countering indirect effects, such as increased oxidative stress.

\subsubsection{Genes specific to temperate Octadecabacter isolates}

The majority of genes specific to both O. ascidiaceicola and O. temperatus could not be assigned a specific function, as the products of 
43 out of 68 specific genes are annotated as hypothetical proteins (compare Table A.3, p. 138). This indicates that the biochemical or metabolic features, which define this group, are generally poorly characterised. In addition, most of the functions assigned to group-specific genes are not exclusive to these two members. These genes constitute nonorthologous versions of functions, which are also part of the Octadecabacter core-genome (unlike with most of the polar Octadecabacterspecific genes discussed in the previous section).

Among the genes with an assigned function, those involved in stress resistance dominate numerically. However, none of them are organised in group-specific loci, as found in polar Octadecabacters. Two genes belong to the universal stress protein (Usp) A-family (COG 0589), namely the stress response protein NhaX (oasc_04570, a gene exclusively present in this group), and Usp F (oasc_20280). Its members are usually involved in the response to a wide range of stress signals, such as starvation, oxidative stress, or DNA damage [244]. For dealing with the latter, both Octadecabacters possess a group-specific copy each of DNA polymerase IV (oasc_15410), and a 3-methyl-adenine DNA glycosylase (oasc_25850). Like polar Octadecabacters, this group therefore seems to possess increased DNA repair capability, although both functions are also present in the Octadecabacter core-genome. The tellurite resistance protein TerB (oasc_128oo) is specifically required to increase tellurite resistance, but is also likely involved in integrating more diverse stress signals [245]. Taken together, these group-specific genes hint towards differences in the general stress response network of this group. This may correlate with a group-specific orthologue of a toluene efflux pump precursor (TtgF, oasc_10420), as well as a poly- $\beta-1,6-\mathrm{N}^{-}$ acetyl-D-glucosamine synthase (oasc_28310), and exopolysaccharide synthesis ExoD (oasc_02050), which are both involved in biofilm formation [246, 247]. Organic solvents as stress agents, and biofilm formation in response to stress signals may be of higher relevance for these particular strains, and could be integrated into the general stress response, among others, by some of the genes described above. Similar functionalities are also encoded in the Octadecabacter core-genome, and a detailed account of the environmental integration of this group would require experimental examination (e.g. via transcriptomic analyses). A further interesting characteristic is the presence of a group-specific spermidine synthase gene (oasc_20220), as this polyamine is involved in the protection of DNA against oxidative stress, and biofilm formation and surface-associated motility [248, 249]. A non-orthologous form of this enzyme is only present in the shell-genome of isolate $\mathrm{NH}_{9}-\mathrm{P}_{7}$.

Only few group-specific functions are associated with metabolism. They include an $\alpha$-ketoglutaric semialdehyde dehydrogenase (oasc 21950), which catalyses the last step in L-arabinose, and L-proline degradation, among other substrates [250]. Thus, expansion of the substrate range beyond that encoded in the Octadecabacter core-genome is not 
as pronounced in this group as it is in polar Octadecabacters, based on genes with functional annotation.

Notably, none of the functionally characterised, group-specific OGs are associated with genetic mobility (such as transposases or insertion sequences (ISs)), underlining that the abundance of such functions is a particular characteristic of polar Octadecabacters.

\subsubsection{Genes specific to Pseudooctadecabacter-related genomes}

The most striking characteristic of group III, formed by P. jejudonensis and isolate $\mathrm{NH}_{9}-\mathrm{P}_{7}$, is their genetic ability to perform aerobic anoxygenic photosynthesis (AAP), which constitutes 39 out of 78 groupspecific genes (Table A.4, p. 140). Photochemical reaction centres generally seem to increase metabolic efficiency, i. e. they maximise the amount of biomass an organism can produce from its available nutrients [251]. Their activity generates radicals, and is therefore a source of oxidative stress [252]. Nonetheless, the former aspect seems to be more important in the ecology of the two members, underlined by the presence of a group-specific starvation-inducible DNA-binding protein (OJEJ_33070).

Two out of nine Octadecabacter clade strains being theoretically able to perform AAP fits the larger context of the Roseobacter group, for which 31 out of 116 strains possess the relevant genes.

Interestingly, despite a higher potential load of autogenic oxidative stress induced by performing AAP, the group-specific genetic equipment relating to the cellular stress response is small compared to groups I and II. For example, specific functions pertaining to DNA damage repair, or the protection of sulphur species are absent. Both strains possess a specific copy of a CspA-family $\beta$-ribbon cold-shock protein (OJEJ_11070). This family acts to some extend in the transcriptional regulation of genes involved in the cold-shock response, but, more importantly, likely halts translation upon cold-shock until the organism can adapt its metabolic network to the new conditions [30]. As such, this family is also present in all other Octadecabacter clade genomes, but seems to play a larger role in the ecology and metabolic regulation of $P$. jejudonensis and isolate $\mathrm{NH}_{9}-\mathrm{P}_{7}$.

As with group II, specific genes of group III are not organised in functional loci (except for AAP-genes), and do not contain transposases or other indicators of genetic mobility.

\subsection{FUNCTIONAL GENE CONTENT EVOLUTION}

Lineage-specific parameter values of the birth-and-death model are depicted in Figure 3.19 for an overview of each parameter's relative importance at a given node in the Octadecabacter clade. Note that all parameters are normalised to $\mu$, which therefore equals 1 in all charts. 
The plot in the upper right corner shows the correlation between the edge length parameter t estimated by CounT, and the respective edge's length in the reference tree (Figure 3.2), subdivided into internal and terminal branches.

Changes in COGs at the polar Octadecabacters' latest common ancestor (LCA) are given in Table 3.5. Three polar Loktanella isolates were included in the birth-and-death model, which enabled a comparison of COG changes along their and the polar Octadecabacters' lineages. Only COG changes at internal, i.e. ancestral nodes were considered to that end, and are listed in Tables A.7 to A.11 (see Figure A.8 on p. 172 for an overview). The ancestor of the two temperate species $O$. temperatus and $O$. ascidiaceicola was included as a non-polar comparison group. In general, ancestors of polar strains do not show higher similarity to each other than to the temperate ancestor. This situation remains unchanged when gains and losses at their respective leaf nodes are included in the comparison. Two COGs were specifically newly gained at the ancestral lineages of all polar isolates: COG 1484 (DNA replication protein DnaC), and COG 2608 (Copper chaperone CopZ). Conversely, only one COG shows similar behaviour between the temperate and more than one polar ancestor: COG 0848 (Biopolymer transport protein ExbD).

General pathways affected by the COG content changes listed in Table 3.5 will be described in the following sections.

Table 3.5: Changes in COGs at the polar Octadecabacters' LCA, as predicted by Count. Only COGs with a probability of change $p>0.5$ at this node are listed. The approximate probability ratios are given on the right, with green and red indicating gain and loss, respectively. Relevant probabilities are gain $(G)$ : expansion (E) : neutral (N, no change), and loss $(\mathrm{L})$ : reduction $(\mathrm{R})$ : neutral $(\mathrm{N})$. A box indicates the highest probability, and is filled if it surpassed the secondhighest by at least $50 \%$. The rightmost column gives the orders of magnitude between the highest and lowest ratio (the latter is always 1). COGs are sorted by category, with the respective category code given on the left. COGs assigned to more than one category are listed multiple times.

\begin{tabular}{|c|c|c|c|c|c|}
\hline & COG ID & DESCRIPTION & & $\mathrm{N} / \mathrm{L}$ & $: N$ \\
\hline \multirow{6}{*}{$\mathrm{C}$} & 0578 & $\begin{array}{l}\text { Glycerol-3-phosphate } \\
\text { dehydrogenase }\end{array}$ & 1 & 75 & $7310^{3}$ \\
\hline & 1071 & $\begin{array}{l}\text { TPP-dependent pyruvate or } \\
\text { acetoin dehydrogenase } \\
\text { subunit } \alpha\end{array}$ & 1 & 51 & $2710^{4}$ \\
\hline & 1359 & Quinol monooxygenase YgiN & 319 & 1 & 319 \\
\hline & 1454 & Alcohol dehydrogenase, class IV & 5 & 1 & 2 \\
\hline & 3794 & Plastocyanin & 3 & 4 & 1 \\
\hline & $55^{24}$ & Bacteriorhodopsin & 173 & 1 & $15 \quad 10^{3}$ \\
\hline
\end{tabular}


Table 3.5: continued

\begin{tabular}{|c|c|c|c|c|c|c|}
\hline & COG ID & DESCRIPTION & G:F & $\mathrm{N} / \mathrm{L}$ & $\mathrm{R}: \mathrm{N}$ & \\
\hline \multirow{11}{*}{$\mathrm{E}$} & 0182 & $\begin{array}{l}\text { Methylthioribose-1-phosphate } \\
\text { isomerase (methionine salvage } \\
\text { pathway), a paralog of eIF-2B } \\
\alpha \text { subunit }\end{array}$ & 105 & 1 & 19 & $10^{3}$ \\
\hline & 0263 & Glutamate 5-kinase & 1 & 4 & 1 & $10^{5}$ \\
\hline & 0620 & $\begin{array}{l}\text { Methionine synthase II } \\
\text { (cobalamin-independent) }\end{array}$ & 497 & 1 & 200 & \\
\hline & 1231 & Monoamine oxidase & 1 & 11 & 5 & \\
\hline & 2113 & $\begin{array}{l}\text { ABC-type proline/glycine } \\
\text { betaine transport system, } \\
\text { periplasmic component }\end{array}$ & 1 & 55 & 40 & $10^{4}$ \\
\hline & 2986 & Histidine ammonia-lyase & 39 & 1 & 14 & $10^{2}$ \\
\hline & 2987 & Urocanate hydratase & 44 & 1 & 15 & $10^{2}$ \\
\hline & 3741 & $\begin{array}{l}\mathrm{N} \text {-formylglutamate } \\
\text { amidohydrolase }\end{array}$ & 1 & 50 & 17 & $10^{4}$ \\
\hline & 3931 & $\begin{array}{l}\text { Predicted } \mathrm{N} \text {-formylglutamate } \\
\text { amidohydrolase }\end{array}$ & 1 & 36 & 22 & $10^{3}$ \\
\hline & 4175 & $\begin{array}{l}\text { ABC-type proline/glycine } \\
\text { betaine transport system, } \\
\text { ATPase component }\end{array}$ & 1 & 38 & 27 & $10^{4}$ \\
\hline & 4176 & $\begin{array}{l}\text { ABC-type proline/glycine } \\
\text { betaine transport system, } \\
\text { permease component }\end{array}$ & 1 & 38 & 27 & $10^{4}$ \\
\hline \multirow[t]{2}{*}{$\mathrm{F}$} & 0737 & $\begin{array}{l}2^{\prime}, 3^{\prime} \text {-cyclic-nucleotide } \\
2^{\prime} \text {-phosphodiesterase } / 5^{\prime} \text { - or } \\
\text { 3'-nucleotidase, } 5^{\prime} \text {-nucleotidase } \\
\text { family }\end{array}$ & 1 & 4 & 3 & $10^{3}$ \\
\hline & 1051 & $\begin{array}{l}\text { ADP-ribose pyrophosphatase } \\
\text { YjhB, NUDIX family }\end{array}$ & 1 & 30 & 9 & $10^{3}$ \\
\hline \multirow{5}{*}{ G } & 0058 & Glucan phosphorylase & $7^{8}$ & 1 & 7 & $10^{3}$ \\
\hline & 1640 & 4- $\alpha$-glucanotransferase & 78 & 1 & 7 & $10^{3}$ \\
\hline & 2376 & Dihydroxyacetone kinase & 745 & 1 & 465 & \\
\hline & 0351 & $\begin{array}{l}\text { Hydroxymethylpyrimidine / } \\
\text { phosphomethylpyrimidine } \\
\text { kinase }\end{array}$ & 25 & 1 & 1 & \\
\hline & $035^{2}$ & $\begin{array}{l}\text { Thiamine monophosphate } \\
\text { synthase }\end{array}$ & 1 & 95 & & $10^{2}$ \\
\hline
\end{tabular}


Table 3.5: continued

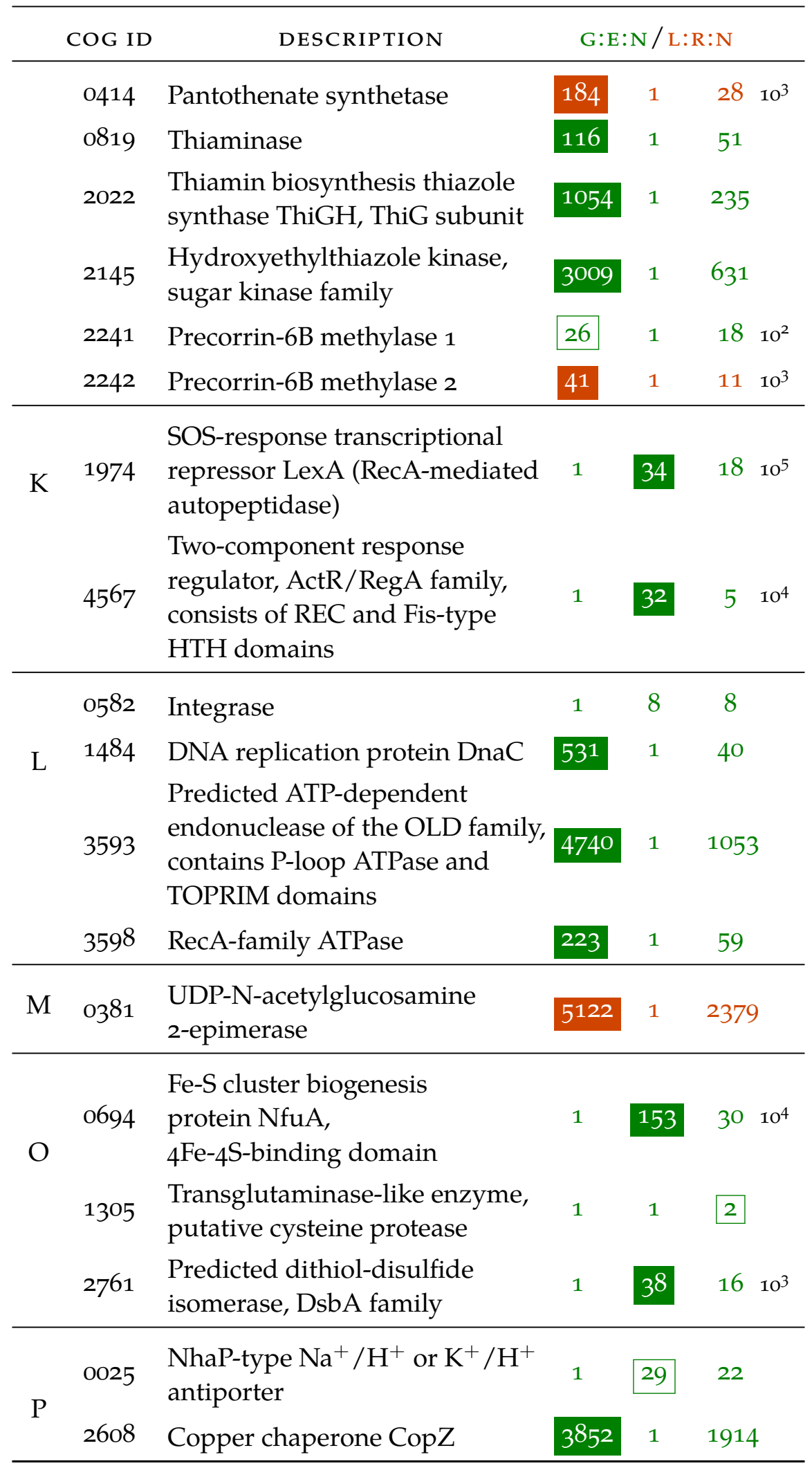


Table 3.5: continued

\begin{tabular}{|c|c|c|c|c|c|c|}
\hline & COG ID & DESCRIPTION & $\mathrm{G}: \mathrm{E}$ & $\mathrm{N} / \mathrm{L}:$ & $\mathrm{R}: \mathrm{N}$ & \\
\hline & 4638 & $\begin{array}{l}\text { Phenylpropionate dioxygenase } \\
\text { or related ring-hydroxylating } \\
\text { dioxygenase, large terminal } \\
\text { subunit }\end{array}$ & 1 & 15 & 101 & $10^{3}$ \\
\hline Q & 1228 & $\begin{array}{l}\text { Imidazolonepropionase or } \\
\text { related amidohydrolase }\end{array}$ & 107 & 1 & 67 & \\
\hline \multirow{8}{*}{$\mathrm{R}$} & 0385 & $\begin{array}{l}\text { Predicted } \mathrm{Na}^{+} \text {-dependent } \\
\text { transporter }\end{array}$ & 652 & 1 & 644 & \\
\hline & 2321 & Predicted metalloprotease & 32 & 1 & 241 & $10^{2}$ \\
\hline & 2910 & Putative NADH-flavin reductase & 1507 & 1 & 569 & \\
\hline & 3380 & $\begin{array}{l}\text { Predicted NAD/FAD-dependent } \\
\text { oxidoreductase }\end{array}$ & 105 & 1 & 341 & $10^{2}$ \\
\hline & 3450 & $\begin{array}{l}\text { Predicted enzyme of the } \\
\text { cupin superfamily }\end{array}$ & 932 & 1 & 87 & \\
\hline & 3607 & $\begin{array}{l}\text { Predicted lactoylglutathione } \\
\text { lyase }\end{array}$ & 61 & 1 & 411 & $10^{2}$ \\
\hline & 4122 & $\begin{array}{l}\text { Predicted } \\
\text { O-methyltransferase YrrM }\end{array}$ & 41 & 1 & 31 & $10^{3}$ \\
\hline & 4638 & $\begin{array}{l}\text { Phenylpropionate dioxygenase } \\
\text { or related ring-hydroxylating } \\
\text { dioxygenase, large terminal } \\
\text { subunit }\end{array}$ & 1 & 150 & 98 & $10^{2}$ \\
\hline \multirow{6}{*}{ S } & 0398 & $\begin{array}{l}\text { Uncharacterized membrane } \\
\text { protein YdjX, TVP } 38 / T M E M 64 \\
\text { family, SNARE-associated } \\
\text { domain }\end{array}$ & 1 & 88 & 331 & \\
\hline & 1357 & $\begin{array}{l}\text { Uncharacterized protein } \mathrm{YjbI}_{\mathrm{j}} \\
\text { contains pentapeptide repeats }\end{array}$ & 72 & 1 & 32 & \\
\hline & 2833 & $\begin{array}{l}\text { Uncharacterized conserved } \\
\text { protein, contains ferritin-like } \\
\text { DUF455 domain }\end{array}$ & 24 & 1 & 41 & $10^{3}$ \\
\hline & 2841 & $\begin{array}{l}\text { Uncharacterized conserved } \\
\text { protein } \mathrm{YdcH}, \mathrm{DUF}_{4} 65 \text { family }\end{array}$ & 57 & 1 & 181 & $10^{3}$ \\
\hline & 4067 & $\begin{array}{l}\text { Uncharacterized conserved } \\
\text { protein }\end{array}$ & 75 & 1 & 101 & $10^{3}$ \\
\hline & 5345 & Uncharacterized protein & 303 & 1 & 101 & $10^{3}$ \\
\hline
\end{tabular}


Table 3.5: continued

\begin{tabular}{|c|c|c|c|c|c|}
\hline & COG ID & DESCRIPTION & $\mathrm{G}: \mathrm{E}$ & N/L:I & $\mathrm{R}: \mathrm{N}$ \\
\hline \multirow{4}{*}{$\mathrm{T}$} & 1409 & $\begin{array}{l}3^{\prime}, 5^{\prime} \text {-cyclic AMP } \\
\text { phosphodiesterase CpdA }\end{array}$ & 4 & 4 & 1 \\
\hline & 1974 & $\begin{array}{l}\text { SOS-response transcriptional } \\
\text { repressor LexA (RecA-mediated } \\
\text { autopeptidase) }\end{array}$ & 1 & 340 & $17610^{4}$ \\
\hline & 4567 & $\begin{array}{l}\text { Two-component response } \\
\text { regulator, ActR/RegA family, } \\
\text { consists of REC and Fis-type } \\
\text { HTH domains }\end{array}$ & 1 & 320 & $5410^{3}$ \\
\hline & 5524 & Bacteriorhodopsin & 173 & 1 & $1510^{3}$ \\
\hline $\mathrm{U}$ & 3505 & $\begin{array}{l}\text { Type IV secretory pathway, } \\
\text { VirD4 component, } \\
\text { TraG/TraD family ATPase }\end{array}$ & 1196 & 1 & 360 \\
\hline \multirow[t]{3}{*}{ V } & O737 & $\begin{array}{l}\text { 2',3'-cyclic-nucleotide } \\
2^{\prime} \text {-phosphodiesterase / } 5^{\prime} \text { - or } \\
3^{\prime} \text {-nucleotidase, } 5^{\prime} \text {-nucleotidase } \\
\text { family }\end{array}$ & 1 & 4113 & 3321 \\
\hline & 1787 & $\begin{array}{l}\text { Endonuclease, } \\
\text { HJR/Mrr/RecB family }\end{array}$ & 2075 & 1 & 1765 \\
\hline & 2746 & $\begin{array}{l}\text { Aminoglycoside } \\
\mathrm{N}_{3} \text {-acetyltransferase }\end{array}$ & 462 & 1 & $3710^{3}$ \\
\hline \multirow{6}{*}{$X$} & 0582 & Integrase & 1 & 8 & 8 \\
\hline & 1943 & $\begin{array}{l}\text { REP element-mobilizing } \\
\text { transposase RayT }\end{array}$ & 2 & 1 & 1 \\
\hline & 2826 & $\begin{array}{l}\text { Transposase and inactivated } \\
\text { derivatives, IS3o family }\end{array}$ & 237 & 22 & 1 \\
\hline & 3415 & Transposase & 970 & 1 & 619 \\
\hline & 3547 & Transposase & 18 & 6 & 1 \\
\hline & 5433 & $\begin{array}{l}\text { Predicted transposase } \\
\text { YbfD/YdcC associated with } \\
\text { H repeats }\end{array}$ & 1841 & 1 & 983 \\
\hline
\end{tabular}

\subsubsection{Thiamine metabolism}

All sequenced Octadecabacter clade members possess the genes necessary for thiamine uptake (via the ABC-transporter ThiBPQ) and subsequent conversion to thiamine di- and triphosphate (via the thiamine 


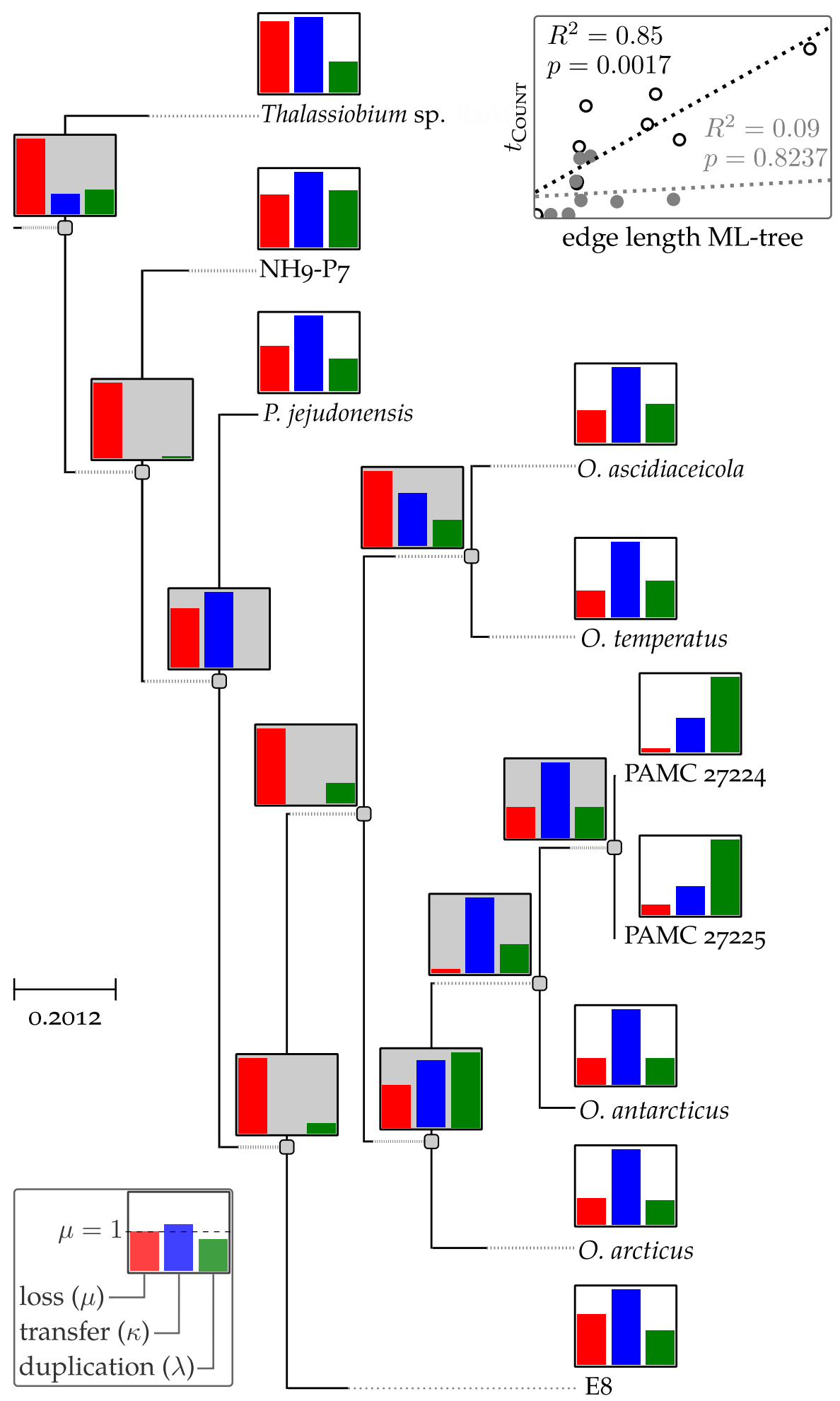

Figure 3.19: Lineage-specific components of transfer $(\kappa)$, loss $(\mu)$, duplication $(\lambda)$, and edge length ( $t$ ) parameters, as estimated by CounT (Section 2.9). Tree presents $t$ directly (but disregard dotted lines), while the other parameters are provided in bar charts. All parameters (including $t$ ) are normalised to $\mu$, which therefore equals 1 in all charts. Charts at internal nodes are filled with grey background. Plot shows relation between $t$ and edge length in the ML-tree for internal (grey) and terminal nodes (hollow circles). 
pyrophosphatase ThiN). In addition, the polar Octadecabacters, as well as O. ascidiaceicola, can synthesise thiamine de novo: COGs 0351, 0352, and 2022 constitute three genes in thiamine biosynthesis (thiD, E, and $G$, respectively), to which the phylogenetic birth-and-death model assigns high likelihoods of gain (thiD, G), or expansion (thiE) at the polar Octadecabacters' ancestral node (see Table 3.5, category H). There are multiple other genes required for thiamine synthesis (summarised well by Jurgenson, Begley, and Ealick [253]), all of which are present in the five genomes. The model assigns a higher probability to the independent acquisition of the respective genes by O. ascidiaceicola, than to them being present in a common ancestor of (and subsequent vertical heredity to) the polar Octadecabacters and O. ascidiaceicola (and their loss in O. temperatus). Interestingly, the five genomes also contain a single copy of a THI5-like gene. These are known from yeasts, in which they synthesise hydroxymethyl pyrimidine (HMP), one of the two thiamine precursors, from pyridoxin and histidine [254]. Usually, in prokaryotes ThiC, which is also present in all five Octadecabacter clade genomes, synthesises this compound from 5 -aminoimidazole ribotide, an intermediate in purine biosynthesis [253]. In contrast to $\mathrm{THI}_{5}$, ThiC consumes S-adenosylmethionine (SAM) (in form of a $5^{\prime}$ deoxyadenosyl radical), and requires a $4 \mathrm{Fe}-4 \mathrm{~S}$ cluster as co-factor.

COGs 0819 (TenA) and 2145 (ThiM) encode enzymes involved in thiamine salvage [253], the latter being exclusive to the polar members. Of the two, TenA cleaves the unphosphorylated form of thiamine and seems to function in recycling of the HMP moiety [255], while ThiM functions in thiazole-salvage [253].

Generally, thiamine, like the other B vitamins, is a co-factor often exchanged between microbes in mutualistic relationships [256]. Presence of its biosynthetic pathway in the polar Octadecabacters and $O$. ascidiaceicola therefore reflects differences in these relationships in their respective environments compared to the other clade members. Thiamine is produced by the majority of marine prokaryotes, while many marine eukaryotes are auxotrophic for it [256]. The latter comprise a large fraction of the active microbes in sea ice [41], and consequently, polar Octadecabacters may take up the role of thiamine providers in exchange for, e.g. photosynthates, a relationship which was demonstrated for other Roseobacter group bacteria as well [257]. An alternative explanation may be that the Octadecabacters preferentially take up thiamine from the environment, and only synthesise it during phases of low nutrient availability. Polar Octadecabacters are, for example, confronted with such situations when the sea ice melts, and they are expelled to the open ocean. Their need to efficiently recycle nutrients in order to survive is further signified by the fact that they possess genes for thiamine degradation which are absent in the rest of the clade. 


\subsubsection{Tetrapyrrole biosynthesis}

All sequenced Octadecabacter clade genomes encode genes for the synthesis of the most common tetrapyrroles heme, siroheme, and cobalamin. The common precursor $\delta$-aminolevulinic acid is synthesised from glycine and succinyl-CoA by the gene product of hemA in the C4-pathway, which is prevalent in $\alpha$-Proteobacteria [234]. A notable exception exists in the two members $P$. jejudonensis and isolate $\mathrm{NH}_{9}-\mathrm{P}_{7}$, which are also genetically equipped to synthesise bacteriochlorophyll and perform AAP (see Section 3.4.3).

Orthologues to one of the many methyltransferases in cobalamin biosynthesis, precorrin-6B methylase ( $c o b L)$, were assigned to different COGs in different Octadecabacters, and the phylogenetic birth-anddeath model predicted their respective gain and loss at the polar Octadecabacters' LCA (COGs 2241 and 2242 in Table 3.5, category H). Notably, both COGs were registered as orthologues in the present analysis. Nonetheless, they differ in length $(\approx 240$ AA and $\approx 200$ AA for COG 2241 and 2242, respectively) and show different alignment patterns within the COG reference organisms. The polar Octadecabacters seem to have lost the second isoform (COG 2422) in favour of the first one (COG 2421). Among the non-polar Octadecabacters, the only other occurrence of this enzyme is in isolate $\mathrm{NH}_{9}-\mathrm{P}_{7}$. Within the whole Roseobacter group, COG 2422 seems to be the more prevalent, as it is shared by 85 of the 115 analysed genomes (compared to 29 for COG 2421). No clear distinction in localisation of the two enzymes seems to exist, based on the isolation coordinates of the Roseobacter group members (Table A.1). Neither does life style (as estimated from the isolation circumstances) serve as a predictor for the isoform $\left(\chi^{2}=7.03, p=0.32\right.$ and $\chi^{2}=3.86$, $p=0.7$ for COGs 2241 and 2242, respectively).

Consequently, a slight difference seems to exist between polar and non-polar Octadecabacter clade members concerning cobalamin synthesis.

\subsubsection{Methionine metabolism}

All Octadecabacters possess the necessary genes for methionine synthesis, with both methylmethionine and methyl-tetrahydrofolate (MTHF) as methyl donors. The genes likely constitute the homocysteine-responsive met $R$ regulon specific for Rhodobacterales, as recently described by Leyn et al. [258]. In addition to the cobalamin-dependent methionine synthase $(m e t H)$, the polar Octadecabacters seem to have gained the cobalamin-independent synthase (metE, see Table 3.5). This enzyme transfers the methyl group from MTHF to homocysteine via a mechanism independent from cobalamin, albeit at a markedly lower rate [259]. All Octadecabacters are able to produce cobalamin, as described in Section 3.5.2. However, its production is costly, and the cobalamin- 
independent methionine synthase may provide an alternative for the polar Octadecabacters during phases of low nutrient availability.

A further characteristic of polar Octadecabacters is the $m t n A$ gene (coding for methylthioribose-1-phosphate isomerase; see Table A.2, and COG 0182 in Table 3.5), the product of which catalyses the second step of the methionine salvage pathway [260]. This pathway recycles methylthioadenosine (MTA), a product of SAM utilisation, to regain methionine. MTA is produced through donation of an aminopropyl group from SAM, e.g. in polyamine or $\mathrm{N}$-acetyl-homoserine lactone synthesis. The first step of the methionine salvage pathway is phosphorolysis of MTA, which is catalysed by the product of $m t n P$, present in all Octadecabacters. In this step, adenine and methylthioribose (MTR)-1phosphate are formed. The latter is then isomerised to methylthioribulose-1-phosphate by the $m t n A$-coded isomerase. Subsequent conversion to methionine happens in five consecutive steps, catalysed by a dehydratase, enolase, phosphatase, dioxygenase and transaminase [261]. Notably, almost none of the respective genes could be found in the polar Octadecabacters, neither by their annotation, nor through KO terms. Only one homologue of the enolase seems to be coded by the PAMC isolates. The enolases usually involved in this reaction belong to the haloacid dehalogenase superfamily [261]. While the polar Octadecabacters have, on average, more genes of this superfamily than the temperates, there is no enolase specific for this group. Interestingly, RuBisCO-like proteins were also found to catalyse this reaction [261], and one of the genes in O. antarcticus is annotated with this function [61]. However, it does not occur as orthologue in any other Octadecabacters. The dioxygenase is usually of the cupin-superfamily [261], and there is a high probability that one such gene was exclusively gained by the polar Octadecabacters (COG 3450 in Table 3.5, category R). A high degree of flexibility is also known for the last step of the cycle, transamination to methionine [261].

Generally, the methionine salvage pathway conserves sulphur within the cell. It is only of importance, if larger amounts of SAM are used in polyamine or homoserine lactone synthesis. The polar Octadecabacters lack polyamine synthases, and therefore likely do not produce large amounts of MTA. From the produced MTA, adenine is universally recycled in the Octadecabacters as described above, but instead of recycling the resulting MTR-1-phosphate, they likely export it. A similar behaviour was observed in E. coli [262]. In E. coli, absence of the methionine salvage pathway was attributed to the high sulphur availability in its natural environment, which may also exist for the Octadecabacters (discussed in Section 4.3.2). MTR-1-phosphate has no known immediate use for cellular biochemistry, but may serve as quorum sensor in the extra-cellular space [262]. However, the question why the polar Octadecabacters possess the $m t n A$ gene remains. 


\subsubsection{Histidine degradation}

All polar Octadecabacters, as well as isolate E8 are able to use histidine as carbon and nitrogen source. COGs 2986 and 2987, which were likely independently gained by the polar Octadecabacters' LCA (Table 3.5), are the first two steps in histidine degradation, a pathway discussed in detail in ref. [263]. Histidine ammonia-lyase (hutH) deaminates histidine, producing ammonium and urocanate. The latter is then hydrated to imidazolonepropionate (hutU, COG 2987 in Table 3.5), and further hydrolysed to formiminoglutamate by an imidazolonepropionase (HutI, COG 1228 in Table 3.5, category Q). In Octadecabacters, the $h u t F$ gene product (formiminoglutamase/formiminoglutamate deiminase) cleaves a further ammonium molecule off the formimino-group, leaving formylglutamate. In a last step, $\mathrm{N}$-formylglutamate amidohydrolase or formylase, coded for by hut $G$, cleaves formylglutamate to formate and glutamate. While a gene encoding this enzyme is present in all Octadecabacters, the polar species possess more copies, and expansion likely occurred at their LCA (see COGs 3741 and 3931 in Table 3.5). Note that the ABC-type proline/glycine betaine system, which received an expansion in the polar lineage (COGs 2113, 4175 and 4176) may as well code for histidine uptake transporter, as the two are regularly confused [263].

\subsubsection{General cellular stress response}

Genes potentially involved in the response to increased levels of oxidative, osmotic, or respiratory stress comprise a significant portion of the polar Octadecabacter-specific genes discussed in Section 3.4.1. Fittingly, similar functions were gained or expanded at the polar Octadecabacters' LCA, some of which are identical to those found group-specific (e. g. NfuA).

Among the COGs gained, some are associated with a chaperone function, such as COG 2608, which represents the copper chaperone CopZ. CopZ binds copper ions for their export, and thus prevents their exposition to the cellular environment, which would result in oxidative stress [264]. Interestingly, this COG was likely gained in the ancestral lineages of all polar isolates contained in the birth-and-death model, including polar Loktanella species (Section 3.5). In addition, a DsbAfamily dithiol-disulphide isomerase (COG 2761) likely experienced family expansion at this node. This family mediates the formation of disulphide bonds in proteins exported to the periplasm [265], and thus ensures their proper structure and function.

The Octadecabacter LCA furthermore likely acquired a quinol monooxygenase (COG 1359). This enzyme oxidises quinone-derived substrates, and therefore plays a role in maintaining the proper balance of quinone and quinol pools in the electron transport chain [266]. No- 
tably, it does not require co-factors. In addition, the functional family of plastocyanins (COG 3794) likely experienced family expansion at this point, in accordance with the fact that one such protein is specific for the polar group (annotated as pseudoazurin, Table A.2). These copper-binding proteins are usually involved in electron transfer reactions to cytochromes in diverse contexts [267]. Gain and expansion of these two functions likely serves polar Octadecabacters in oxidative or respiratory stress protection.

A possible expansion of NhaP-type cation $/ \mathrm{H}^{+}$antiporters (COG 0025) may complement this functionality, since these are usually involved in maintenance of the intracellular $\mathrm{pH}$ [268]. In combination with the polar Octadecabacters' increased capacity for proton-gradient generation (due to their possible xanthorhodopsin phototrophy), this transporter type may also simply serve as a sodium exporter, and thus function to counteract osmotic stress. In addition, glutamate kinase (COG 0263), may have likely expanded at the polar Octadecabacter LCA. This enzyme catalyses the first step in proline synthesis from glutamate [269]. Proline, as well as proline betaine are both widespread osmoprotectants [270, 271]. Additional copies of this gene may serve to compensate for its feedback-inhibition by proline itself, and thus allow for the accumulation of larger proline pools during phases of osmotic stress.

Furthermore, ADP-ribose pyrophosphatase (COG 1051) likely expanded along the polar Octadecabacters' lineage. This enzyme cleaves ADP-ribose into AMP and ribose-5-phosphate, but most of its studied homologues also show reduced activity on ADP-sugars and nicotinamide adenine dinucleotide (NAD) [272, 273]. ADP-ribose is a product of NAD turnover and has potential cytotoxic effects, most importantly through non-enzymatic protein glycation [274]. Consequently, expansion in this gene family may reduce cellular damage by this compound. In addition, this class of pyrophosphatases also contains the tellurite resistance protein $\operatorname{Trg} B$, which is a major player of tellurite resistance in E. coli and Rhodobacter capsulatus [272, 275], and in the latter was shown to also increase resistance to copper [275].

Some transcriptional regulators associated with different types of stress are specific for polar Octadecabacters (Section 3.4.1). Generally, DNA damage (induced either by UV-irradiation, or oxidative stress) induces a stress-responsive network of genes in what is referred to as the SOS-response [276]. This response is mainly regulated by two proteins: LexA acts as transcriptional repressor of SOS-response-associated genes, and, upon accumulation of DNA damage, its auto-catalytic cleavage is induced by RecA [276]. The repressor LexA experienced gene family expansion with high probability in the polar Octadecabacters' ancestral lineage (COG 1974 in Table 3.5). This indicates a tighter control of the SOS-response in polar Octadecabacters, which concurs with their altered genetic equipment dealing with DNA damage (DNA polymerase $\mathrm{V}$ ). 
In summary, the diverse stress-related functions discussed above supplement those found among the polar Octadecabacter group-specific genes.

\subsubsection{Other metabolism-related functions}

Apart from (more or less) complete metabolic pathways probably gained by the polar Octadecabacters' LCA, which were described in previous sections, some single COGs showed change at this point in the phylogeny, which are associated with individual metabolic reactions and stages. One of these is COG 1409 (cyclic adenosine monophosphate (AMP) (cAMP) phosphodiesterase CpdA), which degrades cAMP and thus regulates cellular pools of this important effector molecule. In effect, $c p d A$-overexpressing E. coli cells showed increased resistance to oxidative stress, mediated by a strong induction of rpoS expression [277]. Independent from this function, CpdA also seems to be involved in global regulation of amino acid synthesis [278].

Notably, another cyclic nucleotide phosphodiesterase (COG 0737) is among the few functions that experienced family reduction along the polar Octadecabacter lineage. This periplasmic enzyme degrades cyclic nucleotides with broad specificity [279]. As such, it is important in utilising extracellular DNA as a carbon and phosphate source [280], and may be particularly relevant in habitats with phosphate-limitation. Moreover, Trülzsch et al. observed that this enzyme mediates the ability of Yersinia enterocolitica to grow on $2^{\prime}, 3^{\prime}$-cAMP as sole carbon and energy source [281].

Polar Octadecabacter-specific genes indicated a differential utilisation of glycerol and its intermediates in this group (Section 3.4.1). In addition to gaining the group-specific DHA kinase, the polar Octadecabacter LCA likely expanded in glycerol-3-phosphate dehydrogenase (GPDH) (COG 0578) functionality. This enzyme is part of the glycerol degradation pathway found in all Octadecabacters. Its increased numbers in polar representatives may reflect higher availability of this compatible solute in their habitat, due to its production by eukaryotes upon osmotic stress [209, 282]. Notably, GPDH links the fatty acid cycle with glycolysis via its inter-conversion of DHAP and glycerol-3phosphate. Expansion of this gene family may therefore serve a regulatory function, being a further indicator that polar Octadecabacters possess more complex metabolic networks, and need to integrate more complex environmental stimuli.

Apart from glycerol, primary producers frequently secrete storage saccharides, which may be degraded and metabolised by associated prokaryotes [283]. The polar Octadecabacters' LCA likely gained the group-specific 4 - $\alpha$-glucanotransferase (COG 1640), as well as a glucan phosphorylase (COG 0058); both are enzymes for the mobilisation of these polysaccharides. 
Finally, polar Octadecabacters seem to have lost the ability to synthesise pantothenate at their LCA (loss of pantothenate synthetase PanC, COG 0414). Pantothenate is the precursor of CoA, and thus essential for cellular metabolism [284]. However, it is also produced in excess quantity and secreted by many bacteria [284], and may thus be easily taken up from the environment by pantothenate-auxotrophs.

\subsection{PARALOGUE OCCURRENCE IN OCTADECABACTER CLADE MEM- BERS}

Paralogues are derived from gene duplications within an organism, and therefore show highest BLAST-based similarity to their original gene in the same genome [285]. To test the amount of duplications present in the Octadecabacter genomes, determination of paralogous relationships was included in the orthology detection (Section 2.5).

Generally, polar Octadecabacters possess more paralogues than the temperate strains, ranging from an average of 68 in the PAMC isolates to over 560 in O. arcticus, versus 22 to 45 in the other strains (Table 3.6). Isolate $\mathrm{NH}_{9}-\mathrm{P}_{7}$ constitutes a notable exception, with over 150 paralogues in total. While most duplications in the polar type strains of $O$. arcticus and $O$. antarcticus are attributable to mobile genetic elements, i. e. transposases, integrases, and ISs, they still possess a higher average number of paralogues than the others when these cases are excluded (Table 3.6).

The relative paralogue content of polar Octadecabacters, normalised to the number of CDSs (as this group possesses on average $\approx 800$ CDSs more than the temperate strains, Table 3.1), is still high compared to the other groups. It ranges from an average of 13.8 paralogues in different OGs per 1,000 CDSs in the PAMC isolates to 31.6 in O. arcticus and $O$. antarcticus (excluding mobile genetic elements). Temperate strains possess an average of 9 paralogues in different OGs per 1,000 CDSs, with the exception of isolate $\mathrm{NH}_{9}-\mathrm{P}_{7}$ (44.2). Notably, the high fragmentation of the PAMC-isolate genomes (Table 3.1) could lead to an underestimation of their total paralogue content (see Appendix A.7, p. 203).

The majority of paralogues ( 45 to $92 \%$ ) is strain-specific, only the highly similar PAMC isolates pose an exception. There are no paralogues which are specific to the polar Octadecabacters (group I), i.e. which are present in all members of this group, and absent in all members of groups II to IV. All polar isolates do, however, possess multiple paralogues in the group-specific genome (as listed in Table A.2). In fact, the fraction of their group-specific genes being duplicated in at least one of their genomes is larger than that of the other groups.

Differences are also visible in the distribution of paralogues among the core-, cloud-, and shell-genomes defined in Section 3.3.1. While gene duplications in the type strains of $O$. arcticus and O. antarcticus predominantly affect their shell-genomes, isolate $\mathrm{NH}_{9}-\mathrm{P}_{7}$, with its similar 
Table 3.6: Number of paralogues in Octadecabacter clade genomes. Grey numbers exclude the strain's mobile elements ${ }^{a}$. Group-specific paralogues are present in all members of a group (as defined in Section 3.2), and absent in all other strains. GROUP-SPECIFIC GENES as listed in Appendix A.4. Core-, cloud-, and shell-genome boundaries as defined in Section 3.3.1.

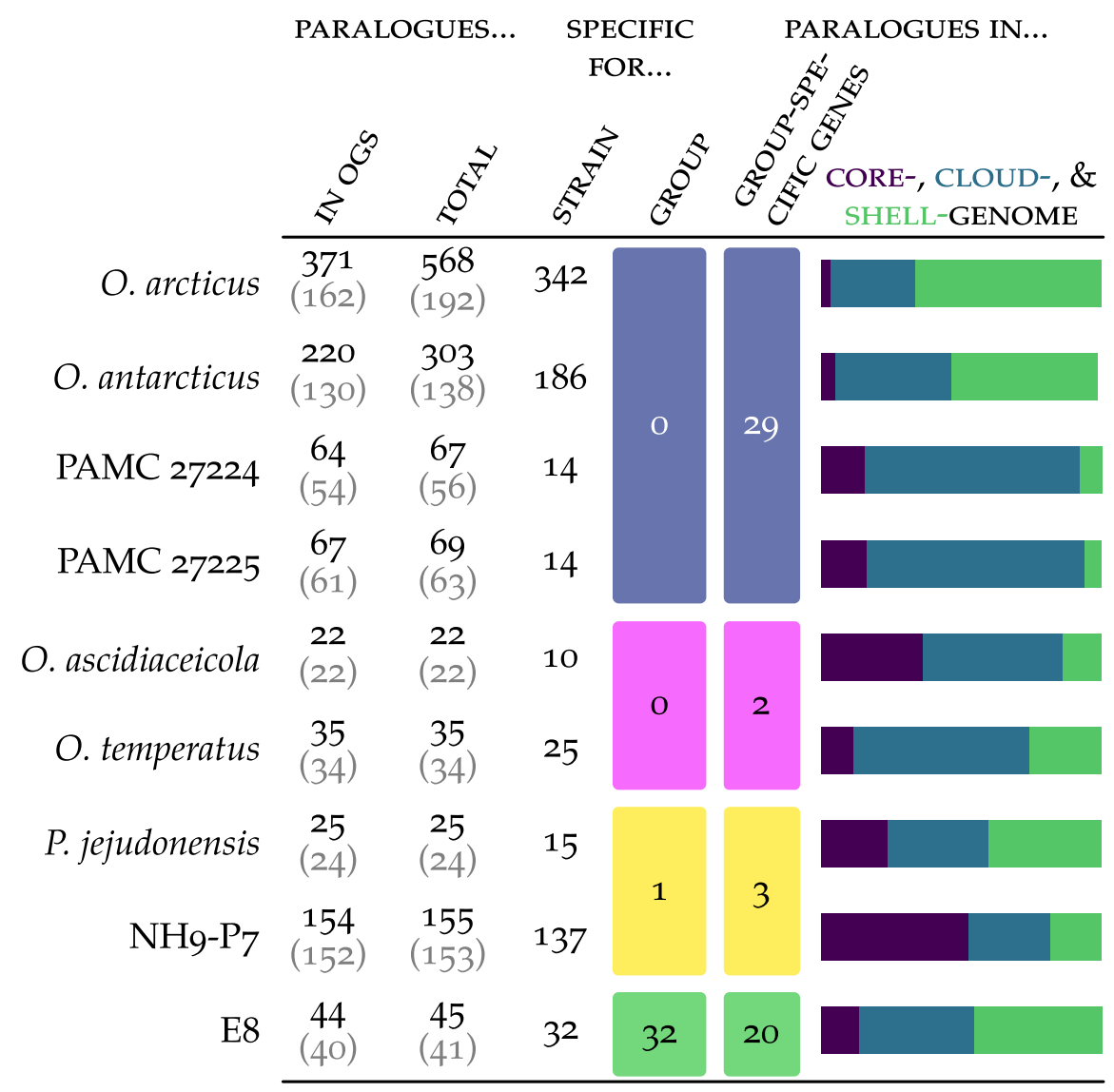

a all OGs whose annotations contained the strings "obile_element", "ransposase", "ntegrase", or "IS"

number of paralogues, shows more duplications in the Octadecabacter clade core-genome (Table 3.6).

\subsection{KEY CHARACTERISTICS IN POLAR ROSEOBACTERS}

The comparison genomes used in this study included five polar isolates, which were associated with clades other than the Octadecabacters (Table 3.7). Key figures, differing between polar and temperate Octadecabacters, were compared to these polar isolates and their associated clade members (Figure 3.20).

While polar Octadecabacters possess larger genomes with more protein-coding genes than their temperate relatives, this trend is not observable in the comparison groups. Regarding singletons, all polar isolates lie above their respective group's median (except for the highly similar PAMC isolates), but only the Octadecabacters possess the most 
Table 3.7: Isolates outside of the Octadecabacter clade, which were procured from polar environments based on their isolation coordinates (Table A.1), are listed together with the Roseobacter group bacteria with which they form common clades (according to the MLSA phylogeny, Figure A.2). These were used for comparison of key figures between polar and non-polar isolates throughout the Roseobacter group (Figure 3.20).

POLAR ISOLATES

ASS. CLADE MEMBERS

L. vestfoldensis DSM 16212, Loktanella genus-associated genomes

L. fryxellensis DSM 16213, in Table 3.2

L. salsilacus DSM 16199

Pseudophaeobacter arcticus Leisingera genus-associated genomes

DSM 23566 in Table 3.2

Sulfitobacter guttiformis Sulfitobacter genus-associated

KCTC 32187 genomes in Table 3.2, plus $S$.

donghicola JCM 14565, Sulfitobacter

sp. EE-36, Sulfitobacter sp. NAS-14.1,

Sulfitobacter sp. CB2047

within their clade. Furthermore, in three of the four analysed clades, polar isolates show the highest number of paralogues, with the polar S. guttiformis as the only exception. The differences in the Loktanella and Leisingera clades are, however, less pronounced than in the Octadecabacters.

The polar Octadecabacters' capacity for carbohydrate uptake and metabolism is likely higher than that of the temperate ones, as they possess more genes associated with COG category G. Among the other groups, only polar Loktanella species mirror this trend to a degree (2 out of 3 polar isolates). In addition, polar isolates of both clades concordantly possess more genes in functions associated with recombination and genetic mobility (COG categories L and X, respectively), whereas such a difference is lacking in the other two comparison groups. 


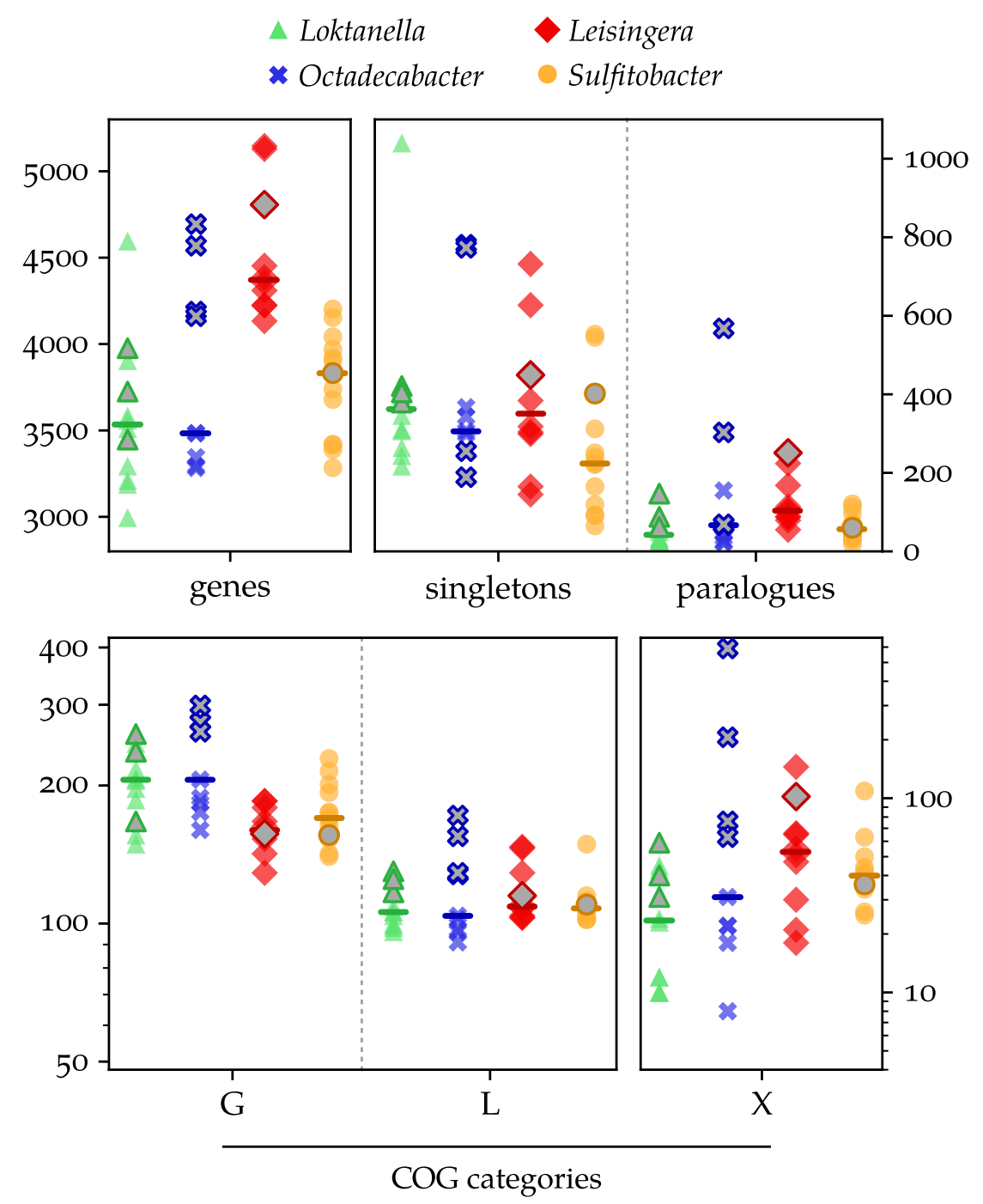

Figure 3.20: Key figures in polar roseobacters. Characteristics found to differ between polar and temperate Octadecabacters were compared to other Roseobacter group clades which harbour polar isolates. Their predominant associated genera are given on the top. Larger symbols filled with grey represent the polar isolates. Lines present median values. genes - number of proteincoding genes; singletons relating to all comparison genomes (Table 2.2) 


\subsection{PROTEIN SECONDARY STRUCTURE CONTENT}

Secondary structure content of all proteins coded in the Octadecabacter genomes was estimated using the jpred4 algorithm (Section 2.11). Pairwise euclidean distances in predicted $\alpha$-helix and $\beta$-sheet content between the Octadecabacter core proteins were used for hierarchical clustering. When both structural features are considered together, polar Octadecabacters cluster separate from the rest, and groups defined in Section 3.2 are coherent (Figure 3.21). However, this coherence is absent when both features are considered separately.

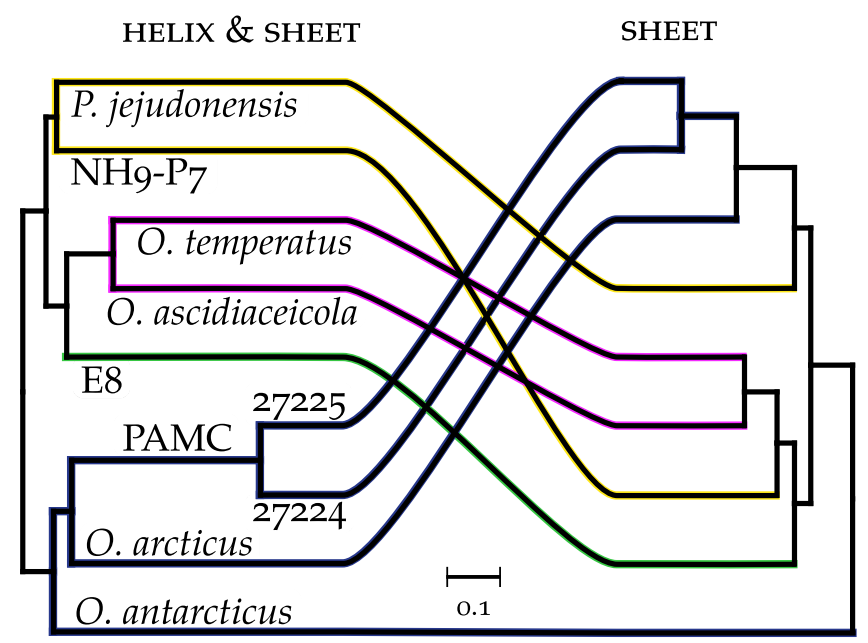

HELIX

Figure 3.21: Hierarchical clustering of Octadecabacters based on predicted protein secondary structure. Clustering was based on the pairwise euclidean distances in relative secondary structure content between all Octadecabacter clade core proteins. Both structural features ( $\beta$-sheet and $\alpha$-helix) were considered in combination (left), as well as individually (centre and right, respectively). Colours mark groups defined in Section 3.2.

Wilcoxon signed-rank tests revealed that no Octadecabacter group shows a clear and statistically significant tendency towards containing different amounts of either structural feature than the others (Figure 3.22). Solely $O$. antarcticus possesses significantly less predicted $\beta$-sheets when compared to the members of group III. A similar difference in its $\alpha$-helix content can only be observed for isolate $\mathrm{NH}_{9}-\mathrm{P}_{7}$ (and not for P. jejudonensis).

The performed $\chi^{2}$ tests detected significant $(p<0.05)$ differences in observed versus expected amino acid count for the seven compared Octadecabacter strains. However, this significance likely stems from the high number of observations, as the mean differences in amino acid frequencies between the groups are very small (on the order of $10^{-3}$, Figure 3.23). Nonetheless, polar Octadecabacters show some clear trends. For example, they possess higher portions of the hydrophobic amino acids alanine, isoleucine, and methionine (single letter codes A, I, and M, respectively). Within negatively charged amino 


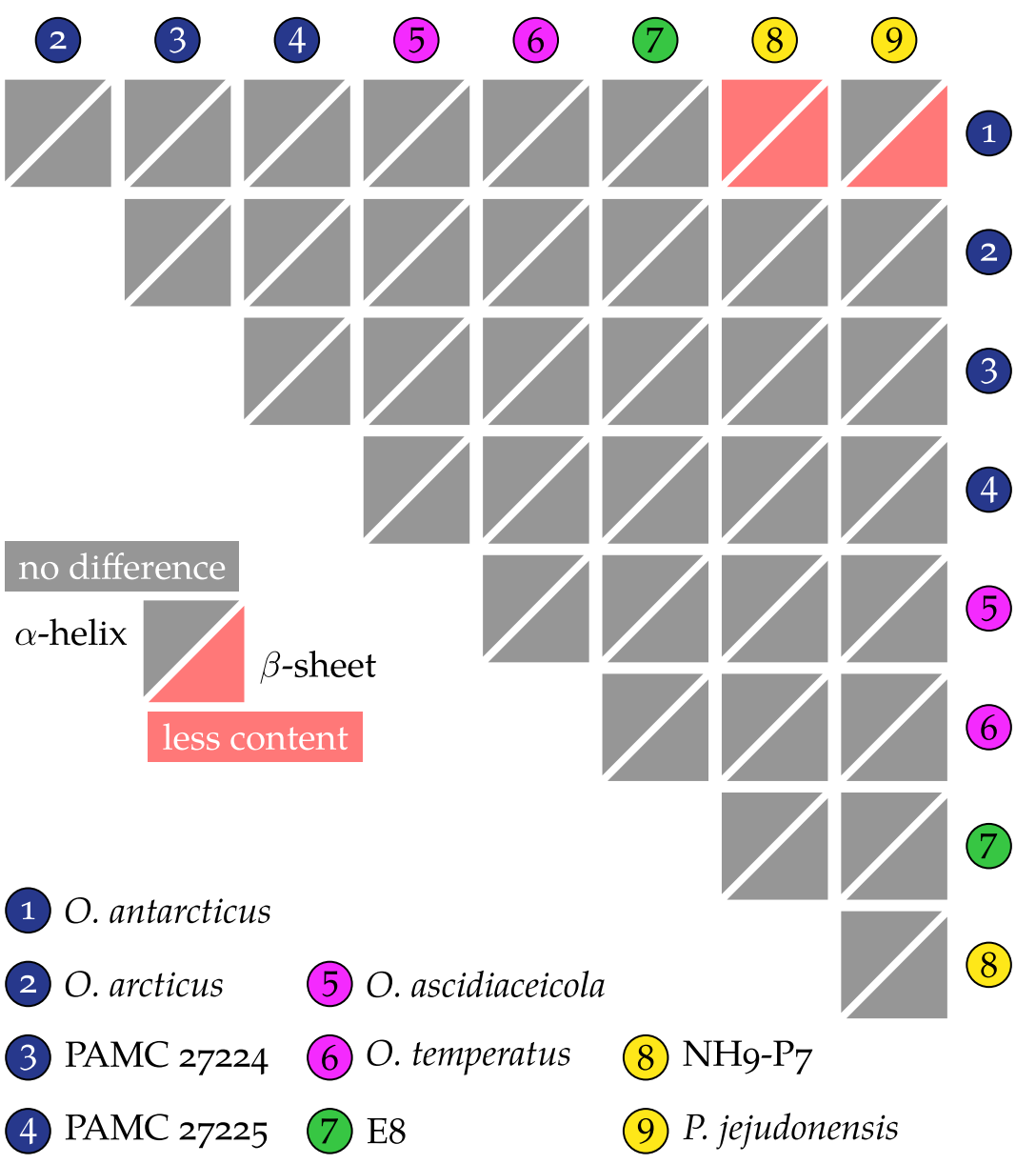

Figure 3.22: Results of Wilcoxon signed-rank tests for significant difference in secondary structure content between Octadecabacter clade members. Only O. antarcticus, when tested against the members of group III (as defined in Section 3.2), showed significantly different predicted contents under the Benjamini-Hochberg-corrected significance levels (Section 2.11). The jpred4 algorithm predicts overall less $\beta$-sheet in this strain's core-proteome than in both isolate $\mathrm{NH}_{9}-\mathrm{P}_{7}$ and $\mathrm{P}$. jejudonensis, and less $\alpha$-helix than in the former (marked in red). 


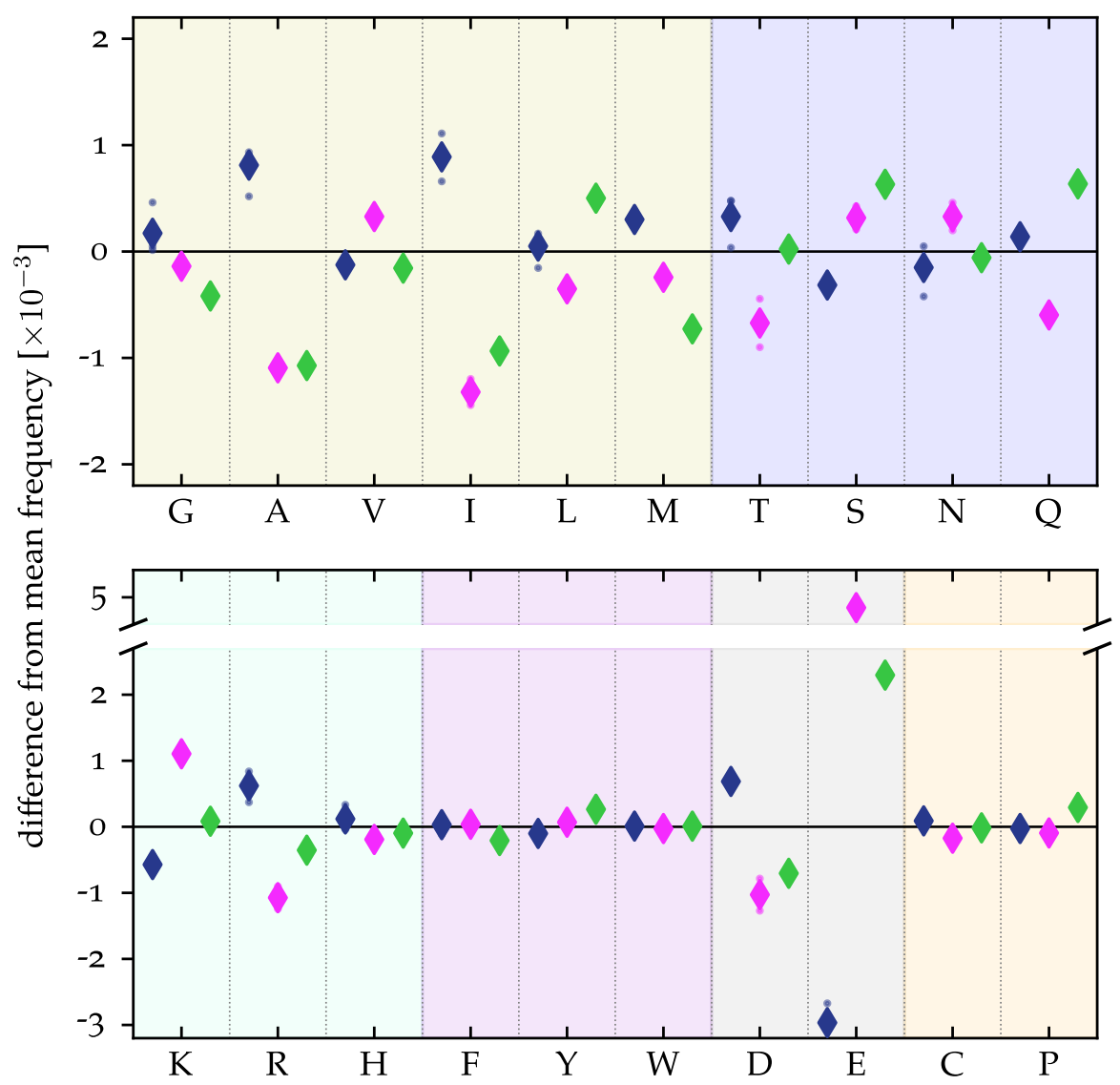

Figure 3.23: Mean amino acid frequency deviations in Octadecabacter groups. The mean amino acid frequencies in the Octadecabacter core-proteome were calculated for seven strains with similar GC-content (see Table 3.1), which included groups I, II, and IV (as defined in Section 3.2). The plot shows the difference of each group's mean to the base frequency, i. e. the mean frequency over all seven strains. Amino acids are sorted into hydrophobic (G, A, V, I, L, $\mathrm{M})$, polar uncharged $(\mathrm{T}, \mathrm{S}, \mathrm{N}, \mathrm{Q})$, aromatic $(\mathrm{F}, \mathrm{Y}, \mathrm{W})$, and negatively $(\mathrm{D}, \mathrm{E})$ and positively $(\mathrm{R}, \mathrm{H}, \mathrm{K})$ charged groups.

acids, the group seems to favour aspartic acid (D) over glutamic acid (E). Finally, the four strains contain slightly more arginine (R), and slightly less serine (S) and lysine (K) than groups II and IV (Figure 3.23).

Compared to their general frequencies, the ratio of amino acids in predicted helix versus non-helix regions shows a higher within-group variation (compare individual values in Figure 3.23 versus Figure 3.24). Distinct trends in polar Octadecabacters seem to be an increased incorporation of negatively charged amino acids (both D and E), as well as valine (V) into helices (Figure 3.24). At the same time, their predicted helices contain a lower percentage of isoleucine, methionine, asparagine $(\mathrm{N})$, and lysine. 


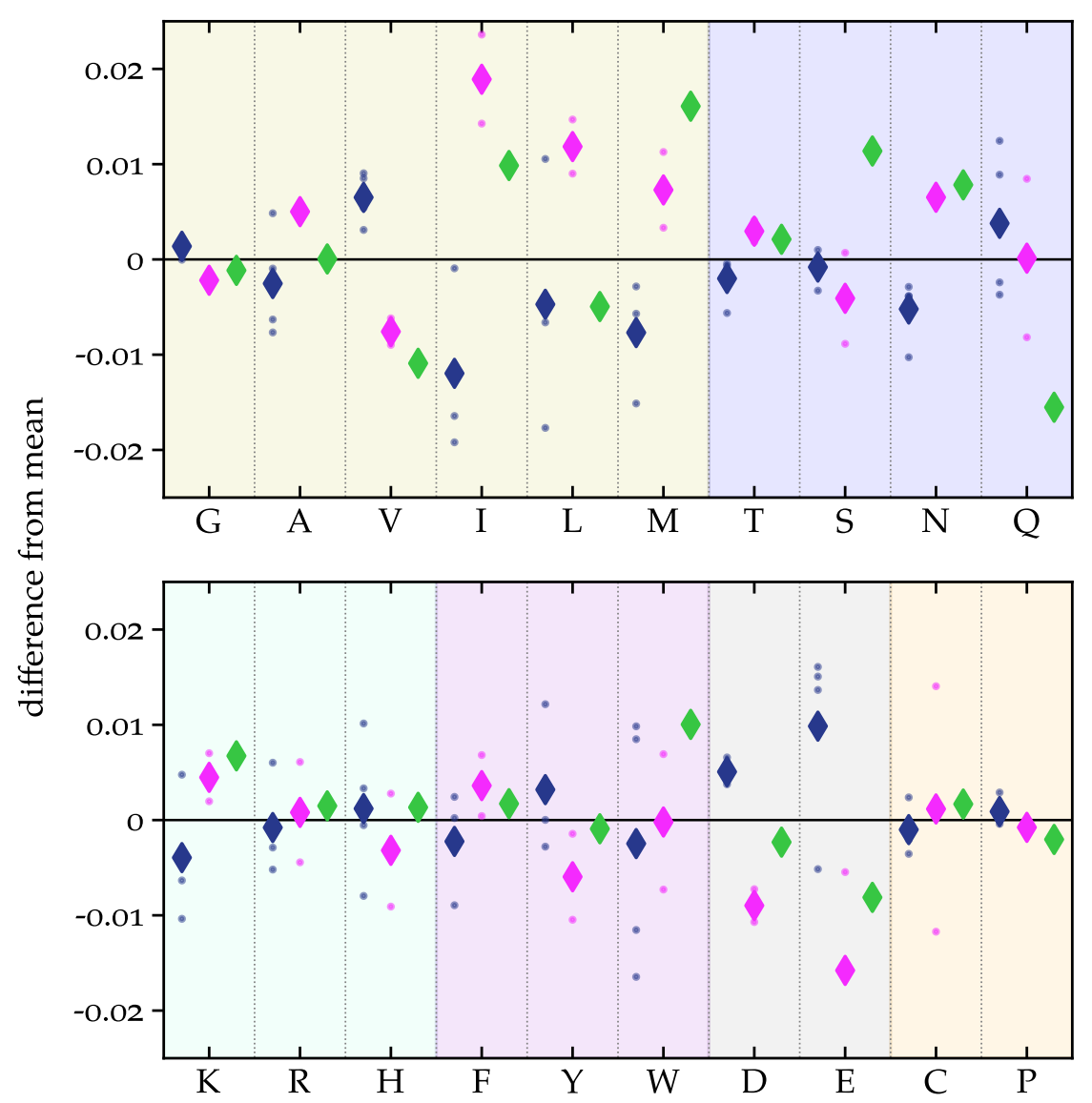

Figure 3.24: Differences in $\alpha$-helix amino acid content of Octadecabacter groups. The average number of each amino acid in core-protein positions predicted to be part of a helix was related to its average number outside predicted helices for each group. The plot shows the difference between these mean ratios and the base (mean) ratio over all seven Octadecabacter strains with similar GC-content (Table 3.1). Octadecabacter groups and colours as defined in Section 3.2 (I, II, and IV); amino acid groups are the same as in Figure 3.23. 
A total of 591 metagenomes on the mg-RAST platform met the inclusion criterion for the analysis (Section 2.12.1), of which 559 could be successfully downloaded and mapped. The metagenomes contained either reads, or assembled contigs, with a total of $1,342,811,866$ sequences assigned to the bacterial kingdom by mg-RAST. Mapping returned 359,928 sequences which aligned to the Octadecabacter genomes. The filtering procedure focusing on distinction between Octadecabacter groups (Section 2.12.3) excluded 20,388 hits $(\approx 5.6 \%$ ), whereas 23,983 sequences $(\approx 6.7 \%)$ were excluded when pairwise comparisons between all Octadecabacters were considered. All relevant data are also provided in the supplementary files (see Appendix A.1).

For 522 metagenomes, geographic coordinates were available. They stemmed from 214 distinct sampling points, at 113 of which several dates, size fractions, depths, etc. were sampled, leading to 308 metagenomes with duplicated coordinates. Eighty-two of the stations contained metagenomes with sequences mapping to at least one Octadecabacter, while 132 stations returned no hits (Figure 3.25).

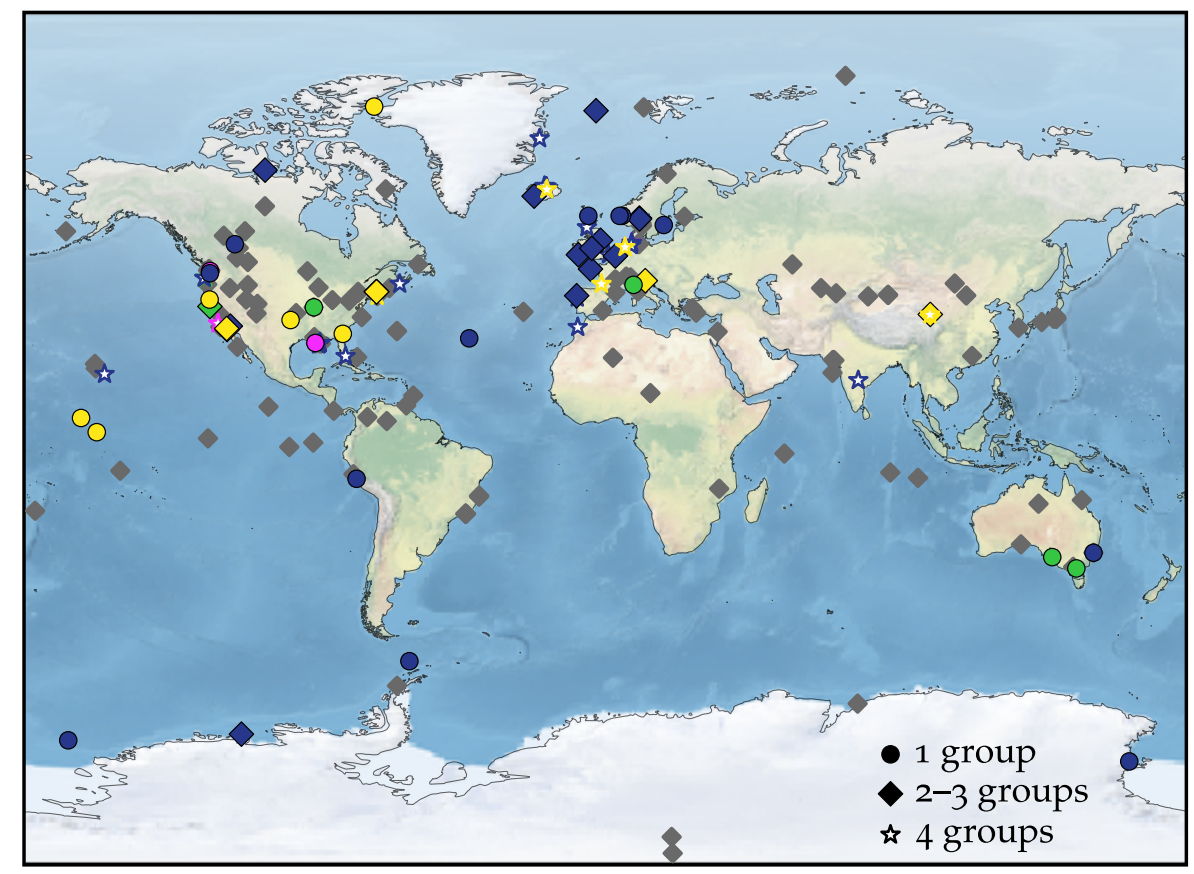

Figure 3.25: Octadecabacter groups at metagenome sampling sites. Where multiple metagenomes were present for a single sampling point, they were merged using the highest relative abundance of each group in any of the samples. Shown are sampling sites with reads mapping to no Octadecabacter (132 stations, grey diamonds), one specific Octadecabacter group (24 stations total, circles of correspondent colour), 2-3 Octadecabacter groups (diamonds, coloured according to most abundant group) or to all Octadecabacter groups (37 stations, stars coloured according to most abundant group). 
Polar Octadecabacters (group I) were detected in 117 metagenomes at 69 sampling sites and are the most widely distributed of the four groups (Figure 3.25). Likewise, they are often the most abundant of all detected Octadecabacter groups (Figure 3.26). Groups II, III, and IV were detected in 74, 86, and 88 metagenomes at 51,49, and 60 stations, respectively. At 37 sampling sites, metagenome sequences mapped to all four Octadecabacter groups, while 24 stations featured one group exclusively (Figure 3.25).

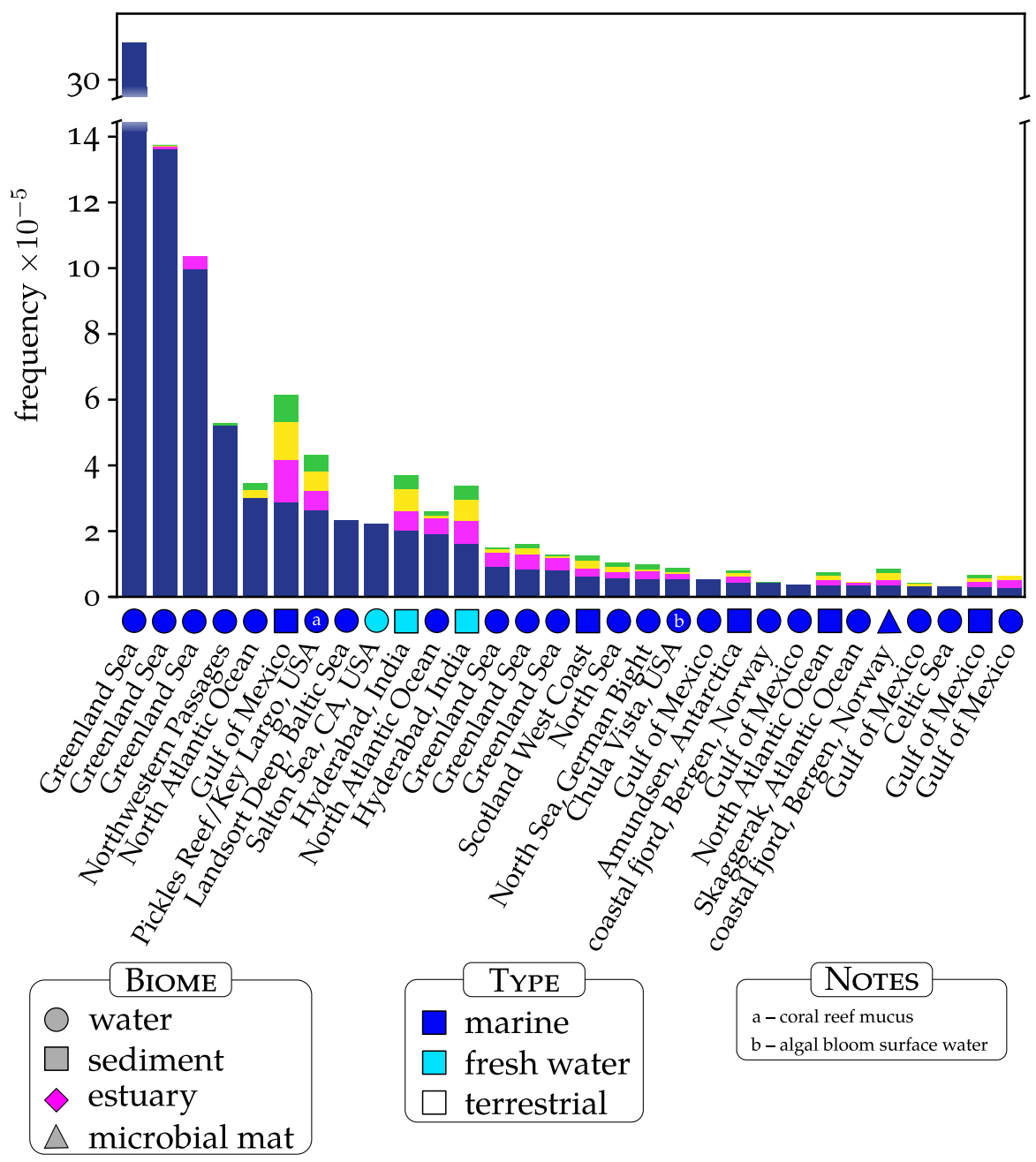

(a) Octadecabacter group I

Figure 3.26: Metagenomes ranked by abundance of Octadecabacter groups. The number of nucleotides in reads mapping to a specific Octadecabacter group, divided by the total number of nucleotides assigned to the bacterial kingdom, is plotted as the frequency. Sub-figures (a) to (d) list the 30 metagenomes, in which groups I to IV occur with the highest frequency, respectively, along with information on the biome, sample type, and sampling location.

The vast majority of metagenomes with a high frequency of nucleotides mapped to groups I and II originates from marine water samples, with marine sediment being the second most frequent biome 

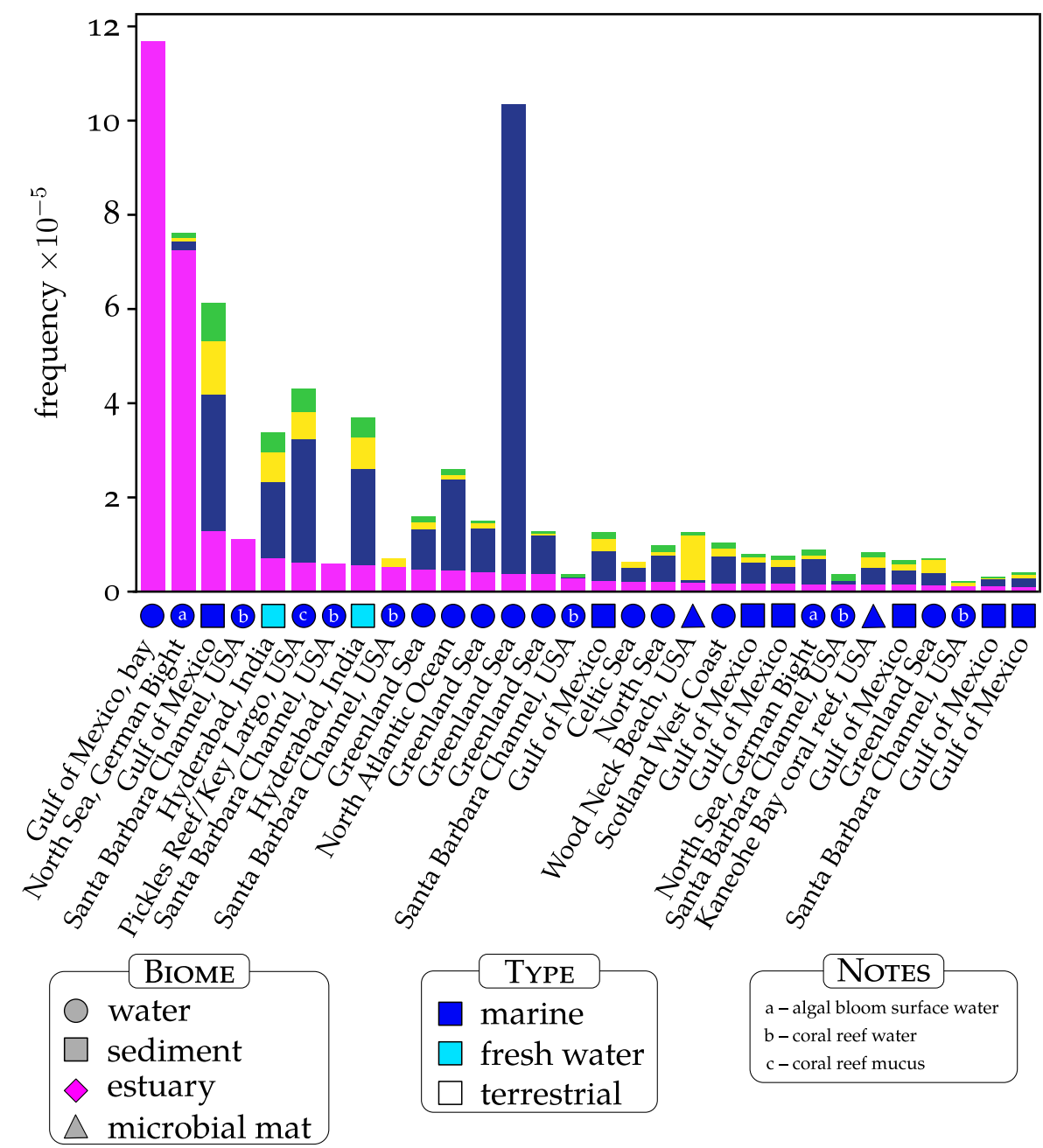

(b) Octadecabacter group II

Figure 3.26: Metagenomes ranked by abundance of Octadecabacter groups (continued)

(Figures 3.26a and 3.26b). Conversely, habitat types are more evenly distributed among the metagenomes with nucleotides mapping to groups III and IV, showing a higher number of microbial mats, estuaries, or terrestrial samples (Figures 3.26c and 3.26d). These groups' frequencies are, however, generally much lower. Groups I and II, on the other hand, predominantly feature in 4 and 2 of the 6 metagenomes with the highest frequency of nucleotides mapped to Octadecabacters, respectively.

While most metagenomes listed in Figure 3.26 contain reads mapped to multiple, or all Octadecabacter groups, those which show the highest frequency of an individual group are usually exclusive to that group. Therefore, despite the frequent co-occurrences, it is possible to identify some habitat preferences of the different groups. For example, group I most prominently features in water samples of the Greenland Sea and the North Atlantic Ocean in general, i. e. water samples of high 


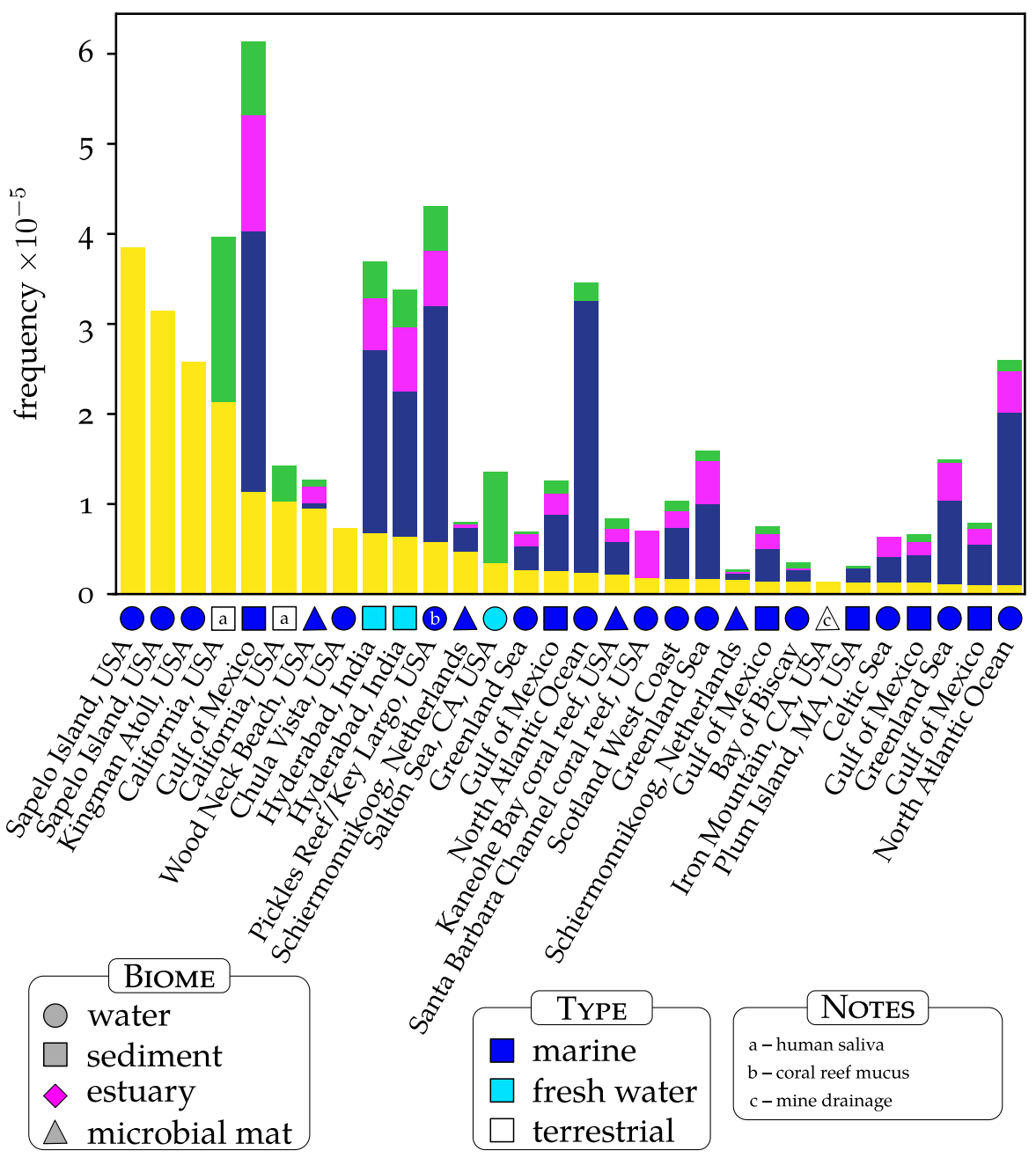

(c) Octadecabacter group III

Figure 3.26: Metagenomes ranked by abundance of Octadecabacter groups (continued)

latitudes, where other Octadecabacter groups seem to be nearly absent (Figure 3.26a).

Group II has more hits in metagenomes from coastal areas and bays, and notably features in water samples of coral reefs or algal blooms, where it likewise is often the only detected group (Figure 3.26b). These regions are mostly positioned at intermediate latitudes, but the group also seems to occur to some extent at higher latitudes, e.g. in water samples of the North Atlantic Ocean.

Group III shows the highest frequencies of mapped nucleotides in several water samples from low latitudes (Figures 3.25 and 3.26c). Nonetheless, there are also highly similar sequences present in metagenomes from higher latitudes. Interestingly, it is the only group detected in one of the northern-most samples included in this analysis, a freshwater lake microbial mat on Ellesmere Island, Canada (Figure 3.25). 

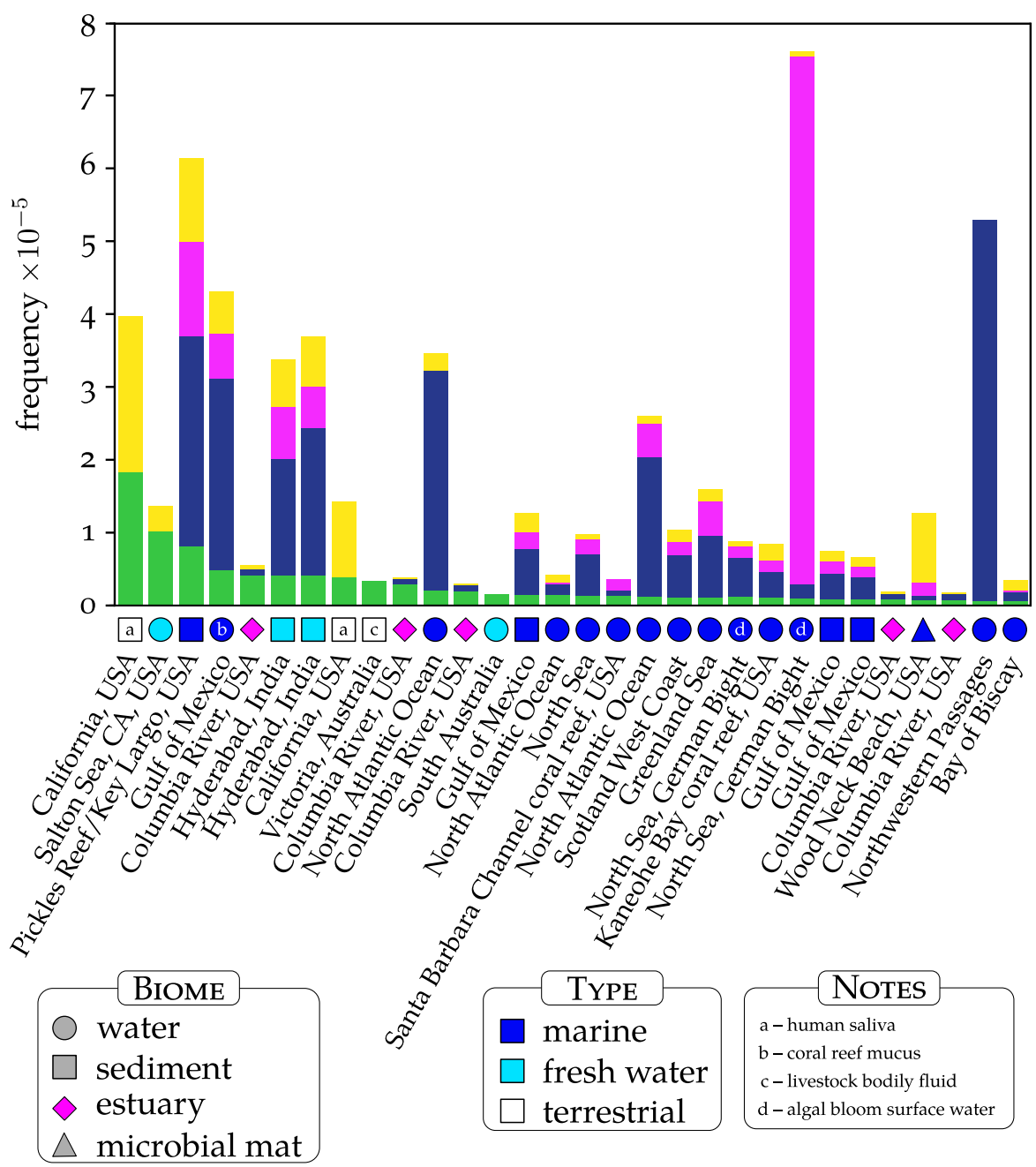

(d) Octadecabacter group IV

Figure 3.26: Metagenomes ranked by abundance of Octadecabacter groups (continued)

Group IV generally exhibits the lowest number of mapped reads within the Octadecabacters, and among the metagenomes with the highest nucleotide frequency of this group, there seems to exist no particular habitat preference (Figure 3.26d). Notably, it is featured more prominently in some of the tested estuarine metagenomes than the other groups.

In general, when evaluating how many of the candidate metagenomes actually contained reads mapping to Octadecabacters, some differences between the habitat types are noticeable. The sea water and "marine habitat" biome categories, for the latter of which no further details were provided in the metadata, contained among the most metagenomes, based on the biome and sample type descriptions. However, only 25 to $50 \%$ of these gave hits in the stricter mapping procedure (Figure 3.27). On the other hand, all samples of marine and freshwater 


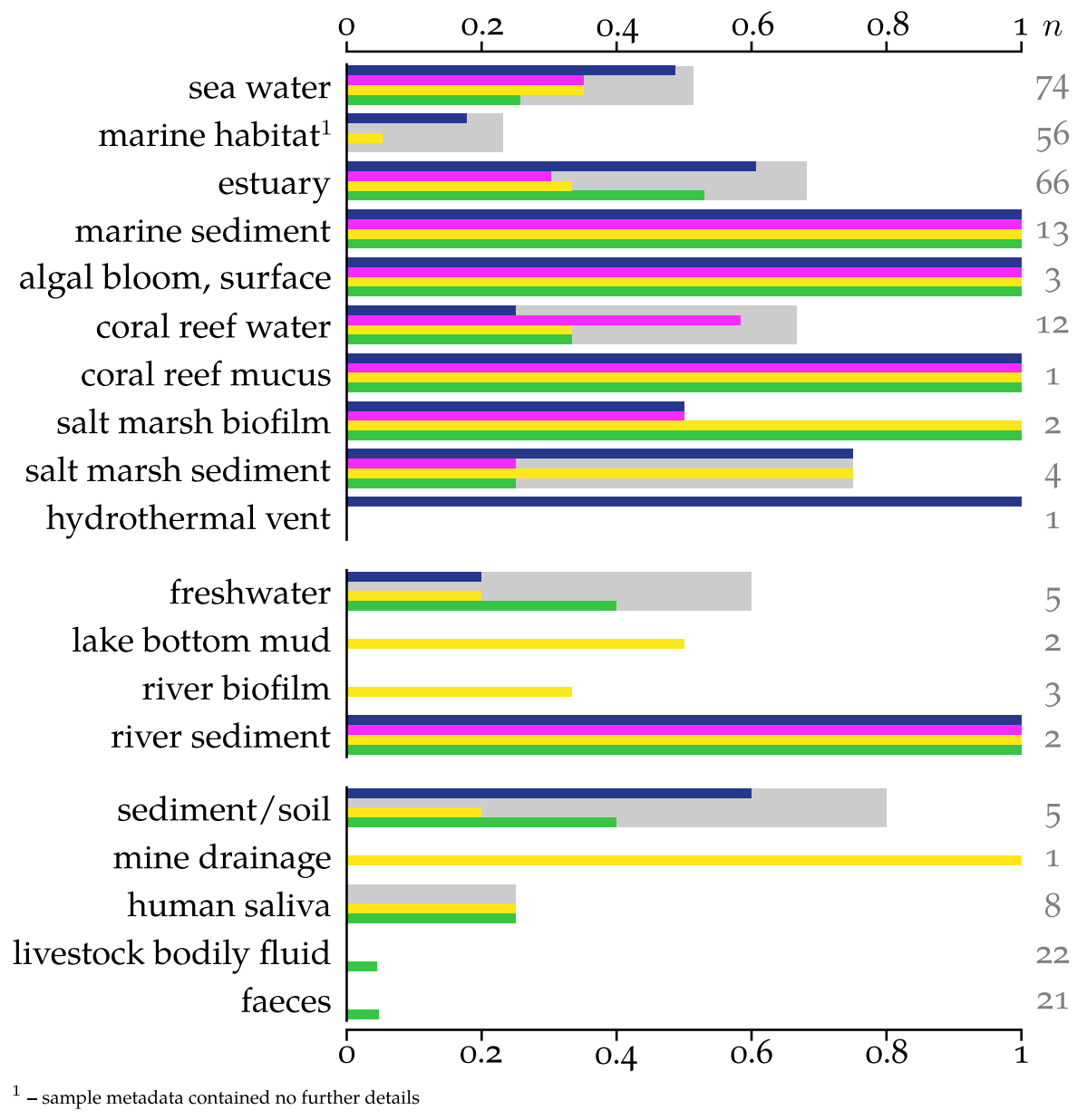

Figure 3.27: Octadecabacter group occurrence in different biomes. For each biome/sample type represented by a metagenome in this analysis, the proportion of metagenomes in which individual Octadecabacter groups were detected is plotted (colours as in Section 3.2, groups I, II, III and IV). Grey numbers $(n)$ give the total amount of metagenomes for each biome/sample type. Where more than one group occurs in a habitat, a grey bar indicates the fraction of metagenomes with reads mapping to any group. Biomes are sorted into marine (top), freshwater (middle), and terrestrial (bottom) habitats.

sediment, in which mg-RAST assigned sequences to the Octadecabacter genus, also returned mapped reads.

Agreement in predictions of Octadecabacter occurrence between mg-RAST and the mapping approach used here is generally high for marine biomes ( 70 to $100 \%$, with the exception of sea water and "marine habitat"). It is, however, lower in freshwater and terrestrial biomes, where mg-RAST predicted Octadecabacter-related sequences in 12 and 57 metagenomes, respectively (including bodily fluids and faeces, Figure 3.27). Read mapping returned hits in 7 and 9 of these metagenomes, respectively. In over half of the sample types, reads mapped only to either group III, or group IV. Sediment (both freshwater and terrestrial), and fresh water samples pose a notable exception 
in this regard, having a higher proportion of metagenomes with reads mapping to multiple Octadecabacter groups (Figure 3.27). 
Chapter 3 presented several genomic characteristics of Octadecabacter clade bacteria and made initial comparisons to other members of the Roseobacter group. These data enable an investigation, in how far such characteristics generally relate to differences in lifestyle between the analysed strains, and whether Octadecabacters constitute special cases among the roseobacters.

Having reconstructed the group's most likely phylogeny using a phylogenomics approach is particularly useful in this regard, as it allows comparison of similarities in gene content to phylogenetic relation in potentially large groups of genomes. Section 4.1.1 will evaluate the obtained phylogenies reliability and discuss their congruence with observed lifestyles and genetic divergence. The discussion will cover both the Roseobacter group as a whole, and the Octadecabacters in particular.

Variability in gene content is generally well captured by analysing a group's pan-genome. Such an analysis provides standardised characteristics, which can be readily compared between different organism groups. In Section 4.1.2, results of the Octadecabacters' pan-genome analysis will be put in relation to other clades of the Roseobacter group.

Finally, genus delineation is a widely debated topic, and can be particularly problematic for the functionally diverse roseobacters (see Sections 1.4 and 1.5). Section 4.1.3 will address this problem, using the example of the Octadecabacter clade to evaluate and compare several metrics proposed for use in genus delineation.

\subsubsection{Phylogeny}

As stated in Section 3.2, a super-matrix-based MLSA of the Roseobacter group core-genome generally reproduced the topology observed in other studies [59, 60]. The Octadecabacters form a distinct and highly supported clade (compare Figure 3.2, p. 30), with isolate NH9-P7 being the earliest branching and therefore most distant member, followed by $P$. jejudonensis. The polar isolates cluster together with high bootstrap support, and also form a common sub-clade with the temperate members O. temperatus and O. ascidiaceicola. However, phylogenies derived from super-matrices are under criticism for not necessarily reflecting the most prevalent topology, as well as rendering bootstrap supports misleadingly high [286]. 


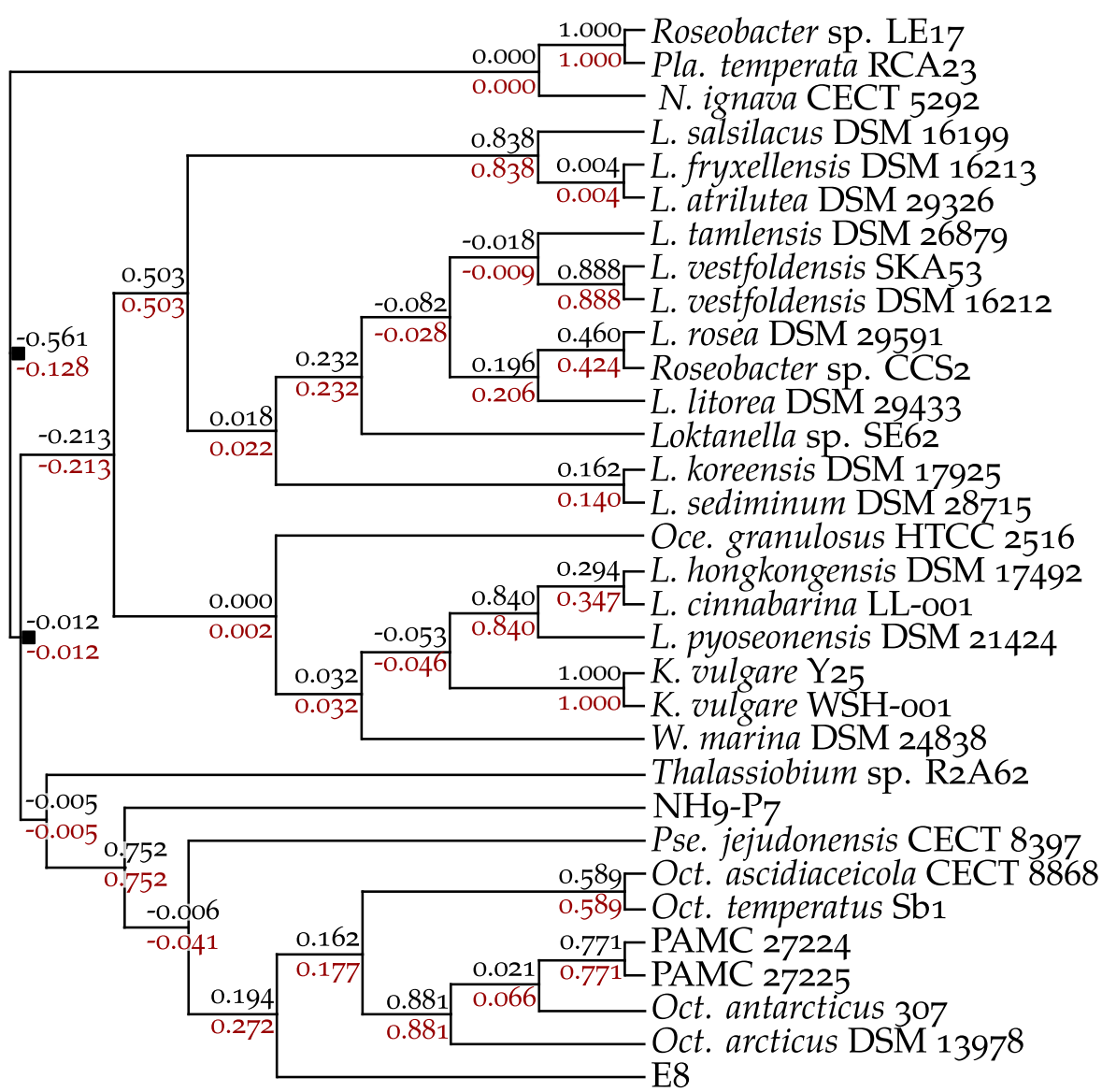

Figure 4.1: Reassessment of bipartition support within the super-matrixbased tree topology, using IC and TC values. The tree topology was taken from Figure 3.2, and support values at each bipartition were re-estimated as IC (black) and TC (red) by comparing the MLSA with 139 single core-gene phylogenies (see Section 2.6 for details).

A more realistic estimate of bipartition support can be derived from the frequency in which the bipartitions occur in the ML phylogenies of the individual genes. More precisely, the frequency of each bipartition can be related to the two most frequent conflicting bipartitions, which results in the information theory-based internode certainty (IC) and tree certainty (TC) values [174]. Values close to o indicate that the next two most frequent topologies are almost as prevalent as the one shown in the tree, whereas values close to 1 show the absence of such conflicts (negative values mean that at the respective site, other bipartitions occur more frequently altogether).

When comparing the 139 core-gene phylogenies with the supermatrix-based one in this manner, it is obvious that the high MLSA bootstrap support values are exaggerated, implying a false sense of certainty in the final tree topology (Figure 4.1). While the formation of an Octadecabacter clade, distinct from their closest relative, Thalassiobium, is still well supported (IC value of 0.75 ), the branching order between the more distant members $P$. jejudonensis, isolate $\mathrm{NH}_{9}-\mathrm{P}_{7}$, 
and isolate E8, appears to be much less reliable than indicated by the bootstrap analysis (signified by low IC values of 0.006 to 0.2 at the respective bipartitions). Common ancestry of the four polar isolates, O. temperatus, and O. ascidiaceicola is seemingly conflicted in the individual Roseobacter group core-gene trees (low IC of 0.16), which agrees with the network representation of 1,513 Octadecabacter clade coregene phylogenies (Figure 3.3). At the same time, both the existence of a polar clade, and the grouping of O. temperatus and O. ascidiaceicola, maintain a high support.

Over the rest of the Roseobacter group topology, certainty is comparatively low: multiple negative IC values indicate that the MLSA phylogeny does in fact not represent the phylogeny of most of the component genes (see Figure 4.1).

It should be noted that the genes used for MLSA were selected solely based on their classification as single-copy core-genes, and not individually tested for the strength of their respective phylogenetic signal. Consequently, part of the observed uncertainty may simply result from phylogenetic noise within the individual gene trees. In addition, a super-matrix-based phylogeny always constitutes a compromise between conflicting phylogenetic signals of its component genes, and is therefore inherently uncertain by necessity. This becomes clear when analysing the Robinson-Foulds (RF) distances between the supermatrix-based MLSA phylogeny and the individual gene trees, and comparing them to the pairwise RF distances of the individual trees among each-other. In this case, the median normalised RF distance to the MLSA phylogeny is lower (by 0.13) than the median normalised distance of pairwise comparisons of the individual gene trees (see Figure 4.2). Thus, the MLSA phylogeny appears to present an adequate compromise between the conflicting single gene trees, minimising the overall distance, while not necessarily agreeing with the majority of their topologies. For this reason, it is appropriate to use this phylogeny as the basis of ancestral state reconstruction and the phylogenetic birth-and-death model, as described in Section 2.9, although the huge uncertainties concerning the bipartitions (especially deeper ones) should be kept in mind when discussing findings which rely on phylogenetic data.

Roseobacter group members are widely distributed, mostly in marine environments, where they occupy a variety of ecological niches [62]. Even within the genera of this group, different species are often found to dwell in different micro-environments, which may be as diverse as planktonic, biofilm, or host-associated habitats (compare Table A.1, p. 125). In fact, some argue that this colonisation of new micro-environments, and the subsequent decrease of gene transfer between populations, could constitute a mechanism of speciation [287]. The high genetic flexibility frequently observed in Roseobacter group members likely makes such a transition easier, and thus contributes to this group's adaptability [69]. 


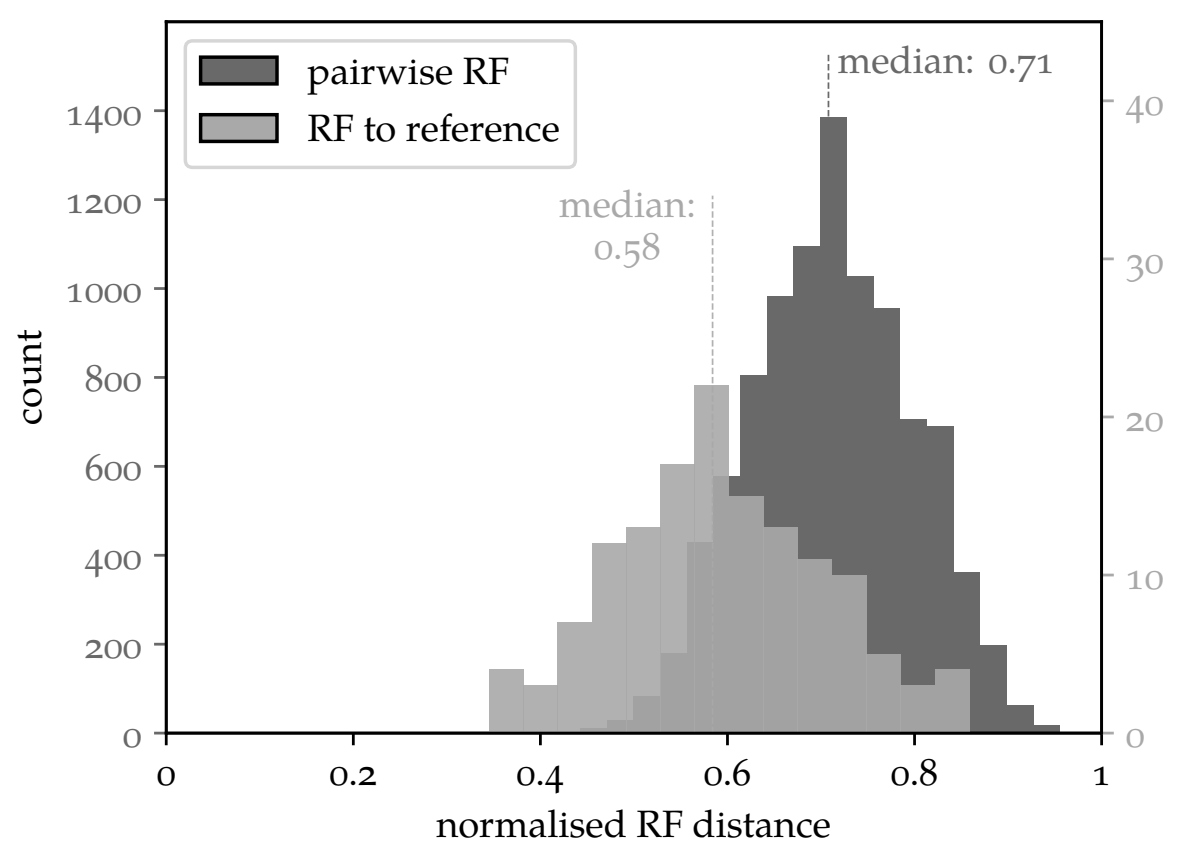

Figure 4.2: RF distances between individual core-gene and super-matrix-based phylogenies in the Roseobacter group. Pairwise RF distances were calculated for all 139 individual core-gene phylogenies, and their distribution is shown in dark grey (left axis). The distribution of their distances to the super-matrixderived phylogeny is depicted in light grey (right axis).

Despite such varying lifestyles, gene content-based hierarchical clustering showed high consistency with the MLSA phylogeny (see Figure 4.3). Clades defined in ref. [59] are largely conserved between both trees, with only few individual organisms grouping differently in the gene content-based clustering. Most differences in bifurcations between the two trees are restricted to deep nodes, at which point the aforementioned uncertainty in the MLSA branching forbids exact comparisons. Consequently, vertical inheritance appears to dominate gene content over horizontal acquisition, i. e. closely related Roseobacter group members possess a distinct genetic coherence. Notably, this coherence does not seem to be limited to the core-genome. The removal of core-genes within clades throughout the Roseobacter group and subsequent hierarchical clustering results in a tree, which is even more congruent with the MLSA phylogeny (Figure A.3, p. 133; normalised RF distance of 0.47 without sub-clade core-genes, versus 0.59 when including core-genes). This is even the case in groups like the polar Octadecabacters, which are known to have highly flexible genomes with strong potential for lateral gene transfer (LGT).

The cloud-, and shell-genomes reflect an organism's adaptations to its particular micro-habitat [288]. As these adaptations occur predominately via acquisition of laterally transferred genes [289], these acquired genes will primarily make up the shell-genome. Since most roseobacters possess genes encoding for GTAs (Section 1.3), within- 
lineage transfer of genetic material may have a role in shaping individual strains' gene contents [6o]. Nonetheless, similarities in cloud- and shell-genome content do not reflect similar habitats, or geographic proximity ${ }^{1}$ of the compared Roseobacter group strains (Table A.1), but, as stated above, rather follow the phylogeny (Figure A.3). Despite the higher number of genomes compared here than were available to Newton et al. [6o], reliably testing the idea of a common roseobacter gene pool, the exchange of which facilitates environmental adaptation, would likely still require more sequenced representatives [6o].

In the phylogenetic analysis based on the Octadecabacter core-genes, a network representation was chosen to directly visualise conflicts in phylogenetic signal between the individual gene trees (Figure 3.3). With regard to the network's overall fidelity, an analysis of RF distances, equivalent to the one performed for the Roseobacter group MLSA, showed a lower median distance of the individual gene phylogenies to the network than to each-other (0.33 vs. 0.50, respectively), indicating that the network may be an accurate representation of the phylogenetic relationships within the Octadecabacters.

Clustering the nine strains based on their gene content, and thereby their potential functional adaptations, revealed some notable differences (Figure 3.3). While the polar clade and the clade formed by $O$. temperatus and O. ascidiaceicola remain individually unchanged by gene content clustering, they no longer form a common larger cluster as in the core-genome phylogeny (albeit at low IC support). Within the polar species, $O$. antarcticus clusters with $O$. arcticus, rather than with the other Antarctic isolates. This may reflect their similar lifestyle: O. arcticus and O. antarcticus occur in sea water as well as in sea ice, while the PAMC isolates were procured from marine sediments (see Section 1.4). Notably, this particular bifurcation is also conflicted in the network, meaning that a significant proportion of single gene phylogenies puts $O$. antarcticus in closer relation to $O$. arcticus than to the PAMC isolates.

Vollmers et al. attributed the high observed similarity between both strains to a mutual genetic exchange, postulating a potential connection between both Arctic and Antarctic populations [77]. Apart from the coherent grouping of the polar Octadecabacter clade members, lifestyle does not seem to be a good predictor of content-based similarity, neither does geographic proximity: O. temperatus and O. ascidiaceicola are stably grouped together, despite the fact that they were isolated from distant parts of the world, and from different marine micro-environments (free living fraction of the water column for the former, sea squirt-associated for the latter, see Section 1.4). Likewise, both the isolates from Korea (O. ascidiaceicola and P. jejudonensis) and

1 hierarchical clustering based on the geographic distance of the available isolation coordinates (Figure A.4) returned a tree with an RF distance of 0.96 to the MLSA, as well as both the gene content and core-genome-reduced gene content trees 


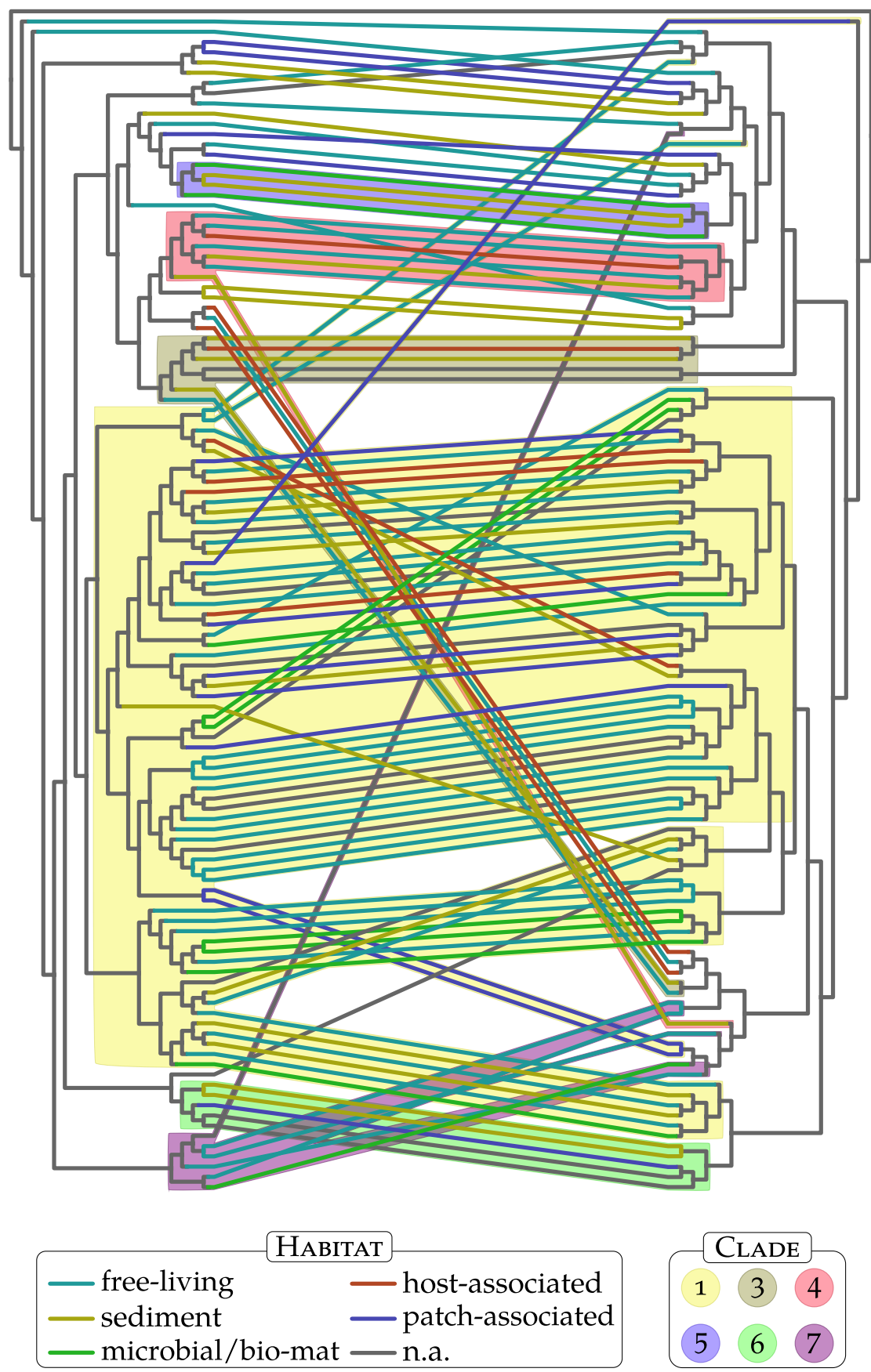

Figure 4.3: MLSA phylogeny of 115 roseobacter genomes plus outgroup on the left, as given in Figure A.2, versus hierarchical clustering of the same genomes on the right, based on pairwise Jaccard distances of OG presence and absence. Clades defined in ref. [59] are coloured as in Figure A.2. Each strain's lifestyle or preferred type of habitat was estimated from the source publication (see Table A.1), and branches are coloured accordingly. RF distance between both trees is 0.59 , determined using ete 3 [177]. 
those from the North Sea (O. temperatus and isolates $\mathrm{NH}_{9}-\mathrm{P}_{7}$ and E8) do not form common clusters.

This reflects the above observations for the whole Roseobacter group, namely that phylogeny is a better predictor of content-based similarity than similar lifestyles. In the present case, isolate $\mathrm{NH}_{9}-\mathrm{P}_{7}$ and P. jejudonensis are both phylogenetically distant (Figure 3.2), as well as highly dissimilar from the other clade members on the sequence level (Figure 3.1). Due to this distance, vertical inheritance of genes may determine their position in content-based clustering, rather than similarities in lifestyle. As with the whole Roseobacter group, a higher number of available genome sequences would allow for more conclusive statements, how far geographic proximity and/or similarity in lifestyle correlate with phylogenetic relation. It should also be noted that the circumstances under which an organism is isolated do not necessarily reflect its preferred lifestyle, and this information is therefore associated with some uncertainty.

In general, the observed distances in gene content between the Octadecabacters are relatively high, which may be partly owed to the fact that singletons were included in the distance calculation. The polar Octadecabacters are, as a group, most divergent in this regard. Apart from them, strain E8 is notably the single most distantly branching isolate of the Octadecabacter clade (based on its distance to the tree root). This is also reflected in both groups' singleton fractions (Figure 3.6), as will be discussed in the following section.

\subsubsection{Pan-genome analysis}

The genomic diversity of a group of organisms can be assessed by analysing the correlation between the total number of different orthologous groups (OGs) (the so-called pan-genome) and the number of compared genomes [290]. This correlation usually follows a power law. Pan-genome analysis of the Octadecabacters revealed that the corresponding fitted power law curve does not reach saturation (exponent $0<\mathrm{b}<1$, see Table 3.3, Section 3.3.1), a property which defines an open pan-genome [290]. This means that the currently available Octadecabacter clade genome sequences do not yet fully represent the clade's inherent genetic diversity [188]. Instead, when added to the analyses consecutively, each new genome contributes $\approx 700$ novel genes to the clade's pan-genome (Figure 4.4), reflecting unique adaptations to their different habitats (compare Section 1.4), and indicating high flexibility of the accessory genome for acclimatisation to specific niches [291]. This adaptability is, however, not more pronounced in the Octadecabacters than in other clades within the Roseobacter group. The analysed comparison clades show similar power law exponents (see Table 3.3), and the number of novel OGs added to the pan-genome by the successive addition of each new species does not differ significantly 


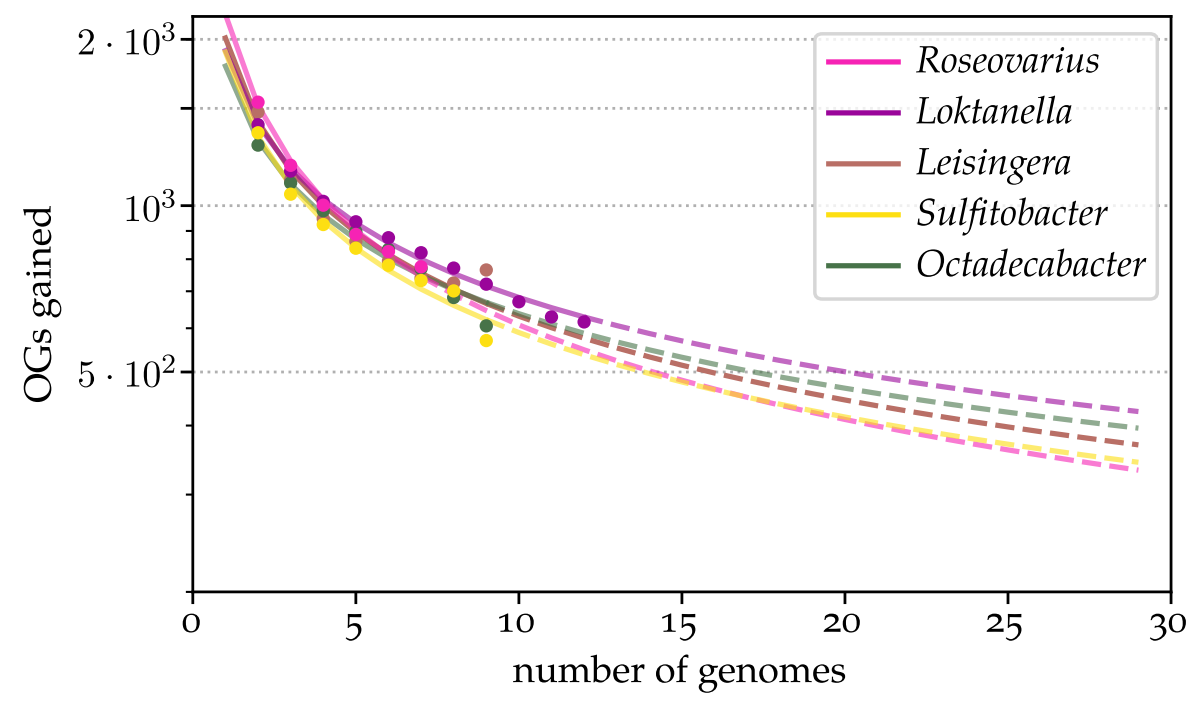

Figure 4.4: Change in pan-genome size in relation to the number of comparison genomes for Octadecabacters and other comparison clades of the Roseobacter group. Clades were selected based on similar phylogenetic branching depth (see Table 3.2). Median values fitted to a power law as in Equation (2.1), p. 23. Dashed lines are extrapolations based on the parameter estimates.

between any of the investigated clades (compare extrapolated curves in Figure 4.4). Consequently, they possess equally broad pan-genomes, which supports the frequently stated assumption that high genome plasticity and genetic adaptability are general traits of the Roseobacter group [69, 93] (Section 1.3).

The Roseobacter group possesses an open pan-genome, which comprises >70,000 OGs in 114 genomes (Figure 3.5 and Table 3.3). Generally, open pan-genomes are common for bacteria at the genus [292-294], and the species level [188]. The increase in number of genes by successively added genomes is a relatively simple metric that can easily be compared between different studies. Interestingly, this metric is notably higher in the comparison clades of the Roseobacter group, including the Octadecabacters, than for other analysed bacterial groups [188, 292294]. One should keep in mind that observable pan-genome trends are dependent on the number and nature of the compared genomes [188]. Due to the limited amount of data for the roseobacters, and Octadecabacters in particular, the observed curves can only be taken as a trend. While the estimated curve parameters seem robust due to their low variances (Table 3.3), they do not reflect the inherent uncertainty introduced by the selection of comparison genomes. In order to more conclusively demonstrate that the Octadecabacters possess a higher genomic diversity than other comparison groups, the analyses would therefore need to be repeated with more data. In addition, an open pan-genome on the genus level can mask the closed pan-genomes of one or multiple constituent species [293]. Given more genomic data, it would be interesting to see whether lineages exist within the roseobac- 


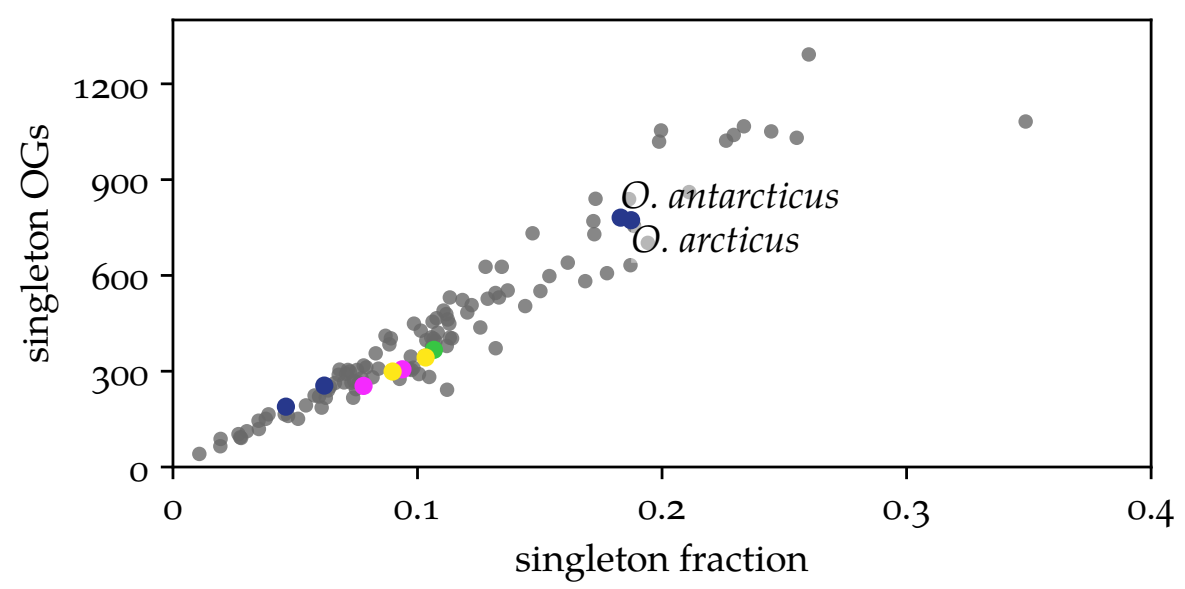

Figure 4.5: Occurrence of singletons in Roseobacter group genomes. The number of singletons (i. e. orthologous groups (OGs) exclusive to one genome) is plotted against their fraction of total OGs for each comparison genome listed in Table 2.2, excluding the outgroup genome Parvularcula bermudensis and Phaeobacter sp. LSS9. Octadecabacters are highlighted in colours corresponding to their groups defined in Section 3.2.

ters, which do not fit into the marine generalist scheme, and if so, at which level of phylogenetic divergence this behaviour would emerge.

Apart from allowing an estimation of general genome diversity, gene orthology information enables a detailed look at the specific distribution of genes within a selected group of organisms. To that end, the distribution of genes in the core, cloud, and shell-genomes of the Octadecabacters, as well as several comparison clades, was analysed (Section 3.3.1). As noted there, O. arcticus possesses the largest shellgenome fraction among the Octadecabacters (Figure 3.6). An earlier study found both $O$. arcticus and $O$. antarcticus to contain the highest number of unique genes among 32 Roseobacter group genomes [60]. Among the 115 comparison genomes used here, they do not represent extreme cases in this regard, as multiple other strains possess more singletons, as well as a higher fraction of unique OGs in their genome (Figure 4.5).

Nonetheless, the core-genome still represents the largest genome fraction in all Octadecabacters $(40 \%$ in the polar Octadecabacters and $50 \%$ in the rest, Figure 4.6). The shell-genome of O. arcticus makes up $\approx 30 \%$ of its CDSs. Other clades within the Roseobacter group show similar variation in the three categories (Figure 4.6). For most of the respective genomes, the core-genome comprises 30 to $50 \%$ of the total number of CDSs (varying according to genome size). Although the Octadecabacters tend towards relatively smaller cloud-, and larger shellgenomes than their relatives, they do not constitute extremes in any of the two categories. For example, some Roseovarius clade members contain even smaller cloud-genomes than the Octadecabacters, and the shell-genome fraction of one Loktanella clade strain exceeds that of $O$.

singleton - gene exclusive to one genome of a comparison group 


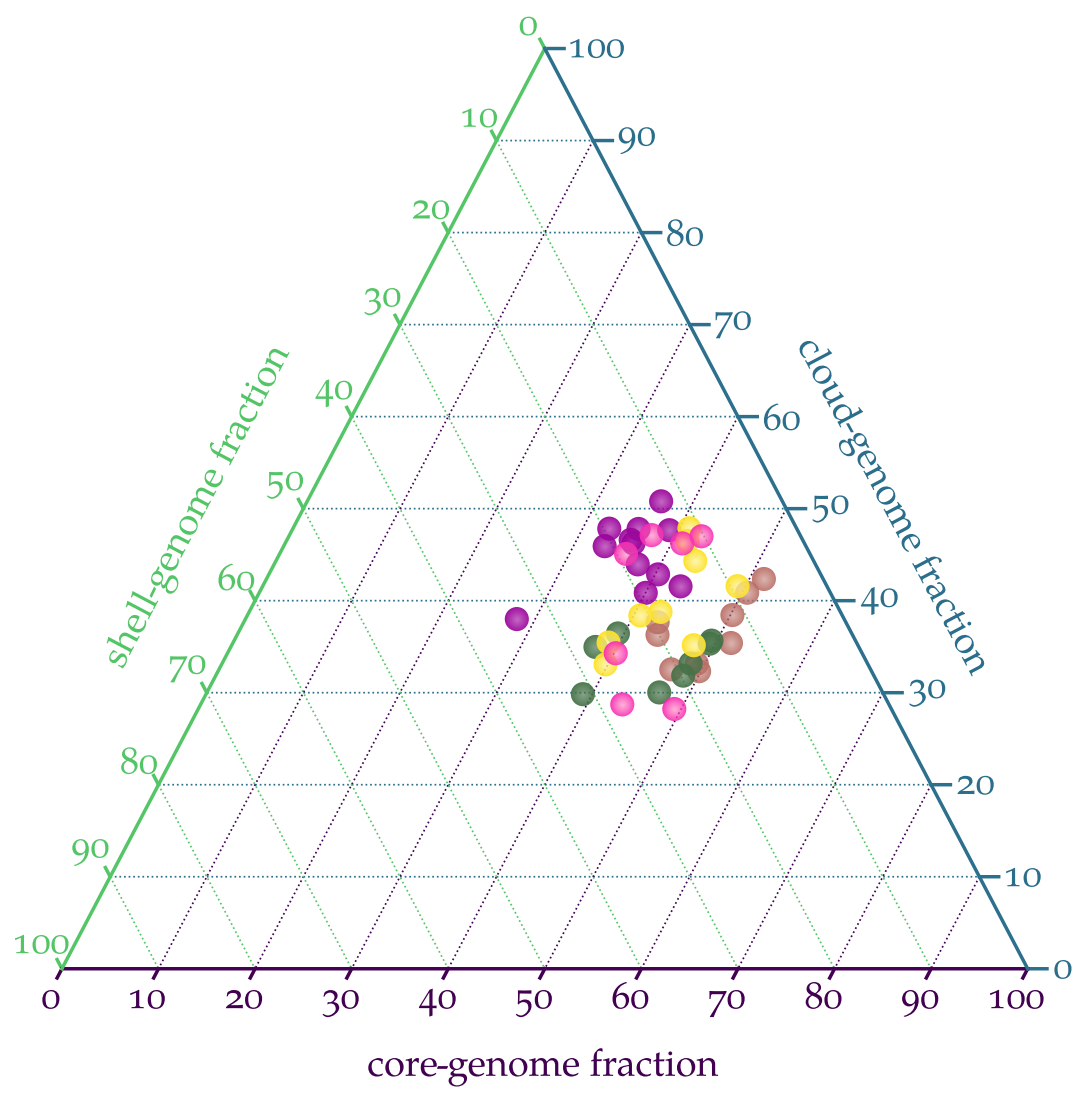

$$
\begin{array}{ll}
\text { - Loktanella Sulfitobacter • Octadecabacter } \\
\text { - Leisingera } \odot \text { Roseovarius }
\end{array}
$$

Figure 4.6: Core-, cloud-, and shell-genome fractions (in percent) among selected Roseobacter group lineages. Comparison lineages were selected based on similar branching depths compared to the Octadecabacter clade (Table 3.2). In the case of extremely similar genomes (i. e. near-zero branch lengths in the reference phylogeny), such as the two PAMC isolates, only one respective representative was included.

arcticus. The exact genome category distribution is markedly different between the Octadecabacters and the closely related neighbouring Loktanella clade: Loktanella strains tend towards larger cloud-genomes, at approximately equal expense of both the shell-, and core-genomes.

\subsubsection{Genus delineation within the Octadecabacter clade}

The extensive functional divergence discussed in the previous sections complicates a sensible delineation of genera within the Octadecabacter clade and Roseobacter group in general. The re-classification of $O$. jejudonensis as Pseudooctadecabacter was, for example, mainly derived from its phenotypic differences to three other clade members in the polyphasic analyses [79]. Based on the genomic flexibility and range of habitats, however, such phenotypic differences can accumulate quickly, and 


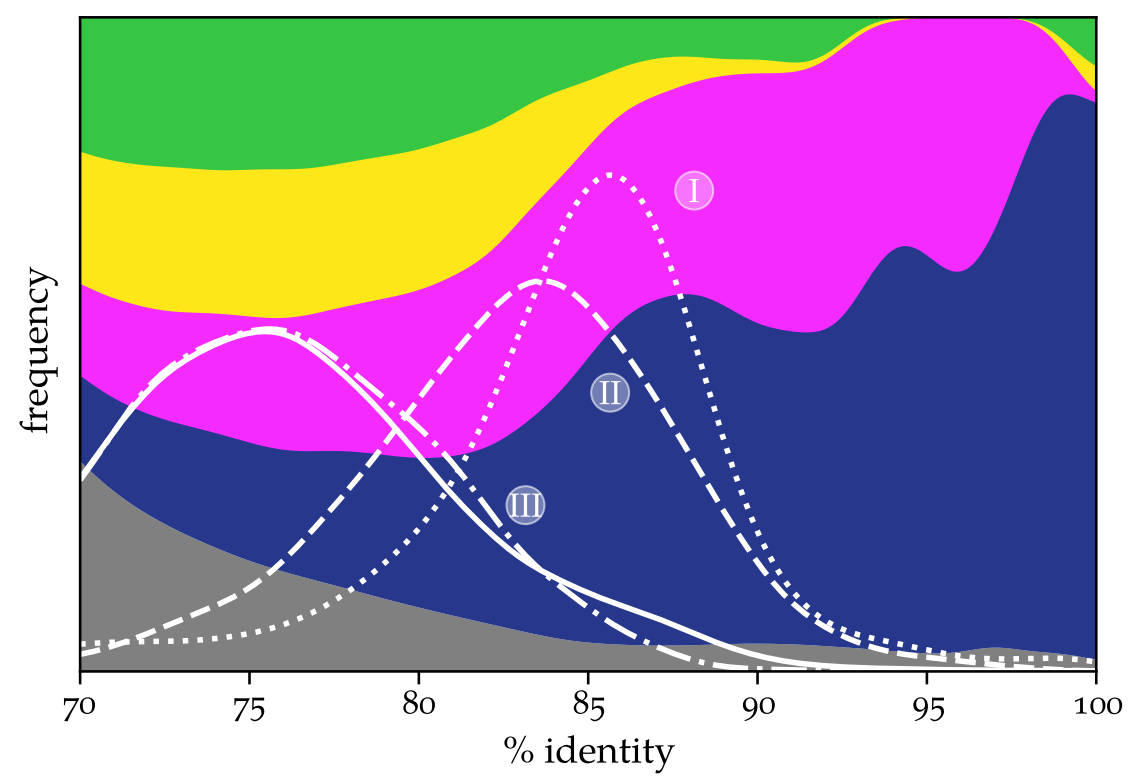

Figure 4.7: Nucleotide identity distribution within Octadecabacter groups. White curves show distribution of nucleotide numbers over \%-identity values for pairwise alignments of 1 ,ooo nt-fragments within Octadecabacter groups I to III (labelled accordingly), and between all nine genomes (solid line). Background visualises how Octadecabacters compare to other roseobacters (Table 2.2), showing what proportion of nucleotides at a particular \%-identity value to any Octadecabacter stem from a roseobacter (grey), or another Octadecabacter (coloured according to its group). Note that rRNA genes were excluded from the comparison (Section 2.12).

traits such as carbon compound utilisation, among others, show a high phylogenetic dispersion [295]. Digital DNA-DNA hybridisation (DDH) demonstrated a low similarity between $P$. jejudonensis, isolates $\mathrm{NH}_{9}-\mathrm{P}_{7}$ and E8, and the other clade members (Figure 3.1), and on this basis it seems justifiable to divide this clade into at least two genera.

Similarly, the Octadecabacters' ANI profile is distributed around a lower mean than that of the intra-genus range observed for other organisms [85] (Figure 4.7). This metric would suggest division into five distinct genera. Two of these are formed by the members of groups I and II, as between them, the nucleotide identity within their compared fragments peaks around $85 \%$ (Figure 4.7 , compare to data in ref. [85]).

However, the suitability of sequence-based measures for genus delineation, particularly that of ANI, has recently been questioned by some authors (e.g. ref. [86]). Instead, Qin et al. propose the percentage of conserved proteins ( $\mathrm{POCP}$ ) as an alternative, defining a genus as the "group of species with all pairwise POCP values higher than $50 \% "$ [86]. They consider two proteins as conserved when these show reciprocal BLAST hits above a defined score, identity, and alignment length. The orthologue detection performed here used similar criteria (minimum $35 \%$ identity, versus $40 \%$ in Qin et al., and $65 \%$ alignment coverage, 


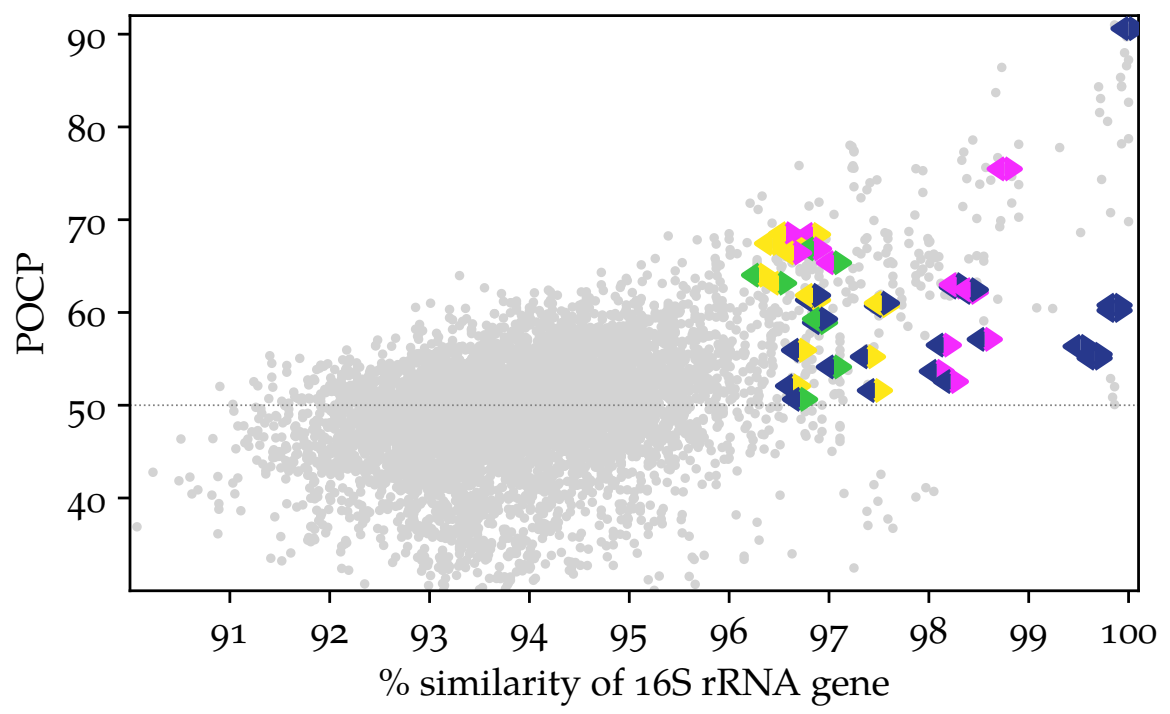

Figure 4.8: Percentage of conserved proteins (POCP) versus $16 \mathrm{~S}$ rRNA gene similarity in roseobacter strains. $\mathrm{POCP}$ in this case refers to the number of shared OGs, divided by the total number of genes in each two compared genomes. Data for Octadecabacters are represented with larger symbols. Symbol colours indicate the groups (Section 3.2), to which the two compared Octadecabacter strains belong. One proposed definition suggested that a genus comprise the set of bacterial strains in which all pairwise $\mathrm{POCP}$ are higher than $50 \%$ [86].

versus $50 \%$ [86]), so that comparison of the inferred orthology relationships should reflect the РОСР well. When applying the proposed genus definition to the current case, it becomes clear that it is not suitable for the Octadecabacters, or the Roseobacter group in general, at least not when using the stated criteria for determining conserved proteins.

The core-genome constitutes the largest fraction of all Octadecabacter strains' CDSs (see previous section), and all nine consequently show pairwise POCPs of over $50 \%$ (Figure 4.8). However, assignment of at least $P$. jejudonensis and isolate $\mathrm{NH}_{9}-\mathrm{P}_{7}$ to a different genus than the rest is strongly supported by multiple factors, including their distant clustering in the phylogeny, the low sequence similarity (Figure 3.1), and their variation in GC-content (Table 3.1), in addition to the differences observed by Billerbeck et al. The generally high POCPs reflect the coherence in genetic equipment discussed in Section 4.1.1. Notably, when compared among each other, groups II to IV show POCP values well over $60 \%$ (Figure 4.8). This again underlines the polar group's divergence concerning gene content. It also demonstrates how vulnerable an individual metric can be to the boundary conditions presented by a particular group of organisms.

Ultimately, rather than basing it on one set standard, the definition and delineation of genera should be based on multiple criteria and should follow a careful individual assessment, taking into account the specific properties of the group of organisms in question. For example, 
keeping in mind that functional features like carbon compound utilisation, nitrogen fixation, or even particle colonisation [287, 295] are phylogenetically dispersed, their distributions among bacterial strains are unsuited to define genus or species boundaries in functionally versatile organism groups with high genome flexibility.

\subsection{FUNCTIONAL GENE CONTENT AND ITS EVOLUTION}

Polar Octadecabacters show some notable differences in their functional gene content to the temperate isolates (as captured by the COG classification, Figure 3.7 p. 36). Beyond a simple group-based comparison, gene content evolution was reconstructed by means of a phylogenetic birth-and-death model, which allows for predictions of functional gene content at ancestral nodes and its development along the Octadecabacter lineage. Using COGs to that end reduces the resulting data complexity, as functionally equivalent proteins are assigned to the same family, whereas the more stringent orthology detection method discussed in the previous sections might assign them to different OGs (asserting different hereditary backgrounds, even if the actual function may be similar). However, this simplification is at the cost of reduced integrity, since only 78 to $83 \%$ of CDSs in the Octadecabacter genomes were actually assignable to a COG. Following, the general trends in COG content of polar Octadecabacters will be interpreted, including the category size changes along the Octadecabacter lineage when they are of interest (Section 4.2.1). The ecological significance of COGs gained at the polar Octadecabacter LCA and the involved pathways will be discussed in Section 4.3. Besides these results, an evaluation of the estimated parameter values and general properties of the birth-and-death model can help assess its credibility, and can reveal general trends in individual lineages. Such an evaluation will be undertaken in Section 4.2.2.

\subsubsection{General trends in COG content}

As stated earlier, polar Octadecabacters possess, on average, 800 additional CDSs in comparison to temperate isolates. These are not spread evenly among the COG categories. Genome fractions devoted to DNA metabolism and informational functions (i. e. categories J, D, F, and L) usually negatively correlate with genome size, presumably because the additional turnover generated by larger genomes can still be processed by the same number of genes in these categories [296]. This behaviour was also found in polar Octadecabacters for categories J, F, and D. Furthermore, they show little change along the Octadecabacters' ancestral lineage, judging from the probabilities assigned by the birth-and-death model (Figure 4.9).

However, COG category L (Replication, recombination, and repair) is disproportionately stronger represented in polar Octadecabacters 

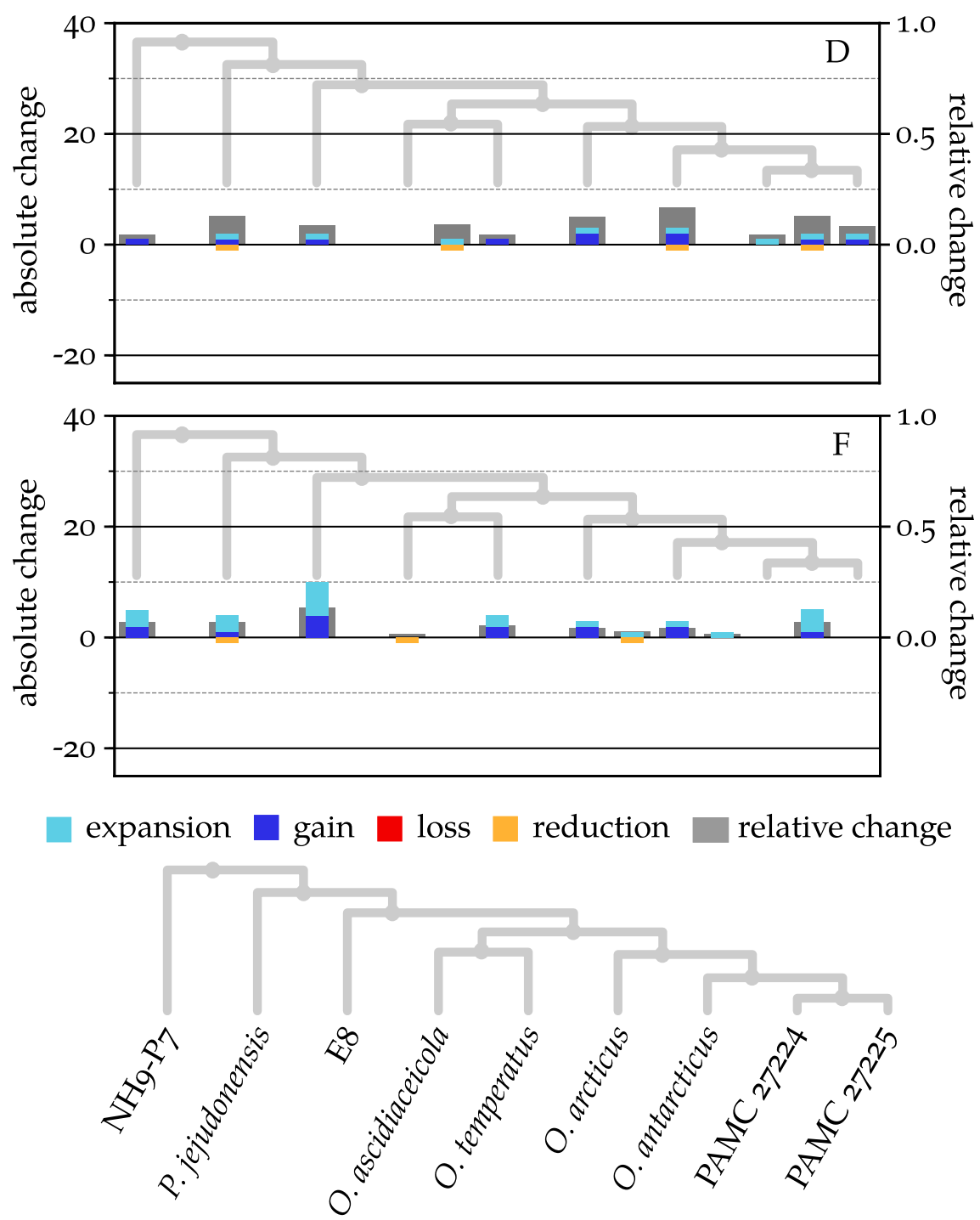

(a) Categories D and F

Figure 4.9: Changes in COG categories D (Cell cycle control, cell division, chromosome partitioning) and F (Nucleotide transport and metabolism) (a), and categories J (Translation, ribosomal structure, and biogenesis) and L (Replication, recombination, and repair) (b) in the Octadecabacter lineage. The number of all COGs in the indicated category, which were assigned a probability $p>0.5$ of either gain, loss, expansion, or reduction by the phylogenetic birth-and-death model, are given above for each node in the Octadecabacter clade phylogeny (coloured bars, left scale). This number was normalised by the total number of different COGs of that category at that node (that is, all respective COGs with a summed probability $p>0.5$ of occurring once or multiple times) to obtain the relative change, indicated by grey background bars (right scale). The overlaid cladogram indicates the order in which the comparison genomes are shown, which is based on their phylogenetic relationships. Note that the expansion and reduction bars do not represent absolute gene numbers, but only the number of COGs in which the respective change occurred. 


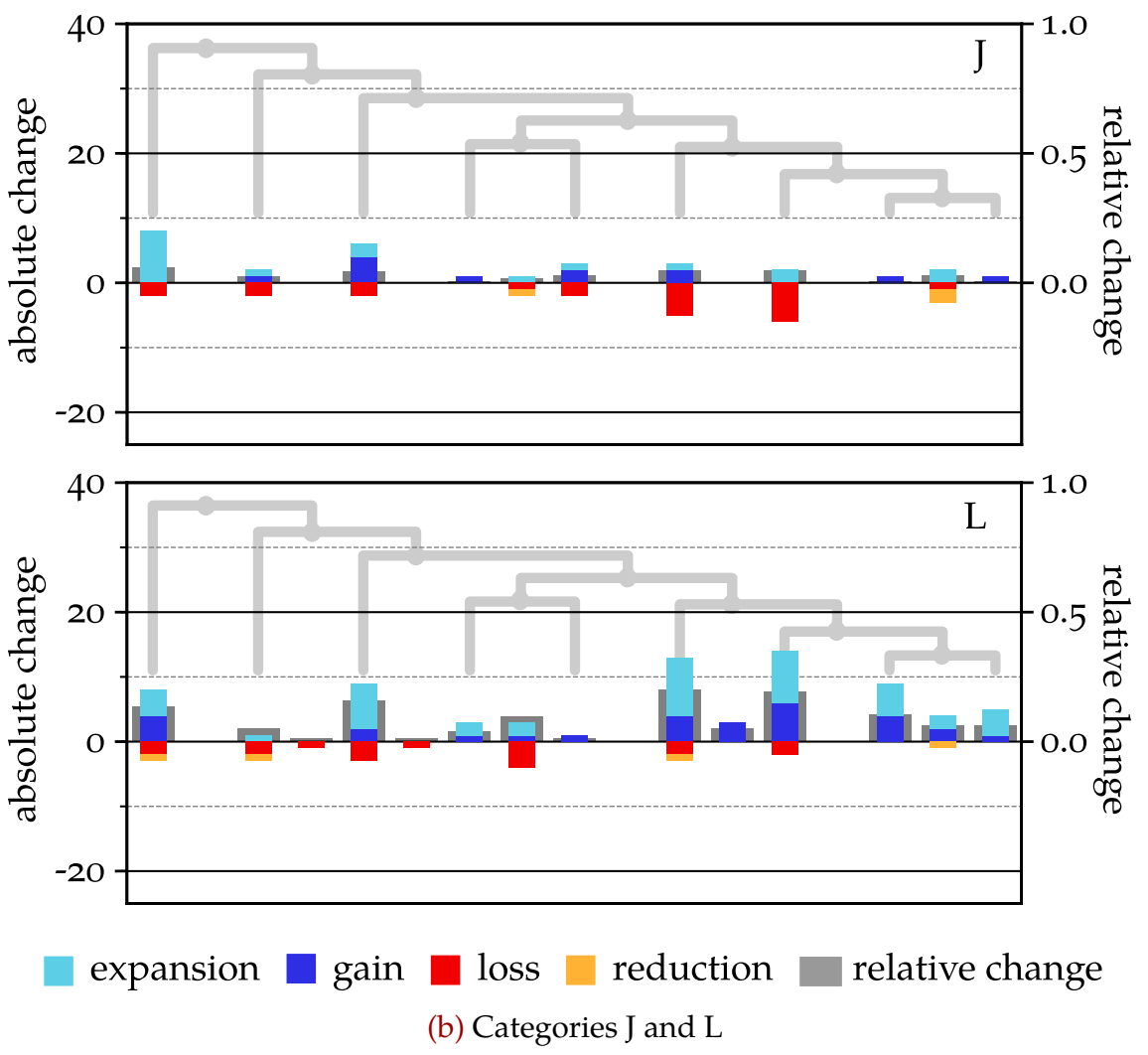

Figure 4.9: (continued)

(Figure 3.7a). Its change is strongest in these species, and most of it likely occurred after branching from their common ancestor, close to the extant taxa (Figure 4.9b). This already illustrates the importance of recombinatorial effects for environmental adaptation of these strains, which will be discussed in more depth in Section 4.3.1. Notably, DNA repair mechanisms also fall into this category, which, as mentioned in Section 3.4.1, are of particular importance in polar habitats (further discussed in Section 4.3.3).

The distribution of COG categories C (Energy production and conversion) and $\mathrm{N}$ (Motility) in Octadecabacter genomes further contrasts previous findings. Normally they would be expected to positively correlate with genome size [296], but instead they show negative correlation ${ }^{2}$ in polar Octadecabacters. Nevertheless, gain and expansion events dominated in both categories (Figure 4.10). All Octadecabacters possess annotated genes for flagellum synthesis, which constitute the majority of COG category $\mathrm{N}$ genes in their genomes. In addition, $O$. arcticus and $O$. antarcticus gained (non-paralogous, i. e. likely horizontally acquired) flagellar genes, leading to large changes in this category. Motility tests of these two strains, however, gave negative results [61]. Consequently, it remains unclear under which circumstances these two

2 term correlation as used in ref. [296], i. e. referring to occupied genome fraction versus genome size; see also Section 3.3.2 
strains show motility, and what the function of their additional flagella synthesis genes may be.

Category $\mathrm{C}$ comprises functions in energy production and conversion, and includes the xanthorhodopsin specific for polar Octadecabacters, which was likely gained by a common ancestor and then passed down vertically to subsequent generations (Table 3.5). Apart from acquisition of this new function, expansion of existing gene families in this category predominate for the extant polar representatives, which contrasts more distant clade members like isolates $\mathrm{NH}_{9}-\mathrm{P}_{7}$ and $\mathrm{E} 8$ (Figure 4.10). Drastic changes concerning energy production and conversion therefore seem to have played a lesser role in polar habitat adaptation. Rather, as Sections 3.4.1 and 3.5 demonstrated, polar Octadecabacters utilise a broader range of substrates and integrate different environmental stimuli. Accordingly, COG category G (Carbohydrate Transport and Metabolism) shows a higher representation in their genomes (Table 3.4). Nonetheless, overall restructuring in this category, i.e. the amount of function gain and loss, is high in all extant clade members (Figure 4.10), likely since they stem from diverse habitats with different nutrient spectra (consider also remarks in Section 4.1). The mobilome presents a similar case, in that all extant nodes show large relative changes in COG category $X$ (Figure 4.10), but it occupies a larger fraction in polar Octadecabacter genomes than in temperate ones (Table 3.4). This agrees with previous observations that temperate bacteria possess mobilomes which are distinct from their psychrophilic relatives, but that the latter usually contain more mobilome components, like ISs [297].

Categories $\mathrm{R}$ and $\mathrm{S}$, comprising genes with poorly characterised or wholly unknown functions, exhibit the largest changes along the Octadecabacter lineage (Figure 4.11), although they do not constitute the largest categories overall (Figure 3.7). A high fraction of uncharacterised genes was also noted for the specific genome of Octadecabacter group II (Section 3.4.2). Consequently, a large part of functional habitat adaptations seen not only in polar, but all Octadecabacter clade genomes, is currently not available for interpretation, which is a general caveat of content-based analyses (Section 1.5).

Large genomes were found to encode a higher proportion of genes associated with transcription [296]. Polar Octadecabacters do not show this trend, although multiple transcriptional regulators are part of their group-specific genome (Section 3.4.1). Overall, the variation seen in this function (COG category $\mathrm{K}$ ) along the Octadecabacters' lineage is of comparable degree between polar and temperate isolates (Figure 4.11). Co-factor synthesis (category $\mathrm{H}$ ) presents a similar case, despite the polar members' increased synthesis capabilities (Sections 3.4.1 and 3.5). Both observations reflect the fact that the group-specific genome, or functional changes at the LCA, only represent a small part of the variation in genetic equipment during habitat adaptation. Rather, the ma- 

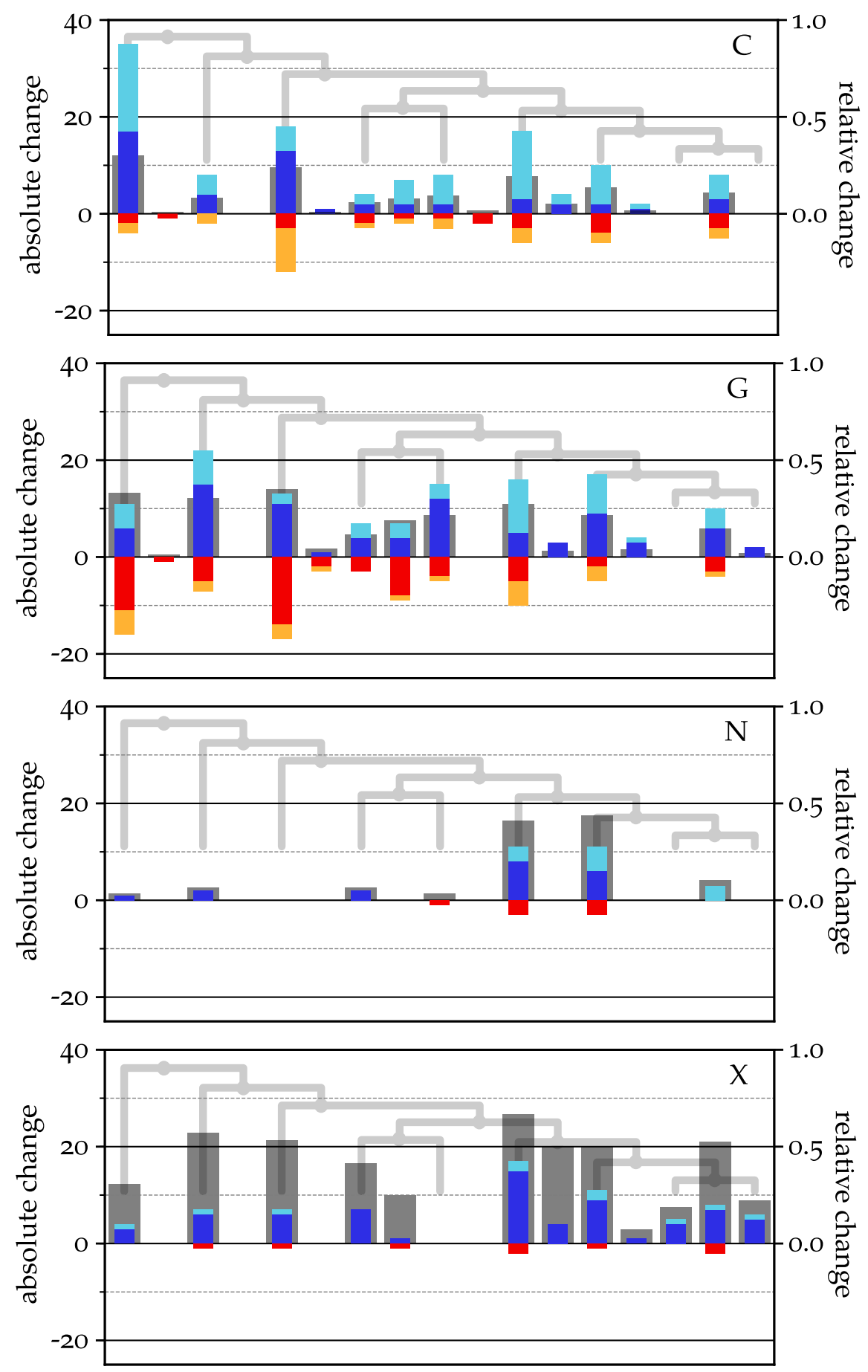

expansion $\square$ gain $\square$ loss $\square$ reduction $\square$ relative change

Figure 4.10: Changes in COG categories $C$ (Energy production and conversion), G (Carbohydrate transport and metabolism), N (Motility), and X (Mobilome) in the Octadecabacter lineage. For details, see caption of Figure 4.9. 
jority of changes occur on the level of the individual strain. However, group-specific genes and ancestral changes reflect more general aspects of the adaptation, independent of the ecological niche inhabited by individual polar species, and therefore allow for more generalised conclusions.

In summary, the differences in COG category distribution between polar and temperate Octadecabacters are partly derived from the former's larger genomes, particularly concerning functions in DNA metabolism and information processing. On the other hand, categories which show a stronger representation in polar genomes are more related to the ecology at this particular habitat (discussed in more detail in Section 4.3). Their increased capacity for nutrient uptake and degradation indicates a copiotrophic lifestyle, which would be plausible given the usually high microbial density and primary productivity in the sea ice habitat (Section 1.2). Earlier studies by Lauro et al. found that, compared to oligotrophs, copiotrophs possess significantly more genes involved in cellular motility, signal transduction, transcription, and defence mechanisms (COG categories $\mathrm{N}, \mathrm{T}, \mathrm{K}$, and V, respectively), and less genes involved in secondary metabolism, and lipid transport and metabolism (categories Q and I) [298]. Judging from these observations, polar Octadecabacters do not seem to follow a more copiotrophic lifestyle than their temperate relatives, and the implications of this situation are discussed in Section 4.3.2.

\subsubsection{Properties of the birth-and-death model}

Wolf and Koonin argue that genome reduction is the predominant process in genome evolution [299]. They suggest that genome complexity does not emerge continually, but punctually, and is followed by gradual reduction as organisms adapt to their specific niche [299]. Consistently, former ancestral reconstructions in a broad phylogenetic background estimated the Roseobacter group's LCA to have had over 8,ooo genes [93]. From this ancestor, gene counts remained high compared to the extant genomes up to relatively late bifurcations, with subsequent successive losses. According to a model computed by Luo et al., the genome size of the Octadecabacter and Loktanella clade's LCA was around 7,400 [93]. In this regard, it seems consistent that gene loss also constitutes the strongest lineage-specific component in almost all deeper branches of the model computed here (see Figure 3.19 for parameter visualisation within the Octadecabacter clade).

Luo et al. also found linear correlation between expected amino acid substitutions and gene duplication and loss rates, from which they predicted abnormally high gene duplication rates for both $O$. arcticus and O. antarcticus [93]. It is, however, only sensible to relate parameter values to the branch lengths in the phylogenetic ML-tree, if these correlate with the branch length parameter estimated by CounT, for which they 

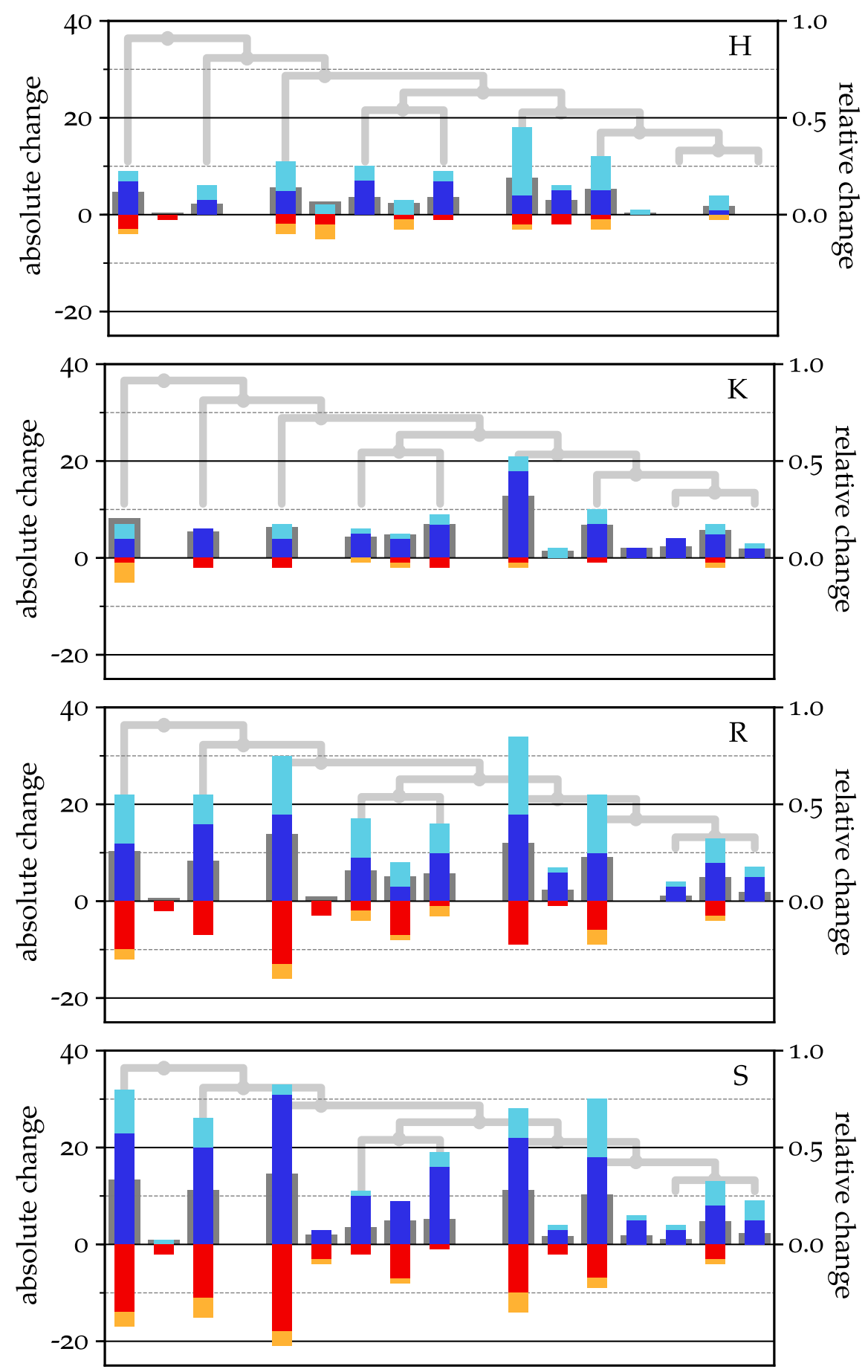

expansion $\square$ gain $\square$ loss $\square$ reduction $\square$ relative change

Figure 4.11: Changes in COG categories $\mathrm{H}$ (Coenzyme transport and metabolism), K (Transcription), R (General function prediction only), and $\mathrm{S}$ (Function unknown) in the Octadecabacter lineage. For details, see caption of Figure 4.9. 
provide no detailed information. In the present analysis, the model tends to assign relatively shorter lengths to internal edges as compared to the ML-derived lengths, and both correlate poorly $\left(R^{2}=0.09\right.$, $p=0.82$, Figure 3.19). Edges to extant species correlate well, and show a tendency towards longer branch lengths for the polar Octadecabacters in the birth-and-death model, reflecting their more extensive content-based divergence (mentioned in Section 4.1.1). Based on these data, no investigation of clock-like parameter behaviour as in ref. [93] was undertaken. Contrary to the findings by Luo et al., the parameter values at most extant and internal nodes of the polar Octadecabacters suggest that transfer, i. e. acquisition through innovation or LGT, and not duplication, has been most important in their functional adaptation. This observation agrees with the recent finding that $\approx 85 \%$ of gene family expansions are due to horizontal acquisition versus $\approx 15 \%$ to duplication [289]. Only the two PAMC isolates show high duplication values, albeit at short branch lengths. The discrepancy between this and Luo et al.'s models may result from the lower depth and higher resolution of the phylogeny used here, but also from the fact that the present model was optimised on COG data, and not on orthology data derived from pairwise BLAST comparisons.

There are no striking similarities in parameter values among the three Loktanella species, which were isolated from Antarctic lakes [55]. L. vestfoldensis DSM 16212 has high rates of transfer and duplication, paralleling to some extent the polar Octadecabacters (Figure A.7b, p. 170). L. salsilacus and L. fryxellensis, which fall into a different subclade, show no strong preference of any specific parameter. This suggests that these species may have followed different strategies in adapting to their polar habitats, which will be discussed in more detail in Section 4.3.4.

Among the other genomes, parameter values along the Ketogulonicigenium branch strongly differ from the rest, as they possess the highest branch length and show strong tendencies towards loss of functional families (visualised in Figure A.7c, p. 171). This signifies the drastic amount of change in functional gene content associated with adaptation of their terrestrial lifestyle.

\subsection{ENVIRONMENTAL ADAPTATIONS IN POLAR OCTADECABAC- TERS}

Sections 3.4 and 3.5 described characteristics of polar Octadecabacters and provided basic interpretations on their functions and roles in these organisms. This section seeks to integrate these data into a comprehensive view of how polar Octadecabacters adapted to their environment. This includes analysing and rating their basic mechanisms of adaptation (Section 4.3.1), and apparent methods to deal with higher levels of stress (Section 4.3.3). Several metabolism-related content alterations 
were found, and these are interpreted in Section 4.3.2. Finally, Section 4.3.4 compares characteristics of polar Octadecabacters to other polar isolates within the Roseobacter group and discusses possible reasons behind the observed similarities and differences.

\subsubsection{Genomic adaptation mechanisms}

Colonisation of a new habitat presents microbes with the challenge of adapting to new selective stimuli, potentially encompassing extreme environmental situations. Adaptation takes place via three mechanisms:

i changes in gene regulation, sometimes induced by genomic rearrangements, which alter regulatory networks,

ii protein family expansion resulting from LGT, or from duplication of existing genes, and

iii point mutations, which confer a selective advantage and therefore become fixed in the population.

The data presented in Sections 3.4 and 3.5 show that functions associated with these mechanisms are more abundant in polar Octadecabacters than in their temperate relatives, which is a trend often observed in psychrophilic bacteria [297].

In addition to GTAs, for which at least partial gene clusters are present in all currently sequenced Octadecabacters, the high number of transposable elements (TEs) and functions associated with recombination (Section 4.2.1) facilitates not only genomic rearrangement, but also uptake of laterally transferred genetic material [300]. In Section 3.4.1 some genetic loci were described, which likely constitute parts of regulatory networks that are specific to polar Octadecabacters. Many of the corresponding functions coded therein are not per se exclusive to this group, such as the carbohydrate $A B C$ transporters, or are represented by additional orthologues elsewhere in the genomes, e.g. the TCA cycle-associated dehydrogenases. Since these are not paralogous, but constitute individual OGs with equivalent functions, they were likely acquired horizontally in the ancestral lineage of polar Octadecabacters. This would also explain their conserved order in the four genomes. The conservation of these particular loci, despite the high rate of rearrangement in these genomes (demonstrated for O. antarcticus and O. arcticus in fig. 3 of ref. [77]), furthermore indicates that they are operons of functionally correlating genes. Consequently, LGT seems to have played a role not only in acquiring completely new functions such as the xanthorhodopsins, or DNA polymerase $\mathrm{V}$, but also in expanding functionalities already present in the genomes.

Regarding protein family expansion, LGT is markedly more frequent in situ than duplication [289]. Nonetheless, duplication allows for relatively fast adaptation within the time frame of a few generations [301]. 
Polar Octadecabacters generally seem to have undergone more stable gene duplication events than their closest relatives, as indicated by their higher average number of paralogues (see Table 3.6). This likely results from their higher number of mobile genetic elements, i. e. transposases, ISs, etc. At this point, it is worth noting that the activity of such elements is often increased in situations of stress, demonstrated for, e. g. oxidative stress [302], starvation [303], or UV-induced damage [304]. Multiple forms of stress are frequently encountered at polar habitats (and sea ice in particular, as explained in Section 1.2), and a large mobilome is advantageous to polar Octadecabacters, as it increases their evolvability under these conditions.

Various group-specific genes discussed in Section 3.4.1 are found in multiple copies within individual polar Octadecabacter genomes, e.g. DNA polymerase V, NfuA, or pyridoxamine 5'-phosphate oxidase, underlining their apparent importance for habitat adaptation. Nonetheless, there is no polar group-specific gene, which is duplicated in all four members of this group. The high fragmentation of the two PAMC isolate genomes may partially obscure their actual gene content, and therefore lead to an underestimation of their TEs and paralogue numbers (see also Appendix A.7, p. 203).

Generally, 45 to $92 \%$ of paralogues are exclusive to each strain, with the exception of the two highly similar PAMC isolates, and predominantly affect the cloud-, and shell-genomes (Table 3.6). Interestingly, isolate $\mathrm{NH}_{9}-\mathrm{P}_{7}$ possesses a number of paralogues similar to that of polar Octadecabacters, despite its lower number of TEs (Figure 3.7). The majority of its duplications also occurred in the clade's core-genome. Consequently, the number of mobile genetic elements alone is not necessarily a direct indicator for the number of duplications accumulated in a genome. A larger number of these elements will increase the capacity for short-term changes, which are stimulated by conditions of stress, as discussed above.

Notably, the results of transposition and homologous recombination events of mobile genetic elements are indistinguishable [300]. It is therefore unclear, which of these processes dominated in polar Octadecabacters.

TEs not only mediate gene duplications, but also horizontal transfer and rewiring of genetic networks [305]. In that function, they constitute one specific strategy an organism can follow in order to adapt. This tactic in some cases trades speed for efficiency, as mutations generated by TE activity may hinder the fixation of other, more beneficial mutations, e. g. point mutations in specific promoters [306].

Compared to TE-derived mutations, point mutations are a slower adaptive process, usually taking thousands of generations [301]. Their rates are increased in polar Octadecabacters, likely due to the acquisition of DNA polymerase V. As mentioned in Section 3.4.1, this enzyme 
causes a higher mutation rate while bypassing and repairing DNA damage, thereby increasing evolvability [230].

Sea ice is thought to be a "hot spot" of microbial evolution due to its high abundance of phages and lower selective pressure due to grazing [307]. No phage-associated genes were found specific for polar Octadecabacters, and it is unclear how much of their mobilome, e. g. specific transposases. or specific gene content results from acquisition of phage DNA. Transfer and uptake of DNA is however also facilitated by the other mobilome components. Importantly, the frequent encounters of stressful situations in sea ice, e.g. rapid shifts in salinity, or phases of heavy metal exposure, require and will therefore select for highly mutable genomes. Many components of the mobilome, e.g. specific transposases or DNA polymerase $\mathrm{V}$ are specifically activated during stress response. This system of reoccurring stress signals and concomitant mutagenesis constitutes another inherent reason why this habitat acts as evolutionary "hot spot".

\subsubsection{Metabolic properties of polar Octadecabacters}

Polar Octadecabacters have acquired the ability to utilise a broader range of substrates for metabolism than other members of this clade, some of which have been mentioned in the previous sections (e. g. histidine, fuculose/arabinose). More complex metabolic networks usually require more complex regulation, and both features are associated with larger genomes [296, 308], as observed for polar Octadecabacters (Table 3.1). Metabolic complexity also correlates with the high rates of genomic rearrangement present in some polar Octadecabacters [77], since in larger genomes with more regulators, natural selection for operons is relaxed [309].

Compared to the marine environments from which other Octadecabacters were isolated, this difference in metabolic capabilities likely results from sea ice being a complex, highly productive habitat [42] where microbial cell densities may reach over $10^{7}$ cells $/ \mathrm{mL}$ [310]. The sea ice residents $O$. arcticus and $O$. antarcticus therefore likely live in close association with primary-producing Diatoms, as it is common for the most abundant sea ice bacteria [311]. Such relationships usually influence the capacity for vitamin and co-factor synthesis, and the data presented throughout Sections 3.4.1 and 3.5 indicate that polar Octadecabacters act as providers of thiamine (Section 3.5.1) and cobalamin, which are frequently exchanged between prokaryotes and their algal hosts $[256,312]$. Nonetheless, when cultivated in vitro, O. antarcticus and $O$. arcticus cannot grow without vitamin supplements, indicating auxotrophy for at least one co-factor [76] (for example pantothenate, as noted in Section 3.5.6).

The fact that most degrading enzymes require co-factors for their reactions may also link their increased production capabilities to the 
broader range of degradable substrates in polar Octadecabacters. To name one example, the glucan phosphorylase activity likely gained at the polar Octadecabacter LCA (Section 3.5.6) requires pyridoxalphosphate, which all Octadecabacters can synthesise. The polar group gained an additional, group-specific pyridoxamine 5 '-phosphate oxidase for its synthesis (Section 3.4.1).

In their relation with primary producers, the associated prokaryotes gain access to degradable photosynthates in exchange for their secreted co-factors, and this relationship is the main carbon source for heterotrophic bacteria, as detailed in Section 1.1. Indicative of this relationship are the glucan phosphorylase and glucanotransferase gained by the polar Octadecabacter LCA (Section 3.5.6), as well as their increased carbohydrate transport capabilities mentioned in Section 3.4.1. Due to the high levels of primary production [42], and since EPS produced by Diatoms also serve to concentrate DOC [37], sea ice generally favours a copiotrophic lifestyle. It is generally accepted that copiotrophs possess more genomic copies of the rRNA operon [298, 313], as well as larger genomes and increased transport and degradation capabilities [298]. Polar Octadecabacters exhibit these features in comparison to the other clade members, although the higher rRNA operon copy number is masked in the PAMC isolates by their high fragmentation (but see Appendix A.7, p. 203). Nonetheless, they do not show the typical COG profiles associated with copiotrophy (Section 4.2.1). They furthermore exhibit slow growth rates even in full medium (for data on $O$. arcticus and O. antarcticus see ref. [61]), which is more typical of oligotrophs. One explanation might be that they require parts of their increased metabolic capabilities and available energy for the repair of cellular damage, at the cost of growth speed and efficiency. A higher number of rRNA and tRNA genes was also suggested to compensate for reduced translation speeds at low temperatures [39]. Notably, numerical dominance of $\alpha$-Proteobacteria, and Octadecabacters in particular, seems to be relatively uncommon in sea ice, and other known copiotrophs from the $\gamma$-Proteobacteria and Bacteroidetes usually dominate the prokaryotic communities $[53,311]$.

It could seem paradoxical that the sediment-residing PAMC isolates, which were procured from a depth of $156 \mathrm{~m}$ [80], show the same metabolic and substrate patterns as the sea ice residents $O$. arcticus and $O$. antarcticus. Studies in the Arctic showed that during melting of sea ice, algal biomass sinks to the ocean floor and can reach depths over 4,000 $\mathrm{m} \mathrm{[314].} \mathrm{Such} \mathrm{a} \mathrm{process} \mathrm{could} \mathrm{avail} \mathrm{the} \mathrm{two} \mathrm{isolates} \mathrm{the} \mathrm{same}$ nutrient spectrum as their surface-dwelling relatives.

In contrast to sea water, nitrogen is abundantly present in sea ice, and does not usually constitute a limiting factor for prokaryotic growth [53, 315]. This is reflected in the group-specific Octadecabacter genomes, since only group III possesses multiple extracellular proteases, which are secreted to make nitrogen accessible through the degradation of 
extracellular proteins. Thus, in concordance with the presence of a specific starvation-inducible factor, this group seems to frequently encounter phases of (nitrogen) starvation. On the other hand, the number of genome-encoded metalloproteases seems to have undergone a reduction in the polar Octadecabacters' ancestral lineage (Table 3.5).

Rather than nitrogen, phosphorus seems to be the most limiting element in sea ice [53]. However, it is unclear from the group-specific genome, in how far this influences the core metabolism of polar Octadecabacters. Curiously, a nucleotide phosphodiesterase likely experienced family reduction along their ancestral lineage, which would make inorganic phosphate accessible from extracellular nucleotides (Section 3.5.6). Conversely, a potentially higher amount of rRNA, implied by the increased rRNA operon copy numbers in this group, would constitute a larger sink of cellular phosphorous [316]. The presence of TauD may cause a slight alteration in their inorganic phosphate pool management (see below).

Substrate utilisation is generally controlled through the integration of environmental stimuli, and most polar group-specific loci related to degradation are preceded by genes encoding transcriptional regulators (Section 3.4.1). In addition, polar Octadecabacters possess more genes coding for enzymatic functions which steer cellular metabolism. For example, as mentioned in Section 3.5.6, GPDH regulates substrate flow between fatty acid synthesis and glycolysis, and this functionality expanded in this group. One function in this regard, which is absent in all other Octadecabacters, is the taurine dioxygenase TauD. Taurine is abundant in the environment, and serves as a nitrogen, carbon, and sulphur source [317]. All Octadecabacters are genetically able to degrade it via the $t p a / x s c$ route, which first assimilates nitrogen (tpa), and then sulphur $\left(x_{S C}\right)$ from taurine, ultimately producing acetylphosphate [317]. TauD, on the other hand, directly obtains sulphite from taurine in an oxygenolytic reaction [238]. Importantly, it also acts on a number of different substrates other than taurine [238]. In E. coli, TauD expression is induced under sulphur-limitation [238], and Rhodococcus opacus explicitly does not use the tpa/xsc pathway for sulphur assimilation from taurine, but likely uses TauD [318]. Polar Octadecabacters may follow a similar tactic, since sulphur-assimilation via TauD has the advantages of i) consuming oxygen, thereby potentially reducing the level of oxidative stress, as mentioned in Section 3.4.1, and ii) conserving phosphate in the cell, as no acetylphosphate is produced. Thus, TauD is an example of a newly acquired function in polar Octadecabacters, which acts at the intersection of multiple cellular element cycles ( $\mathrm{N}$ - and S-cycles), and may therefore serve a specific regulatory function in this group. The easy acquisition of sulphite from this abundant substance may explain why this group has no need for the methionine salvage pathway (Section 3.5.3). Most Octadecabacters, including the polar group, are furthermore genomically able to degrade the sul- 
phur compound 2,3-dihydroxypropane-1-sulfonate (DHPS), which is produced and secreted by most algae and and a known "currency" in their symbiotic relationships with roseobacters [65].

The more immediate uptake and therefore regulatory capability of the S-cycle by TauD may also be related to the increased stress levels at the polar regions. Section 3.4.1 mentioned that the oxidative state of sulphur atoms in biomolecules is particularly vulnerable to modifications by free radicals, and the products of various group-specific genes, such as NfuA, or the thiol-disulfide oxidoreductase, relate to alleviating such damage. The low molecular weight sulphur compound glutathione is highly abundant in $\alpha$-Proteobacteria and is one of the most important cellular antioxidants [319]. Even under growth conditions without the presence of external stress agents, glutathione turnover in the cell is higher than that of most co-factors [320]. With turnover potentially increasing in their more oxidative environment, polar Octadecabacters may face higher loss of sulphur species due to irreversible damage, or need to rapidly synthesise quantities of glutathione (or other antioxidative sulphur compounds) during phases of acute oxidative stress. TauD would allow for a more rapid mobilisation of sulphur from taurine, or similar molecules, than the tpa/xsC pathway, and would therefore be beneficial under such circumstances.

A further notable aspect is their phototrophic capability due to presence of a xanthorhodopsin system (Section 3.4.1). Phototrophy is frequently encountered in marine habitats, since it compensates for the general lack of nutrients in most oceanic water masses, or improves trophic efficiency $[205,251]$. Of the two known alternatives, photochemical reaction centres are the more complex one, requiring 30 to 40 gene products for functional assembly [321], and forming huge membranesituated complexes [322]. Since membrane biochemistry and proteinprotein interactions are significantly altered in cold environments [28, 323], it is plausible that the polar Octadecabacters favour the more simple (xantho)rhodopsin system, which consists of one protein and its chromophor, and requires five genes for its biosynthesis. Furthermore, the activity of photochemical reaction centres generates significant amounts of oxidative stress [252]. The higher oxygen solubility at low temperatures, and the enrichment of heavy metals at both poles [46-48] both generate high background levels of oxidative stress, which might make the additional cost of reaction centres too high. In contrast, as discussed in Section 3.4.1, rhodopsins exhibit a protective role during oxidative stress by maintaining the membrane potential during disturbances in the quinol-quinone-cycle, in addition to their potential role during starvation. Nonetheless, photochemical reaction centres associated to Roseobacter group bacteria also occur in sea ice, but at lower frequency than in the underlying sea water [90]. Exclusively favouring rhodopsin-based phototrophy therefore constitutes a characteristic of 
polar Octadecabacters (discussed in more detail in Section 4.3.4), although its function in these species remains unknown.

During phases of high primary production, degradable substrates are available in copious amounts. Their production shows high spatiotemporal variability. For example, glycerol synthesis is stimulated during phases of osmotic stress. At the same time, the sea ice can melt and release its microorganisms into the water column, thereby potentially disrupting the syntrophic associations and greatly reducing substrate availability. The group-specific genome of polar Octadecabacters contains several indicators that these strains repeatedly have to deal with such phases. For example, rhodopsin activity has been implicated as a mechanism to resist starvation [205]. In addition, cobalaminindependent methionine synthase constitutes a redundant enzymatic function without the requirement of a (costly) co-factor. Finally, as mentioned in Section 3.5.1, this group possesses more functions related to thiamine degradation and recycling, which could be related to a more efficient utilisation of available cellular compounds.

Interestingly, all Octadecabacters are also capable of anaplerotic carbon fixation through the malic enzyme route (explained in ref. [324]), which may reduce their reliance on exogenous substrates.

In conclusion, the specific genome and enriched functions of polar Octadecabacters reflect metabolic adaptations, which are consistent with our current knowledge of sea ice ecology (see above). Like other studied members from the Roseobacter group [54, 257], these strains seem to be closely associated with primary producers in a mutualistic relationship.

The present analysis cannot provide statements as to how polar Octadecabacters interact with the other abundant and usually copiotrophic prokaryotic sea ice residents. While only substrate specialisation and concomitant mutualism theoretically lead to a collective optimum of biomass [325], it remains unclear at this point whether cooperative or competitive interactions dominate the relationship among these members of the sea ice microbial community (SIMCO).

It is noteworthy that polar Octadecabacters are the only group, the specific genome of which renders a relatively complete and interpretable picture of metabolic adaptations, as the above discussion of additionally utilised substrates and environmental integration showed. This may be due to their high homogeneity (i.e. pairwise similarity) in comparison to the other groups (Figure 3.1). Furthermore, most of their specific genes are functionally characterised. Conversely, the specific genome of $O$. temperatus and $O$. ascidiaceicola contains mostly uncharacterised genes. This group would thus provide a good basis for further experimental study, as their characterisation could illuminate the lifestyle aspects which both strains share, and which seem to be poorly covered by our current knowledge. 


\subsubsection{Genomic indicators of psychrotrophic adaptations}

Cold habitats constitute a large part of earth's biosphere, and cold adaptation in bacteria has frequently been studied on the genomic level (Section 1.2). Fluidity of the cellular membrane is usually maintained at low temperatures by increasing the amount of unsaturated fatty acids, and fatty acid desaturases were identified as specifically enriched in some cold-adapted organisms [15]. This is not the case in polar Octadecabacters. They possess multiple desaturase genes, but none of these is specific to this group. The average number of desaturase genes is furthermore not higher for polar Octadecabacters than for the temperate strains.

Existing data, which were compiled by Billerbeck et al., also show that the lipid profiles of the compared clade members are relatively similar, and that the content of polyunsaturated fatty acids in temperate isolates can actually exceed that of polar ones at low temperatures (table 2 in ref. [79]). Polar strains seem to possess a slightly higher percentage of shorter fatty acids, as it is common for cold-adapted organisms (Section 1.2). Some adaptation may also derive from differential regulation of fatty acid synthesis, since polar Octadecabacters possess additional GPDH functionality (Section 3.5.6), which can steer substrates between fatty acid synthesis and glycolysis. Beyond these relatively minor alterations, polar strains seem to counter the altered membrane physiology through other means, such as an increase in degradation capabilities (indicated by the group-specific AAA ATPase).

In addition, polar Octadecabacters lack group-specific antifreeze proteins (AFPs) or cold-shock proteins (CSPs), which are proteins that usually accompany cold-adaptation in bacteria (Section 1.2). Conversely, the temperate group III possesses one specific CSP. As mentioned in Section $3 \cdot 4 \cdot 3$, CSPs are particularly important during the cold-shock response in mesophiles, where they halt translation and regulate transcription, in order for the organism to adapt to the new temperature [30]. Stenopsychrophiles like the polar Octadecabacters possess a constitutively cold-adapted transcriptional and translational machinery, and their CSPs rather serve for cold-acclimation [27]. Due to this lesser role, it is plausible that this group does not require additional CSP functionality for efficient growth. Cold-adaptation of the translational machinery in polar Octadecabacters is evidenced by the group-specific ribosomal protein RpsU (Section 3.4.1) and, as mentioned in the previous section, probably by the higher number of rRNA gene copies. For the replicative machinery, gain of a DNA replication protein (COG 1484, Table 3.5) seems to constitute one adaptive trait (further discussed in Section 4.3.4).

With regard to ice crystal formation, the lack of AFPs indicates that polar Octadecabacters rely on other methods to avoid water freezing 
and its accompanying effects. For example, they possess increased capacity for the production of proline (Section 3.5.5), and for glycerol import (Section 3.4.1), which are both effective compatible solutes. Likewise, the presence of genes encoding exopolysaccharide synthesis and export proteins suggests that all members of this group are able to produce extracellular polysaccharides (EPS) themselves, and therefore likely do not need to rely on EPS produced by Diatoms to repress the freezing point of water.

Enzymatic function is usually maintained at low temperatures via a decrease of protein stability, for which exchange of only a few amino acid residues $(<1 \%)$ can suffice [326]. Since secondary structures shape the tertiary structure of a protein, and are therefore essential for its function, it is thought that such features do not differ much between temperate proteins and their cold-adapted homologues [29]. This seems to hold true for the core-proteome of Octadecabacters. Concerning relative secondary structure content, polar strains only form a distinguished group when both structural features, $\alpha$-helix and $\beta$-sheet, are considered in combination (Figure 3.21). This clustering may simply result from the fact that they are more similar to each other on the sequence level than to the rest (Figures 3.1 and 4.7). Overall, for them there is clearly no significant trend towards a higher, or lower structural feature content (Figure 3.22).

Observed differences in amino acid frequencies between psychrophilic and mesophilic microbes can vary largely, depending on the organism's class, GC-content, or the phylogenetic distance to and nature of the mesophilic comparison organisms [27, 35, 86, 327]. Particularly GC-content is a strong determinant of amino acid profiles [328], which is why $\mathrm{P}$. jejudonensis and isolate $\mathrm{NH}_{9}-\mathrm{P}_{7}$ were excluded from the comparison of amino acid frequencies within the Octadecabacter clade (Table 3.1). When compared to the remaining three temperate strains, polar Octadecabacters show only slight deviations in amino acid frequencies (Figure 3.23). Typical observations, such as reduced arginine content [35], or increase in glycine, and decrease in proline content [28] in psychrophiles, are not, or only weakly (glycine) evident in this group when the whole core-proteome is considered. However, they show higher frequencies of some hydrophobic amino acids (four out of six, Figure 3.23), a trend which is also present in other psychrophiles [35]. Moreover, these differences reflect to some extent correlations between amino acid content and optimum growth temperature noted for some model proteins. Specifically, the amino acids which occur more frequently in polar Octadecabacters tend to be less hydrophobic than the others within the same group (compare data compiled in table 2 of ref. [329]), and hydrophobicity was found to positively correlate with growth temperature [329]. The average molecular weight presents a similar case, with cold-adapted enzymes preferentially incorporating lighter amino acids [329]. In polar Octadecabacters, this trend is less 
pronounced than the decrease in average hydrophobicity. Yet, it is still notable, particularly in the group of hydrophobic amino acids, where the frequency of light amino acids, glycine and alanine, is higher in polar strains than in the temperate ones. Thus, the differences in amino acid frequencies between polar and temperate Octadecabacters are broadly consistent with general trends in cold-adapted enzymes, but agree less with data from direct comparisons of other psychrophilic and temperate organisms.

The frequency variation of an individual amino acid, which accompanies a protein's stability reduction, can be interpreted on the basis of its flexibility, and accordingly its rigidity, as captured by atomic displacement parameters, or B-factors [330]. Overall amino acid frequencies in polar versus temperate Octadecabacters do not display a tendency towards higher flexibility, judging from their B-factors as determined by Smith et al. [330] and compiled in ref [29] (see Figure 3.23). Conversely, such a tendency was shown for psychrophilic enzymes from other organisms [331]. However, $\alpha$-helices of polar Octadecabacters show a consistent trend of avoiding rigid amino acids (I, M, and A), and increasing their content of more flexible ones (mostly D, E, see Figure 3.24). Organisms can further modulate helix stability particularly through the modification of charged amino acid numbers at the helix caps [29]. A breakdown of amino acid frequency by individual sub-domains and structural sub-features is beyond the scope of this analysis, but the fact that both negatively charged amino acids (and one out of three positively charged ones) deviate in frequency in polar $\alpha$-helices (Figure 3.24) could imply the presence of this type of modification. Thus, indications are strong that polar Octadecabacters selectively reduce stability of their $\alpha$-helices.

Rather than modifying their existing enzymes, bacteria often acquire isozymes, which function better at lower temperatures, as part of their cold-adaptation strategy $[332,333]$. Some of the group-specific genes described in Section 3.4.1 are homologous versions of functions contained in the Octadecabacter core-genome, such as myo-inositol dehydrogenase, or the cluster of specific dehydrogenases. These could well constitute cold-adapted enzymes, which polar Octadecabacters acquired through lateral transfer as alternatives to those coded in their core-genome. However, due to lack of experimental data, it cannot be ruled out that they simply integrate different environmental stimuli as part of these strains' expanded metabolic networks, or serve both of these described purposes. Nevertheless, the higher capacity of polar Octadecabacters for lateral acquisition of genes, or, more generally, their high genome flexibility discussed in Section 4.3.1, thereby also constitutes a prerequisite for efficient cold-adaptation. Similar observations were also made for Photobacterium profundum, where inactivation of transposases lead to a cold-sensitive phenotype [334]. 
Beside the traits of cold adaptation discussed so far, resistance to (oxidative) stress is prominently featured in the polar group-specific genome and in this group's expanded functionality (Sections 3.4.1 and $3 \cdot 5 \cdot 5)$.

Some of the involved genes are part of the SOS-response. As mentioned in Section 3.5.5, this response is induced by DNA damage and leads to expression of multiple genes, mediated by the cleavage of the transcriptional repressor LexA [276]. The precise number and nature of genes under LexA-regulation varies between taxa, but its core-regulons in different bacterial groups usually include the lexA gene itself, transposases, helicases (such as RuvABC), and DNA polymerases capable of translesion DNA synthesis (TLS) [276]. Polar Octadecabacters possess more copies of LexA, transposases, and a specific copy of DNA polymerase $\mathrm{V}$, and therefore show increased functionality to cope with DNA damage, as conveyed by the SOS-response. The higher number of lexA genes could mean a higher base expression, or a more rapid induction of this repressor, and may be particularly important for the survival of this group when one considers the function of DNA polymerase V: this enzyme, in addition to mediating TLS, acts as DNA damage checkpoint and can halt DNA synthesis, depending on the state of the UmuDC complex [335]. The latter function leads to a cold-sensitive phenotype in $E$. coli when UmuDC levels become too high [336]. Thus, both to prevent the rapid accumulation of random mutations, as well as to enable cell cycle progression, UmuDC expression has to be tightly controlled in polar Octadecabacters. Moreover, this control has to take place against high background levels of DNA-damaging agents (Section 1.2), which may necessitate more of the transcriptional repressor LexA.

Increased levels of (oxidative) stress also lead to more unwanted modifications of metabolites, which the cell has to deal with. As noted in Section 1.5, we currently only have rudimentary understanding of this particular aspect of metabolism. Nonetheless, some of the polar Octadecabacters' characteristics are likely associated with this effect. For example, the high number of paralogues in their genomes can be useful to deal with metabolites that are chemical variants of normal compounds [95], e. g. irregular variants derived from (unwanted) radical reactions. On the other hand, due to the high diversity and productivity of their environment, this could also simply result from a high substrate variation in their habitat. It would be interesting to experimentally test and compare, which of these purposes outweighs the other, both in the polar group, as well as isolate $\mathrm{NH}_{9}-\mathrm{P}_{7}$, which shows similarly high paralogue numbers (Table 3.6).

The modification of sulphur species in oxidative reactions, and the concomitant increase in polar Octadecabacters' respective damage protection and repair capabilities was detailed at multiple places in Chapter 3. At the same time, it is of equal importance to guarantee the integrity of other cellular compounds such as nucleotides, or co- 
factors. Nudix hydrolases are often involved in quality control of the former [95], and this functional family expanded at the polar Octadecabacters' LCA (COG 1051, Section 3.5.5). Involvement of this family in dealing with oxidatively modified compounds could also explain their role in heavy metal resistance detailed in Section 3.5.5. Besides this example, multiple uncharacterised oxidoreductases were likely gained in the ancestral lineage of polar Octadecabacters (Table 3.5), and such enzymes are often part of mechanisms which repair irregular chemical modifications of molecules [95].

Recent experimental data indicate that the rate by which most cofactors are synthesised is adjusted to account for the dilution resulting from cell division [320]. Conversely, this dilution accounts for most of the co-factors' turnover, as cells minimise their loss due to unwanted and damaging (e.g. oxidative) reactions via specialised repair mechanisms [320, 337]. The fact that the cultivated polar Octadecabacter species have long doubling times implies a decreased turnover of most co-factors in these organisms and a concomitantly higher importance of their repair mechanisms. Importantly, organisms counter co-factor damage by degrading and re-synthesising them from their scavenged components. Apart from the metabolic advantage of potentially conserving nutrients, the additional capacity for thiamine synthesis and degradation present in polar Octadecabacters (Section 3.5.1) may therefore also play a role in their management of damage to this compound through unwanted modifications. This may also apply to the alterations in their biosynthesis machinery of other co-factors, detailed e.g. in Sections 3.4.1 and 3.5.2. Yet, without experimental verification, it remains unclear to what extent these relate to guaranteeing the proper chemical nature of the respective co-factor, or to their generally changed biosynthesis patterns due to different syntrophic relationships discussed in the previous section.

It was noted earlier that besides passively reacting to oxidative stress through the mechanisms described in the preceding paragraphs, polar Octadecabacters also possess more enzymatic functions which consume oxygen, and could thus actively eliminate an important source of this type of stress. Interestingly, epiphytic bacteria on a sea ice $D i$ atom were shown to reduce its oxidative stress levels resulting from photosynthesis [54]. Among these bacteria were Sulfitobacter species, which belong to the Roseobacter group. Since polar Octadecabacters also seem to be closely associated with sea ice primary producers, they may plausibly be involved in the same kind of relationship, and this would be interesting to test experimentally.

One particular feature of sea ice is its high potential for osmotic stress (Section 1.2). The specific genetic equipment of polar Octadecabacters contains several ion transporters, mostly $\mathrm{Na}^{+} / \mathrm{H}^{+}$antiporters, which are implicated in osmoregulation (Sections 3.4.1 and 3.5.5). Importantly, such transporters are also frequently associated with cold- 
adaptation [39, 338]. As mentioned in Section 1.2, cells may increase their cytoplasmic ion concentrations to suppress the freezing point of water, and these transporters may therefore play a role in maintaining the balance between ion influx and export. It was also noted that the polar Octadecabacters' transport capability for the common polyamines spermidine and putrescine is increased as well (Section 3.4.1). Apart from acting as compatible solutes, i. e. osmoprotectants, they also constitute nitrogen reservoirs and protect DNA against oxidative damage. Despite this range of desirable functions, and the likely absence of Nitrogen-limitation (see previous section), polar Octadecabacters cannot synthesise these compounds on their own. This may be due to the fact that spermidine is toxic at low temperatures, likely by inhibiting ribosomal activity [339]. Rather, as mentioned earlier, they seem to prefer proline and glycerol as compatible solutes.

\subsubsection{Comparison to other polar members of the Roseobacter group}

Roseobacter group bacteria seem to be well represented in most polar aquatic environments, such as polar waters [89], sea ice [51, 90], Antarctic lakes [55], or marine sediment [80]. Comparison of key features between polar and temperate isolates of three clades other than the Octadecabacters indicated that the trends observed in the latter are not common in polar-adapted roseobacters (Figure 3.20). More precisely, only polar Loktanella species showed a potentially increased capacity for genetic mobility (i.e. more genes in COG categories L and X), and above-median numbers of singleton genes and paralogues, as found to be characteristic for polar Octadecabacters. The differences in these key characteristics between polar and temperate clade members are smaller for the Loktanella clade compared to the Octadecabacters. The single polar isolates associated with the Leisingera and Sulfitobacter genera, respectively, did not display these trends at all (Figure 3.20).

Consequently, it seems that large-scale increase in genomic flexibility as an adaptive strategy to polar habitats is a characteristic of the Octadecabacter clade, and polar members of other clades follow other routes of adaptation. This increase is in agreement with general observations regarding sea ice habitats [307], but can also be observed in the sediment-residing PAMC isolates, indicating that it is not specific for one particular type of polar habitat, but rather for this group of organisms as a whole. The polar Loktanella strains, on the other hand, follow a markedly different lifestyle (i.e. bio-mat association), which may require other evolutionary strategies for efficient adaptation.

The latter were also included in the phylogenetic birth-and-death model, which enabled a comparison of gain and loss of specific genes along both their and the polar Octadecabacters' ancestral lineages (Section 3.5). Two functions were specifically acquired along all polar lineages: COG 1484 (DNA replication protein DnaC), and COG 2608 (Copper 
chaperone CopZ). CopZ is a copper-efflux chaperone, and therefore plays an important role for the cell in reducing oxidative stress [264]. DnaC acts as a regulator of the DNA helicase DnaB, and is essential during replication initiation [340]. While the former is clearly involved in a function that is of higher importance in cold than in temperate habitats, the meaning behind the gain of DnaC in a polar context remains enigmatic. More precisely, due to its essential function, all comparison strains possess an annotated version of it. However, the respective gene shows conflicting orthology relationships: one orthologous group (OG) shared by all is annotated as DnaC in some strains, and as DnaB in others, whereas it is assigned to COG 0305 (Replicative DNA helicase). COG 1484, on the other hand, occurs as multiple, mostly singleton, OGs in the polar isolate genomes, and was assigned as either DNA replication protein, or as an insertion sequence (IS)-associated protein. Consequently, the current unknown functions and relationships of these genes bar further insight at this point, but they could be interesting candidates to study their function and potential role in psychrotrophic adaptation in vivo.

As noted in Section 3.4.1, rhodopsin phototrophy seems to be frequent in polar Roseobacter group isolates, and it constitutes the only type of phototrophy found in polar Octadecabacters (Section 4.3.2). Interestingly, among polar roseobacters, which are not members of the Octadecabacter clade, the genes required for xanthorhodopsin synthesis and aerobic anoxygenic photosynthesis (AAP) are equally abundant (present in 3 out of 5 isolates). Loktanella vestfoldensis DSM 16212 and Sulfitobacter guttiformis KCTC 32187 are genetically capable of both AAP, and xanthorhodopsin phototrophy. On the other hand, the two polar type strains of $L$. fryxellensis and L. salsilacus each only possess genes for one of the two: AAP for the former, and xanthorhodopsin for the latter. Phototrophic functions are absent in Pseudophaeobacter arcticus. This seems to indicate that the role phototrophy plays for polar Octadecabacters differs from that of most other polar Roseobacter group bacteria. Notably, the different micro-environments in which other polar roseobacters dwell pose other restrictions and boundary conditions, most notably the diverse ecosystems of the Antarctic lakes [341]. Thus, in some environments, the additional cost of AAP may become feasible, whereas the benefit conveyed by rhodopsin phototrophy may be marginalised. Similarly, metabolic networks and general survival strategies vary between polar representatives of different clades, which is not feasible to investigate without more sequenced genomes of polar isolates.

Following this thought, it should be noted that the present comparison has important limitations: first, the number of other polar isolates is too low to deduce definitive trends between them and temperate representatives of their associated genera. Owed to that, it is, secondly, not clear whether different polar species form monophyletic clades 
within these genera like the polar Octadecabacters do. The two polar type strains of L. fryxellensis and L. salsilacus, for example, clearly do not, despite their close relation to each other, and L. vestfoldensis DSM 16212 falls into a different sub-clade altogether (Figure 3.2). Therefore, these three isolates do not form a distinct group like the polar Octadecabacters, and may simply be derived from independent allopatric speciation events, showing spatially limited distributions and concomitantly smaller population sizes [342]. Such differences in evolutionary background and general ecology reduce the explanatory power of a comparison between both groups.

Nonetheless, these limitations also underline the Octadecabacter clade's current unique position among the roseobacters: the fact that its polar isolates do indeed form a common sub-clade, which contains comparatively many sequenced representatives, makes this group the most feasible to study adaptations to polar habitats. Furthermore, the two type strains of $O$. arcticus and $O$. antarcticus are stenopsychrophilic, meaning that they require temperatures for growth below $\approx 16^{\circ} \mathrm{C}$. All other included polar roseobacter isolates are merely eurypsychrophilic, which means they are able to grow above $16^{\circ} \mathrm{C}$, and show higher optimum growth temperatures. Given more polar isolates throughout other Roseobacter group clades, it would be interesting to see how stenopsychrophily is distributed as a trait, and how steno- and eurypsychrophilic adaptations differ within this group of organisms.

\subsection{GLOBAL Distribution OF OCTAdECABACTER-RELATED SE- QUENCES}

While the environment, from which an individual Octadecabacter strain was isolated, may give an indication of its preferred lifestyle, this thesis sought to more closely investigate biome preferences and the global distribution of Octadecabacters by means of metagenomic read mapping. Indeed, looking at different Octadecabacter groups, some habitat preferences became apparent (Section 3.9). For example, the highest frequencies of mapped nucleotides observed for groups I, II, and III occurred at high, intermediate, and low latitudes, respectively. Group I was preferentially detected in waters of the North Atlantic Ocean, and group II showed closer association with coral reefs, algal blooms, and coastal waters in general (Figures 3.26b and 3.27). Within the Octadecabacters, the observations made for group II most closely mirror the habitats with the highest abundance of roseobacters in general [24], whereas the polar group's distribution resembles that of a cosmopolitan roseobacter phylotype found at the poles and the North Sea [22]. Notably, most of the metagenomes used in this analysis were sampled around the North Sea, as well as the Pacific and Atlantic coasts of North America (Figure 3.25). The southern hemisphere, on 
the other hand, is represented by less samples, which will influence the perceived distribution patterns.

Regardless, metagenomes world-wide contain sequences which are highly similar to Octadecabacters, particularly to groups I and III (Figure 3.25). Not all of these metagenomes likely contain sequences which are truly derived from Octadecabacters, because certain effects lead to false positives in both the prediction method of mg-RAST and the mapping approach used here. mg-RAST assigns reads to taxa by their highest BLAST-based similarity, which assumes that an aligned hit belongs to a close phylogenetic neighbour of the respective taxon. This assumption is not valid for reads stemming from rare genes [343], and these will therefore lead to false detection events. The mapping approach tried to contain this effect by only reporting alignments with $>90 \%$ identity, and consequently did not return hits in all of the candidate metagenomes (Figure 3.27). Nonetheless, some bodily fluid and faeces metagenomes contained sequences with high similarity to Octadecabacters, which led to their detection in these habitats. Although these detection events are very few compared to the predictions made by mg-RAST, this precaution evidently cannot prevent false hits from influencing the perceived habitat preferences of Octadecabacter groups. False detection events may contribute to the observation that polar Octadecabacters are the most abundant and widely spread group, since they possess large shell-genomes (Section 4.1.2), and potentially contain the most rare genes within the clade. The influence of this effect on the observed distribution patterns is currently unclear. An investigation of the global distribution and abundance of (ideally fulllength) rRNA sequences related to Octadecabacters could complement the present analysis, and allow for a more wholesome view. However, such an examination is beyond the focus of this thesis.

Despite the noted limitation, observations made here suggest a cosmopolitan distribution of Octadecabacter group I, and correspond well to an already known phylotype, as mentioned above. This could correlate with their high evolvability (Section 4.3.1), in the same way that occupation of various ecological niches in the Roseobacter group is attributed to their genomic flexibility [69]. More precisely, the polar Octadecabacters' high mutagenic potential could only partly have resulted from adaptation to their extreme habitat, and may rather constitute a predisposition of this particular sub-clade, which facilitated both their cold-adaptation and cosmopolitan distribution (see Sections 4.3.3 and 4.3.4 for additional details). As a consequence, the genus associated with this group (see Section 4.1.3) may also contain non-polar, or mesophilic species which share some of the polar Octadecabacters' characteristics when compared to other roseobacters, e.g. a larger mobilome. Vollmers already noted an indication for this in an analysis of $16 \mathrm{~S}$ rRNA gene sequences, where some sequences from non-polar isolates clustered together with those of O. arcticus and O. antarcticus [61]. 
There may thus also exist an alternative means of genetic exchange to the one proposed by Vollmers: he argued that the high degree of similarity between both genomes despite their bipolar distribution indicates some form of genetic exchange, for which he proposed deepsea water currents, which could transport members of both species across tropical regions, which would normally be too warm for both to survive [61]. Stable exchange of genetic material would however also be favourable between polar members of this genus and highly similar, but not stenopsychrophilic species, the latter of which could distribute this material more widely. This would lead to an indirect genetic exchange between both poles via several closely related intermediate organisms, rather than a direct exchange through transport of the stenopsychrophilic species themselves. However, it should also be noted that the pairwise sequence similarity observed between the polar Octadecabacter strains is typical for what would be expected for members of a genus (curve I in Figure 4.7), and may therefore not be particularly special at all. It could simply imply that arctic and Antarctic species divided from their common ancestor a relatively short time ago. High sequence similarity in places such as the rRNA operon can also result from functional restraint due to the low temperature and high stress levels, restricting the number of viable mutations.

Concerning global distribution, the mapping exposed a converse situation for Octadecabacter group III: this group with two mesophilic members was the only one detected in one of the northern-most sampled metagenomes (Figure 3.25), which does not necessarily result from unspecific mapping events. Rather, it could be a further example of polar habitat colonisation, which has occurred in single lineages throughout multiple clades of the Roseobacter group (see also Section 4.3.4).

The frequencies, in which nucleotides mapped to different Octadecabacter groups, vary largely between metagenomes (Figure 3.26). They should nonetheless not be interpreted as a direct indicator of the groups' abundances, for the two following reasons: first, the methodical approach taken here underestimates Octadecabacter content in all metagenomes, since rRNA genes and regions with high sequence identity between roseobacters were excluded from the mapping. This is because due to the high pairwise sequence similarity ( $>96 \%$ for rRNA genes, see Figure 4.8), and the usually short length of NGS reads, sequences mapped to these regions could not meaningfully distinguish between different Octadecabacters, and would thus scramble the results. Their exclusion therefore increases the reliability of group assignment, at the expense of detection sensitivity.

Secondly, the pre-selection step only included metagenomes in which mg-RAST had taxonomically assigned a percentage of reads to the Octadecabacter genus (Section 2.12.1). This assignment only uses publicly available genome information, and was hence restricted to the three 
species O. antarcticus, O. arcticus, and O. temperatus, as none of the other genomes were public at the time. The metagenomes in the present analysis are therefore inherently biased towards samples, in which polar Octadecabacters and temperate species of group II occur more frequently. The fact that group IV exhibits the lowest frequencies of mapped nucleotides likely results in part from this bias, especially as it contains only one strain. Its distribution and habitat preferences are thus harder to derive from the present selection of metagenomes than those of the other groups. Nonetheless, it was noted that it featured more prominently in a number of estuarine habitats and sediment samples (Section 3.9), which could be indicative of its preferred ecological niches.

The metagenomic read mapping performed here intended to look for global occurrence patterns within the Octadecabacter clade. As mentioned above, an exhaustive analysis including estimations of Octadecabacter abundances, and correlations to specific environmental parameters, was not in the scope of this thesis. Nonetheless, it became obvious that the different sub-groups within this clade show distinct distributions. This observation lends ecological significance to the subgroup definition, which was originally purely based on phylogenetic relations within the clade and on the similarity of several genome characteristics (Section 3.2). A more detailed view of the different Octadecabacter groups' habitat preferences, and how these correlate with the group-specific genome, will be an interesting prospect of future investigations. 
The genus Octadecabacter originally consisted of only two described and genome-sequenced members, which were both isolated from sea ice. They were considered as "extreme cases" among the Roseobacter group concerning their unusually large number of transposable elements, inferred gene duplications, and genome rearrangements. However, due to the lack of directly comparable genome sequences from closely related strains, it remained unclear to what extent these observations are actual evidence of environmental adaptations in the two strains, and how these polar isolates might differ from temperate strains of the same genus on the genome level.

The present thesis expanded the number of available comparison genomes and re-examined genomic characteristics within this genus, with a specific focus on adaptations to life in polar habitats.

A direct result of this examination is the distinction between the genus Octadecabacter and the Octadecabacter clade. The latter consists of nine sequenced isolates likely associated with five different genera. Uniquely among roseobacters, four of these isolates stem from extreme polar habitats. They belong to three distinct species, which form a common phylogenetic sub-clade that arguably represents the genus Octadecabacter sensu stricto, as it includes the two species which were the basis of this genus' original definition. In combination, their specific genomic functions and genome characteristics reflect the altered nutrient spectra and stress levels encountered at their respective polar habitat, and are broadly consistent with our current understanding of sea ice ecology, and psychrotrophic adaptations described in other organisms.

Divergence in both gene content and sequence similarity within the Octadecabacter clade is similar to other clades in the Roseobacter group, which is likely due to the fact that different species within a clade often originate from different micro-environments.

A high amount of transposable elements and genome reshuffling, originally observed in $\mathrm{O}$. arcticus and O. antarcticus, turned out to be characteristic for polar Octadecabacters in general. The analyses presented here could further generalise these findings, concluding that functions related to all aspects of evolvability are more abundant in polar than in temperate Octadecabacters, and that this evolvability is an important prerequisite for polar habitat adaptation. Conversely, further comparisons among a larger set of roseobacter genome sequences now illustrated that several other Roseobacter group members, most of which do not originate from polar habitats, possess similar, or even 
higher numbers of transposable elements and unique genes, displacing polar Octadecabacters from their position as extreme cases. This indicates that genome flexibility, while playing an important role in the polar Octadecabacters' evolutionary history, does not seem to be exclusively associated with cold-adaptation in roseobacters. At the same time, adaptation to cold habitats does not generally seem to require higher potential for evolvability, as polar isolates from other clades in the Roseobacter group do not exhibit the same characteristics as polar Octadecabacters when compared to their temperate relatives. Polar Octadecabacters thus demonstrate a unique evolutionary path in their environmental adaptation among the currently sequenced roseobacters. This thesis did its best to outline this path based on all currently available genome information. In doing so, it revealed several targets which merit further investigation. For example, for several genes, which were identified as specifically gained or enriched in polar Octadecabacters, the scientific literature did not allow a reasonable prediction of their roles in environmental adaptation. Conversely, some genes were determined to have multiple possible functions by which they would contribute to the habitat adaptation of polar Octadecabacters. Such examples would be good candidates for future experiments to try and clarify their respective roles, as it would further our understanding of the mechanisms behind specific environmental adaptations. It is also noteworthy that the available polar Octadecabacter strains are good candidates for such studies, as they are readily cultivable in a laboratory setting.

Using the example of the nine currently available Octadecabacter genome sequences, the present work also demonstrated how individual metrics and methods of genus delineation are influenced by particular features of a group of organisms. In the Roseobacter group, despite their functional divergence, members of a clade typically display a coherence in genetic equipment which goes beyond this clade's core-genome, influencing the way in which delineation of genera is meaningfully possible. From a combination of different metrics, it was concluded that the Octadecabacter clade currently comprises five distinct genera, one of which is formed by the polar (including both Arctic and Antarctic) isolates. However, comparisons with metagenome data sets indicated that this polar Octadecabacter genus may be cosmopolitan, and therefore also contain species from non-polar habitats, which still remain to be isolated. This could also add a novel perspective to the observation that Arctic and Antarctic isolates show high levels of similarity in sequence and gene content despite their vast geographical separation. The mutual genetic exchange required to maintain this degree of similarity may not result from transport of individuals between poles through cold deep water streams, as an early theory surmised [61]. Rather, frequent genetic exchange may occur between exclusively polar Octadecabacters and their more widely distributed 
close relatives, the latter mediating genetic information between communities of both poles.

The present work offered a detailed account of the nature of, and possible mechanisms behind, adaptation processes to extreme habitats in some members of the Roseobacter group. As such, it serves as a basis for future analyses of similar cases within other roseobacter clades, and for elucidating and modelling adaptation tactics utilised by bacteria in general. By expanding the comparison data set with additional Octadecabacter clade genomes and other novel roseobacter isolates, the analyses presented here yielded a much improved description of this clade than previous studies could, as these were based on only two Octadecabacter isolates. Of course, it can be assumed that a higher number of closely related comparison genomes will further increase the detail and reliability of such descriptions. Therefore, it will be interesting to see this picture evolve, as further genome sequences of the roseobacter group, and possibly the Octadecabacter clade, will no doubt become available in the near future, either through improved isolation and cultivation techniques, or by metagenomic binning, or single cell sorting and sequencing approaches. 



\section{APPENDIX}

\section{A.1 SUPPLEMENTARY FILES}

The supplementary data include the output files of most computations described in Chapter 2. Most visualisations provided throughout the thesis are derived from data contained in these files. Figure A.1 gives an overview of the folder structure and the contained files.

Dissertation_Florian_Lenk_2020.pdf

\section{2_Assembly_Annotation_Statistics}

$\triangle$ Genome_statistics.tsv for all compared roseobacter genomes

CheckM_results_0ctadecabacters.tsv

2.3_Sequence_Comparison Original output files of GGDC

ggdc_results_<strain1>

ggdc_results_<strain2 $>$

.

2.5_Orthologue_Detection .poff output file from ProteinOrtho

2.6_Roseobacter_Group_Phylogeny

Core-Genome_Reduced_Gene_Content_Clustering 1 newick tree Gene_Content_Clustering 1 newick tree

MLSA RAxML output files

IC_and_TC_Scores

- ic_and_tc_values_to_reference RAxML output files

raxml_besttrees core-gene trees in newick-format

$\rightarrow$ RAxML_bestTree.unaligned_temp_fasta_OG00001.fasta

RAxML_bestTree.unaligned_temp_fasta_0G00002. fasta $\cdots$

raxml_info_files $R A x M L$ info for each tree computation

$\cdots$

Figure A.1: Supplementary file folder structure and contents (continued on next page). 


\section{7_0ctadecabacter_Clade_Phylogeny}

core-gene_phylogenies $R A x M L$ bestTree and info for each core-gene

Consensus-cluster-network.nexml network from core-gene phylogenies

\section{9_Birth-and-Death_Model}

$\checkmark$ COG_pattern.tsv COG counts for the relevant organisms

parameter_estimation_<01-04> ML-estimates of increasing complexity

Phylogeny.nex basis for model optimisation

Posterior_probabilities.tsv posterior probabilities estimated by CounT

\subsection{2_Group-specific_Genes}

results_iQ.9_eQ.1 original output of po2group_stats.pl

group_file.tsv groupdefinitions

grpstats_output.txt summary report of po2group_stats.pl

orthology_information.tsv sub-frame of orthology table given in 2.5

2.11_Protein_Secondary_Structure one prediction file per annotated protein

\subsection{2_Metagenome_Mapping}

ANI_filtering BLAST-output from pyANI

bam_files all mapping results

- Mapped_frequencies_groups.csv frequencies after filtering

Mapped_frequencies_strains.tsv

Metagenome_data.csv metagenome metadata

\subsection{1_Phylogeny RAxML output of RF distance calculations}

4.1.3_Genus_Delineation dnadiff results with pairwise $16 S$ rRNA similarities Figure A.1: Supplementary file folder structure and contents (continued) 


\section{A.2 ORGANISM METADATA}

Table A.1 lists metadata to all strains used for comparative analysis, which were either acquired from the genome data source, or the reference publications given in Table 2.2. Coordinates were either provided directly, or inferred, if the names of specific locations given in the references allowed localisation with reasonable accuracy.

Table A.1: Organism metadata extracted from the database entries, or the respective literature, as available (see Table 2.2). The TEMP. column gives temperature ranges for organism growth, with the optimal growth temperature in grey. HABITAT reflects where the respective organism was isolated and does not necessarily represent its preferred life style.

\begin{tabular}{|c|c|c|c|c|}
\hline STRAIN & HABITAT & LOC. & TEMP. $\left[{ }^{\circ} \mathrm{C}\right]$ & ACCESSION \\
\hline $\begin{array}{l}\text { Aleiiroseovarius } \\
\text { crassostreae }\end{array}$ & $\begin{array}{l}\text { associated } \\
\text { host }\end{array}$ & n.a. & n.d. & $\begin{array}{l}\text { LKBA } \\
\text { oooooooo }\end{array}$ \\
\hline $\begin{array}{l}\text { Celeribacter } \\
\text { baekdonensis } \mathrm{B}_{30}\end{array}$ & sediment & n.a. & n.d. & $\begin{array}{l}\text { AMRK } \\
\text { OOOooooo }\end{array}$ \\
\hline Celeribacter indicus $\mathrm{P}_{73}$ & sediment & $\begin{array}{l}63.93 \mathrm{E} \\
27.85 \mathrm{~S}\end{array}$ & $10-41(28)$ & $\begin{array}{l}\text { GCA_ } \\
\text { ooo819565.1 }\end{array}$ \\
\hline Citreicella sp. 357 & sand & $\begin{array}{l}42.7748 \mathrm{~N} \\
9.1242 \mathrm{~W}\end{array}$ & n.d. & $\begin{array}{l}\text { AJKJ } \\
\text { ooooooooo }\end{array}$ \\
\hline Citreicella sp. SE45 & detritus & n.a. & n.d. & $\begin{array}{l}\text { ACNW } \\
\text { oooooooo }\end{array}$ \\
\hline $\begin{array}{l}\text { Rhodobacterales } \\
\text { bacterium HTCC } 2255\end{array}$ & planktonic & n.a. & n.d. & $\begin{array}{l}\text { NZ_AATR } \\
\text { oooooooo }\end{array}$ \\
\hline Phaeobacter sp. LSS9 & $\begin{array}{l}\text { associated } \\
\text { host }\end{array}$ & n.a. & n.d. & n.a. \\
\hline $\begin{array}{l}\text { Dinoroseobacter shibae } \\
\text { DSM } 16493\end{array}$ & $\begin{array}{l}\text { associated } \\
\text { patch }\end{array}$ & n.a. & $15-38(33)$ & $\begin{array}{l}\mathrm{NC}_{-} \\
\text {o09952 }\end{array}$ \\
\hline $\begin{array}{l}\text { Jannaschia aquimarina } \\
\text { GSW-M26 }\end{array}$ & planktonic & n.a. & $15-37(30)$ & $\begin{array}{l}\text { NZ_JYFE } \\
\text { oooooooo }\end{array}$ \\
\hline $\begin{array}{l}\text { Jannaschia rubra DSM } \\
16279\end{array}$ & planktonic & $\begin{array}{l}39.4423 \mathrm{~N} \\
0.284485 \mathrm{~W}\end{array}$ & $4-25$ & n.a. \\
\hline Jannaschia sp. CCS1 & planktonic & $\begin{array}{l}38.311 \mathrm{~N} \\
123.066 \mathrm{~W}\end{array}$ & n.d. & $\begin{array}{l}\text { GCA_ } \\
000013565.1\end{array}$ \\
\hline $\begin{array}{l}\text { Ketogulonicigenium } \\
\text { vulgare WSH-oo1 }\end{array}$ & n.a. & n.a. & n.d. & $\begin{array}{l}\mathrm{NC}_{-} \\
017384\end{array}$ \\
\hline $\begin{array}{l}\text { Ketogulonicigenium } \\
\text { vulgare } \mathrm{Y}_{25}\end{array}$ & terrestrial & n.a. & n.d. & $\begin{array}{l}\text { GCA }_{-} \\
000164885\end{array}$ \\
\hline $\begin{array}{l}\text { Loktanella atrilutea } \\
\text { DSM } 29326\end{array}$ & planktonic & n.a. & n.d. & $\begin{array}{l}\text { NZ_FQUE } \\
\text { oooooooo }\end{array}$ \\
\hline $\begin{array}{l}\text { Leisingera aquaemixtae } \\
\text { CECT } 8399\end{array}$ & planktonic & $\begin{array}{l}33.251944 \mathrm{~N} \\
126.623889 \mathrm{E}\end{array}$ & $10-40(30)$ & $\begin{array}{l}\text { NZ_CYSR } \\
\text { o0000000 }\end{array}$ \\
\hline $\begin{array}{l}\text { Leisingera aquimarina } \\
\text { DSM } 24565\end{array}$ & biofilm & $\begin{array}{l}44.4123 \mathrm{~N} \\
8.9264 \mathrm{E}\end{array}$ & $4-37(20)$ & $\begin{array}{l}\text { NZ_AXBE } \\
\text { oooooooo }\end{array}$ \\
\hline
\end{tabular}


Table A.1: continued

\begin{tabular}{|c|c|c|c|c|}
\hline STRAIN & HABITAT & LOC. & TEMP. $\left[{ }^{\circ} \mathrm{C}\right]$ & ACCESSION \\
\hline $\begin{array}{l}\text { Leisingera } \\
\text { methylohalidivorans } \\
\text { DSM } 14336\end{array}$ & planktonic & $\begin{array}{l}38.9 \mathrm{~N} \\
77.03 \mathrm{~W}\end{array}$ & $4-36(27)$ & $\begin{array}{l}\text { CP } \\
\text { o06773.1 }\end{array}$ \\
\hline $\begin{array}{l}\text { Sedimentalea } \\
\text { nanhaiensis DSM } 24252\end{array}$ & sediment & $\begin{array}{l}15.55 \mathrm{~N} ; \\
114.49 \mathrm{E}\end{array}$ & $4-37(25)$ & $\begin{array}{l}\text { NZ_AXBG } \\
\text { oooooooo }\end{array}$ \\
\hline $\begin{array}{l}\text { Loktanella fryxellensis } \\
\text { DSM } 16213\end{array}$ & $\begin{array}{l}\text { microbial } \\
\text { mat }\end{array}$ & $\begin{array}{l}77.6099 \mathrm{~S} \\
163.1555 \mathrm{E}\end{array}$ & $5^{-25}(25)$ & $\begin{array}{l}\text { NZ_FOCI } \\
\text { oooooooo }\end{array}$ \\
\hline $\begin{array}{l}\text { Litoreibacter albidus } \\
\text { DSM } 26922\end{array}$ & $\begin{array}{l}\text { associated } \\
\text { host }\end{array}$ & $\begin{array}{l}42.902 \mathrm{~N} \\
131.75 \mathrm{E}\end{array}$ & $4-37(26)$ & $\begin{array}{l}\text { NZ_FNOI } \\
\text { о0000000 }\end{array}$ \\
\hline $\begin{array}{l}\text { Litoreibacter arenae } \\
\text { DSM } 19593\end{array}$ & sand & $\begin{array}{l}36.048 \mathrm{~N} \\
129.584 \mathrm{E}\end{array}$ & $5-35(30)$ & $\begin{array}{l}\text { NZ_AONI } \\
\text { o0000000 }\end{array}$ \\
\hline $\begin{array}{l}\text { Litoreibacter } \\
\text { ascidiaceicola DSM } \\
100566\end{array}$ & $\begin{array}{l}\text { associated } \\
\text { host }\end{array}$ & $\begin{array}{l}37.801 \mathrm{~N} \\
129.021 \mathrm{E}\end{array}$ & $4-34(25)$ & $\begin{array}{l}\text { NZ_FQUV } \\
\text { oooooooo }\end{array}$ \\
\hline $\begin{array}{l}\text { Litoreibacter janthinus } \\
\text { DSM } 26921\end{array}$ & sediment & $\begin{array}{l}42.902 \mathrm{~N} \\
131.75 \mathrm{E}\end{array}$ & $4-37(26)$ & $\begin{array}{l}\text { NZ_FOYO } \\
\text { ooooooooo }\end{array}$ \\
\hline $\begin{array}{l}\text { Loktanella koreensis } \\
\text { DSM } 17925\end{array}$ & sand & $\begin{array}{l}36.048 \mathrm{~N} \\
129.584 \mathrm{E}\end{array}$ & $5-30$ & $\begin{array}{l}\text { NZ_FOIZ } \\
\text { oooooooo }\end{array}$ \\
\hline $\begin{array}{l}\text { Loktanella litorea DSM } \\
29433\end{array}$ & planktonic & n.a. & $15-37(30)$ & $\begin{array}{l}\text { NZ_FOZM } \\
\text { о0000000 }\end{array}$ \\
\hline $\begin{array}{l}\text { Loktanella cinnabarina } \\
\text { LL-o01 }\end{array}$ & sediment & $\begin{array}{l}41.17667 \mathrm{~N} \\
142.20056 \mathrm{E}\end{array}$ & $15-35(25)$ & $\begin{array}{l}\text { NZ_BATB } \\
\text { oooooooo }\end{array}$ \\
\hline $\begin{array}{l}\text { Loktanella } \\
\text { hongkongensis DSM } \\
17492\end{array}$ & biofilm & $\begin{array}{l}22.3 \mathrm{~N} \\
114.19 \mathrm{E}\end{array}$ & $8-44(30)$ & $\begin{array}{l}\text { NZ_APGJ } \\
\text { oooooooo }\end{array}$ \\
\hline Loktanella sp. SE62 & salt marsh & $\begin{array}{l}31.39 \mathrm{~N} \\
81.27 \mathrm{~W}\end{array}$ & n.d. & n.a. \\
\hline $\begin{array}{l}\text { Loktanella vestfoldensis } \\
\text { DSM } 16212\end{array}$ & $\begin{array}{l}\text { microbial } \\
\text { mat }\end{array}$ & $\begin{array}{l}\text { 68.4564S; } \\
78.1898 \mathrm{E}\end{array}$ & $5-37(20)$ & $\begin{array}{l}\text { NZ_ARNL } \\
\text { oоoooooo }\end{array}$ \\
\hline $\begin{array}{l}\text { Loktanella vestfoldensis } \\
\text { SKA53 }\end{array}$ & planktonic & $\begin{array}{l}58.94083 \mathrm{~N} \\
11.07944 \mathrm{E}\end{array}$ & n.d. & $\begin{array}{l}\text { NZ_AAMS } \\
\text { oooooooo }\end{array}$ \\
\hline $\begin{array}{l}\text { Loktanella pyoseonensis } \\
\text { DSM } 21424\end{array}$ & sand & $\begin{array}{l}33.328 \mathrm{~N} \\
126.842 \mathrm{E}\end{array}$ & $4-30(25)$ & $\begin{array}{l}\text { NZ_FNAT } \\
\text { oooooooo }\end{array}$ \\
\hline $\begin{array}{l}\text { Loktanella rosea DSM } \\
29591\end{array}$ & sediment & $\begin{array}{l}42.908 \mathrm{~N} \\
132.349 \mathrm{E}\end{array}$ & $4-35(25)$ & $\begin{array}{l}\text { NZ_FTPR } \\
\text { oooooooo }\end{array}$ \\
\hline $\begin{array}{l}\text { Loktanella salsilacus } \\
\text { DSM } 16199\end{array}$ & $\begin{array}{l}\text { microbial } \\
\text { mat }\end{array}$ & $\begin{array}{l}68.4593 \mathrm{~S} ; \\
78.1884 \mathrm{E}\end{array}$ & $5-30(25)$ & $\begin{array}{l}\text { NZ_FOTF } \\
\text { oooooooo }\end{array}$ \\
\hline $\begin{array}{l}\text { Loktanella sediminum } \\
\text { DSM } 28715\end{array}$ & sediment & $\begin{array}{l}30.243333 \mathrm{~N} \\
128.4475 \mathrm{E}\end{array}$ & $10-46(30)$ & $\begin{array}{l}\text { NZ_FQXB } \\
\text { oooooooo }\end{array}$ \\
\hline $\begin{array}{l}\text { Loktanella tamlensis } \\
\text { DSM } 26879\end{array}$ & planktonic & $\begin{array}{l}33.5277 \mathrm{~N} \\
126.5856 \mathrm{E}\end{array}$ & $4-30(27)$ & $\begin{array}{l}\text { NZ_FOYP } \\
\text { o0000000 }\end{array}$ \\
\hline $\begin{array}{l}\text { Marinovum algicola } \\
\text { DG } 898\end{array}$ & $\begin{array}{l}\text { associated } \\
\text { patch }\end{array}$ & n.a. & n.d. & $\begin{array}{l}\text { GCF }_{-} \\
001046955\end{array}$ \\
\hline
\end{tabular}


Table A.1: continued

\begin{tabular}{|c|c|c|c|c|}
\hline STRAIN & HABITAT & LOC. & TEMP. $\left[{ }^{\circ} \mathrm{C}\right]$ & ACCESSION \\
\hline $\begin{array}{l}\text { Maritimibacter } \\
\text { alkaliphilus HTCC } 2654\end{array}$ & n.a. & $\begin{array}{l}31.66667 \mathrm{~N} \\
64.16667 \mathrm{~W}\end{array}$ & $16-37(30)$ & $\begin{array}{l}\text { NZ_AAMT } \\
\text { o0000000 }\end{array}$ \\
\hline $\begin{array}{l}\text { Parvularcula } \\
\text { bermudensis HTCC } \\
2503\end{array}$ & planktonic & $\begin{array}{l}31.66667 \mathrm{~N} ; \\
64.16667 \mathrm{~W}\end{array}$ & $10-37(30)$ & $\begin{array}{l}\text { NZ_AAMU } \\
\text { 01000000 }\end{array}$ \\
\hline $\begin{array}{l}\text { Maritimibacter sp. } \\
\text { HL-12 }\end{array}$ & n.a. & n.a. & n.d. & $\begin{array}{l}\text { NZ_FXBQ } \\
\text { oooooooo }\end{array}$ \\
\hline $\begin{array}{l}\text { Nautella italica DSM } \\
26436\end{array}$ & biofilm & $\begin{array}{l}44.404 \mathrm{~N} \\
8.923 \mathrm{E}\end{array}$ & $4-45(25)$ & $\begin{array}{l}\text { GCA_ } \\
900113345\end{array}$ \\
\hline Nautella italica R11 & $\begin{array}{l}\text { associated } \\
\text { host }\end{array}$ & $\begin{array}{l}33.99389 \mathrm{~S} \\
151.26667 \mathrm{E}\end{array}$ & n.d. & $\begin{array}{l}\text { NZ_ABXM } \\
\text { o0000000 }\end{array}$ \\
\hline $\begin{array}{l}\text { Nereida ignava CECT } \\
5292\end{array}$ & planktonic & $\begin{array}{l}39.4778 \mathrm{~N} \\
0.281 \mathrm{~W}\end{array}$ & $10-30$ & $\begin{array}{l}\text { NZ_CVPC } \\
\text { oooooooo }\end{array}$ \\
\hline $\begin{array}{l}\text { Oceanibulbus indolifex } \\
\text { HEL-45 }\end{array}$ & planktonic & $\begin{array}{l}54.13333 \mathrm{~N} \\
7.86667 \mathrm{E}\end{array}$ & n.d. & $\begin{array}{l}\text { NZ_ABID } \\
\text { oooooooo }\end{array}$ \\
\hline $\begin{array}{l}\text { Oceanicola batsensis } \\
\text { HTCC } 2597\end{array}$ & planktonic & $\begin{array}{l}31.66667 \mathrm{~N} ; \\
64.16667 \mathrm{~W}\end{array}$ & $4-40(30)$ & $\begin{array}{l}\text { NZ_AAMO } \\
\text { o0000000 }\end{array}$ \\
\hline $\begin{array}{l}\text { Oceanicola granulosus } \\
\text { HTCC } 2516\end{array}$ & planktonic & $\begin{array}{l}31.66667 \mathrm{~N} \\
64.16667 \mathrm{~W}\end{array}$ & $4-40(28)$ & $\begin{array}{l}\text { NZ_AAOT } \\
\text { о0000000 }\end{array}$ \\
\hline $\begin{array}{l}\text { Oceanicola nanhaiensis } \\
\text { DSM } 18065\end{array}$ & sediment & n.a. & $10-37(28)$ & $\begin{array}{l}\text { NZ_JHZF } \\
\text { oooooooo }\end{array}$ \\
\hline Oceanicola sp. HL-35 & n.a. & n.a. & n.d. & $\begin{array}{l}\text { NZ_JAFT } \\
\text { oooooooo }\end{array}$ \\
\hline $\begin{array}{l}\text { Oceanicola sp. } \\
\text { MCTG1561a }\end{array}$ & n.a. & $\begin{array}{l}56.4397 \mathrm{~N} \\
5.5449 \mathrm{~W}\end{array}$ & n.d. & $\begin{array}{l}\text { NZ_JQMY } \\
\text { o0000000 }\end{array}$ \\
\hline Oceanicola sp. S124 & planktonic & $\begin{array}{l}7.4 \mathrm{~N} \\
151.75 \mathrm{E}\end{array}$ & n.d. & $\begin{array}{l}\text { GCA_ } \\
000220565.2\end{array}$ \\
\hline $\begin{array}{l}\text { Oceaniovalibus } \\
\text { guishaninsula JLT2003 }\end{array}$ & planktonic & $\begin{array}{l}24.845 \mathrm{~N} \\
121.94 \mathrm{E}\end{array}$ & $16-40(25)$ & $\begin{array}{l}\text { NZ_AMGO } \\
\text { oooooooo }\end{array}$ \\
\hline $\begin{array}{l}\text { Octadecabacter } \\
\text { antarcticus } 307\end{array}$ & ice & $\begin{array}{l}77.883333 \mathrm{~S} \\
166.583333 \mathrm{E}\end{array}$ & $4-10$ & $\begin{array}{l}\text { GCA_ } \\
\text { o00155735.2 }\end{array}$ \\
\hline $\begin{array}{l}\text { Octadecabacter arcticus } \\
\text { DSM } 13978\end{array}$ & ice & $\begin{array}{l}73.016667 \mathrm{~N} ; \\
148.516667 \mathrm{E}\end{array}$ & $4-15$ & $\begin{array}{l}\text { GCA_ } \\
000155675.2\end{array}$ \\
\hline $\begin{array}{l}\text { Octadecabacter } \\
\text { ascidiaceicola CECT } \\
8868\end{array}$ & $\begin{array}{l}\text { associated } \\
\text { host }\end{array}$ & $\begin{array}{l}34.836 \mathrm{~N} \\
128.447 \mathrm{E}\end{array}$ & $10-30(25)$ & $\begin{array}{l}\text { NZ_FXYD } \\
\text { o0000000 }\end{array}$ \\
\hline $\begin{array}{l}\text { Pseudooctadecabacter } \\
\text { jejudonensis CECT } 8397\end{array}$ & planktonic & $\begin{array}{l}33.2525 \mathrm{~N} \\
126.6236 \mathrm{E}\end{array}$ & $15-35(30)$ & n.a. \\
\hline Octadecabacter sp. E8 & $\begin{array}{l}\text { associated } \\
\text { host }\end{array}$ & $\begin{array}{l}53.703999 \mathrm{~N} ; \\
7.703611 \mathrm{E}\end{array}$ & n.d. & n.a. \\
\hline $\begin{array}{l}\text { Octadecabacter sp. } \\
\mathrm{NH}_{9}-\mathrm{P}_{7}\end{array}$ & sediment & $\begin{array}{l}53 \cdot 70466 \mathrm{~N} \\
7 \cdot 70447 \mathrm{E}\end{array}$ & n.d. & n.a. \\
\hline $\begin{array}{l}\text { Octadecabacter } \\
\text { temperatus Sb1 }\end{array}$ & planktonic & $\begin{array}{l}54.18 \mathrm{~N} \\
7.89 \mathrm{E}\end{array}$ & $4-25(20)$ & $\begin{array}{l}\text { GCA_ }_{-} \\
001187845\end{array}$ \\
\hline
\end{tabular}


Table A.1: continued

\begin{tabular}{|c|c|c|c|c|}
\hline STRAIN & HABITAT & LOC. & TEMP. $\left[{ }^{\circ} \mathrm{C}\right]$ & ACCESSION \\
\hline $\begin{array}{l}\text { Octadecabacter sp. } \\
\text { PAMC27224 }\end{array}$ & sediment & $\begin{array}{l}\text { 74.64611S; } \\
164.22333 \mathrm{E}\end{array}$ & $10-25$ & n.a. \\
\hline $\begin{array}{l}\text { Octadecabacter sp. } \\
\text { PAMC } 27225\end{array}$ & sediment & $\begin{array}{l}\text { 74.64611S; } \\
164.22333 \mathrm{E}\end{array}$ & $10-25$ & n.a. \\
\hline $\begin{array}{l}\text { Pelagibaca bermudensis } \\
\text { HTCC } 2601\end{array}$ & planktonic & $\begin{array}{l}31.66667 \mathrm{~N} \\
64.16667 \mathrm{~W}\end{array}$ & $10-40(30)$ & $\begin{array}{l}\text { NZ_AATQ } \\
\text { oooooooo }\end{array}$ \\
\hline $\begin{array}{l}\text { Pseudophaeobacter } \\
\text { arcticus DSM } 23566\end{array}$ & sediment & $\begin{array}{l}75.006667 \mathrm{~N} \\
169.993611 \mathrm{~W}\end{array}$ & $0-25(20)$ & $\begin{array}{l}\text { NZ_AXBF } \\
\text { oooooooo }\end{array}$ \\
\hline $\begin{array}{l}\text { Leisingera caerulea } \\
\text { DSM } 24564\end{array}$ & biofilm & $\begin{array}{l}44.404 \mathrm{~N} \\
8.923 \mathrm{E}\end{array}$ & $4-45(25)$ & $\begin{array}{l}\text { NZ_AXBI } \\
\text { oooooooo }\end{array}$ \\
\hline $\begin{array}{l}\text { Leisingera daeponensis } \\
\text { DSM } 23529\end{array}$ & sediment & $\begin{array}{l}33.2371 \mathrm{~N} \\
126.4248 \mathrm{E}\end{array}$ & $4-42$ & $\begin{array}{l}\text { NZ_AXBD } \\
\text { oooooooo }\end{array}$ \\
\hline $\begin{array}{l}\text { Phaeobacter gallaeciensis } \\
\text { ANG1 }\end{array}$ & $\begin{array}{l}\text { associated } \\
\text { host }\end{array}$ & $\begin{array}{l}21.269972 \mathrm{~N} \\
157.73739 \mathrm{~W}\end{array}$ & n.d. & $\begin{array}{l}\text { NZ_AFCF } \\
\text { 00000000 }\end{array}$ \\
\hline $\begin{array}{l}\text { Phaeobacter gallaeciensis } \\
\text { BS107 }\end{array}$ & planktonic & $\begin{array}{l}43.391910 \mathrm{~N} \\
8.403664 \mathrm{~W}\end{array}$ & $15-37(25)$ & $\begin{array}{l}\text { GCA_ } \\
000511385\end{array}$ \\
\hline $\begin{array}{l}\text { Phaeobacter gallaeciensis } \\
\text { DSM } 17395\end{array}$ & n.a. & n.a. & n.d. & $\begin{array}{l}\text { GCA_ } \\
\text { ooo154765 }\end{array}$ \\
\hline $\begin{array}{l}\text { Phaeobacter gallaeciensis } \\
\text { DSM } 26640\end{array}$ & planktonic & $\begin{array}{l}43.391910 \mathrm{~N} \\
8.403664 \mathrm{~W}\end{array}$ & $15-37(25)$ & $\begin{array}{l}\text { GCA_ } \\
000511385\end{array}$ \\
\hline $\begin{array}{l}\text { Phaeobacter inhibens } \\
\text { DSM } 16374\end{array}$ & planktonic & $\begin{array}{l}53.705556 \mathrm{~N} \\
7 \cdot 718722 \mathrm{E}\end{array}$ & $4-36(28)$ & $\begin{array}{l}\text { GCA_ } \\
000473105\end{array}$ \\
\hline $\begin{array}{l}\text { Planktomarina } \\
\text { temperata RCA23 }\end{array}$ & planktonic & $53.7 \mathrm{~N} ; 7.71 \mathrm{E}$ & $10-30(25)$ & $\begin{array}{l}\text { GCA_ }_{-} \\
000738435\end{array}$ \\
\hline $\begin{array}{l}\text { Rhodobacteraceae } \\
\text { bacterium } \mathrm{SB}_{2}\end{array}$ & planktonic & $\begin{array}{l}54.171943 \mathrm{~N} \\
7.894196 \mathrm{E}\end{array}$ & n.d. & $\begin{array}{l}\text { NZ_LGRT } \\
\text { oooooooo }\end{array}$ \\
\hline $\begin{array}{l}\text { Rhodobacterales sp. } \\
\text { HTCC } 2083\end{array}$ & planktonic & $\begin{array}{l}44.652137 \mathrm{~N} \\
124.070921 \mathrm{~W}\end{array}$ & n.d. & $\begin{array}{l}\text { NZ_ABXE } \\
\text { Oо0о0000 }\end{array}$ \\
\hline $\begin{array}{l}\text { Rhodobacterales sp. } \\
\text { HTCC } 2150\end{array}$ & planktonic & $\begin{array}{l}44.651667 \mathrm{~N} \\
124.411667 \mathrm{~W}\end{array}$ & n.d. & $\begin{array}{l}\text { NZ_AAZX } \\
\text { оо0о0000 }\end{array}$ \\
\hline Rhodobacterales sp. Y4I & planktonic & $\begin{array}{l}30.7236 \mathrm{~N} \\
81.5111 \mathrm{~W}\end{array}$ & n.d. & $\begin{array}{l}\text { NZ_ABXF } \\
\text { oоoooooo }\end{array}$ \\
\hline $\begin{array}{l}\text { Roseobacter denitrificans } \\
\text { OCh } 114\end{array}$ & $\begin{array}{l}\text { associated } \\
\text { host }\end{array}$ & $\begin{array}{l}35.159315 \mathrm{~N} \\
139.616576 \mathrm{E}\end{array}$ & $2-30$ & $\begin{array}{l}\text { GCA_ } \\
000014045 \cdot 1\end{array}$ \\
\hline $\begin{array}{l}\text { Roseobacter litoralis } \\
\text { Och } 149\end{array}$ & $\begin{array}{l}\text { associated } \\
\text { host }\end{array}$ & n.a. & $2-30$ & $\begin{array}{l}\text { GCA_ } \\
\text { o00154785.2 }\end{array}$ \\
\hline $\begin{array}{l}\text { Roseobacter sp. } \\
\text { AzwK-3b }\end{array}$ & planktonic & $\begin{array}{l}36.84262 \mathrm{~N} \\
121.74701 \mathrm{~W}\end{array}$ & n.d. & $\begin{array}{l}\text { GCA_ } \\
\text { ooo170875 }\end{array}$ \\
\hline Roseobacter sp. CCS2 & planktonic & $\begin{array}{l}38.308333 \mathrm{~N} \\
123.3 \mathrm{~W}\end{array}$ & n.d. & $\begin{array}{l}\text { NZ_AAYB } \\
\text { o0000000 }\end{array}$ \\
\hline Roseobacter sp. GAI101 & planktonic & n.a. & n.d. & $\begin{array}{l}\text { NZ_ABXS } \\
\text { oooooooo }\end{array}$ \\
\hline
\end{tabular}


Table A.1: continued

\begin{tabular}{|c|c|c|c|c|}
\hline STRAIN & HАВITAT & LOC. & TEMP. $\left[{ }^{\circ} \mathrm{C}\right]$ & ACCESSION \\
\hline Roseobacter sp. LE17 & n.a. & $\begin{array}{l}32.84 \mathrm{~N} ; \\
117.2769 \mathrm{~W}\end{array}$ & n.d. & n.a. \\
\hline $\begin{array}{l}\text { Roseobacter sp. } \\
\text { MED193 }\end{array}$ & planktonic & $\begin{array}{l}41.66667 \mathrm{~N} \\
2.8 \mathrm{E}\end{array}$ & n.d. & $\begin{array}{l}\text { NZ_AANB } \\
\text { oooooooo }\end{array}$ \\
\hline Roseobacter sp. R2A57 & planktonic & $\begin{array}{l}44.653167 \mathrm{~N} \\
124.178167 \mathrm{~W}\end{array}$ & n.d. & n.a. \\
\hline $\begin{array}{l}\text { Roseobacter sp. } \\
\text { SK209-2-6 }\end{array}$ & n.a. & n.a. & n.d. & $\begin{array}{l}\text { NZ_AAYC } \\
\text { oooooooo }\end{array}$ \\
\hline $\begin{array}{l}\text { Roseovarius mucosus } \\
\text { DSM } 17069\end{array}$ & $\begin{array}{l}\text { associated } \\
\text { patch }\end{array}$ & $\begin{array}{l}54.195387 \mathrm{~N} \\
7.893448 \mathrm{E}\end{array}$ & $15-43(31)$ & $\begin{array}{l}\text { NZ_AONH } \\
\text { oooooooo }\end{array}$ \\
\hline $\begin{array}{l}\text { Roseovarius } \\
\text { nubinhibens ISM }\end{array}$ & planktonic & $\begin{array}{l}22.066667 \mathrm{~N} ; \\
74.066667 \mathrm{~W}\end{array}$ & $10-40(30)$ & $\begin{array}{l}\text { NZ_AALY } \\
\text { o0000000 }\end{array}$ \\
\hline $\begin{array}{l}\text { Roseovarius sp. } \\
\text { MCTG1562b }\end{array}$ & $\begin{array}{l}\text { associated } \\
\text { patch }\end{array}$ & $\begin{array}{l}56.332304 \mathrm{~N} \\
5 \cdot 748674 \mathrm{~W}\end{array}$ & n.d. & $\begin{array}{l}\text { NZ_JQLS } \\
\text { oooooooo }\end{array}$ \\
\hline $\begin{array}{l}\text { Roseovarius sp. } \\
\text { TM1035 }\end{array}$ & $\begin{array}{l}\text { associated } \\
\text { patch }\end{array}$ & n.a. & n.d. & $\begin{array}{l}\text { NZ_ABCL } \\
\text { oooooooo }\end{array}$ \\
\hline $\begin{array}{l}\text { Ruegeria atlantica DSM } \\
5823\end{array}$ & sediment & $\begin{array}{l}21.41 \mathrm{~N} ; \\
17.893333 \mathrm{~W}\end{array}$ & n.d. & $\begin{array}{l}\text { NZ_CYPU } \\
\text { oooooooo }\end{array}$ \\
\hline $\begin{array}{l}\text { Ruegeria halocynthiae } \\
\text { MOLA R1 13b }\end{array}$ & $\begin{array}{l}\text { associated } \\
\text { host }\end{array}$ & $\begin{array}{l}48.695278 \mathrm{~N} \\
3.143889 \mathrm{E}\end{array}$ & n.d. & $\begin{array}{l}\text { NZ_JQEZ } \\
\text { oooooooo }\end{array}$ \\
\hline $\begin{array}{l}\text { Ruegeria } \\
\text { lacuscaerulensis } \\
\text { ITI-1157 }\end{array}$ & n.a. & $\begin{array}{l}63.879 \mathrm{~N} \\
22.434 \mathrm{~W}\end{array}$ & n.d. & $\begin{array}{l}\text { NZ_ACNX } \\
\text { oooooooo }\end{array}$ \\
\hline Ruegeria mobilis F1926 & planktonic & $\begin{array}{l}31.4061 S ; \\
91.17758 \mathrm{E}\end{array}$ & n.d. & $\begin{array}{l}\text { NZ_CP } \\
015230.1\end{array}$ \\
\hline $\begin{array}{l}\text { Ruegeria pomeroyi } \\
\text { DSS-3 }\end{array}$ & planktonic & n.a. & $10-40$ & $\begin{array}{l}\text { GCA } \\
000011965.2\end{array}$ \\
\hline Ruegeria sp. TM1040 & $\begin{array}{l}\text { associated } \\
\text { patch }\end{array}$ & n.a. & n.d. & $\begin{array}{l}\text { NZ_AAFG } \\
\text { o0000000 }\end{array}$ \\
\hline Ruegeria sp. TW15 & $\begin{array}{l}\text { associated } \\
\text { host }\end{array}$ & n.a. & $10-37(27)$ & $\begin{array}{l}\text { NZ_AEYW } \\
\text { oooooooo }\end{array}$ \\
\hline Sagittula stellata E-37 & planktonic & n.a. & $10-41(30)$ & $\begin{array}{l}\text { NZ_AAYA } \\
\text { oooooooo }\end{array}$ \\
\hline $\begin{array}{l}\text { Shimia marina CECT } \\
7688\end{array}$ & biofilm & $\begin{array}{l}34.772850 \mathrm{ON} \\
128.387213 \mathrm{E}\end{array}$ & $15-35(33)$ & $\begin{array}{l}\text { NZ_CYPW } \\
\text { oooooooo }\end{array}$ \\
\hline Shimia sp. SKo13 & sediment & $\begin{array}{l}57.6125 \mathrm{~N} \\
8.59 \mathrm{E}\end{array}$ & $10-35(30)$ & $\begin{array}{l}\text { NZ_LAJH } \\
\text { oooooooo }\end{array}$ \\
\hline $\begin{array}{l}\text { Sulfitobacter donghicola } \\
\text { KCTC } 12864\end{array}$ & planktonic & $\begin{array}{l}37.240459 \mathrm{~N} \\
131.866944 \mathrm{E}\end{array}$ & $10-31(25)$ & $\begin{array}{l}\text { NZ_JASF } \\
\text { oooooooo }\end{array}$ \\
\hline $\begin{array}{l}\text { Sulfitobacter donghicola } \\
\text { JCM } 14565\end{array}$ & planktonic & $\begin{array}{l}37.240459 \mathrm{~N} \\
131.866944 \mathrm{E}\end{array}$ & $10-31(25)$ & $\begin{array}{l}\text { NZ_JASF } \\
\text { oooooooo }\end{array}$ \\
\hline $\begin{array}{l}\text { Sulfitobacter geojensis } \\
\text { MM-124 }\end{array}$ & planktonic & $\begin{array}{l}35.0575 \mathrm{~N} \\
128.803056 \mathrm{E}\end{array}$ & $4-30(25)$ & $\begin{array}{l}\text { NZ_JASE } \\
\text { oooooooo }\end{array}$ \\
\hline
\end{tabular}


Table A.1: continued

\begin{tabular}{|c|c|c|c|c|}
\hline STRAIN & HABITAT & LOC. & TEMP. $\left[{ }^{\circ} \mathrm{C}\right]$ & ACCESSION \\
\hline $\begin{array}{l}\text { Sulfitobacter guttiformis } \\
\text { KCTC } 32187\end{array}$ & planktonic & $\begin{array}{l}68.521 S \\
78.270 \mathrm{E}\end{array}$ & $4-32(16)$ & $\begin{array}{l}\text { NZ_JASG } \\
\text { oooooooo }\end{array}$ \\
\hline $\begin{array}{l}\text { Sulfitobacter } \\
\text { mediterraneus KCTC } \\
32188\end{array}$ & planktonic & $\begin{array}{l}42.516667 \mathrm{~N} \\
3.183333 \mathrm{E}\end{array}$ & $4-35(22)$ & $\begin{array}{l}\text { NZ_JASH } \\
\text { oooooooo }\end{array}$ \\
\hline $\begin{array}{l}\text { Sulfitobacter pontiacus } \\
\text { 3SOLIMARo9 }\end{array}$ & planktonic & $\begin{array}{l}39.794141 \mathrm{~N} \\
2.693348 \mathrm{E}\end{array}$ & n.d. & $\begin{array}{l}\text { NZ_AXZR } \\
\text { oooooooo }\end{array}$ \\
\hline $\begin{array}{l}\text { Sulfitobacter sp. } 20 \\
\text { GPM-1509m }\end{array}$ & n.a. & n.a. & n.d. & $\begin{array}{l}\text { NZ_JIBC } \\
\text { oooooooo }\end{array}$ \\
\hline $\begin{array}{l}\text { Sulfitobacter sp. } \\
\text { CB2047 }\end{array}$ & n.a. & n.a. & n.d. & $\begin{array}{l}\text { NZ_JPOY } \\
\text { oooooooo }\end{array}$ \\
\hline Sulfitobacter sp EE-36 & planktonic & n.a. & n.d. & $\begin{array}{l}\text { NZ_AALV } \\
\text { oooooooo }\end{array}$ \\
\hline $\begin{array}{l}\text { Sulfitobacter sp. MCCC } \\
1 \text { Aoo686 }\end{array}$ & $\begin{array}{l}\text { associated } \\
\text { patch }\end{array}$ & n.a. & $10-37(28)$ & $\begin{array}{l}\text { NZ_JAMD } \\
\text { oоoooooo }\end{array}$ \\
\hline $\begin{array}{l}\text { Sulfitobacter sp. } \\
\text { NAS-14.1 }\end{array}$ & planktonic & $\begin{array}{l}33 \cdot 3 \mathrm{~N} \\
74 \cdot 3 \mathrm{~W}\end{array}$ & n.d. & $\begin{array}{l}\text { NZ_AALZ } \\
\text { о0000000 }\end{array}$ \\
\hline Sulfitobacter sp. NB-68 & n.a. & $\begin{array}{l}35.658333 \mathrm{~N} \\
129.1275 \mathrm{E}\end{array}$ & $4-30(30)$ & $\begin{array}{l}\text { NZ_JASC } \\
\text { oooooooo }\end{array}$ \\
\hline Sulfitobacter sp. NB-77 & n.a. & $\begin{array}{l}35.658333 \mathrm{~N} \\
129.1275 \mathrm{E}\end{array}$ & $4-30(25)$ & $\begin{array}{l}\text { NZ_JASD } \\
\text { oоoooooo }\end{array}$ \\
\hline Sulfitobacter sp. SA11 & $\begin{array}{l}\text { associated } \\
\text { patch }\end{array}$ & $\begin{array}{l}47 \cdot 727888 \mathrm{~N} \\
122.473801 \mathrm{~W}\end{array}$ & n.d. & n.a. \\
\hline $\begin{array}{l}\text { Tateyamaria sp. ANG } \\
\text { S1 }\end{array}$ & $\begin{array}{l}\text { associated } \\
\text { host }\end{array}$ & $\begin{array}{l}21.269972 \mathrm{~N} \\
157 \cdot 73739 \mathrm{~W}\end{array}$ & n.d. & $\begin{array}{l}\text { NZ_JWLL } \\
\text { oooooooo }\end{array}$ \\
\hline $\begin{array}{l}\text { Thalassiobium sp. } \\
\text { R2A62 }\end{array}$ & planktonic & $\begin{array}{l}44.65 \mathrm{~N} ; \\
124.167 \mathrm{~W}\end{array}$ & n.d. & $\begin{array}{l}\text { NZ_ACOA } \\
\text { oooooooo }\end{array}$ \\
\hline $\begin{array}{l}\text { Thalassobacter } \\
\text { stenotrophicus CECT } \\
5294\end{array}$ & n.a. & n.a. & $13-37(24)$ & $\begin{array}{l}\text { NZ_CYRX } \\
\text { o0000000 }\end{array}$ \\
\hline $\begin{array}{l}\text { Wenxinia marina DSM } \\
24838\end{array}$ & sediment & $\begin{array}{l}22.83021 \mathrm{~N} \\
118.088829 \mathrm{E}\end{array}$ & $15-42(36)$ & $\begin{array}{l}\text { NZ_ARAY } \\
\text { oooooooo }\end{array}$ \\
\hline $\begin{array}{l}\text { Roseovarius atlanticus } \\
\text { R12B }\end{array}$ & planktonic & $15.2 S ; 13.3 \mathrm{~W}$ & $4-45(27)$ & $\begin{array}{l}\text { NZ_LAXJ } \\
\text { oоoooooo }\end{array}$ \\
\hline Roseovarius sp. 217 & planktonic & $\begin{array}{l}50.25 \mathrm{~N} \\
4.21667 \mathrm{~W}\end{array}$ & n.d. & $\begin{array}{l}\text { NZ_AAMV } \\
\text { oooooooo }\end{array}$ \\
\hline
\end{tabular}




\section{A.3 ROSEOBACTER GROUP PHYLOGENY AND OTHER TREES}

The full super-matrix-based phylogeny of 115 roseobacters plus outgroup organism is given in Figure A.2 (see Section 2.6 for method).

Gene-content-based hierarchical clustering (Figure 4.3) was repeated, deleting the core-genes of 22 sub-clades within the phylogeny in all members of that clade prior to computing Jaccard distances (Figure A.3).

In addition, strains were clustered based on the distances (in kilometres) between their isolation coordinates listed in Table A.1 (Figure A.4).

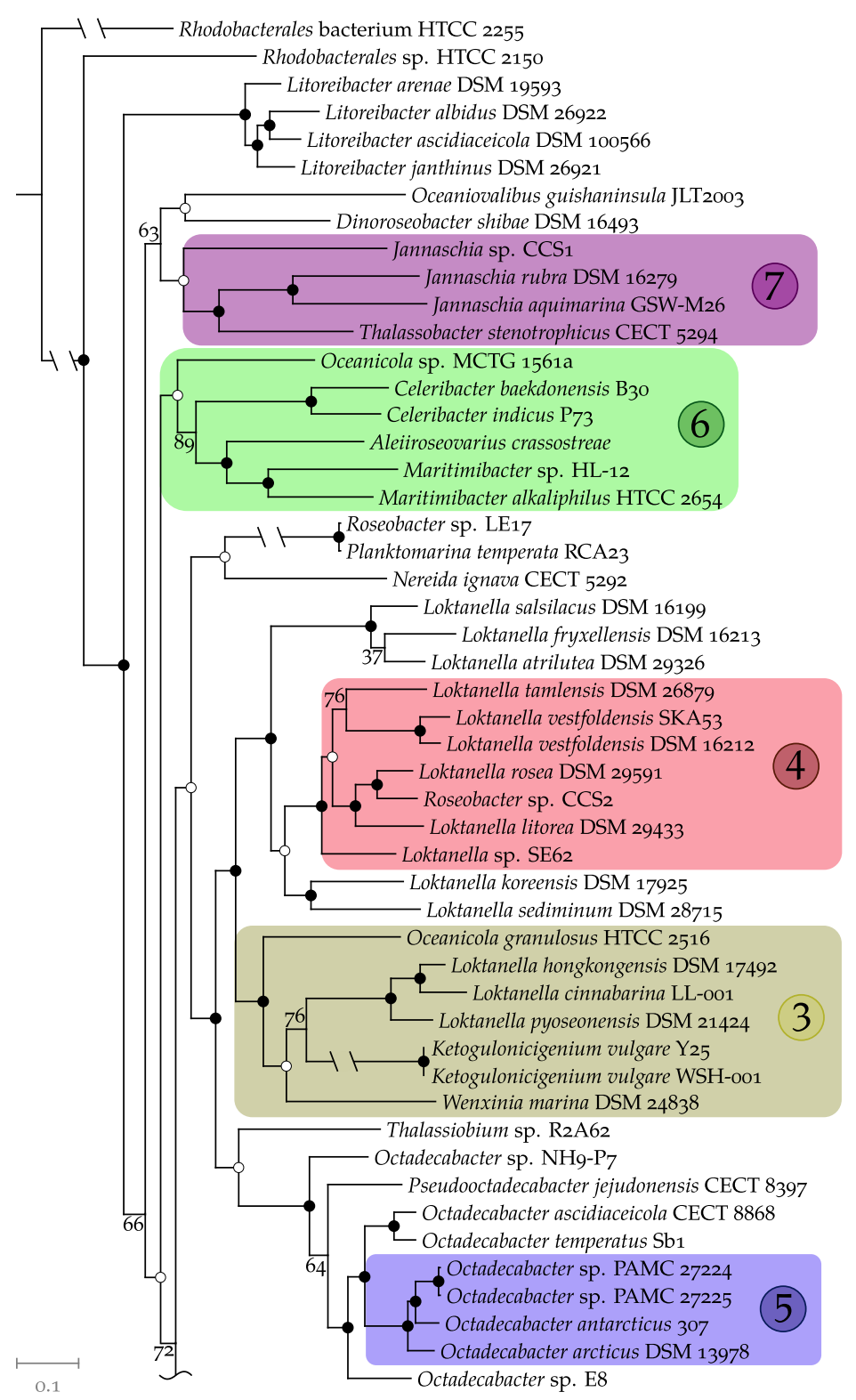

Figure A.2: Super-matrix-based core-genome phylogeny of 115 Roseobacter group genomes. Highlighted clades as defined in ref. [59]. Numbers indicate bootstrap support. Open and closed circles represent values of 90-99 and 100, respectively. Scale bar in expected number of substitutions. Long edges were shortened by $50 \%$ (marked by interruptions). Tree continued on next page. 


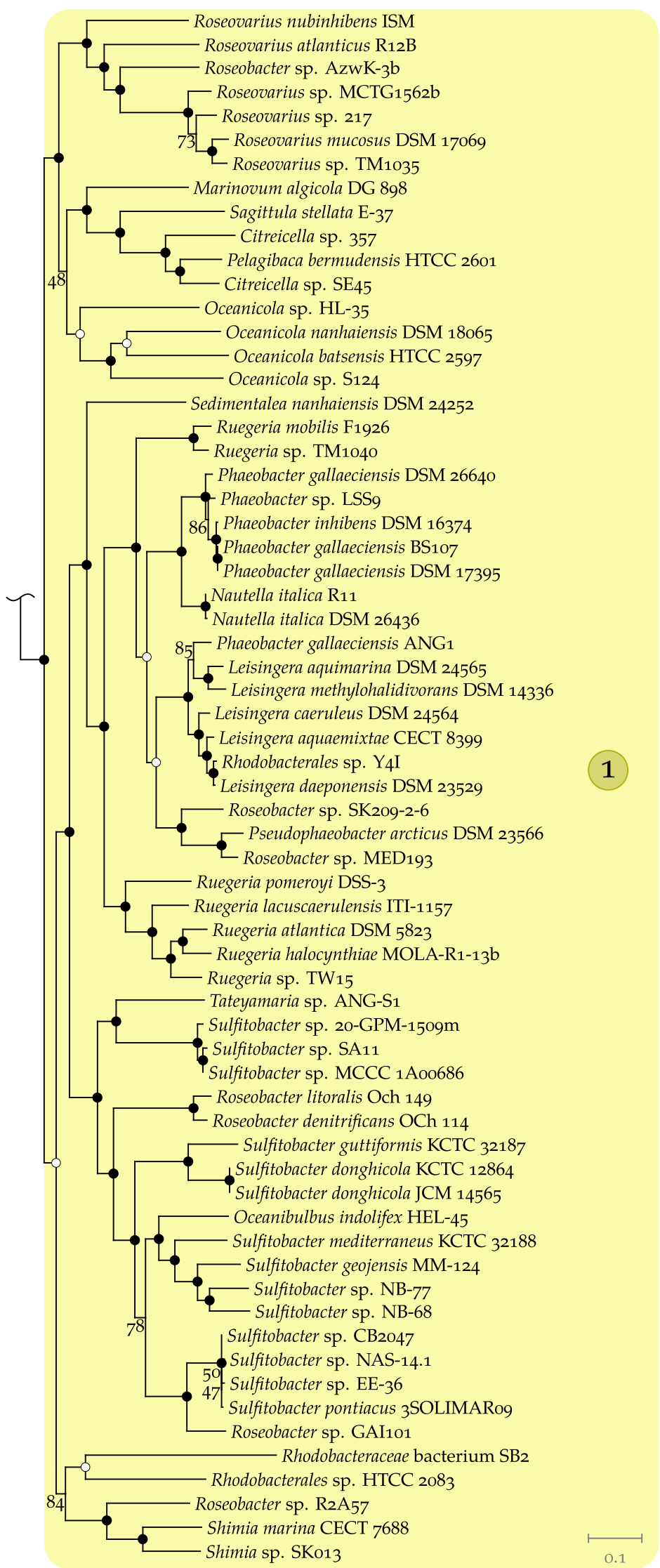

Figure A.2: Roseobacter group MLSA phylogeny (continued) 
CONTENT-BASED CLUSTERING

(excl. sub-clade core-genes)

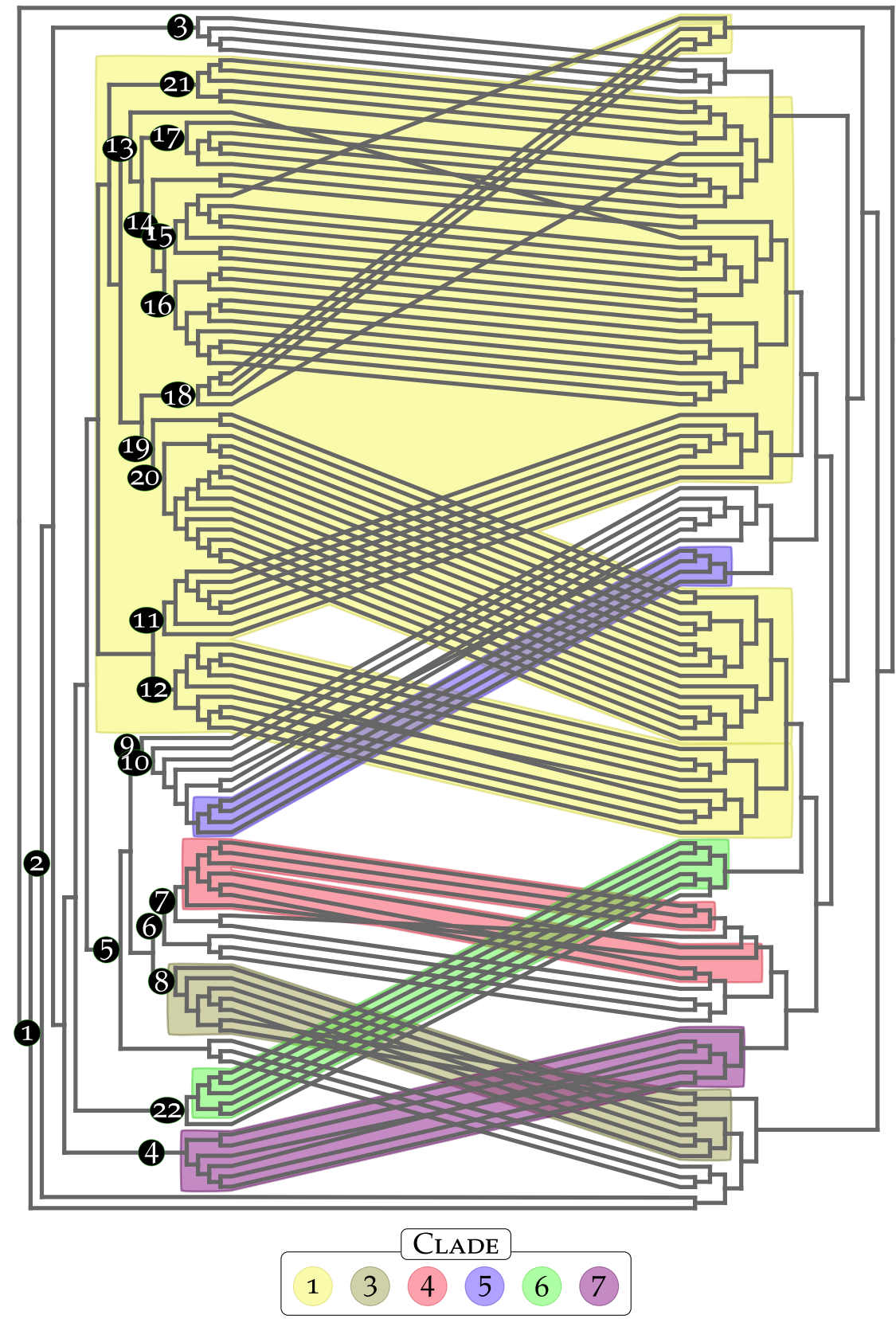

Figure A.3: MLSA phylogeny of 115 roseobacter genomes plus outgroup on the left, as given in Figure A.2, versus hierarchical clustering of the same genomes on the right, based on pairwise Jaccard distances of OG presence and absence. Before clustering, core-genes of sub-clades (labelled 1-22 in the figure) were removed from all members of that clade (that is, they were marked as absent prior to the Jaccard distance calculation) in numerical order. Clades defined in ref. [59] are coloured as in Figure A.2. RF distance between both trees is 0.47, determined using ete3 [177]. 


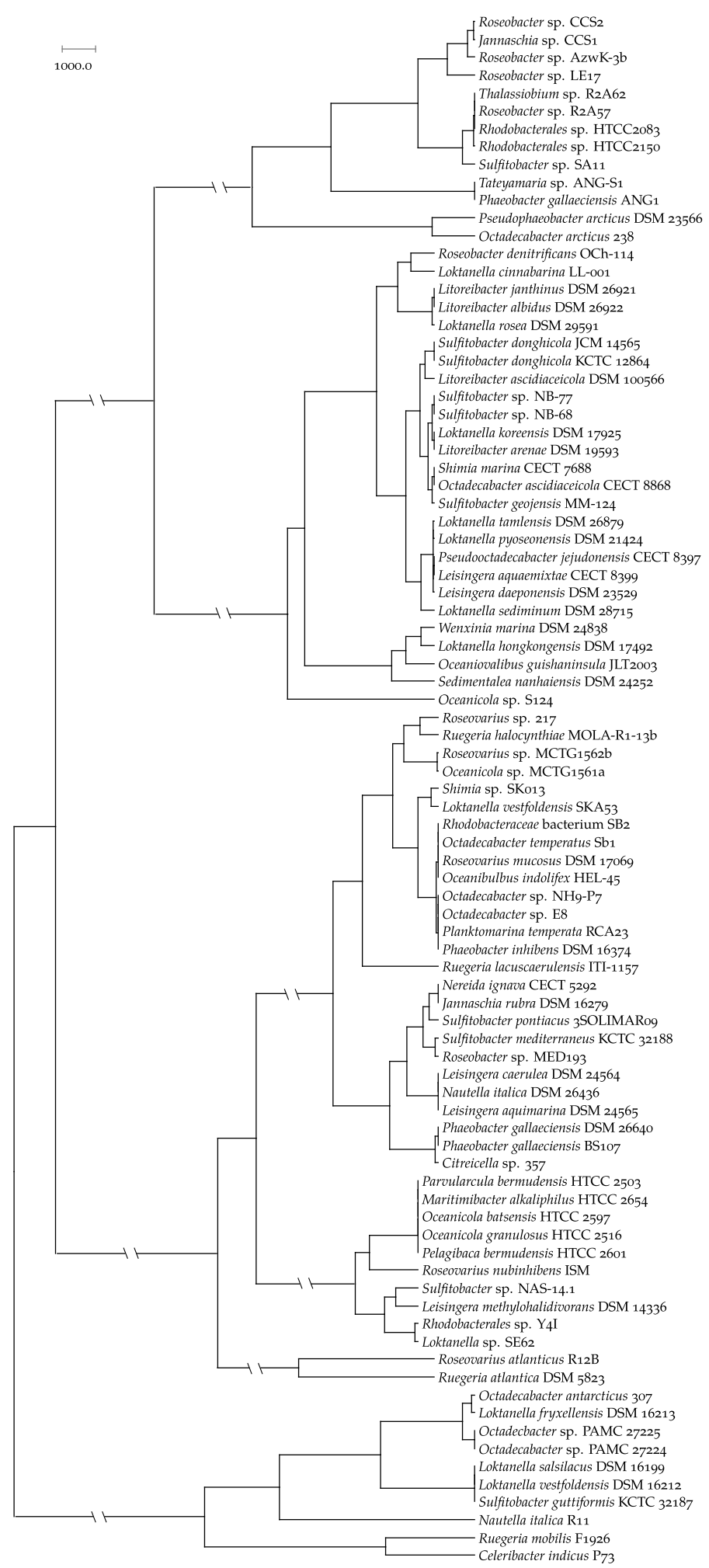

Figure A.4: Hierarchical clustering of genomes based on geographic distance (in kilometres). Only strains with available isolation coordinates (Table A.1) were used in the computation. RF distance to MLSA phylogeny (Figure A.2), gene content clustering, and cloud- and shell-genome clustering (right trees in Figures 4.3 and A.3, respectively) is 0.96, determined using ete3 [177]. Interrupted edges were shortened by $50 \%$. 


\section{A.4 GROUP-SPECIFIC GENES}

Genes specific to each group of Octadecabacter genomes, as defined in Section 2.10.2, are listed in Tables A.2 to A.5 for each group individually.

Table A.2: Genes exclusive to the group formed by O. arcticus, O. antarcticus, and isolates PAMC 27224 and 27225 (group I, Section 3.2), as determined by po2group_stats.pl. For each specific OG, the annotation and LOCUS TAG in the genome of isolate PAMC 27224 is provided for reference.

\begin{tabular}{|c|c|}
\hline DESCRIPTION & LOCUS TAG \\
\hline Site-specific DNA recombinase & octa_ooogo \\
\hline Protein of unknown function (DUF3987) & octa_o0390 \\
\hline hypothetical protein & octa_02100 \\
\hline hypothetical protein & octa_02490 \\
\hline CBS domain-containing protein & octa_02830 \\
\hline Protein of unknown function (DUF2848) & octa_03810 \\
\hline Uncharacterized conserved protein & octa_04100 \\
\hline RNA polymerase $\sigma^{32}$ factor & octa_06070 \\
\hline $\begin{array}{r}\text { Fe-S cluster biogenesis protein } \mathrm{NfuA}, 4 \mathrm{Fe}-4 \mathrm{~S} \text {-binding } \\
\text { domain }\end{array}$ & octa_06o8o \\
\hline $\begin{array}{r}\text { ATP-binding protein involved in chromosome } \\
\text { partitioning }\end{array}$ & octa_0609o \\
\hline DNA-binding transcriptional regulator, MarR family & octa_06100 \\
\hline Glycine/D-amino acid oxidase (deaminating) & octa_06740 \\
\hline transcriptional regulator, TetR family & octa_09560 \\
\hline aldehyde dehydrogenase $\left(\mathrm{NAD}^{+}\right)$ & octa_og610 \\
\hline cysteine desulfurase family protein, VC1184 subfamily & octa_09620 \\
\hline bile acid: $\mathrm{Na}^{+}$symporter, BASS family & octa_10390 \\
\hline AAA ATPase domain-containing protein & octa_10590 \\
\hline hypothetical protein (DUF2333) & octa_106oo \\
\hline hypothetical protein & octa_10610 \\
\hline 4- $\alpha$-glucanotransferase & octa_10680 \\
\hline Taurine catabolism dioxygenase TauD, TfdA family & octa_12020 \\
\hline SSU ribosomal protein $\mathrm{S}_{21} \mathrm{P}$ & octa_12240 \\
\hline TIGRo2453 family protein & octa_12280 \\
\hline monovalent cation: $\mathrm{H}^{+}$antiporter, CPA1 family & octa_12300 \\
\hline transposase & octa_13300 \\
\hline $\begin{array}{l}\text { Uncharacterized membrane protein } \mathrm{YdjX}, \mathrm{TVP}_{3} 8 / \\
\text { TMEM64 family, SNARE-associated domain }\end{array}$ & octa_13530 \\
\hline selenium-binding protein 1 & octa_13670 \\
\hline hypothetical protein & octa_13680 \\
\hline hypothetical protein & octa_14030 \\
\hline$\beta$-carotene $15,15^{\prime}$-monooxygenase, Brp/Blh family & octa_14420 \\
\hline
\end{tabular}


Table A.2: continued

\begin{tabular}{|c|c|}
\hline DESCRIPTION & LOCUS TAG \\
\hline lycopene beta-cyclase & octa_14430 \\
\hline phytoene synthase & octa_14440 \\
\hline geranylgeranyl diphosphate synthase, type II & octa_14460 \\
\hline Bacteriorhodopsin & octa_14470 \\
\hline $\begin{array}{r}\text { Predicted thiol-disulfide oxidoreductase YuxK, DCC } \\
\text { family }\end{array}$ & octa_14550 \\
\hline hypothetical protein & octa_16050 \\
\hline $\begin{array}{r}\text { ATP-binding protein involved in chromosome } \\
\text { partitioning }\end{array}$ & octa_16490 \\
\hline $\begin{array}{r}\mathrm{Fe}-\mathrm{S} \text { cluster biogenesis protein } \mathrm{NfuA}, 4 \mathrm{Fe}-4 \mathrm{~S}-\text { binding } \\
\text { domain }\end{array}$ & octa_16500 \\
\hline Acetyltransferase (GNAT) domain-containing protein & octa_19650 \\
\hline $\begin{array}{r}\text { Hemolysin-type calcium-binding repeat-containing } \\
\text { protein }\end{array}$ & octa_20610 \\
\hline PilZ domain-containing protein & octa_23420 \\
\hline $\begin{array}{r}\text { myo-inositol 2-dehydrogenase / D-chiro-inositol } \\
\text { 1-dehydrogenase }\end{array}$ & octa_25790 \\
\hline 3-hydroxyisobutyrate dehydrogenase & octa_25810 \\
\hline pyruvate dehydrogenase E1 component alpha subunit & octa_25900 \\
\hline pyruvate dehydrogenase E1 component beta subunit & octa_25910 \\
\hline $\begin{array}{r}\text { 2-oxoglutarate dehydrogenase E2 component } \\
\text { (dihydrolipoamide succinyltransferase) }\end{array}$ & octa_25920 \\
\hline $\begin{array}{l}\text { succinate-semialdehyde dehydrogenase / } \\
\text { glutarate-semialdehyde dehydrogenase }\end{array}$ & octa_25930 \\
\hline hypothetical protein & octa_26240 \\
\hline hypothetical protein & octa_27350 \\
\hline DNA polymerase $\mathrm{V}$ & octa_27360 \\
\hline simple sugar transport system ATP-binding protein & octa_28990 \\
\hline Alcohol dehydrogenase, class IV & octa_29950 \\
\hline Acyl-CoA reductase & octa_29960 \\
\hline glutamine synthetase & octa_29970 \\
\hline Predicted N-formylglutamate amidohydrolase & octa_29980 \\
\hline Alcohol dehydrogenase, class IV & octa_30040 \\
\hline $\begin{array}{r}\text { carbohydrate } \mathrm{ABC} \text { transporter substrate-binding protein, } \\
\text { CUT1 family (TC 3.A.1.1.-) }\end{array}$ & octa_30070 \\
\hline $\begin{array}{r}\text { carbohydrate } \mathrm{ABC} \text { transporter membrane protein } 2, \\
\text { CUT1 family }\end{array}$ & octa_30090 \\
\hline $\begin{array}{r}\text { carbohydrate } \mathrm{ABC} \text { transporter membrane protein 1, } \\
\text { CUT1 family }\end{array}$ & octa_30100 \\
\hline glycerol transport system ATP-binding protein & octa_30110 \\
\hline glycerol transport system ATP-binding protein & octa_30120 \\
\hline
\end{tabular}


Table A.2: continued

\begin{tabular}{|c|c|}
\hline DESCRIPTION & LOCUS TAG \\
\hline glycerol transport system permease protein & octa_30150 \\
\hline glycerol transport system permease protein & octa_3016o \\
\hline dihydroxyacetone kinase & octa_30190 \\
\hline multiple sugar transport system ATP-binding protein & octa_30200 \\
\hline $\begin{array}{r}\text { carbohydrate ABC transporter ATP-binding protein, } \\
\text { CUT1 family }\end{array}$ & octa_30210 \\
\hline $\begin{array}{r}\text { carbohydrate } \mathrm{ABC} \text { transporter membrane protein 2, } \\
\text { CUT1 family }\end{array}$ & octa_30230 \\
\hline $\begin{array}{r}\text { carbohydrate } \mathrm{ABC} \text { transporter membrane protein } 1, \\
\text { CUT1 family }\end{array}$ & octa_30240 \\
\hline $\begin{array}{r}\text { carbohydrate } \mathrm{ABC} \text { transporter substrate-binding protein, } \\
\text { CUT1 family }\end{array}$ & octa_30250 \\
\hline LacI family transcriptional regulator & octa_3026o \\
\hline pseudoazurin & octa_30490 \\
\hline simple sugar transport system permease protein & octa_306oo \\
\hline simple sugar transport system substrate-binding protein & octa_30610 \\
\hline transcriptional regulator, TetR family & octa_3260o \\
\hline hypothetical protein & octa_32620 \\
\hline transcriptional regulator, RpiR family & octa_32640 \\
\hline Predicted N-formylglutamate amidohydrolase & octa_3265o \\
\hline $\begin{array}{l}\text { TRAP-type mannitol/chloroaromatic compound } \\
\text { transport system, small permease component }\end{array}$ & octa_3266o \\
\hline TRAP transporter, DctM subunit & octa_32670 \\
\hline $\begin{array}{r}\text { Tat (twin-arginine translocation) pathway signal } \\
\text { sequence }\end{array}$ & octa_3268o \\
\hline glutamine synthetase & octa_3269o \\
\hline Acyl-CoA reductase & octa_32700 \\
\hline 4-hydroxybutyrate dehydrogenase & octa_32710 \\
\hline $\begin{array}{r}\text { spermidine/putrescine transport system } \\
\text { substrate-binding protein }\end{array}$ & octa_32720 \\
\hline $\begin{array}{r}\text { spermidine/putrescine transport system ATP-binding } \\
\text { protein }\end{array}$ & octa_32730 \\
\hline $\begin{array}{r}\text { spermidine/putrescine transport system permease } \\
\text { protein }\end{array}$ & octa_32740 \\
\hline $\begin{array}{r}\text { spermidine/putrescine transport system permease } \\
\text { protein }\end{array}$ & octa_32750 \\
\hline phosphate transport system permease protein & octa_36790 \\
\hline ribose transport system permease protein & octa_37100 \\
\hline $\begin{array}{r}\text { NAD(P)-dependent dehydrogenase, short-chain alcohol } \\
\text { dehydrogenase family }\end{array}$ & octa_37170 \\
\hline C4-dicarboxylate transporter, DctM subunit & octa_37210 \\
\hline
\end{tabular}


Table A.2: continued

\begin{tabular}{|c|c|}
\hline DESCRIPTION & LOCUS TAG \\
\hline Transposase zinc-binding domain-containing protein & octa_38930 \\
\hline Transposase zinc-binding domain-containing protein & octa_3896o \\
\hline Methyltransferase domain-containing protein & octa_40100 \\
\hline methylthioribose-1-phosphate isomerase & octa_41790 \\
\hline L-fuculose-phosphate aldolase & octa_41810 \\
\hline Pyridoxamine $5^{\prime}$-phosphate oxidase & octa_41850 \\
\hline Site-specific DNA recombinase & octa_42090 \\
\hline
\end{tabular}

Table A.3: Genes exclusive to the group formed by O. ascidiaceicola, O. temperatus (group II, Section 3.2), as determined by po2group_stats.pl. For each specific OG, the annotation and LOCUS TAG in the genome of O. ascidiaceicola is provided for reference.

\begin{tabular}{|c|c|}
\hline DESCRIPTION & LOCUS TAG \\
\hline hypothetical protein & oasc_o0150 \\
\hline hypothetical protein & oasc_00540 \\
\hline hypothetical protein & oasc_0056o \\
\hline Exopolysaccharide synthesis, ExoD & oasc_02050 \\
\hline hypothetical protein & oasc_02120 \\
\hline hypothetical protein & oasc_02260 \\
\hline hypothetical protein & oasc_03570 \\
\hline SnoaL-like domain protein & oasc_04030 \\
\hline Stress response protein NhaX & oasc_o4570 \\
\hline hypothetical protein & oasc_04790 \\
\hline Protein MtfA & oasc_05940 \\
\hline hypothetical protein & oasc_07250 \\
\hline Glycosyl transferases group 1 & oasc_07940 \\
\hline hypothetical protein & oasc_07950 \\
\hline $\begin{array}{r}\text { N-acetylglucosaminyl-diphospho-decaprenol } \\
\text { L-rhamnosyltransferase }\end{array}$ & oasc_07990 \\
\hline hypothetical protein & oasc_o8ooo \\
\hline hypothetical protein & oasc_08010 \\
\hline hypothetical protein & oasc_08020 \\
\hline Sulfotransferase domain protein & oasc_08030 \\
\hline hypothetical protein & oasc_o8070 \\
\hline Bifunctional hemolysin/adenylate cyclase precursor & oasc_0809o \\
\hline MAPEG family protein & oasc_08820 \\
\hline PilZ domain protein & oasc_og68o \\
\hline hypothetical protein & oasc_09750 \\
\hline
\end{tabular}


Table A.3: continued

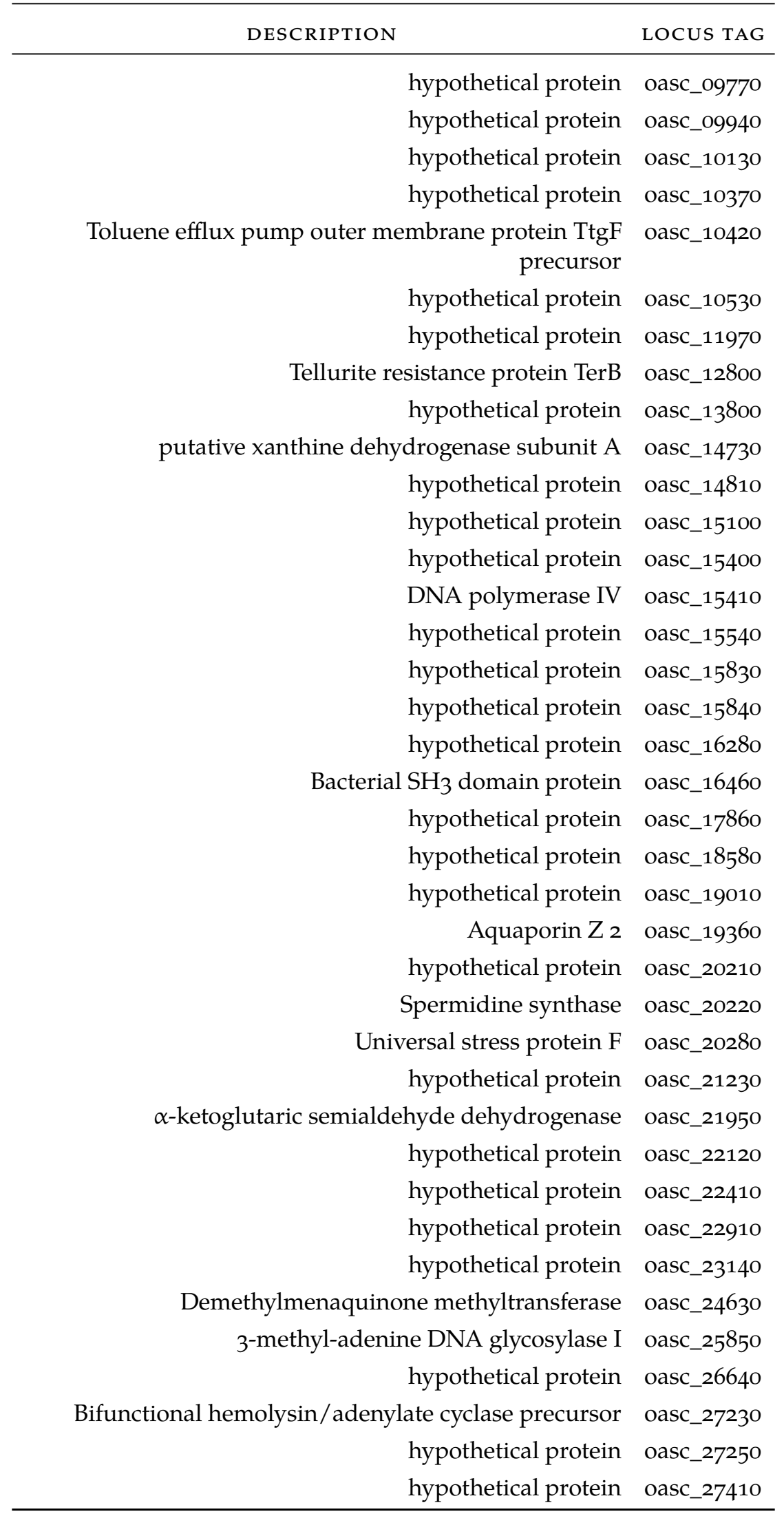


Table A.3: continued

\begin{tabular}{rr}
\hline DESCRIPTION & LOCUS TAG \\
\hline Sensor protein EvgS precursor & oasc_28280 \\
hypothetical protein & oasc_28300 \\
Poly-beta-1,6-N-acetyl-D-glucosamine synthase & oasc_28310 \\
Phytanoyl-CoA dioxygenase (PhyH) & oasc_30060 \\
hypothetical protein & oasc_30790 \\
hypothetical protein & oasc_32470 \\
Antitoxin ParD4 & oasc_32670 \\
\hline
\end{tabular}

Table A.4: Genes exclusive to the group formed by P. jejudonensis and isolate $\mathrm{NH}_{9}-\mathrm{P}_{7}$ (group III, Section 3.2), as determined by po2group_stats.pl. For each specific OG, the annotation and LOCUS TAG in the genome of $P$. jejudonensis is provided for reference.

\begin{tabular}{rr}
\hline DESCRIPTION & LOCUS TAG \\
\hline Protein of unknown function (DUF3833) & OJEJ_00230 \\
Catechol 2,3-dioxygenase & OJEJ_02950 \\
magnesium chelatase accessory protein & OJEJ_03060 \\
magnesium chelatase subunit D & OJEJ_03070 \\
magnesium chelatase subunit I & OJEJ_03080 \\
spheroidene monooxygenase & OJEJ_03090 \\
phytoene synthase & OJEJ_03110 \\
TspO and MBR related proteins & OJEJ_03120 \\
carotenoid 1,2-hydratase & OJEJ_03130 \\
1-hydroxycarotenoid 3,4-desaturase & OJEJ_03140 \\
farnesyl-diphosphate synthase & OJEJ_03150 \\
demethylspheroidene O-methyltransferase & OJEJ_03160 \\
3-hydroxyethyl bacteriochlorophyllide a dehydrogenase & OJEJ_03170 \\
chlorophyllide a reductase subunit X & OJEJ_03180 \\
chlorophyllide a reductase subunit Y & OJEJ_03200 \\
chlorophyllide a reductase subunit Z & OJEJ_03210 \\
PufQ cytochrome subunit & OJEJ_03220 \\
light-harvesting complex 1 beta chain & OJEJ_03230 \\
light-harvesting complex 1 alpha chain & OJEJ_03240 \\
photosynthetic reaction center L subunit & OJEJ_03250 \\
photosynthetic reaction center M subunit & OJEJ_03260 \\
Intrinsic membrane protein PufX & OJEJ_03270 \\
g-deoxy-D-xylulose-5hosphate synthase & OJEJ_03290 \\
& OJEJ_03310 \\
\hline
\end{tabular}


Table A.4: continued

\begin{tabular}{|c|c|}
\hline DESCRIPTION & LOCUS TAG \\
\hline MFS transporter, BCD family, chlorophyll transporter & OJEJ_O3320 \\
\hline chlorophyll synthase & OJEJ_03330 \\
\hline transcriptional regulator PpsR & OJEJ_03340 \\
\hline Methanogenic corrinoid protein $\mathrm{MtbC} 1$ & OJEJ_03350 \\
\hline 3-vinyl bacteriochlorophyllide hydratase & OJEJ_03360 \\
\hline ferredoxin protochlorophyllide reductase subunit $\mathrm{N}$ & OJEJ_03370 \\
\hline ferredoxin protochlorophyllide reductase subunit B & OJEJ_03380 \\
\hline cobaltochelatase CobN subunit & OJEJ_03390 \\
\hline ferredoxin protochlorophyllide reductase subunit L & OJEJ_03410 \\
\hline Mg-protoporphyrin IX methyltransferase & OJEJ_03420 \\
\hline MFS transporter, BCD family, chlorophyll transporter & OJEJ_03430 \\
\hline photosynthetic reaction center $\mathrm{H}$ subunit & OJEJ_03440 \\
\hline PH domain-containing protein & OJEJ_03450 \\
\hline putative photosynthetic complex assembly protein & OJEJ_03460 \\
\hline hypothetical protein & OJEJ_03470 \\
\hline $\begin{array}{r}\text { Mg-protoporphyrin IX monomethyl ester (oxidative) } \\
\text { cyclase }\end{array}$ & OJEJ_03480 \\
\hline putative photosynthetic complex assembly protein 2 & OJEJ_03490 \\
\hline dimethylglycine dehydrogenase & OJEJ_03590 \\
\hline $\begin{array}{r}\text { Threonine/homoserine/homoserine lactone efflux } \\
\text { protein }\end{array}$ & OJEJ_04100 \\
\hline divinylchlorophyllide 8-vinylreductase & OJEJ_04740 \\
\hline CubicO group peptidase, $\beta$-lactamase class $C$ family & OJEJ_04920 \\
\hline single-strand binding protein & OJEJ_07520 \\
\hline hypothetical protein & OJEJ_08130 \\
\hline $\begin{array}{r}\text { TRAP-type C4-dicarboxylate transport system, } \\
\text { substrate-binding protein }\end{array}$ & OJEJ_08520 \\
\hline $\begin{array}{r}\text { Tripartite ATP-independent transporter, DctQ } \\
\text { component }\end{array}$ & OJEJ_08530 \\
\hline $\begin{array}{r}\text { Hemolysin-type calcium-binding repeat-containing } \\
\text { protein }\end{array}$ & OJEJ_09550 \\
\hline DNA-binding transcriptional regulator, LysR family & OJEJ_10900 \\
\hline cold shock protein (beta-ribbon, CspA family) & OJEJ_11070 \\
\hline hypothetical protein & OJEJ_12280 \\
\hline cytochrome b561 & OJEJ_12290 \\
\hline hypothetical protein & OJEJ_12550 \\
\hline hypothetical protein & OJEJ_13220 \\
\hline hypothetical protein & OJEJ_13230 \\
\hline malonyl-CoA/methylmalonyl-CoA synthetase & OJEJ_13850 \\
\hline protein-tyrosine phosphatase & OJEJ_14330 \\
\hline
\end{tabular}


Table A.4: continued

\begin{tabular}{|c|c|}
\hline DESCRIPTION & LOCUS TAG \\
\hline NAD-dependent deacetylase & OJEJ_14340 \\
\hline divinyl protochlorophyllide a 8-vinyl-reductase & OJEJ_14840 \\
\hline monoamine oxidase & OJEJ_1548o \\
\hline hypothetical protein & OJEJ_16740 \\
\hline hypothetical protein & OJEJ_16750 \\
\hline $\begin{array}{r}\text { Predicted Zn-dependent protease, minimal } \\
\text { metalloprotease (MMP)-like domain }\end{array}$ & OJEJ_16950 \\
\hline Long-chain fatty acid transport protein & OJEJ_1788o \\
\hline hypothetical protein & OJEJ_18740 \\
\hline hypothetical protein & OJEJ_21600 \\
\hline hypothetical protein & OJEJ_22280 \\
\hline hypothetical protein & OJEJ_23970 \\
\hline alpha-glucosidase & OJEJ_26210 \\
\hline GDP-mannose 4,6-dehydratase & OJEJ_28540 \\
\hline UPFo271 protein & OJEJ_29170 \\
\hline inhibitor of KinA & OJEJ_29180 \\
\hline diguanylate cyclase (GGDEF) domain-containing protein & OJEJ_30050 \\
\hline $\begin{array}{r}\text { Uncharacterized conserved protein YndB, AHSA1/ } \\
\text { START domain }\end{array}$ & OJEJ_30720 \\
\hline hypothetical protein & OJEJ_3288o \\
\hline starvation-inducible DNA-binding protein & OJEJ_33070 \\
\hline
\end{tabular}

Table A.5: Genes exclusive to the Octadecabacter-associated isolate E8 (group IV, Section 3.2), as determined by po2group_stats.pl. For each specific $O G$, the annotation and LOCUS TAG in the genome is provided for reference.

\begin{tabular}{|c|c|}
\hline DESCRIPTION & LOCUS TAG \\
\hline Nucleoside-diphosphate-sugar epimerase & Gaoo68414_1011 \\
\hline $\begin{array}{r}\text { peptide/nickel transport system ATP-binding } \\
\text { protein }\end{array}$ & Gaoo68414_1012 \\
\hline $\begin{array}{r}\text { peptide/nickel transport system ATP-binding } \\
\text { protein }\end{array}$ & Gaoo68414_1013 \\
\hline Multidrug resistance protein & Gaoo68414_1021 \\
\hline transcriptional regulator, LacI family & Gaoo68414_1024 \\
\hline Phage terminase large subunit (GpA) & Gaoo68414_1025 \\
\hline $\begin{array}{r}\text { Phage DNA packaging protein, Nu1 subunit of } \\
\text { terminase }\end{array}$ & Gaoo68414_1026 \\
\hline $\begin{array}{r}\text { Uncharacterized conserved protein YjdB, contains } \\
\text { Ig-like domain }\end{array}$ & Gaoo68414_1031 \\
\hline
\end{tabular}


Table A.5: continued

\begin{tabular}{|c|c|}
\hline DESCRIPTION & LOCUS TAG \\
\hline Phage minor tail protein $U$ & Gaoo68414_10310 \\
\hline Phage minor tail protein $U$ & Gaoo68414_1032 \\
\hline Prophage minor tail protein Z (GPZ) & Gaoo68414_1033 \\
\hline Phage Head-Tail Attachment & Gaoo68414_1034 \\
\hline DNA packaging protein FI & Gaoo68414_1035 \\
\hline Phage major capsid protein $\mathrm{E}$ & Gaoo68414_1036 \\
\hline Bacteriophage lambda head decoration protein $\mathrm{D}$ & Gaoo68414_1037 \\
\hline $\begin{array}{r}\text { protein C (EC:3.4.21.69). Serine peptidase. MEROPS } \\
\text { family S49 }\end{array}$ & Gaoo68414_1038 \\
\hline $\begin{array}{r}\text { Uncharacterized conserved protein YjdB, contains } \\
\text { Ig-like domain }\end{array}$ & Gaoo68414_1039 \\
\hline hypothetical protein & Gaoo68414_10410 \\
\hline peptide/nickel transport system permease protein & Gaoo68414_1042 \\
\hline $\begin{array}{r}\text { peptide/nickel transport system substrate-binding } \\
\text { protein }\end{array}$ & Gaoo68414_1043 \\
\hline $\begin{array}{r}\text { oligopeptide transport system substrate-binding } \\
\text { protein }\end{array}$ & Gaoo68414_1044 \\
\hline oligopeptide transport system permease protein & Gaoo68414_1047 \\
\hline oligopeptide transport system permease protein & Gaoo68414_1048 \\
\hline $\begin{array}{r}\text { transcriptional regulator, } \mathrm{XRE} \text { family with cupin } \\
\text { sensor }\end{array}$ & Gaoo68414_1049 \\
\hline adenosylhomocysteinase & Gaoo68414_10525 \\
\hline hypothetical protein & Gaoo68414_1055 \\
\hline C-terminal domain of 1-Cys peroxiredoxin & Gaoo68414_1061 \\
\hline $\begin{array}{r}\text { NitT/TauT family transport system ATP-binding } \\
\text { protein }\end{array}$ & Gaoo68414_10610 \\
\hline $\begin{array}{r}\text { NitT/TauT family transport system permease } \\
\text { protein }\end{array}$ & Gaoo68414_10611 \\
\hline $\begin{array}{r}\text { NitT/TauT family transport system permease } \\
\text { protein }\end{array}$ & Gaoo68414_10612 \\
\hline MgsA AAA+ ATPase C terminal & Gaoo68414_10613 \\
\hline Nucleoside 2-deoxyribosyltransferase & Gaoo68414_10614 \\
\hline regulatory protein, lacI family & Gaoo68414_10615 \\
\hline transcriptional regulator, GntR family & Gaoo68414_10616 \\
\hline $\begin{array}{r}\text { NitT/TauT family transport system permease } \\
\text { protein }\end{array}$ & Gaoo68414_10617 \\
\hline $\begin{array}{r}\text { NitT/TauT family transport system ATP-binding } \\
\text { protein }\end{array}$ & Gaoo68414_10618 \\
\hline $\begin{array}{r}\text { NitT/TauT family transport system } \\
\text { substrate-binding protein }\end{array}$ & Gaoo68414_10619 \\
\hline hypothetical protein & Gaoo68414_1062 \\
\hline
\end{tabular}


Table A.5: continued

\begin{tabular}{|c|c|}
\hline DESCRIPTION & LOCUS TAG \\
\hline fumarylpyruvate hydrolase & Gaoo68414_1062C \\
\hline 4-hydroxy-4-methyl-2-oxoglutarate aldolase & Gaoo68414_10621 \\
\hline 3-hydroxyisobutyrate dehydrogenase & Gaoo68414_10622 \\
\hline Uncharacterized membrane protein $\mathrm{YfcA}$ & Gaoo68414_10623 \\
\hline $\mathrm{ABC}$ transporter & Gaoo68414_10625 \\
\hline hypothetical protein & Gaoo68414_10626 \\
\hline chromosome partitioning protein, ParB family & Gaoo68414_10627 \\
\hline chromosome partitioning protein & Gaoo68414_10628 \\
\hline hypothetical protein & Gaoo68414_1063 \\
\hline DDE domain-containing protein & Gaoo68414_10631 \\
\hline hypothetical protein & Gaoo68414_10632 \\
\hline iron(III) transport system ATP-binding protein & Gaoo68414_10633 \\
\hline iron(III) transport system substrate-binding protein & Gaoo68414_10634 \\
\hline iron(III) transport system permease protein & Gaoo68414_10635 \\
\hline 4-nitrophenyl phosphatase & Gaoo68414_10636 \\
\hline peptide/nickel transport system permease protein & Gaoo68414_10638 \\
\hline $\begin{array}{r}\text { Type I phosphodiesterase / nucleotide } \\
\text { pyrophosphatase }\end{array}$ & Gaoo68414_10639 \\
\hline ADP-ribose pyrophosphatase & Gaoo68414_1064 \\
\hline glycerophosphoryl diester phosphodiesterase & Gaoo68414_10640 \\
\hline oligopeptide transport system ATP-binding protein & Gaoo68414_10641 \\
\hline $\begin{array}{r}\text { peptide/nickel transport system substrate-binding } \\
\text { protein }\end{array}$ & Gaoo68414_10643 \\
\hline catalase & Gaoo68414_1065 \\
\hline hypothetical protein & Gaoo68414_10654 \\
\hline hypothetical protein & Gaoo68414_10655 \\
\hline Transposase InsO and inactivated derivatives & Gaoo68414_10656 \\
\hline GntR family transcriptional regulator & Gaoo68414_10657 \\
\hline basic membrane protein $\mathrm{A}$ & Gaoo68414_10658 \\
\hline simple sugar transport system ATP-binding protein & Gaoo68414_10659 \\
\hline simple sugar transport system permease protein & Gaoo68414_1066c \\
\hline nucleoside $\mathrm{ABC}$ transporter membrane protein & Gaoo68414_10661 \\
\hline Nicotinamidase-related amidase & Gaoo68414_10662 \\
\hline ribokinase & Gaoo68414_10663 \\
\hline uridine phosphorylase & Gaoo68414_10664 \\
\hline $\begin{array}{l}\text { Uncharacterized protein, contains SIS (Sugar } \\
\text { ISomerase) phosphosugar binding domain }\end{array}$ & Gaoo68414_10665 \\
\hline $\begin{array}{l}\text { succinate-semialdehyde dehydrogenase / } \\
\text { glutarate-semialdehyde dehydrogenase }\end{array}$ & Gaoo68414_10666 \\
\hline hypothetical protein & Gaoo68414_10667 \\
\hline
\end{tabular}


Table A.5: continued

\begin{tabular}{|c|c|}
\hline DESCRIPTION & LOCUS TAG \\
\hline transcriptional regulator, $\mathrm{RpiR}$ family & Gaoo68414_1068 \\
\hline $\begin{array}{r}\text { ABC-type nitrate/sulfonate/bicarbonate transport } \\
\text { system, substrate-binding protein }\end{array}$ & Gaoo68414_1069 \\
\hline Helix-turn-helix & Gaoo68414_1071 \\
\hline hypothetical protein & Gaoo68414_10710 \\
\hline Integrase core domain-containing protein & Gaoo68414_10711 \\
\hline hypothetical protein & Gaoo68414_10712 \\
\hline Uracil DNA glycosylase superfamily protein & Gaoo68414_10713 \\
\hline hypothetical protein & Gaoo68414_1072 \\
\hline hypothetical protein & Gaoo68414_1073 \\
\hline HYR domain-containing protein & Gaoo68414_1074 \\
\hline hypothetical protein & Gaoo68414_1075 \\
\hline hypothetical protein & Gaoo68414_1076 \\
\hline Putative flagellar system-associated repeat & Gaoo68414_1077 \\
\hline Site-specific recombinase XerD & Gaoo68414_10771 \\
\hline ParB-like nuclease domain-containing protein & Gaoo68414_10772 \\
\hline hypothetical protein & Gaoo68414_10773 \\
\hline Site-specific DNA recombinase & Gaoo68414_10774 \\
\hline hypothetical protein & Gaoo68414_10775 \\
\hline protein of unknown function (DUF4102) & Gaoo68414_10776 \\
\hline Integrase core domain-containing protein & Gaoo68414_10777 \\
\hline $\begin{array}{r}\text { Hemolysin-type calcium-binding repeat-containing } \\
\text { protein }\end{array}$ & Gaoo68414_10778 \\
\hline hypothetical protein & Gaoo68414_1078 \\
\hline hypothetical protein & Gaoo68414_1079 \\
\hline hypothetical protein & Gaoo68414_1081 \\
\hline putative efflux protein, MATE family & Gaoo68414_10812 \\
\hline hypothetical protein & Gaoo68414_10813 \\
\hline cephalosporin-C deacetylase & Gaoo68414_10814 \\
\hline transcriptional regulator, LacI family & Gaoo68414_10815 \\
\hline FAD dependent oxidoreductase & Gaoo68414_10816 \\
\hline $\begin{array}{r}\text { multiple sugar transport system substrate-binding } \\
\text { protein }\end{array}$ & Gaoo68414_10817 \\
\hline multiple sugar transport system permease protein & Gaoo68414_10818 \\
\hline multiple sugar transport system permease protein & Gaoo68414_10819 \\
\hline $\begin{array}{r}\text { multiple sugar transport system ATP-binding } \\
\text { protein }\end{array}$ & Gaoo68414_10820 \\
\hline Phosphoglycerate dehydrogenase & Gaoo68414_10821 \\
\hline hypothetical protein & Gaoo68414_10825 \\
\hline Signal transduction histidine kinase & Gaoo68414_10826 \\
\hline
\end{tabular}


Table A.5: continued

\begin{tabular}{|c|c|}
\hline DESCRIPTION & LOCUS TAG \\
\hline hypothetical protein & Gaoo68414_10827 \\
\hline hypothetical protein & Gaoo68414_10828 \\
\hline Phage integrase family & Gaoo68414_10829 \\
\hline hypothetical protein & Gaoo68414_1083 \\
\hline hypothetical protein & Gaoo68414_10830 \\
\hline hypothetical protein & Gaoo68414_10831 \\
\hline hypothetical protein & Gaoo68414_10832 \\
\hline Phage integrase family protein & Gaoo68414_10833 \\
\hline methyltransferase, FkbM family & Gaoo68414_1084 \\
\hline Glycosyl transferase family 2 & Gaoo68414_1085 \\
\hline PRC-barrel domain-containing protein & Gaoo68414_10850 \\
\hline $\begin{array}{r}\text { Glycosyltransferase sugar-binding region containing } \\
\text { DXD motif-containing protein }\end{array}$ & Gaoo68414_1086 \\
\hline Glycosyltransferase involved in cell wall bisynthesis & Gaoo68414_1087 \\
\hline hypothetical protein & Gaoo68414_10875 \\
\hline Phage integrase family protein & Gaoo68414_10876 \\
\hline hypothetical protein & Gaoo68414_10877 \\
\hline hypothetical protein & Gaoo68414_10878 \\
\hline Transposase & Gaoo68414_10879 \\
\hline hypothetical protein & Gaoo68414_1088 \\
\hline putative RNA 2'-phosphotransferase & Gaoo68414_1088o \\
\hline AraC-type DNA-binding protein & Gaoo68414_10884 \\
\hline $\begin{array}{r}\text { outer membrane autotransporter barrel } \\
\text { domain-containing protein }\end{array}$ & Gaoo68414_10885 \\
\hline hypothetical protein & Gaoo68414_10886 \\
\hline transporter family-2 protein & Gaoo68414_10887 \\
\hline Acetyltransferase (GNAT) family protein & Gaoo68414_10888 \\
\hline Helix-turn-helix domain-containing protein & Gaoo68414_10889 \\
\hline Integrase core domain-containing protein & Gaoo68414_1089 \\
\hline hypothetical protein & Gaoo68414_1089o \\
\hline hypothetical protein & Gaoo68414_10891 \\
\hline protein of unknown function (DUF4422) & Gaoo68414_1091 \\
\hline oligopeptide transport system ATP-binding protein & Gaoo68414_109100 \\
\hline oligopeptide transport system ATP-binding protein & Gaoo68414_109101 \\
\hline peptide/nickel transport system permease protein & Gaoo68414_109103 \\
\hline $\begin{array}{r}\text { peptide/nickel transport system substrate-binding } \\
\text { protein }\end{array}$ & Gaoo68414_109104 \\
\hline Short-chain dehydrogenase & Gaoo68414_109106 \\
\hline DNA-binding transcriptional regulator, MarR family & Gaoo68414_109107 \\
\hline hypothetical protein & Gaoo68414_109114 \\
\hline
\end{tabular}


Table A.5: continued

\begin{tabular}{|c|c|}
\hline DESCRIPTION & LOCUS TAG \\
\hline hypothetical protein & Gaoo68414_109115 \\
\hline hypothetical protein & Gaoo68414_109116 \\
\hline Methyltransferase domain-containing protein & Gaoo68414_109117 \\
\hline hypothetical protein & Gaoo68414_109118 \\
\hline High-affinity nickel-transport protein & Gaoo68414_109122 \\
\hline $\begin{array}{r}\text { ABC-type uncharacterized transport system, } \\
\text { substrate-binding protein }\end{array}$ & Gaoo68414_109123 \\
\hline hypothetical protein & Gaoo68414_109128 \\
\hline $\begin{array}{r}\text { TRAP-type } \mathrm{C}_{4} \text {-dicarboxylate transport system, small } \\
\text { permease component }\end{array}$ & Gaoo68414_109130 \\
\hline $\mathrm{C}_{4}$-dicarboxylate transporter, DctM subunit & Gaoo68414_109131 \\
\hline hypothetical protein & Gaoo68414_109132 \\
\hline $\begin{array}{r}\text { amino acid ABC transporter ATP-binding protein, } \\
\text { PAAT family }\end{array}$ & Gaoo68414_109133 \\
\hline $\begin{array}{r}\text { amino acid ABC transporter membrane protein 2, } \\
\text { PAAT family (TC 3.A.1.3.-) }\end{array}$ & Gaoo68414_109134 \\
\hline $\begin{array}{r}\text { amino acid } \mathrm{ABC} \text { transporter membrane protein } 1, \\
\text { PAAT family }\end{array}$ & Gaoo68414_109135 \\
\hline $\begin{array}{r}\text { amino acid ABC transporter substrate-binding } \\
\text { protein, PAAT family }\end{array}$ & Gaoo68414_109136 \\
\hline hypothetical protein & Gaoo68414_109142 \\
\hline creatinine amidohydrolase & Gaoo68414_109146 \\
\hline Uncharacterized membrane protein & Gaoo68414_109148 \\
\hline transcriptional regulator, LacI family & Gaoo68414_109149 \\
\hline cytidine deaminase & Gaoo68414_109150 \\
\hline $\begin{array}{r}\text { 5-methylthioadenosine/S-adenosylhomocysteine } \\
\text { deaminase }\end{array}$ & Gaoo68414_109151 \\
\hline non-specific riboncleoside hydrolase & Gaoo68414_109152 \\
\hline nucleoside-binding protein & Gaoo68414_109153 \\
\hline simple sugar transport system permease protein & Gaoo68414_109155 \\
\hline simple sugar transport system permease protein & Gaoo68414_109156 \\
\hline guanine deaminase & Gaoo68414_109157 \\
\hline UDP-glucose 4-epimerase & Gaoo68414_10916o \\
\hline Glycosyltransferase involved in cell wall bisynthesis & Gaoo68414_109161 \\
\hline hypothetical protein & Gaoo68414_109162 \\
\hline Sulfotransferase family protein & Gaoo68414_109163 \\
\hline Polysaccharide pyruvyl transferase & Gaoo68414_109165 \\
\hline Glycosyl transferase family 2 & Gaoo68414_109166 \\
\hline Glycosyltransferase involved in cell wall bisynthesis & Gaoo68414_109167 \\
\hline hypothetical protein & Gaoo68414_109168 \\
\hline
\end{tabular}


Table A.5: continued

\begin{tabular}{|c|c|}
\hline DESCRIPTION & LOCUS TAG \\
\hline $\begin{array}{r}\text { Lipopolysaccharide biosynthesis protein, } \\
\text { LPS:glycosyltransferase }\end{array}$ & Gaoo68414_109169 \\
\hline $\begin{array}{r}\text { Endonuclease/Exonuclease/phosphatase family } \\
\text { protein }\end{array}$ & Gaoo68414_10917 \\
\hline Glycosyltransferase, GT2 family & Gaoo68414_1092 \\
\hline TctA family transporter & Gaoo68414_10930 \\
\hline putative tricarboxylic transport membrane protein & Gaoo68414_10931 \\
\hline $\begin{array}{r}\text { Tripartite-type tricarboxylate transporter, receptor } \\
\text { component TctC }\end{array}$ & Gaoo68414_10932 \\
\hline hypothetical protein & Gaoo68414_10933 \\
\hline $\begin{array}{r}\text { LysR family transcriptional regulator, cys regulon } \\
\text { transcriptional activator }\end{array}$ & Gaoo68414_10934 \\
\hline phosphate uptake regulator, $\mathrm{PhoU}$ & Gaoo68414_10939 \\
\hline hypothetical protein & Gaoo68414_10951 \\
\hline toxin $\mathrm{CcdB}$ & Gaoo68414_10952 \\
\hline $\begin{array}{l}\text { LysR family transcriptional regulator, glycine } \\
\text { cleavage system transcriptional activator }\end{array}$ & Gaoo68414_10953 \\
\hline hypothetical protein & Gaoo68414_10954 \\
\hline protein of unknown function (DUF885) & Gaoo68414_10955 \\
\hline glyoxylate/hydroxypyruvate reductase A & Gaoo68414_10956 \\
\hline $\begin{array}{r}\text { peptide/nickel transport system substrate-binding } \\
\text { protein }\end{array}$ & Gaoo68414_10957 \\
\hline peptide/nickel transport system permease protein & Gaoo68414_10959 \\
\hline $\begin{array}{r}\text { Hemolysin-type calcium-binding repeat-containing } \\
\text { protein }\end{array}$ & Gaoo68414_1096 \\
\hline $\begin{array}{r}\text { peptide/nickel transport system ATP-binding } \\
\text { protein }\end{array}$ & Gaoo68414_1096o \\
\hline $\begin{array}{l}\text { peptidase T. Metallo peptidase. MEROPS family } \\
\text { M2oB }\end{array}$ & Gaoo68414_10961 \\
\hline hypothetical protein & Gaoo68414_10962 \\
\hline ketopantoate reductase & Gaoo68414_10963 \\
\hline Predicted dehydrogenase & Gaoo68414_10964 \\
\hline $\begin{array}{r}\text { peptide/nickel transport system substrate-binding } \\
\text { protein }\end{array}$ & Gaoo68414_10967 \\
\hline peptide/nickel transport system permease protein & Gaoo68414_10968 \\
\hline hypothetical protein & Gaoo68414_10970 \\
\hline $\begin{array}{r}\text { peptide/nickel transport system ATP-binding } \\
\text { protein }\end{array}$ & Gaoo68414_10971 \\
\hline $\begin{array}{r}\text { peptide/nickel transport system ATP-binding } \\
\text { protein }\end{array}$ & Gaoo68414_10972 \\
\hline peptide/nickel transport system permease protein & Gaoo68414_10973 \\
\hline
\end{tabular}


Table A.5: continued

\begin{tabular}{|c|c|}
\hline DESCRIPTION & LOCUS TAG \\
\hline peptide/nickel transport system permease protein & Gaoo68414_10974 \\
\hline $\begin{array}{r}\text { peptide/nickel transport system substrate-binding } \\
\text { protein }\end{array}$ & Gaoo68414_10975 \\
\hline $\begin{array}{r}\text { Nucleotide-binding universal stress protein, UspA } \\
\text { family }\end{array}$ & Gaoo68414_10981 \\
\hline TRAP transporter, DctM subunit & Gaoo68414_10982 \\
\hline $\begin{array}{r}\text { TRAP-type } \mathrm{C}_{4} \text {-dicarboxylate transport system, small } \\
\text { permease component }\end{array}$ & Gaoo68414_10983 \\
\hline $\begin{array}{r}\text { TRAP-type C4-dicarboxylate transport system, } \\
\text { substrate-binding protein }\end{array}$ & Gaoo68414_10984 \\
\hline amidohydrolase & Gaoo68414_10989 \\
\hline gluconate 2-dehydrogenase gamma chain & Gaoo68414_10990 \\
\hline gluconate 2-dehydrogenase alpha chain & Gaoo68414_10991 \\
\hline Cytochrome c, mono- and diheme variants & Gaoo68414_10992 \\
\hline putative membrane protein & Gaoo68414_10993 \\
\hline Uncharacterized membrane protein & Gaoo68414_10994 \\
\hline Uncharacterized membrane protein & Gaoo68414_10995 \\
\hline cytochrome c oxidase subunit 2 & Gaoo68414_10996 \\
\hline cytochrome c oxidase subunit I+III & Gaoo68414_10997 \\
\hline hypothetical protein & Gaoo68414_10998 \\
\hline Glycine/D-amino acid oxidase (deaminating) & Gaoo68414_10999 \\
\hline hypothetical protein & Gaoo68414_110106 \\
\hline TupA-like ATPgrasp & Gaoo68414_110107 \\
\hline Integrase core domain-containing protein & Gaoo68414_11011 \\
\hline MJoo42 family finger-like domain-containing protein & Gaoo68414_110114 \\
\hline hypothetical protein & Gaoo68414_110119 \\
\hline Helix-turn-helix domain-containing protein & Gaoo68414_11012 \\
\hline transcriptional regulator, AraC family & Gaoo68414_110122 \\
\hline hypothetical protein & Gaoo68414_110137 \\
\hline YrhK-like protein & Gaoo68414_110139 \\
\hline hypothetical protein & Gaoo68414_11014 \\
\hline Opacity protein & Gaoo68414_110145 \\
\hline hypothetical protein & Gaoo68414_110148 \\
\hline alcohol dehydrogenase & Gaoo68414_11015 \\
\hline Ankyrin repeat-containing protein & Gaoo68414_110158 \\
\hline hypothetical protein & Gaoo68414_11016 \\
\hline hypothetical protein & Gaoo68414_11018 \\
\hline transcriptional regulator, LysR family & Gaoo68414_11019 \\
\hline hypothetical protein & Gaoo68414_110194 \\
\hline $\mathrm{Cu}^{+}$-exporting ATPase & Gaoo68414_110205 \\
\hline
\end{tabular}


Table A.5: continued

\begin{tabular}{|c|c|}
\hline DESCRIPTION & LOCUS TAG \\
\hline solute: $\mathrm{Na}^{+}$symporter, SSS family & Gaoo68414_11022 \\
\hline Protein N-acetyltransferase, RimJ/RimL family & Gaoo68414_110220 \\
\hline hypothetical protein & Gaoo68414_11023 \\
\hline hypothetical protein & Gaoo68414_110236 \\
\hline hypothetical protein & Gaoo68414_11024 \\
\hline hypothetical protein & Gaoo68414_110244 \\
\hline hypothetical protein & Gaoo68414_11026 \\
\hline hypothetical protein & Gaoo68414_110272 \\
\hline hypothetical protein & Gaoo68414_110275 \\
\hline Predicted dehydrogenase & Gaoo68414_11028 \\
\hline aldehyde dehydrogenase & Gaoo68414_110289 \\
\hline hypothetical protein & Gaoo68414_11029 \\
\hline hypothetical protein & Gaoo68414_110291 \\
\hline PAS domain S-box-containing protein & Gaoo68414_110292 \\
\hline hypothetical protein & Gaoo68414_110294 \\
\hline Lipoprotein-anchoring transpeptidase ErfK/SrfK & Gaoo68414_110295 \\
\hline hypothetical protein & Gaoo68414_110296 \\
\hline DNA-binding transcriptional regulator, MerR family & Gaoo68414_110297 \\
\hline zinc transporter, ZIP family & Gaoo68414_110298 \\
\hline hypothetical protein & Gaoo68414_110299 \\
\hline hypothetical protein & Gaoo68414_1103 \\
\hline hypothetical protein & Gaoo68414_11030 \\
\hline Alpha/beta hydrolase family protein & Gaoo68414_110300 \\
\hline hypothetical protein & Gaoo68414_110301 \\
\hline DNA-binding transcriptional regulator, MarR family & Gaoo68414_110302 \\
\hline YHYH protein & Gaoo68414_110303 \\
\hline hypothetical protein & Gaoo68414_110304 \\
\hline $\begin{array}{r}\text { MFS transporter, DHA1 family, bicyclomycin/ } \\
\text { chloramphenicol resistance protein }\end{array}$ & Gaoo68414_110305 \\
\hline intein N-terminal splicing region & Gaoo68414_110309 \\
\hline hypothetical protein & Gaoo68414_11031 \\
\hline hypothetical protein & Gaoo68414_110312 \\
\hline $\begin{array}{r}\text { iron complex transport system substrate-binding } \\
\text { protein }\end{array}$ & Gaoo68414_110313 \\
\hline hypothetical protein & Gaoo68414_110314 \\
\hline $\begin{array}{r}\text { iron complex transport system substrate-binding } \\
\text { protein }\end{array}$ & Gaoo68414_110315 \\
\hline $\begin{array}{r}\text { iron complex transport system substrate-binding } \\
\text { protein }\end{array}$ & Gaoo68414_110319 \\
\hline
\end{tabular}


Table A.5: continued

\begin{tabular}{|c|c|}
\hline DESCRIPTION & LOCUS TAG \\
\hline $\begin{array}{r}\text { iron complex transport system substrate-binding } \\
\text { protein }\end{array}$ & Gaoo68414_110320 \\
\hline iron complex transport system permease protein & Gaoo68414_110321 \\
\hline iron complex transport system permease protein & Gaoo68414_110322 \\
\hline iron complex transport system ATP-binding protein & Gaoo68414_110323 \\
\hline Coiled stalk of trimeric autotransporter adhesin & Gaoo68414_110324 \\
\hline Invasion protein IalB, involved in pathogenesis & Gaoo68414_110325 \\
\hline hypothetical protein & Gaoo68414_110326 \\
\hline hypothetical protein & Gaoo68414_110327 \\
\hline transcriptional regulator, ArsR family & Gaoo68414_110332 \\
\hline hypothetical protein & Gaoo68414_110333 \\
\hline uncharacterized protein & Gaoo68414_110334 \\
\hline hypothetical protein & Gaoo68414_110335 \\
\hline hypothetical protein & Gaoo68414_110336 \\
\hline hypothetical protein & Gaoo68414_110337 \\
\hline protein of unknown function (DUF2088) & Gaoo68414_110338 \\
\hline altronate hydrolase & Gaoo68414_110339 \\
\hline L-lactate dehydrogenase & Gaoo68414_110341 \\
\hline $\begin{array}{r}\text { Tripartite-type tricarboxylate transporter, receptor } \\
\text { component TctC }\end{array}$ & Gaoo68414_110343 \\
\hline $\begin{array}{r}\text { Tripartite tricarboxylate transporter TctB family } \\
\text { protein }\end{array}$ & Gaoo68414_110344 \\
\hline putative tricarboxylic transport membrane protein & Gaoo68414_110345 \\
\hline DNA-binding transcriptional regulator, GntR family & Gaoo68414_110346 \\
\hline Integrase core domain-containing protein & Gaoo68414_110347 \\
\hline hypothetical protein & Gaoo68414_110348 \\
\hline aromatic-amino-acid transaminase & Gaoo68414_11035 \\
\hline hypothetical protein & Gaoo68414_11039 \\
\hline hypothetical protein & Gaoo68414_1104 \\
\hline hypothetical protein & Gaoo68414_11041 \\
\hline hypothetical protein & Gaoo68414_11043 \\
\hline Protein of unknown function (DUF1328) & Gaoo68414_11045 \\
\hline hypothetical protein & Gaoo68414_1105 \\
\hline transcriptional regulator, LacI family & Gaoo68414_11052 \\
\hline hypothetical protein & Gaoo68414_1107 \\
\hline hypothetical protein & Gaoo68414_1108 \\
\hline YcxB-like protein & Gaoo68414_11080 \\
\hline hypothetical protein & Gaoo68414_11098 \\
\hline Site-specific recombinase XerD & Gaoo68414_1111 \\
\hline type I restriction enzyme M protein & Gaoo68414_11110 \\
\hline
\end{tabular}


Table A.5: continued

\begin{tabular}{|c|c|}
\hline & S TAG \\
\hline MFS transporter, SET family, sugar efflux transporter & Gaoo68414_111107 \\
\hline $\begin{array}{r}\text { Excalibur calcium-binding domain-containing } \\
\text { protein }\end{array}$ & Gaoo68414_111110 \\
\hline $\mathrm{Ca}^{2+}$-binding protein, $\mathrm{RTX}$ toxin-related & Gaoo68414_111118 \\
\hline hypothetical protein & Gaoo68414_111126 \\
\hline hypothetical protein & Gaoo68414_11113 \\
\hline single-strand binding protein & Gaoo68414_111164 \\
\hline $\begin{array}{r}\text { Uncharacterized conserved protein YbjQ, UPFo145 } \\
\text { family }\end{array}$ & Gaoo68414_111189 \\
\hline Site-specific recombinase XerD & Gaoo68414_1112 \\
\hline Tetratricopeptide repeat-containing protein & Gaoo68414_111202 \\
\hline hypothetical protein & Gaoo68414_111215 \\
\hline hypothetical protein & Gaoo68414_111217 \\
\hline Predicted dehydrogenase & Gaoo68414_111218 \\
\hline glucose-fructose oxidore & 4_111219 \\
\hline $\begin{array}{r}\text { monosaccharide } \mathrm{ABC} \text { transporter membrane } \\
\text { protein, } \mathrm{CUT}_{2} \text { family }\end{array}$ & Gaoo68414_111220 \\
\hline $\begin{array}{r}\text { monosaccharide } \mathrm{ABC} \text { transporter membrane } \\
\text { protein, } \mathrm{CUT}_{2} \text { family }\end{array}$ & Gaoo68414_111221 \\
\hline $\begin{array}{r}\text { monosaccharide } \mathrm{ABC} \text { transporter ATP-binding } \\
\text { protein, } \mathrm{CUT} 2 \text { family }\end{array}$ & Gaoo68414_111222 \\
\hline $\begin{array}{r}\text { monosaccharide } \mathrm{ABC} \text { transporter substrate-binding } \\
\text { protein, } \mathrm{CUT} 2 \text { family }\end{array}$ & Gaoo68414_111223 \\
\hline Sugar phosphate isomerase/epimerase & Gaoo68414_111224 \\
\hline Predicted dehydrogenase & Gaoo68414_111225 \\
\hline regulatory protein, lacI family & Gaoo68414_111226 \\
\hline $\begin{array}{r}\text { substrate-binding protein domain-containing } \\
\text { protein }\end{array}$ & Gaoo68414_111227 \\
\hline Inosine-uridine nucleoside N-ribohydrolase & Gaoo68414_111228 \\
\hline $\begin{array}{r}\text { putative spermidine/putrescine transport system } \\
\text { permease protein }\end{array}$ & Gaoo68414_111229 \\
\hline $\begin{array}{r}\text { putative spermidine/putrescine transport system } \\
\text { permease protein }\end{array}$ & Gaoo68414_111230 \\
\hline $\begin{array}{r}\text { putative spermidine/putrescine transport system } \\
\text { substrate-binding protein }\end{array}$ & Gaoo68414_111231 \\
\hline L-fucose isomerase & Gaoo68414_111233 \\
\hline GntR family transcriptional regulator & Gaoo68414_111234 \\
\hline phenylacetaldehyde dehydrogenase & Gaoo68414_111235 \\
\hline tagatose 1,6 -diphosphate aldolase & Gaoo68414_111236 \\
\hline dihydroxyacetone kinase DhaK subunit & Gaoo68414_111237 \\
\hline dihydroxyacetone kinase DhaL subunit & Gaoo68414_111238 \\
\hline
\end{tabular}


Table A.5: continued

\begin{tabular}{|c|c|}
\hline DESCRIPTION & LOCUS TAG \\
\hline D-psicose/D-tagatose/L-ribulose 3-epimerase & Gaoo68414_111239 \\
\hline acetyl-CoA synthetase & Gaoo68414_111240 \\
\hline Rubredoxin-like zinc ribbon domain (DUF35 N) & Gaoo68414_111241 \\
\hline acetyl-CoA C-acetyltransferase & Gaoo68414_111242 \\
\hline Major Facilitator Superfamily protein & Gaoo68414_111243 \\
\hline $\begin{array}{r}\text { gamma-glutamyltranspeptidase / glutathione } \\
\text { hydrolase }\end{array}$ & Gaoo68414_111244 \\
\hline D-3-phosphoglycerate dehydrogenase & Gaoo68414_111245 \\
\hline $\begin{array}{r}\text { Hemolysin-type calcium-binding repeat-containing } \\
\text { protein }\end{array}$ & Gaoo68414_111247 \\
\hline iron complex transport system ATP-binding protein & Gaoo68414_111249 \\
\hline $\begin{array}{r}\text { iron complex transport system substrate-binding } \\
\text { protein }\end{array}$ & Gaoo68414_111251 \\
\hline$(2 \mathrm{Fe}-2 \mathrm{~S})$ ferredoxin & Gaoo68414_111252 \\
\hline hypothetical protein & Gaoo68414_111254 \\
\hline regulatory protein, luxR family & Gaoo68414_111256 \\
\hline hypothetical protein & Gaoo68414_111257 \\
\hline hypothetical protein & Gaoo68414_111258 \\
\hline hypothetical protein & Gaoo68414_111261 \\
\hline hypothetical protein & Gaoo68414_111262 \\
\hline hypothetical protein & Gaoo68414_111263 \\
\hline hypothetical protein & Gaoo68414_111264 \\
\hline Plasmid recombination enzyme & Gaoo68414_111265 \\
\hline hypothetical protein & Gaoo68414_111266 \\
\hline hypothetical protein & Gaoo68414_111267 \\
\hline hypothetical protein & Gaoo68414_111268 \\
\hline Signal transduction histidine kinase & Gaoo68414_111269 \\
\hline PAS domain S-box-containing protein & Gaoo68414_111270 \\
\hline hypothetical protein & Gaoo68414_111279 \\
\hline hypothetical protein & Gaoo68414_111288 \\
\hline hypothetical protein & Gaoo68414_111291 \\
\hline hypothetical protein & Gaoo68414_1113 \\
\hline glutathione S-transferase & Gaoo68414_111311 \\
\hline hypothetical protein & Gaoo68414_111313 \\
\hline DNA-binding transcriptional regulator, IscR family & Gaoo68414_111314 \\
\hline Thioredoxin reductase & Gaoo68414_111315 \\
\hline FAD/FMN-containing dehydrogenase & Gaoo68414_111316 \\
\hline EcoRII C terminal & Gaoo68414_111317 \\
\hline $\begin{array}{r}\text { putative spermidine/putrescine transport system } \\
\text { permease protein }\end{array}$ & Gaoo68414_111319 \\
\hline
\end{tabular}


Table A.5: continued

\begin{tabular}{|c|c|}
\hline DESCRIPTION & LOCUS TAG \\
\hline $\begin{array}{r}\text { putative spermidine/putrescine transport system } \\
\text { permease protein }\end{array}$ & Gaoo68414_111320 \\
\hline $\begin{array}{r}\text { putative spermidine/putrescine transport system } \\
\text { substrate-binding protein }\end{array}$ & Gaoo68414_111321 \\
\hline hypothetical protein & Gaoo68414_111323 \\
\hline hypothetical protein & Gaoo68414_111324 \\
\hline transcriptional regulator, IclR family & Gaoo68414_111325 \\
\hline acetolactate synthase- $1 / 2 / 3$ large subunit & Gaoo68414_111326 \\
\hline transcriptional regulator, LacI family & Gaoo68414_111330 \\
\hline hypothetical protein & Gaoo68414_111332 \\
\hline hypothetical protein & Gaoo68414_111333 \\
\hline adenosylhomocysteinase & Gaoo68414_111334 \\
\hline adenosylhomocysteinase & Gaoo68414_111335 \\
\hline hypothetical protein & Gaoo68414_1114 \\
\hline hypothetical protein & Gaoo68414_1115 \\
\hline Site-specific DNA recombinase & Gaoo68414_1116 \\
\hline hypothetical protein & Gaoo68414_1117 \\
\hline hypothetical protein & Gaoo68414_11177 \\
\hline type I restriction enzyme, $\mathrm{R}$ subunit & Gaoo68414_1118 \\
\hline type I restriction enzyme, S subunit & Gaoo68414_1119 \\
\hline hypothetical protein & Gaoo68414_11197 \\
\hline Transposase and inactivated derivatives & Gaoo68414_1121 \\
\hline hypothetical protein & Gaoo68414_11210 \\
\hline zinc transporter, ZIP family & Gaoo68414_112122 \\
\hline hypothetical protein & Gaoo68414_112135 \\
\hline DNA-binding transcriptional regulator, MerR family & Gaoo68414_112136 \\
\hline intein $\mathrm{N}$-terminal splicing region & Gaoo68414_112137 \\
\hline $\begin{array}{r}\text { Uncharacterized conserved protein YdeI, YjbR/ } \\
\text { CyaY-like superfamily, DUF1801 family }\end{array}$ & Gaoo68414_112138 \\
\hline $\begin{array}{r}\text { Ubiquinone/menaquinone biosynthesis } \\
\text { C-methylase UbiE }\end{array}$ & Gaoo68414_11215 \\
\hline $\begin{array}{l}\text { Lrp/AsnC family transcriptional regulator, } \\
\text { leucine-responsive regulatory protein }\end{array}$ & Gaoo68414_112153 \\
\hline leucine dehydrogenase & Gaoo68414_112154 \\
\hline Uncharacterized conserved protein, DUF1330 family & Gaoo68414_112155 \\
\hline hypothetical protein & Gaoo68414_112156 \\
\hline NADPH2:quinone reductase & Gaoo68414_112157 \\
\hline Uncharacterized conserved protein, DUF1697 family & Gaoo68414_112158 \\
\hline hypothetical protein & Gaoo68414_112159 \\
\hline
\end{tabular}


Table A.5: continued

\begin{tabular}{|c|c|}
\hline DESCRIPTION & LOCUS TAG \\
\hline $\begin{array}{r}\text { Alpha/beta hydrolase family/Bacterial regulatory } \\
\text { proteins, luxR family }\end{array}$ & Gaoo68414_112162 \\
\hline hypothetical protein & Gaoo68414_112185 \\
\hline transposase & Gaoo68414_1122 \\
\hline Activator of Hsp9o ATPase homolog 1-like protein & Gaoo68414_112213 \\
\hline Methyladenine glycosylase & Gaoo68414_112215 \\
\hline Transposase IS20o like & Gaoo68414_112220 \\
\hline Abortive infection C-terminus & Gaoo68414_112221 \\
\hline Cell Wall Hydrolase & Gaoo68414_112243 \\
\hline Ribosomal protein $\mathrm{S} 18$ acetylase RimI & Gaoo68414_112251 \\
\hline ribosome-associated heat shock protein Hsp15 & Gaoo68414_112269 \\
\hline Zn-dependent dipeptidase, dipeptidase homolog & Gaoo68414_112275 \\
\hline hypothetical protein & Gaoo68414_112299 \\
\hline hypothetical protein & Gaoo68414_1123 \\
\hline hypothetical protein & Gaoo68414_112327 \\
\hline hypothetical protein & Gaoo68414_112332 \\
\hline hypothetical protein & Gaoo68414_112336 \\
\hline Uncharacterized membrane protein & Gaoo68414_112352 \\
\hline hypothetical protein & Gaoo68414_112356 \\
\hline hypothetical protein & Gaoo68414_112362 \\
\hline hypothetical protein & Gaoo68414_112364 \\
\hline peptide/nickel transport system permease protein & Gaoo68414_112370 \\
\hline regulatory protein, luxR family & Gaoo68414_112372 \\
\hline $\begin{array}{r}\text { Aldehyde oxidase and xanthine dehydrogenase, } a / b \\
\text { hammerhead domain }\end{array}$ & Gaoo68414_112383 \\
\hline Site-specific recombinase XerD & Gaoo68414_112397 \\
\hline hypothetical protein & Gaoo68414_112398 \\
\hline Helix-turn-helix & Gaoo68414_112399 \\
\hline Protein of unknown function $\left(\mathrm{DUF}_{37} 68\right)$ & Gaoo68414_1124 \\
\hline hypothetical protein & Gaoo68414_11240o \\
\hline hypothetical protein & Gaoo68414_112401 \\
\hline sporadically distributed protein, TIGRo4141 family & Gaoo68414_112402 \\
\hline Phage integrase family protein & Gaoo68414_112404 \\
\hline hypothetical protein & Gaoo68414_112405 \\
\hline hypothetical protein & Gaoo68414_112406 \\
\hline hypothetical protein & Gaoo68414_112407 \\
\hline hypothetical protein & Gaoo68414_112408 \\
\hline replication region DNA-binding $\mathrm{N}$-term & Gaoo68414_112409 \\
\hline plasmid mobilization system relaxase & Gaoo68414_112410 \\
\hline
\end{tabular}


Table A.5: continued

\begin{tabular}{|c|c|}
\hline DESCRIPTION & LOCUS TAG \\
\hline hypothetical protein & Gaoo68414_112411 \\
\hline hypothetical protein & Gaoo68414_112412 \\
\hline TRAP transporter, $4 \mathrm{TM} / 12 \mathrm{TM}$ fusion protein & Gaoo68414_112413 \\
\hline Dienelactone hydrolase & Gaoo68414_112414 \\
\hline glutathione S-transferase & Gaoo68414_112415 \\
\hline peptide/nickel transport system permease protein & Gaoo68414_112421 \\
\hline hypothetical protein & Gaoo68414_112459 \\
\hline hypothetical protein & Gaoo68414_112462 \\
\hline hypothetical protein & Gaoo68414_112498 \\
\hline hypothetical protein & Gaoo68414_11251 \\
\hline hypothetical protein & Gaoo68414_112510 \\
\hline hypothetical protein & Gaoo68414_11252 \\
\hline Peptidase M5oB-like & Gaoo68414_112558 \\
\hline$A B C-2$ type transport system permease protein & Gaoo68414_112561 \\
\hline$A B C-2$ type transport system permease protein & Gaoo68414_112562 \\
\hline HlyD family secretion protein & Gaoo68414_112563 \\
\hline transcriptional regulator, TetR family & Gaoo68414_112564 \\
\hline regulatory helix-turn-helix protein, lysR family & Gaoo68414_112565 \\
\hline hypothetical protein & Gaoo68414_112566 \\
\hline Uncharacterized lipoprotein YbaY & Gaoo68414_112567 \\
\hline hypothetical protein & Gaoo68414_112568 \\
\hline Integrase core domain-containing protein & Gaoo68414_112569 \\
\hline Helix-turn-helix domain-containing protein & Gaoo68414_112570 \\
\hline hypothetical protein & Gaoo68414_112571 \\
\hline hypothetical protein & Gaoo68414_112572 \\
\hline transposase & Gaoo68414_112573 \\
\hline hypothetical protein & Gaoo68414_112575 \\
\hline hypothetical protein & Gaoo68414_112576 \\
\hline HupE / UreJ protein & Gaoo68414_112577 \\
\hline hypothetical protein & Gaoo68414_112578 \\
\hline hypothetical protein & Gaoo68414_112579 \\
\hline hypothetical protein & Gaoo68414_112580 \\
\hline Transposase DDE domain-containing protein & Gaoo68414_112581 \\
\hline hypothetical protein & Gaoo68414_112582 \\
\hline Patatin-like phospholipase & Gaoo68414_112583 \\
\hline hypothetical protein & Gaoo68414_112584 \\
\hline DinB family protein & Gaoo68414_112585 \\
\hline Aldo/keto reductase & Gaoo68414_112586 \\
\hline luciferase-type oxidoreductase, BA3436 family & Gaoo68414_112587 \\
\hline
\end{tabular}


Table A.5: continued

\begin{tabular}{|c|c|}
\hline DESCRIPTION & LOCUS TAG \\
\hline Predicted arabinose efflux permease, MFS family & Gaoo68414_112588 \\
\hline DNA-binding transcriptional regulator, LysR family & Gaoo68414_112589 \\
\hline hypothetical protein & Gaoo68414_112590 \\
\hline Tetratricopeptide repeat-containing protein & Gaoo68414_112591 \\
\hline hypothetical protein & Gaoo68414_112592 \\
\hline hypothetical protein & Gaoo68414_112593 \\
\hline TniQ & Gaoo68414_112594 \\
\hline hypothetical protein & Gaoo68414_112595 \\
\hline TniQ protein & Gaoo68414_112596 \\
\hline AAA domain-containing protein & Gaoo68414_112597 \\
\hline putative transposase & Gaoo68414_112598 \\
\hline hypothetical protein & Gaoo68414_112599 \\
\hline Site-specific recombinase XerD & Gaoo68414_1126 \\
\hline hypothetical protein & Gaoo68414_11260o \\
\hline HTH-like domain-containing protein & Gaoo68414_1126o3 \\
\hline hypothetical protein & Gaoo68414_112604 \\
\hline outer membrane protein, adhesin transport system & Gaoo68414_112605 \\
\hline membrane fusion protein, adhesin transport system & Gaoo68414_112606 \\
\hline ATP-binding cassette, subfamily C, LapB & Gaoo68414_112607 \\
\hline hypothetical protein & Gaoo68414_1126o8 \\
\hline hypothetical protein & Gaoo68414_11263 \\
\hline hypothetical protein & Gaoo68414_1127 \\
\hline hypothetical protein & Gaoo68414_1128 \\
\hline $\begin{array}{l}\text { Uncaracterized surface protein containing fasciclin } \\
\text { (FAS1) repeats }\end{array}$ & Gaoo68414_11281 \\
\hline hypothetical protein & Gaoo68414_11285 \\
\hline hypothetical protein & Gaoo68414_1129 \\
\hline hypothetical protein & Gaoo68414_1131 \\
\hline HYR domain-containing protein & Gaoo68414_11310 \\
\hline hypothetical protein & Gaoo68414_1131000 \\
\hline hypothetical protein & Gaoo68414_1131005 \\
\hline hypothetical protein & Gaoo68414_113101 \\
\hline uncharacterized protein & Gaoo68414_1131019 \\
\hline $\begin{array}{r}\text { BFD-like [2Fe-2S] binding domain-containing } \\
\text { protein }\end{array}$ & Gaoo68414_1131024 \\
\hline hypothetical protein & Gaoo68414_1131026 \\
\hline hypothetical protein & Gaoo68414_1131027 \\
\hline hypothetical protein & Gaoo68414_1131028 \\
\hline $\begin{array}{r}\text { solute carrier family } 34 \text { (sodium-dependent } \\
\text { phosphate cotransporter) }\end{array}$ & Gaoo68414_1131029 \\
\hline
\end{tabular}


Table A.5: continued

\begin{tabular}{|c|c|}
\hline DESCRIPTION & LOCUS TAG \\
\hline hypothetical protein & Gaoo68414_113103 \\
\hline DNA-binding transcriptional regulator, LysR family & Gaoo68414_1131030 \\
\hline transcriptional regulator, IclR family & Gaoo68414_1131037 \\
\hline putative tricarboxylic transport membrane protein & Gaoo68414_1131038 \\
\hline $\begin{array}{r}\text { Tripartite tricarboxylate transporter TctB family } \\
\text { protein }\end{array}$ & Gaoo68414_1131039 \\
\hline $\begin{array}{r}\text { Crotonobetainyl-CoA:carnitine CoA-transferase } \\
\text { CaiB }\end{array}$ & Gaoo68414_1131041 \\
\hline citrate lyase subunit beta / citryl-CoA lyase & Gaoo68414_1131042 \\
\hline transcriptional regulator, LacI family & Gaoo68414_1131043 \\
\hline $\begin{array}{r}\text { putative aldouronate transport system permease } \\
\text { protein }\end{array}$ & Gaoo68414_1131044 \\
\hline $\begin{array}{r}\text { putative aldouronate transport system permease } \\
\text { protein }\end{array}$ & Gaoo68414_1131045 \\
\hline $\begin{array}{r}\text { putative aldouronate transport system } \\
\text { substrate-binding protein }\end{array}$ & Gaoo68414_1131046 \\
\hline ADP-ribosylglycohydrolase & Gaoo68414_1131047 \\
\hline ADP-ribosylglycohydrolase & Gaoo68414_1131048 \\
\hline $\begin{array}{r}\text { multiple sugar transport system ATP-binding } \\
\text { protein }\end{array}$ & Gaoo68414_1131049 \\
\hline Predicted amidohydrolase & Gaoo68414_113106o \\
\hline SIR2-like domain-containing protein & Gaoo68414_1131066 \\
\hline hypothetical protein & Gaoo68414_1131068 \\
\hline hypothetical protein & Gaoo68414_113107 \\
\hline Homeodomain-like domain-containing protein & Gaoo68414_1131071 \\
\hline transposase & Gaoo68414_1131072 \\
\hline EcoRII C terminal & Gaoo68414_1131073 \\
\hline $\begin{array}{r}\text { DNA-binding transcriptional response regulator, } \\
\text { NtrC family, contains REC, AAA-type ATPase, and a } \\
\text { Fis-type DNA-binding domains }\end{array}$ & Gaoo68414_1131076 \\
\hline two-component system, NtrC family, sensor kinase & Gaoo68414_1131077 \\
\hline Major Facilitator Superfamily protein & Gaoo68414_1131078 \\
\hline lysine 2-monooxygenase (EC 1.13.12.2) & Gaoo68414_1131079 \\
\hline sulfopropanediol 3-dehydrogenase & Gaoo68414_1131082 \\
\hline hypothetical protein & Gaoo68414_1131086 \\
\hline Winged helix-turn helix & Gaoo68414_11311 \\
\hline hypothetical protein & Gaoo68414_1131102 \\
\hline hypothetical protein & Gaoo68414_113111 \\
\hline $\mathrm{BCCT}$, betaine/carnitine/choline family transporter & Gaoo68414_1131120 \\
\hline hypothetical protein & Gaoo68414_1131125 \\
\hline hypothetical protein & Gaoo68414_1131153 \\
\hline
\end{tabular}


Table A.5: continued

\begin{tabular}{|c|c|}
\hline DESCRIPTION & LOCUS TAG \\
\hline hypothetical protein & Gaoo68414_113116 \\
\hline hypothetical protein & Gaoo68414_1131164 \\
\hline hypothetical protein & Gaoo68414_1131175 \\
\hline Site-specific recombinase XerD & Gaoo68414_1131177 \\
\hline hypothetical protein & Gaoo68414_1131178 \\
\hline Predicted phosphoesterase, NUDIX family & Gaoo68414_1131179 \\
\hline Methyltransferase domain-containing protein & Gaoo68414_113118o \\
\hline hypothetical protein & Gaoo68414_1131181 \\
\hline hypothetical protein & Gaoo68414_1131183 \\
\hline hypothetical protein & Gaoo68414_1131184 \\
\hline hypothetical protein & Gaoo68414_1131185 \\
\hline hypothetical protein & Gaoo68414_1131186 \\
\hline hypothetical protein & Gaoo68414_1131187 \\
\hline hypothetical protein & Gaoo68414_1131188 \\
\hline $\mathrm{N}$-acetylmuramoyl-L-alanine amidase & Gaoo68414_1131189 \\
\hline Membrane-associated phospholipid phosphatase & Gaoo68414_113119 \\
\hline hypothetical protein & Gaoo68414_1131190 \\
\hline hypothetical protein & Gaoo68414_1131191 \\
\hline hypothetical protein & Gaoo68414_1131192 \\
\hline Endonuclease YncB, thermonuclease family & Gaoo68414_1131193 \\
\hline $\begin{array}{r}\text { Phosphatidylserine/ } \\
\text { phosphatidylglycerophosphate/cardiolipin } \\
\text { synthase }\end{array}$ & Gaoo68414_1131195 \\
\hline FAD-NAD(P)-binding & Gaoo68414_1131196 \\
\hline Uncharacterized conserved protein & Gaoo68414_1131197 \\
\hline Transposase InsO and inactivated derivatives & Gaoo68414_11312 \\
\hline $\begin{array}{r}\text { FMN-dependent oxidoreductase, nitrilotriacetate } \\
\text { monooxygenase family }\end{array}$ & Gaoo68414_1131205 \\
\hline $\begin{array}{l}\text { Predicted metal-dependent enzyme of the } \\
\text { double-stranded beta helix superfamily }\end{array}$ & Gaoo68414_1131210 \\
\hline DNA-binding transcriptional regulator, LysR family & Gaoo68414_1131211 \\
\hline $\begin{array}{r}\text { His Kinase A (phospho-acceptor) } \\
\text { domain-containing protein }\end{array}$ & Gaoo68414_1131216 \\
\hline PAS domain S-box-containing protein & Gaoo68414_1131217 \\
\hline $\begin{array}{r}\text { Response regulator receiver domain-containing } \\
\text { protein }\end{array}$ & Gaoo68414_1131218 \\
\hline RND family efflux transporter, MFP subunit & Gaoo68414_1131219 \\
\hline hypothetical protein & Gaoo68414_113122 \\
\hline $\begin{array}{r}\text { hydrophobe/amphiphile efflux-1 (HAE1) family } \\
\text { protein }\end{array}$ & Gaoo68414_1131220 \\
\hline
\end{tabular}


Table A.5: continued

\begin{tabular}{|c|c|}
\hline DESCRIPTION & LOCUS TAG \\
\hline hypothetical protein & Gaoo68414_1131221 \\
\hline transcriptional regulator, TetR family & Gaoo68414_1131231 \\
\hline $\begin{array}{r}\text { Predicted flavoprotein } \mathrm{CzcO} \text { associated with the } \\
\text { cation diffusion facilitator } \mathrm{CzcD}\end{array}$ & Gaoo68414_1131232 \\
\hline bile acid: $\mathrm{Na}^{+}$symporter, BASS family & Gaoo68414_1131233 \\
\hline UDP-glucose 4-epimerase & Gaoo68414_1131234 \\
\hline Short-chain dehydrogenase & Gaoo68414_1131235 \\
\hline hypothetical protein & Gaoo68414_113125 \\
\hline hypothetical protein & Gaoo68414_1131250 \\
\hline hypothetical protein & Gaoo68414_1131259 \\
\hline hypothetical protein & Gaoo68414_1131262 \\
\hline sec-independent protein translocase protein TatB & Gaoo68414_1131290 \\
\hline Integrase core domain-containing protein & Gaoo68414_11313 \\
\hline hypothetical protein & Gaoo68414_1131306 \\
\hline TIGR02300 family protein & Gaoo68414_113131 \\
\hline dihydroorotase & Gaoo68414_1131312 \\
\hline hypothetical protein & Gaoo68414_1131315 \\
\hline $\begin{array}{r}\text { MFS transporter, DHA1 family, bicyclomycin/ } \\
\text { chloramphenicol resistance protein }\end{array}$ & Gaoo68414_1131317 \\
\hline hypothetical protein & Gaoo68414_1131320 \\
\hline $\begin{array}{r}\text { Uncharacterized membrane protein YoaK, UPFo7oo } \\
\text { family }\end{array}$ & Gaoo68414_1131366 \\
\hline hypothetical protein & Gaoo68414_11314 \\
\hline hypothetical protein & Gaoo68414_1131400 \\
\hline hypothetical protein & Gaoo68414_1131401 \\
\hline hypothetical protein & Gaoo68414_1131402 \\
\hline Sulfotransferase domain-containing protein & Gaoo68414_1131404 \\
\hline hypothetical protein & Gaoo68414_113142 \\
\hline hypothetical protein & Gaoo68414_1131428 \\
\hline hypothetical protein & Gaoo68414_113147 \\
\hline hypothetical protein & Gaoo68414_1131470 \\
\hline hypothetical protein & Gaoo68414_1131471 \\
\hline $\begin{array}{r}\text { gamma-glutamyltranspeptidase / glutathione } \\
\text { hydrolase }\end{array}$ & Gaoo68414_1131491 \\
\hline zinc transport system substrate-binding protein & Gaoo68414_1131494 \\
\hline SIR2-like domain-containing protein & Gaoo68414_11315 \\
\hline $\mathrm{Ca}^{2+}$-binding protein, $\mathrm{RTX}$ toxin-related & Gaoo68414_1131502 \\
\hline hypothetical protein & Gaoo68414_1131503 \\
\hline hypothetical protein & Gaoo68414_1131504 \\
\hline regulatory protein, luxR family & Gaoo68414_1131505 \\
\hline
\end{tabular}


Table A.5: continued

\begin{tabular}{|c|c|}
\hline DESCRIPTION & LOCUS TAG \\
\hline hypothetical protein & Gaoo68414_1131506 \\
\hline hypothetical protein & Gaoo68414_1131508 \\
\hline FAD binding domain-containing protein & Gaoo68414_1131509 \\
\hline Cytochrome C oxidase, cbb3-type, subunit III & Gaoo68414_1131510 \\
\hline transcriptional regulator, TetR family & Gaoo68414_1131511 \\
\hline $\begin{array}{r}\text { polar amino acid transport system substrate-binding } \\
\text { protein }\end{array}$ & Gaoo68414_1131513 \\
\hline polar amino acid transport system permease protein & Gaoo68414_1131514 \\
\hline $\begin{array}{r}\text { polar amino acid transport system ATP-binding } \\
\text { protein }\end{array}$ & Gaoo68414_1131515 \\
\hline methionine-gamma-lyase & Gaoo68414_1131516 \\
\hline hypothetical protein & Gaoo68414_1131521 \\
\hline Phage integrase family & Gaoo68414_1131522 \\
\hline hypothetical protein & Gaoo68414_1131561 \\
\hline DNA-binding protein HU-alpha & Gaoo68414_1131563 \\
\hline hypothetical protein & Gaoo68414_1131593 \\
\hline $\begin{array}{r}\text { Hemolysin-type calcium-binding repeat-containing } \\
\text { protein }\end{array}$ & Gaoo68414_1131594 \\
\hline hypothetical protein & Gaoo68414_1131595 \\
\hline $\begin{array}{l}\text { LysR family transcriptional regulator, glycine } \\
\text { cleavage system transcriptional activator }\end{array}$ & Gaoo68414_1131596 \\
\hline hypothetical protein & Gaoo68414_1131598 \\
\hline ferredoxin, $2 \mathrm{Fe}-2 \mathrm{~S}$ & Gaoo68414_1131599 \\
\hline trk system potassium uptake protein TrkH & Gaoo68414_11316 \\
\hline $\begin{array}{r}\text { Predicted metal-dependent hydrolase, TIM-barrel } \\
\text { fold }\end{array}$ & Gaoo68414_11316o1 \\
\hline transcriptional regulator, LysR family & Gaoo68414_1131603 \\
\hline $\begin{array}{r}\text { Enamine deaminase RidA, house cleaning of } \\
\text { reactive enamine intermediates, YjgF/YERo57c/ } \\
\text { UK114 family }\end{array}$ & Gaoo68414_1131605 \\
\hline D-arabinitol 4-dehydrogenase & Gaoo68414_113162 \\
\hline transcriptional regulator, AraC family & Gaoo68414_113163 \\
\hline Glycosyl hydrolase 108 & Gaoo68414_1131642 \\
\hline hypothetical protein & Gaoo68414_1131666 \\
\hline hypothetical protein & Gaoo68414_1131684 \\
\hline trk system potassium uptake protein TrkA & Gaoo68414_11317 \\
\hline hypothetical protein & Gaoo68414_1131703 \\
\hline $\begin{array}{r}\text { His Kinase A (phospho-acceptor) } \\
\text { domain-containing protein }\end{array}$ & Gaoo68414_1131704 \\
\hline Glycosyltransferase involved in cell wall bisynthesis & Gaoo68414_1131715 \\
\hline hypothetical protein & Gaoo68414_1131720 \\
\hline
\end{tabular}


Table A.5: continued

\begin{tabular}{|c|c|}
\hline DESCRIPTION & LOCUS TAG \\
\hline hypothetical protein & Gaoo68414_1131721 \\
\hline hypothetical protein & Gaoo68414_1131729 \\
\hline $\begin{array}{r}\text { NitT/TauT family transport system } \\
\text { substrate-binding protein }\end{array}$ & Gaoo68414_1131733 \\
\hline transporter, NhaC family & Gaoo68414_1131734 \\
\hline Metallopeptidase family M24 & Gaoo68414_1131735 \\
\hline hypothetical protein & Gaoo68414_1131736 \\
\hline ParD-like antitoxin of type II toxin-antitoxin system & Gaoo68414_1131737 \\
\hline $\begin{array}{r}\text { lactaldehyde dehydrogenase / glycolaldehyde } \\
\text { dehydrogenase }\end{array}$ & Gaoo68414_1131746 \\
\hline hypothetical protein & Gaoo68414_1131747 \\
\hline hypothetical protein & Gaoo68414_1131748 \\
\hline Uncharacterized protein YcnI & Gaoo68414_1131749 \\
\hline hypothetical protein & Gaoo68414_1131750 \\
\hline hypothetical protein & Gaoo68414_1131751 \\
\hline hypothetical protein & Gaoo68414_1131752 \\
\hline hypothetical protein & Gaoo68414_1131754 \\
\hline Integrase core domain-containing protein & Gaoo68414_1131759 \\
\hline hypothetical protein & Gaoo68414_1131760 \\
\hline $\begin{array}{r}\text { transcriptional regulator, XRE family with cupin } \\
\text { sensor }\end{array}$ & Gaoo68414_1131761 \\
\hline $\begin{array}{r}\text { peptide/nickel transport system substrate-binding } \\
\text { protein }\end{array}$ & Gaoo68414_1131762 \\
\hline peptide/nickel transport system permease protein & Gaoo68414_1131764 \\
\hline $\begin{array}{r}\text { ABC-type dipeptide/oligopeptide/nickel transport } \\
\text { system, ATPase component }\end{array}$ & Gaoo68414_1131765 \\
\hline $\begin{array}{r}\text { peptide/nickel transport system ATP-binding } \\
\text { protein }\end{array}$ & Gaoo68414_1131766 \\
\hline GAF domain-containing protein & Gaoo68414_1131767 \\
\hline N-methylhydantoinase A & Gaoo68414_1131768 \\
\hline N-methylhydantoinase B & Gaoo68414_1131769 \\
\hline Xaa-Pro aminopeptidase & Gaoo68414_1131770 \\
\hline N-methylhydantoinase B & Gaoo68414_1131771 \\
\hline hypothetical protein & Gaoo68414_1131772 \\
\hline Integrase core domain-containing protein & Gaoo68414_1131773 \\
\hline hypothetical protein & Gaoo68414_1131774 \\
\hline transposase & Gaoo68414_1131775 \\
\hline $\begin{array}{r}\text { raffinose/stachyose/melibiose transport system } \\
\text { permease protein }\end{array}$ & Gaoo68414_1131777 \\
\hline $\begin{array}{r}\text { raffinose/stachyose/melibiose transport system } \\
\text { permease protein }\end{array}$ & Gaoo68414_1131778 \\
\hline
\end{tabular}


Table A.5: continued

\begin{tabular}{|c|c|}
\hline DESCRIPTION & LOCUS TAG \\
\hline ADP-ribosylglycohydrolase & Gaoo68414_1131779 \\
\hline transcriptional regulator, LacI family & Gaoo68414_1131781 \\
\hline $\begin{array}{r}\text { raffinose/stachyose/melibiose transport system } \\
\text { substrate-binding protein }\end{array}$ & Gaoo68414_1131782 \\
\hline $\begin{array}{r}\text { glucosamine-fructose-6-phosphate } \\
\text { aminotransferase (isomerizing) }\end{array}$ & Gaoo68414_1131783 \\
\hline secondary thiamine-phosphate synthase enzyme & Gaoo68414_1131784 \\
\hline Sugar or nucleoside kinase, ribokinase family & Gaoo68414_1131785 \\
\hline purine nucleosidase & Gaoo68414_1131786 \\
\hline ADP-ribosylglycohydrolase & Gaoo68414_1131787 \\
\hline uridine phosphorylase & Gaoo68414_1131788 \\
\hline hypothetical protein & Gaoo68414_1131789 \\
\hline HAD-superfamily class IIA hydrolase, TIGRo1459 & Gaoo68414_1131791 \\
\hline CDP-alcohol phosphatidyltransferase & Gaoo68414_1131793 \\
\hline $\begin{array}{l}\text { L-glutamine/L-glutamate/L-aspartate/ } \\
\text { L-asparagine ABC transporter membrane protein }\end{array}$ & Gaoo68414_113194 \\
\hline hypothetical protein & Gaoo68414_1132 \\
\hline Sulfotransferase family protein & Gaoo68414_113208 \\
\hline hypothetical protein & Gaoo68414_113212 \\
\hline Hint domain-containing protein & Gaoo68414_113225 \\
\hline hypothetical protein & Gaoo68414_113228 \\
\hline ATPase & Gaoo68414_113234 \\
\hline $\mathrm{Na}^{+} / \mathrm{H}^{+}$-dicarboxylate symporter & Gaoo68414_113244 \\
\hline hypothetical protein & Gaoo68414_113249 \\
\hline $\mathrm{N}$-acylneuraminate cytidylyltransferase & Gaoo68414_113250 \\
\hline hypothetical protein & Gaoo68414_113257 \\
\hline Sulfotransferase family protein & Gaoo68414_11326o \\
\hline serine/threonine protein phosphatase 1 & Gaoo68414_11328 \\
\hline hypothetical protein & Gaoo68414_11329 \\
\hline $\begin{array}{lll}\text { Transposase } & \end{array}$ & Gaoo68414_1133 \\
\hline $\begin{array}{r}\text { ATPase family associated with various cellular } \\
\text { activities (AAA) }\end{array}$ & Gaoo68414_11330 \\
\hline $\begin{array}{r}\text { LPXTG-motif cell wall anchor domain-containing } \\
\text { protein }\end{array}$ & Gaoo68414_113313 \\
\hline $\begin{array}{r}\text { Glutathione-dependent formaldehyde-activating } \\
\text { enzyme }\end{array}$ & Gaoo68414_113327 \\
\hline LPS sulfotransferase NodH & Gaoo68414_113365 \\
\hline hypothetical protein & Gaoo68414_113376 \\
\hline hypothetical protein & Gaoo68414_113377 \\
\hline transposase & Gaoo68414_1134 \\
\hline
\end{tabular}


Table A.5: continued

\begin{tabular}{|c|c|}
\hline DESCRIPTION & LOCUS TAG \\
\hline hypothetical protein & Gaoo68414_113428 \\
\hline hypothetical protein & Gaoo68414_113429 \\
\hline hypothetical protein & Gaoo68414_113430 \\
\hline hypothetical protein & Gaoo68414_113442 \\
\hline hypothetical protein & Gaoo68414_113470 \\
\hline $\begin{array}{r}\text { quaternary ammonium compound-resistance } \\
\text { protein SugE }\end{array}$ & Gaoo68414_113472 \\
\hline hypothetical protein & Gaoo68414_113478 \\
\hline nucleoside $\mathrm{ABC}$ transporter membrane protein & Gaoo68414_113479 \\
\hline simple sugar transport system permease protein & Gaoo68414_113480 \\
\hline basic membrane protein $\mathrm{A}$ & Gaoo68414_113482 \\
\hline Creatinine amidohydrolase & Gaoo68414_113483 \\
\hline Phage integrase family protein & Gaoo68414_113484 \\
\hline hypothetical protein & Gaoo68414_113485 \\
\hline hypothetical protein & Gaoo68414_113487 \\
\hline hypothetical protein & Gaoo68414_113488 \\
\hline Type VI secretion system VasI, EvfG, VC Ao118 & Gaoo68414_113489 \\
\hline hypothetical protein & Gaoo68414_113490 \\
\hline Homeodomain-like domain-containing protein & Gaoo68414_1135 \\
\hline hypothetical protein & Gaoo68414_113505 \\
\hline extracellular solute-binding protein, family 3 & Gaoo68414_11357 \\
\hline hypothetical protein & Gaoo68414_113579 \\
\hline hypothetical protein & Gaoo68414_113580 \\
\hline Protein of unknown function DUF45 & Gaoo68414_1136 \\
\hline PAS domain-containing protein & Gaoo68414_113626 \\
\hline AraC-type DNA-binding protein & Gaoo68414_113641 \\
\hline hypothetical protein & Gaoo68414_113642 \\
\hline hypothetical protein & Gaoo68414_113643 \\
\hline hypothetical protein & Gaoo68414_113644 \\
\hline hypothetical protein & Gaoo68414_113692 \\
\hline hook-length control protein FliK & Gaoo68414_113695 \\
\hline hypothetical protein & Gaoo68414_1137 \\
\hline $\begin{array}{l}\text { LysR family transcriptional regulator, glycine } \\
\text { cleavage system transcriptional activator }\end{array}$ & Gaoo68414_113702 \\
\hline hypothetical protein & Gaoo68414_113709 \\
\hline 2,4-dienoyl-CoA reductase & Gaoo68414_113716 \\
\hline hypothetical protein & Gaoo68414_113717 \\
\hline Phage integrase family protein & Gaoo68414_113718 \\
\hline methionine aminopeptidase, type I (EC 3.4.11.18) & Gaoo68414_113719 \\
\hline
\end{tabular}


Table A.5: continued

\begin{tabular}{|c|c|}
\hline DESCRIPTION & LOCUS TAG \\
\hline FCD domain-containing protein & Gaoo68414_113720 \\
\hline Transposase & Gaoo68414_113721 \\
\hline $\begin{array}{r}\text { His Kinase A (phospho-acceptor) } \\
\text { domain-containing protein }\end{array}$ & Gaoo68414_113722 \\
\hline hypothetical protein & Gaoo68414_113723 \\
\hline transcriptional regulator, AlpA family & Gaoo68414_113724 \\
\hline hypothetical protein & Gaoo68414_113725 \\
\hline hypothetical protein & Gaoo68414_113726 \\
\hline protein of unknown function (DUF4102) & Gaoo68414_113727 \\
\hline mannosyl-3-phosphoglycerate phosphatase & Gaoo68414_113728 \\
\hline glucosyl-3-phosphoglycerate synthase & Gaoo68414_113729 \\
\hline sucrose phosphorylase & Gaoo68414_113730 \\
\hline hypothetical protein & Gaoo68414_113745 \\
\hline MarR family protein & Gaoo68414_113747 \\
\hline hypothetical protein & Gaoo68414_113776 \\
\hline hypothetical protein & Gaoo68414_1138 \\
\hline hypothetical protein & Gaoo68414_11385 \\
\hline hypothetical protein & Gaoo68414_113853 \\
\hline AAA domain-containing protein & Gaoo68414_113862 \\
\hline hypothetical protein & Gaoo68414_113863 \\
\hline hypothetical protein & Gaoo68414_113864 \\
\hline Opacity protein & Gaoo68414_113865 \\
\hline hypothetical protein & Gaoo68414_113867 \\
\hline peptide/nickel transport system permease protein & Gaoo68414_113871 \\
\hline allantoate deiminase & Gaoo68414_113875 \\
\hline HTH-like domain-containing protein & Gaoo68414_113877 \\
\hline PRC-barrel domain-containing protein & Gaoo68414_11388o \\
\hline putative $\mathrm{Mg}^{2+}$ transporter-C $(\mathrm{MgtC})$ family protein & Gaoo68414_113881 \\
\hline PAS domain S-box-containing protein & Gaoo68414_113882 \\
\hline hypothetical protein & Gaoo68414_113883 \\
\hline Signal transduction histidine kinase & Gaoo68414_113884 \\
\hline hypothetical protein & Gaoo68414_113885 \\
\hline hypothetical protein & Gaoo68414_113886 \\
\hline $\mathrm{Cd}^{2+} / \mathrm{Zn}^{2+}$-exporting ATPase & Gaoo68414_113888 \\
\hline hypothetical protein & Gaoo68414_113889 \\
\hline L,D-transpeptidase catalytic domain & Gaoo68414_113890 \\
\hline transcriptional regulator, MerR family & Gaoo68414_113891 \\
\hline NADPH:quinone reductase & Gaoo68414_113897 \\
\hline hypothetical protein & Gaoo68414_1139 \\
\hline
\end{tabular}


Table A.5: continued

\begin{tabular}{rc}
\hline DESCRIPTION & LOCUS TAG \\
\hline $\begin{array}{r}\text { oligopeptide transport system ATP-binding protein } \\
\text { hypothetical protein } \\
\text { hypothetical protein }\end{array}$ & Gaoo68414_113919 \\
Gaoo68414_113935 \\
Glycosyltransferase family 92 & Gaoo68414_113953 \\
capsular polysaccharide export protein & Gaoo68414_113964 \\
hypothetical protein & Gaoo68414_11398 \\
hypothetical protein & Gaoo68414_113999 \\
\hline
\end{tabular}




\section{A.5 XANTHORHODOPSINS IN ROSEOBACTER GROUP BACTERIA}

Figure A.5 visualises the isolation coordinates from Table A.1 on the world map, and xanthorhodopsin-bearing strains are highlighted in red. In addition, the amino acid sequences of roseobacter-family xanthorhodopsins were aligned (via MUSCLE) with those of select other species, spanning both subgroups of the xanthorhodopsins. A ML phylogeny was reconstructed using RAxML's HPC implementation, with the same configuration as given in Section 2.6. Branch support was estimated through 100 bootstrap repetitions.

Figure A.6 presents the final tree, with both xanthorhodopsin subgroups highlighted in different colours. Xanthorhodopsins of roseobacter strains which had not previously been phylogenetically analysed are coloured red. Roseobacter group xanthorhodopsins form one highlysupported cluster in close relation to the second subgroup, which was found to be most abundant in cold and predominantly saline habitats [77].

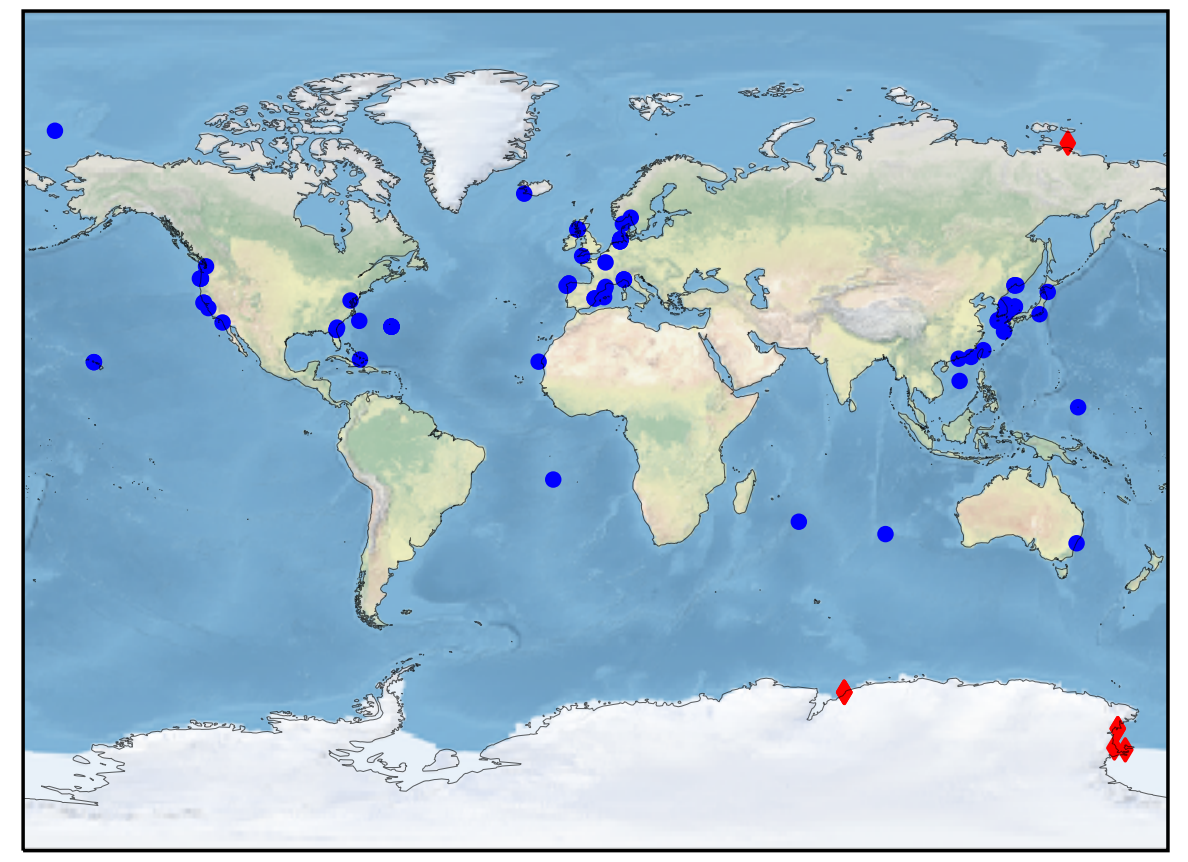

Figure A.5: Isolation coordinates from Table A.1 plotted on the world map. Red diamonds represent strains possessing a xanthorhodopsin. 


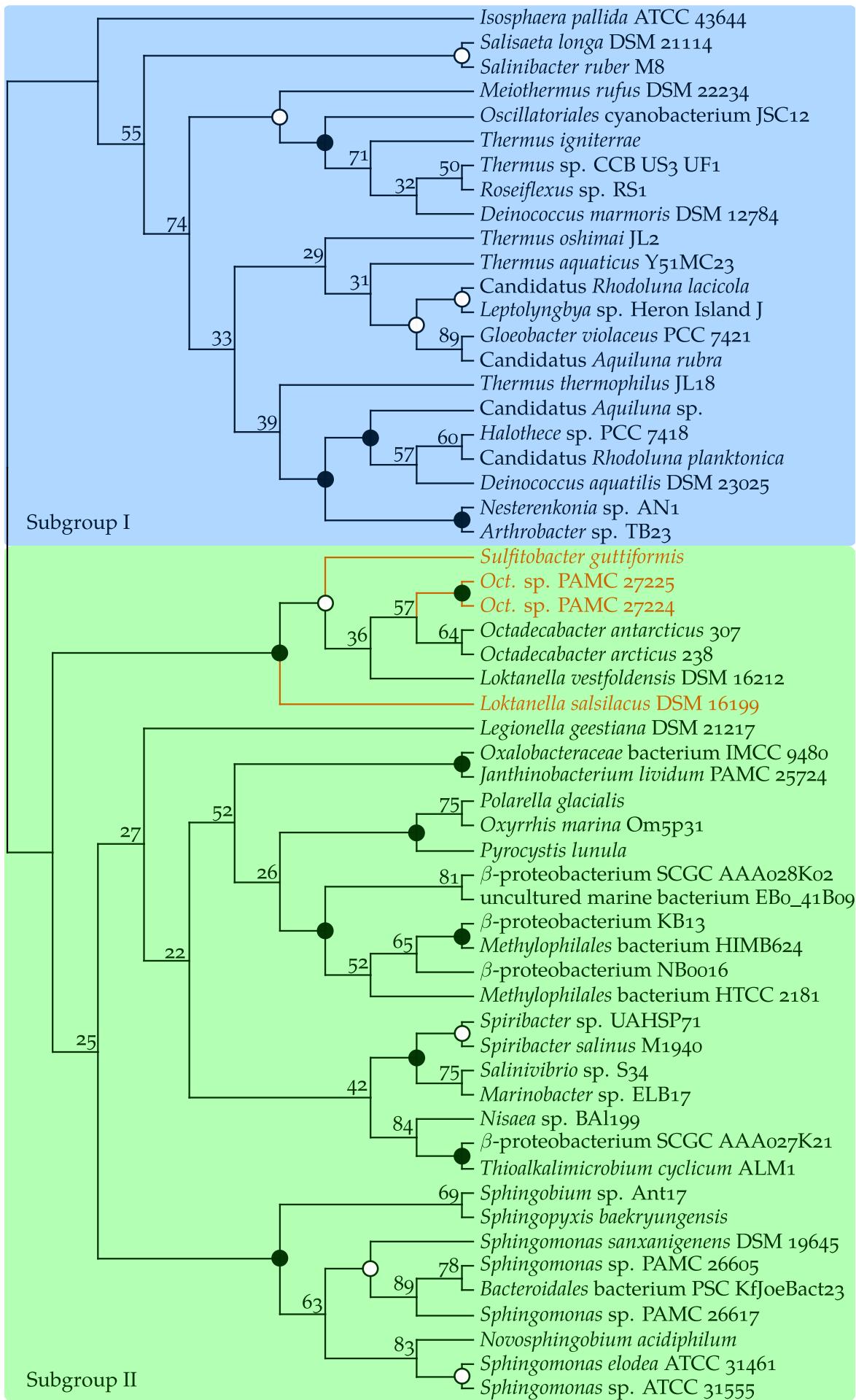

Figure A.6: Phylogenetic placement of Roseobacter group xanthorhodopsins. Subgroups as defined in [77]. Numbers indicate bootstrap support, with hollow and filled circles representing values of 90-99, and 100, respectively. Highlighted roseobacter strains' xanthorhodopsins have not previously been classified. 


\section{A.6 PHYLOGENETIC BIRTH-AND-DEATH MODEL}

Estimated parameter values of the birth-and-death model are visualised for the Octadecabacters in Figure 3.19. Visualisations for the other included strains are given in Figure A.7. Posterior probabilities of COG changes at nodes other than the polar Octadecabacter LCA are provided in Tables A.6 to A.11 (see Figure A.8). They were used to identify changes common to all polar Roseobacter group isolates (Section 3.5).

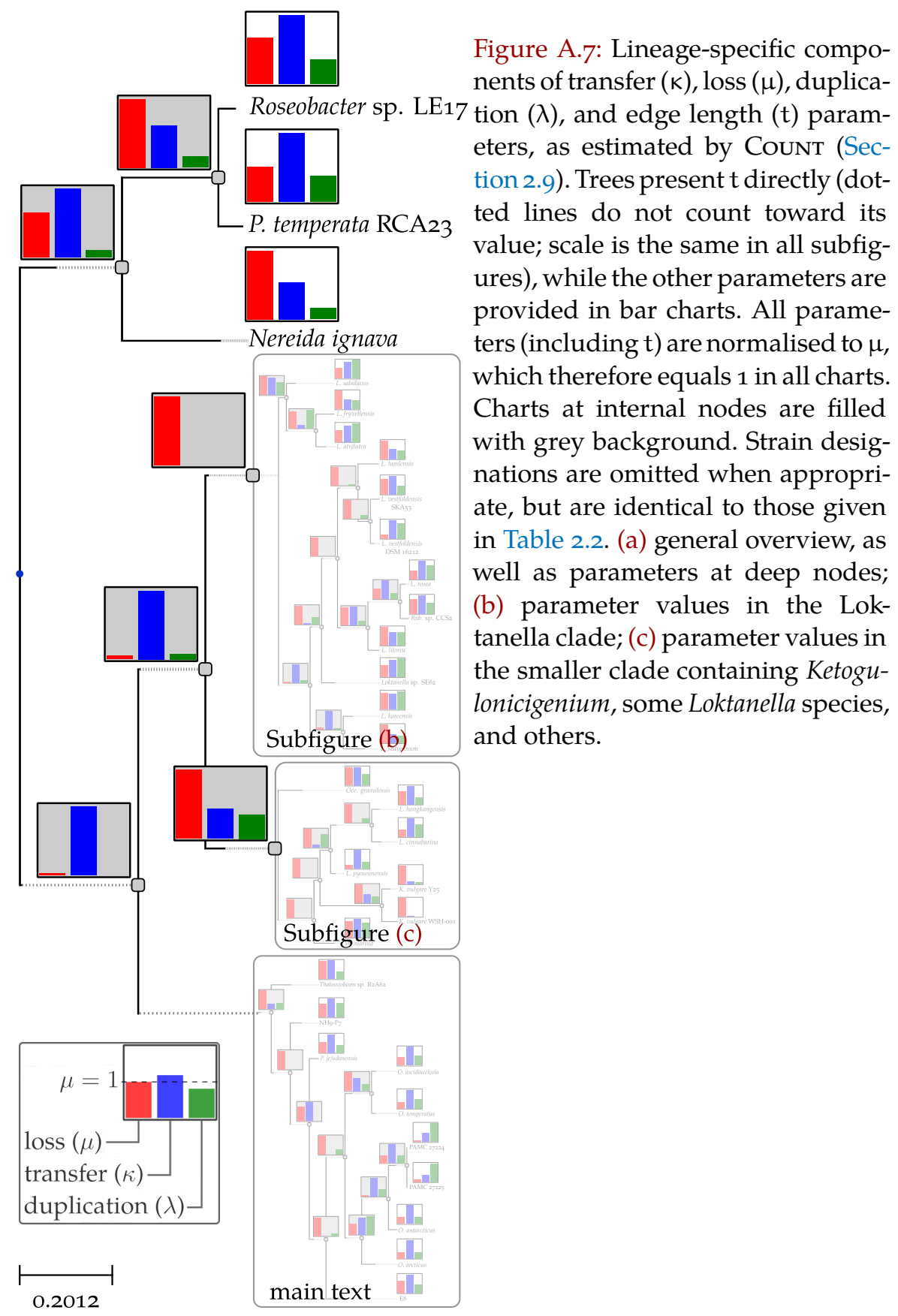

(a) overview 


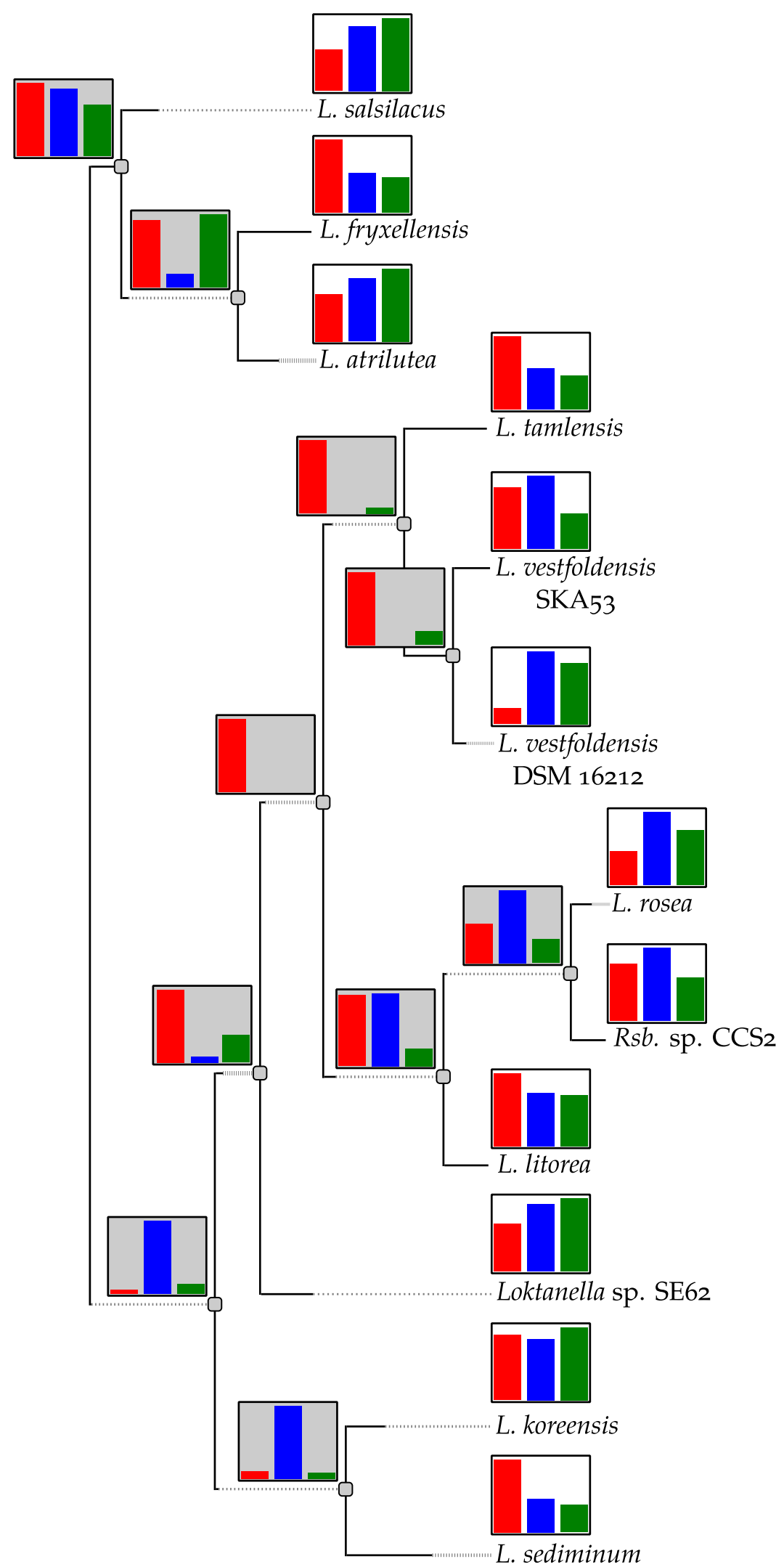

(b) Loktanella genus

Figure A.7: Lineage-specific CounT parameters (continued) 


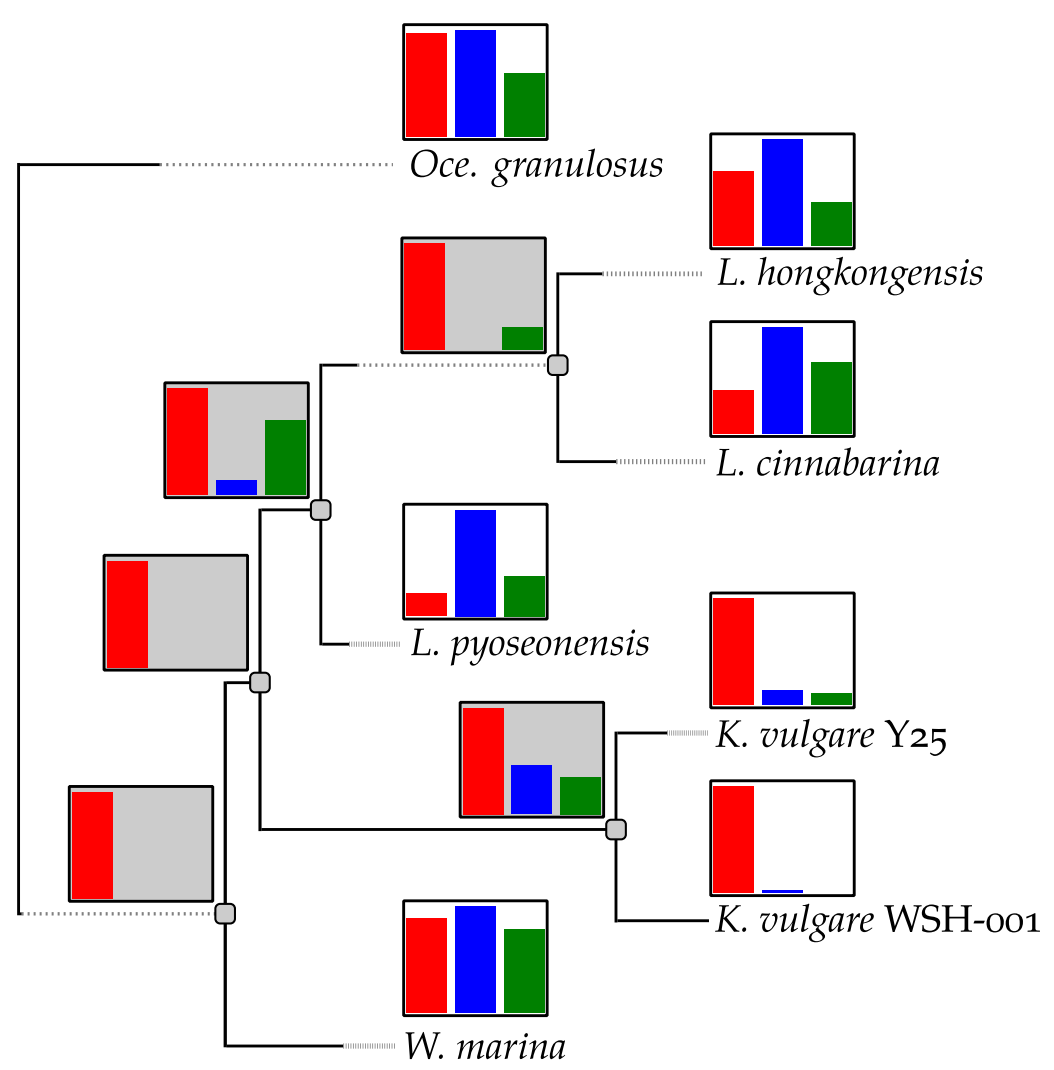

(c) Ketogulonicigenium and others

Figure A.7: Lineage-specific CounT parameters (continued) 


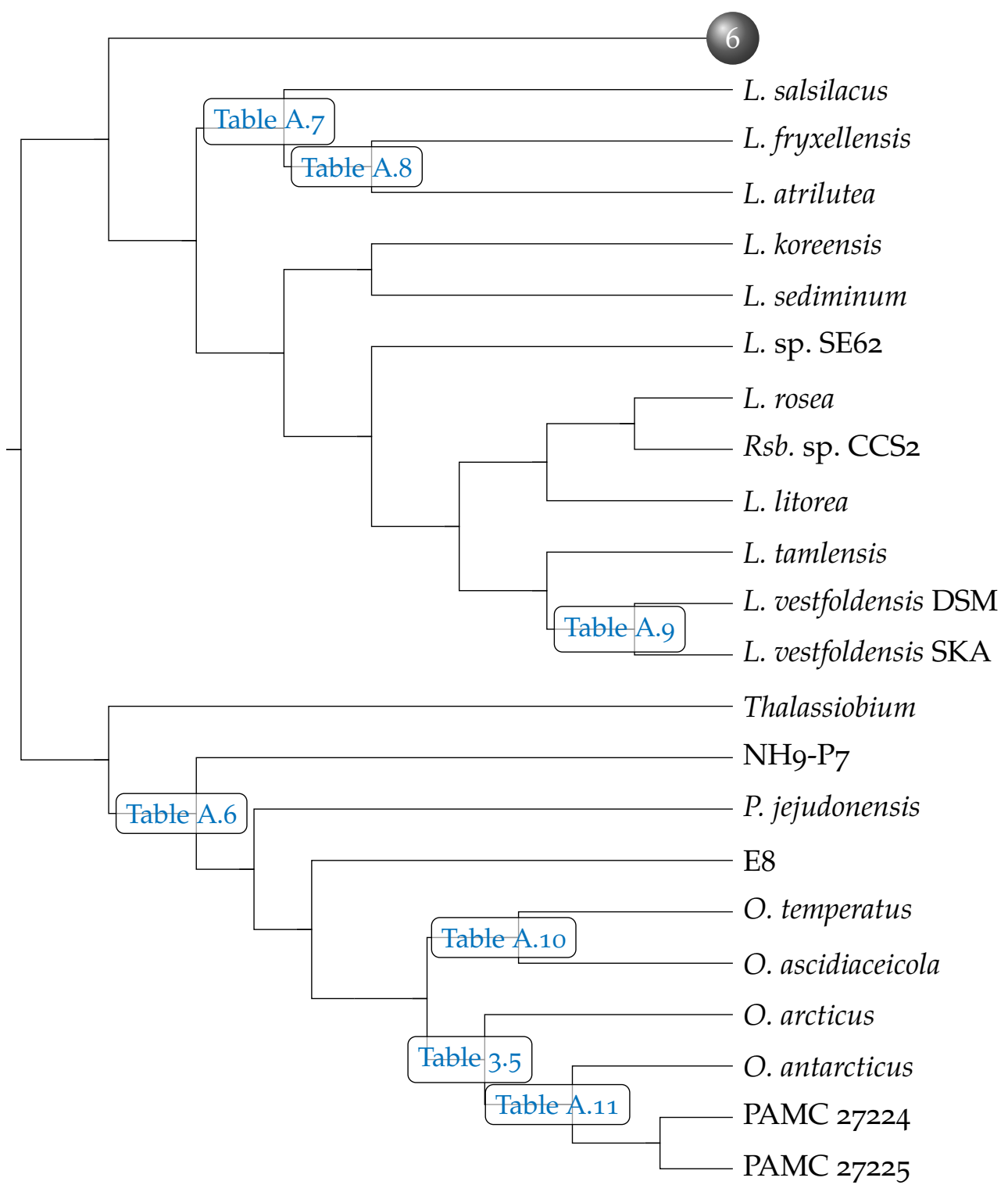

Figure A.8: Tables listing COG changes at specific nodes in Octadecabacter and Loktanella clade phylogeny. The information on gain and loss of specific COGs was used for comparison of changes associated with polar habitats (Section 4.3.4). electronic version - labels at internal nodes of the tree link directly to the respective table 
Table A.6: Changes in COGs at the Octadecabacter clade members' LCA. Only COGs with a probability of change $p>0.5$ at this node are listed. The approximate probability ratios are given in the rightmost column, with green and red indicating gain and loss, respectively. Relevant probabilities are gain $(\mathrm{G})$ : expansion (E) : neutral (N, no change), and loss (L) : reduction (R) : neutral $(\mathrm{N})$. A box indicates the highest probability, and is filled if it surpassed the second-highest by at least $50 \%$. The rightmost column gives the orders of magnitude between the highest and lowest ratio (the latter is always 1). COGs are sorted by category, with the respective category code given on the left. COGs assigned to more than one category are listed multiple times. (back to overview)

\begin{tabular}{|c|c|c|c|c|c|}
\hline \multicolumn{2}{|c|}{ COG ID } & DESCRIPTION & \multicolumn{3}{|c|}{$\mathrm{G}: \mathrm{E}: \mathrm{N} / \mathrm{L}: \mathrm{R}: \mathrm{N}$} \\
\hline $\mathrm{C}$ & 1454 & Alcohol dehydrogenase, class IV & 10 & 1 & 9 \\
\hline G & 3507 & $\beta$-xylosidase & 440 & 1 & 151 \\
\hline $\mathrm{H}$ & 1763 & $\begin{array}{l}\text { Molybdopterin-guanine } \\
\text { dinucleotide biosynthesis } \\
\text { protein }\end{array}$ & 5033 & 1 & 783 \\
\hline \multirow[t]{2}{*}{$\mathrm{P}$} & 2998 & $\begin{array}{l}\text { ABC-type tungstate transport } \\
\text { system, permease component }\end{array}$ & 7705 & 1 & 882 \\
\hline & 4662 & $\begin{array}{l}\text { ABC-type tungstate transport } \\
\text { system, periplasmic component }\end{array}$ & 8562 & 1 & 1287 \\
\hline \multirow[t]{2}{*}{$\mathrm{R}$} & 3565 & $\begin{array}{l}\text { Predicted dioxygenase of } \\
\text { extradiol dioxygenase family }\end{array}$ & 141 & 1 & $4 \quad 10^{3}$ \\
\hline & 4277 & $\begin{array}{l}\text { Predicted DNA-binding protein } \\
\text { with the Helix-hairpin-helix } \\
\text { motif }\end{array}$ & 66 & 1 & $4 \quad 10^{3}$ \\
\hline \multirow[t]{3}{*}{ S } & 4103 & $\begin{array}{l}\text { Uncharacterized conserved } \\
\text { protein, tellurite resistance } \\
\text { protein B (TerB) family }\end{array}$ & 1 & 271 & $20910^{4}$ \\
\hline & 4246 & Uncharacterized protein & 10 & 1 & $310^{5}$ \\
\hline & 4338 & Uncharacterized protein & 6672 & 1 & 1820 \\
\hline $\mathrm{U}$ & 0341 & $\begin{array}{l}\text { Preprotein translocase } \\
\text { subunit SecF }\end{array}$ & 29 & 1 & $1 \quad 10^{4}$ \\
\hline
\end{tabular}

Table A.7: Changes in COGs at the LCA of the two polar Loktanella isolates L. fryxellensis DSM 16213 and L. salsilacus DSM 16199. See description of Table A.6 for detailed information. (back to overview)

\begin{tabular}{cllllll}
\hline COG ID & DESCRIPTION & \multicolumn{3}{c}{ G:E:N/L:R:N } \\
\hline \multirow{2}{*}{0711} & $\begin{array}{l}\text { FoF1-type ATP synthase, } \\
\text { membrane subunit b or b' }\end{array}$ & 1 & 11 & 2 & $10^{3}$ \\
\hline
\end{tabular}


Table A.7: continued (back to overview)

\begin{tabular}{|c|c|c|c|c|c|}
\hline & COG ID & DESCRIPTION & G:E: & N/L:I & $\mathrm{R}: \mathrm{N}$ \\
\hline & 0843 & $\begin{array}{l}\text { Heme/copper-type } \\
\text { cytochrome/quinol oxidase, } \\
\text { subunit } 1\end{array}$ & 1 & 12 & $310^{5}$ \\
\hline & 1145 & Ferredoxin & 91 & 1 & $7 \quad 10^{4}$ \\
\hline & 1182 & $\begin{array}{l}\text { FMN-dependent } \\
\text { NADH-azoreductase }\end{array}$ & 2344 & 1 & 619 \\
\hline & 1301 & $\begin{array}{l}\mathrm{Na}^{+} / \mathrm{H}^{+} \text {-dicarboxylate } \\
\text { symporter }\end{array}$ & 23 & 1 & $210^{5}$ \\
\hline & 1454 & Alcohol dehydrogenase, class IV & 3 & 1 & 3 \\
\hline & 1622 & $\begin{array}{l}\text { Heme/copper-type } \\
\text { cytochrome/quinol oxidase, } \\
\text { subunit } 2\end{array}$ & 1 & 168 & $5^{8} \quad 10^{4}$ \\
\hline & 2326 & $\begin{array}{l}\text { Polyphosphate kinase } 2, \\
\text { PPK2 family }\end{array}$ & 1 & 3181 & 1878 \\
\hline & 2421 & Acetamidase/formamidase & 8118 & 1 & 3960 \\
\hline & 2838 & $\begin{array}{l}\text { Monomeric isocitrate } \\
\text { dehydrogenase }\end{array}$ & 24 & 1 & $210^{3}$ \\
\hline & 2864 & $\begin{array}{l}\text { Cytochrome } b \text { subunit of } \\
\text { formate dehydrogenase }\end{array}$ & 118 & 1 & 30 \\
\hline & 3278 & $\begin{array}{l}\text { Cbb3-type cytochrome oxidase, } \\
\text { subunit } 1\end{array}$ & 1 & 2328 & 1992 \\
\hline \multirow[t]{2}{*}{$\mathrm{D}$} & 1475 & $\begin{array}{l}\text { Chromosome segregation } \\
\text { protein SpooJ, contains ParB-like } \\
\text { nuclease domain }\end{array}$ & 1 & 40 & $4 \quad 10^{4}$ \\
\hline & 3773 & $\begin{array}{l}\text { Cell wall hydrolase CwlJ, } \\
\text { involved in spore germination }\end{array}$ & 1 & 16 & $210^{5}$ \\
\hline \multirow{7}{*}{$\mathrm{E}$} & 0019 & Diaminopimelate decarboxylase & 1 & 24 & $9 \quad 10^{4}$ \\
\hline & 0069 & Glutamate synthase domain 2 & 1 & 1886 & 582 \\
\hline & 0405 & Gamma-glutamyltranspeptidase & 1 & 29 & $1410^{3}$ \\
\hline & 0686 & Alanine dehydrogenase & 1 & 428 & 48 \\
\hline & O754 & $\begin{array}{l}\text { Glutathionylspermidine } \\
\text { synthase }\end{array}$ & 20 & 1 & $210^{4}$ \\
\hline & 1003 & $\begin{array}{l}\text { Glycine cleavage system } \\
\text { protein P (pyridoxal-binding), } \\
\text { C-terminal domain }\end{array}$ & 5454 & 1 & 1034 \\
\hline & 1124 & $\begin{array}{l}\text { ABC-type } \\
\text { dipeptide/oligopeptide/nickel } \\
\text { transport system, ATPase } \\
\text { component }\end{array}$ & 19 & 1 & $1110^{3}$ \\
\hline
\end{tabular}


Table A.7: continued (back to overview)

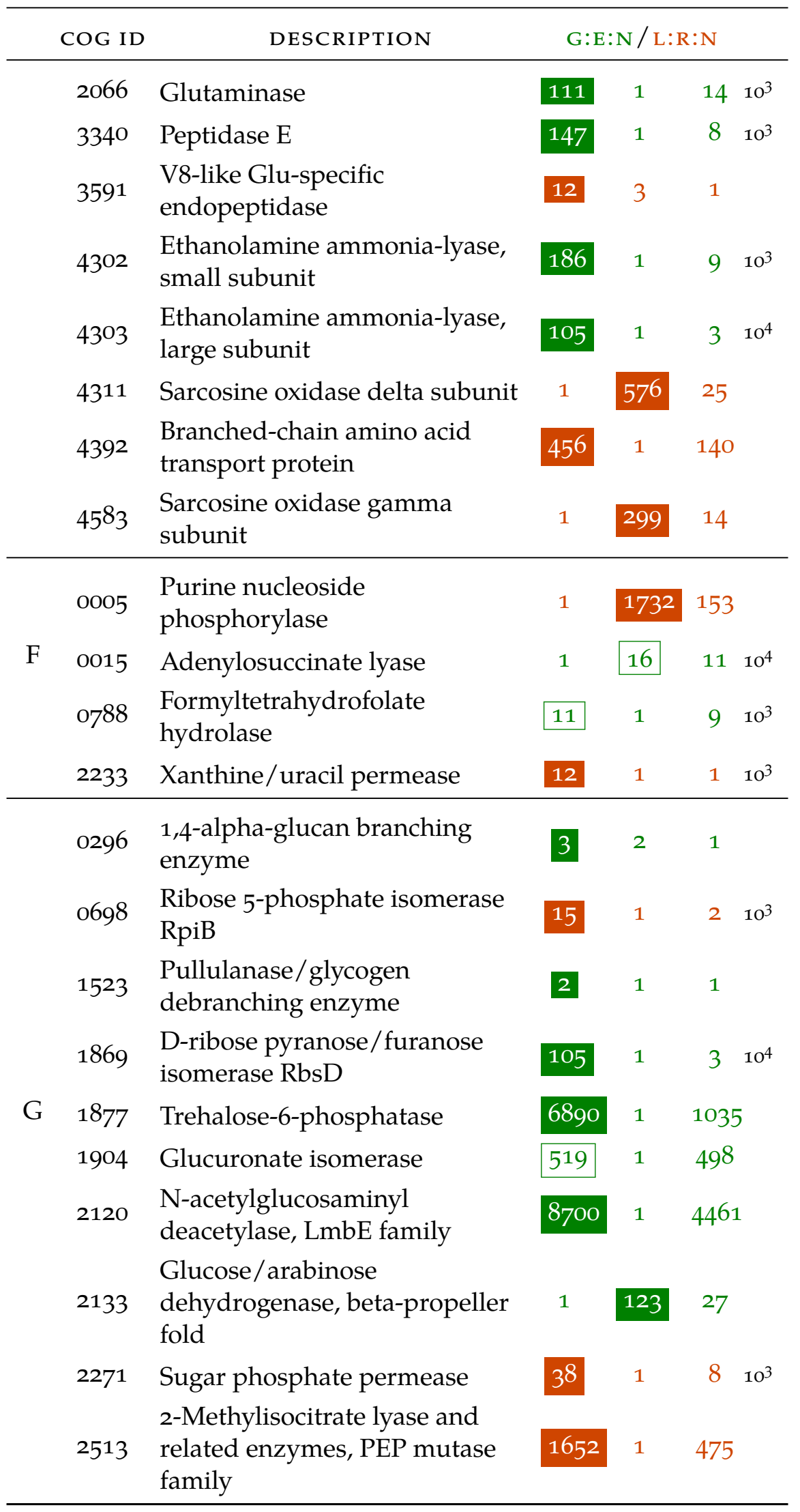


Table A.7: continued (back to overview)

\begin{tabular}{|c|c|c|c|c|c|}
\hline & COG ID & DESCRIPTION & G:E: & N/L:F & $\mathrm{R}: \mathrm{N}$ \\
\hline & 2861 & $\begin{array}{l}\text { Uncharacterized conserved } \\
\text { protein YibQ, putative } \\
\text { polysaccharide deacetylase } 2 \\
\text { family }\end{array}$ & 28 & 1 & $5 \quad 10^{3}$ \\
\hline & 2943 & Membrane glycosyltransferase & 493 & 1 & 433 \\
\hline & 3280 & Maltooligosyltrehalose synthase & 3345 & 1 & 829 \\
\hline & 3717 & 5-keto 4-deoxyuronate isomerase & 530 & 1 & 177 \\
\hline & 4993 & Glucose dehydrogenase & 15 & 1 & $4 \quad 10^{3}$ \\
\hline \multirow{4}{*}{$\mathrm{H}$} & 0161 & $\begin{array}{l}\text { Adenosylmethionine-8-amino-7- } \\
\text { oxononanoate } \\
\text { aminotransferase }\end{array}$ & 1 & 4134 & 787 \\
\hline & 1335 & Nicotinamidase-related amidase & 1 & 90 & $3210^{4}$ \\
\hline & 2141 & $\begin{array}{l}\text { Flavin-dependent } \\
\text { oxidoreductase, luciferase family } \\
\text { (includes alkanesulfonate } \\
\text { monooxygenase SsuD and } \\
\text { methylene } \\
\text { tetrahydromethanopterin } \\
\text { reductase) }\end{array}$ & 1 & 56 & $51 \quad 10^{4}$ \\
\hline & 5598 & $\begin{array}{l}\text { Trimethylamine:corrinoid } \\
\text { methyltransferase }\end{array}$ & 1 & 117 & 13 \\
\hline \multirow{8}{*}{$\mathrm{I}$} & 0236 & Acyl carrier protein & 1 & 26 & $1910^{3}$ \\
\hline & 0657 & Acetyl esterase/lipase & 1 & 62 & $1410^{4}$ \\
\hline & 0688 & $\begin{array}{l}\text { Phosphatidylserine } \\
\text { decarboxylase }\end{array}$ & 10 & 1 & $210^{3}$ \\
\hline & 0818 & Diacylglycerol kinase & 6504 & 1 & 777 \\
\hline & 1183 & Phosphatidylserine synthase & 1 & 163 & $1310^{4}$ \\
\hline & 1597 & $\begin{array}{l}\text { Diacylglycerol kinase family } \\
\text { enzyme }\end{array}$ & 1200 & 1 & 626 \\
\hline & 2267 & $\begin{array}{l}\text { Lysophospholipase, alpha-beta } \\
\text { hydrolase superfamily }\end{array}$ & 1 & 177 & $3910^{3}$ \\
\hline & 2854 & $\begin{array}{l}\text { ABC-type transporter Mla } \\
\text { maintaining outer membrane } \\
\text { lipid asymmetry, periplasmic } \\
\text { MlaC component }\end{array}$ & 23 & 1 & $210^{3}$ \\
\hline $\mathrm{J}$ & 0590 & $\begin{array}{l}\text { tRNA(Arg) A34 adenosine } \\
\text { deaminase TadA }\end{array}$ & 1 & 309 & $3210^{4}$ \\
\hline
\end{tabular}


Table A.7: continued (back to overview)

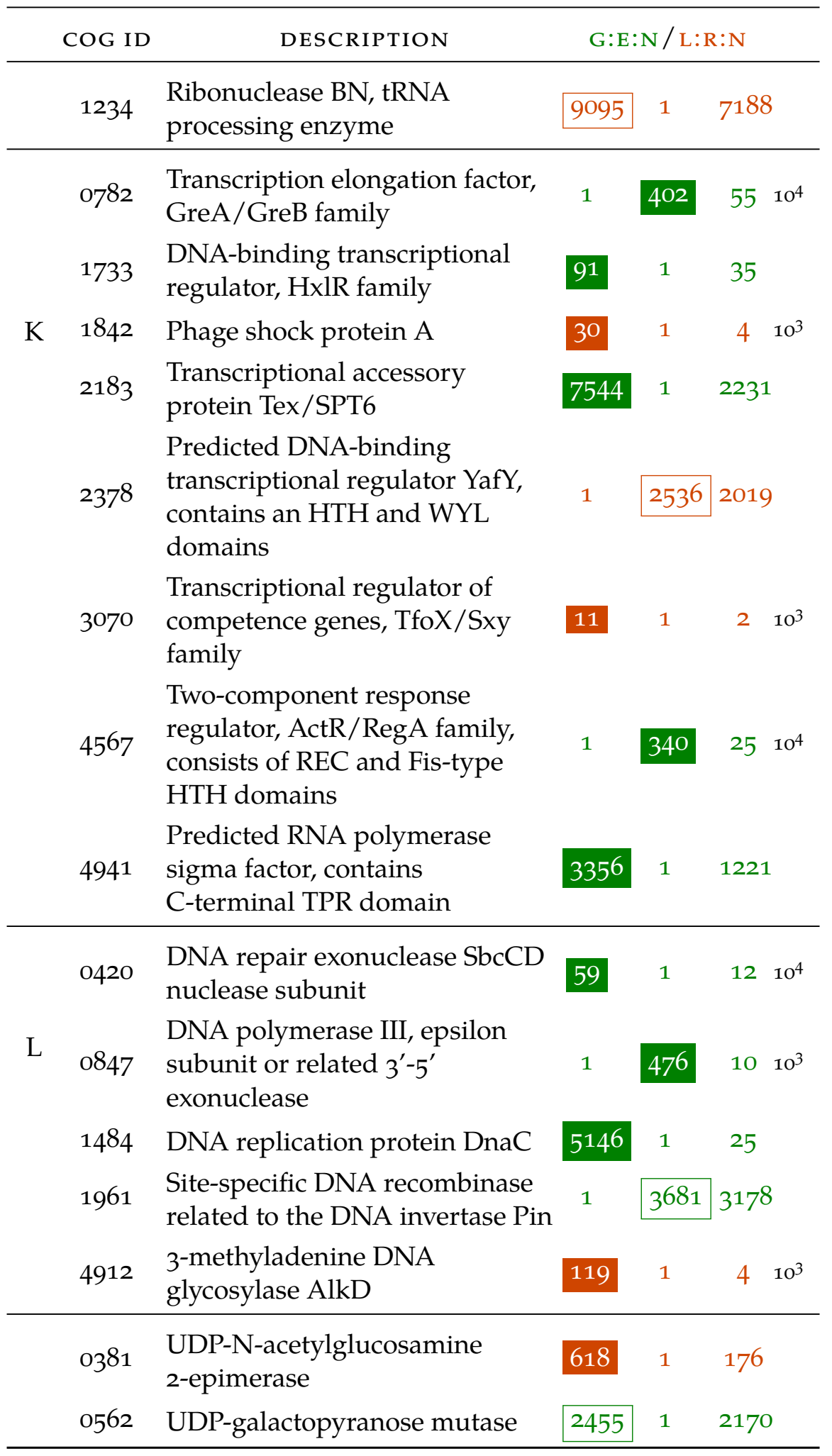


Table A.7: continued (back to overview)

\begin{tabular}{|c|c|c|c|c|c|}
\hline & COG ID & DESCRIPTION & G:E: & N/L:R & $\mathrm{R}: \mathrm{N}$ \\
\hline & 0767 & $\begin{array}{l}\text { ABC-type transporter Mla } \\
\text { maintaining outer membrane } \\
\text { lipid asymmetry, permease } \\
\text { component MlaE }\end{array}$ & 1 & 75 & 31 \\
\hline & 1127 & $\begin{array}{l}\text { ABC-type transporter Mla } \\
\text { maintaining outer membrane } \\
\text { lipid asymmetry, ATPase } \\
\text { component MlaF }\end{array}$ & 1 & 75 & 31 \\
\hline & 1210 & $\begin{array}{l}\text { UTP-glucose-1-phosphate } \\
\text { uridylyltransferase }\end{array}$ & 1 & 36 & $3310^{4}$ \\
\hline & 1463 & $\begin{array}{l}\text { ABC-type transporter Mla } \\
\text { maintaining outer membrane } \\
\text { lipid asymmetry, periplasmic } \\
\text { component MlaD }\end{array}$ & 1 & 75 & 31 \\
\hline & 1538 & Outer membrane protein TolC & 1 & 143 & $5310^{4}$ \\
\hline & 2825 & $\begin{array}{l}\text { Periplasmic chaperone for outer } \\
\text { membrane proteins, Skp family }\end{array}$ & 1099 & 1 & 562 \\
\hline & 2853 & $\begin{array}{l}\text { ABC-type transporter Mla } \\
\text { maintaining outer membrane } \\
\text { lipid asymmetry, lipoprotein } \\
\text { component MlaA }\end{array}$ & 22 & 1 & $310^{3}$ \\
\hline & 2943 & Membrane glycosyltransferase & 493 & 1 & 433 \\
\hline & 3307 & O-antigen ligase & 52 & 1 & $3210^{3}$ \\
\hline & 3757 & $\begin{array}{l}\text { Lyzozyme M1 } \\
\text { (1,4-beta-N-acetylmuramidase), } \\
\text { GH25 family }\end{array}$ & 28 & 1 & $4 \quad 10^{3}$ \\
\hline & 3773 & $\begin{array}{l}\text { Cell wall hydrolase CwlJ, } \\
\text { involved in spore germination }\end{array}$ & 1 & 164 & $1710^{4}$ \\
\hline $\mathrm{N}$ & 1352 & $\begin{array}{l}\text { Methylase of chemotaxis } \\
\text { methyl-accepting proteins }\end{array}$ & 5 & 1 & 1 \\
\hline \multirow{5}{*}{$\mathrm{O}$} & 0501 & $\begin{array}{l}\text { Zn-dependent protease with } \\
\text { chaperone function }\end{array}$ & 13 & 1 & 13 \\
\hline & 0846 & $\begin{array}{l}\text { NAD-dependent protein } \\
\text { deacetylase, SIR } 2 \text { family }\end{array}$ & 17 & 1 & $210^{3}$ \\
\hline & 1404 & Serine protease, subtilisin family & 24 & 1 & $610^{3}$ \\
\hline & 1858 & Cytochrome c peroxidase & 1 & 794 & 94 \\
\hline & 2135 & $\begin{array}{l}\text { Putative SOS } \\
\text { response-associated peptidase } \\
\text { YedK }\end{array}$ & 1 & 3234 & 321 \\
\hline
\end{tabular}


Table A.7: continued (back to overview)

\begin{tabular}{|c|c|c|c|c|c|c|}
\hline \multirow{2}{*}{\multicolumn{2}{|c|}{$\begin{array}{l}\text { COG ID } \\
2825\end{array}$}} & \multirow{2}{*}{$\begin{array}{l}\text { DESCRIPTION } \\
\text { mic chaperone for outer } \\
\text { ane proteins, Skp family }\end{array}$} & \multicolumn{4}{|c|}{$\mathrm{G}: \mathrm{E}: \mathrm{N} / \mathrm{L}: \mathrm{R}: \mathrm{N}$} \\
\hline & & & 1099 & 1 & 562 & \\
\hline & 3187 & Heat shock protein HslJ & 658 & 1 & 288 & \\
\hline & 3381 & $\begin{array}{l}\text { Cytoplasmic chaperone TorD } \\
\text { involved in molybdoenzyme } \\
\text { TorA maturation }\end{array}$ & 677 & 1 & 348 & \\
\hline & 4960 & $\begin{array}{l}\text { Flp pilus assembly protein, } \\
\text { protease CpaA }\end{array}$ & 995 & 1 & 201 & \\
\hline \multirow{13}{*}{$\mathrm{P}$} & 0053 & $\begin{array}{l}\text { Divalent metal cation } \\
(\mathrm{Fe} / \mathrm{Co} / \mathrm{Zn} / \mathrm{Cd}) \text { transporter }\end{array}$ & 25 & 1 & 19 & \\
\hline & 0306 & Phosphate/sulfate permease & 144 & 1 & 8 & $10^{2}$ \\
\hline & 0569 & $\begin{array}{l}\text { Trk } \mathrm{K}^{+} \text {transport system, } \\
\text { NAD-binding component }\end{array}$ & 1 & 293 & 43 & $10^{4}$ \\
\hline & 0598 & $\begin{array}{l}\mathrm{Mg}^{2+} \text { and } \mathrm{Co}^{2+} \text { transporter } \\
\text { CorA }\end{array}$ & 27 & 1 & 25 & \\
\hline & $\mathrm{O} 748$ & $\begin{array}{l}\text { Putative heme iron utilization } \\
\text { protein }\end{array}$ & 16 & 1 & 3 & $10^{3}$ \\
\hline & 0753 & Catalase & 206 & 1 & 42 & $10^{3}$ \\
\hline & 1122 & $\begin{array}{l}\text { Energy-coupling factor } \\
\text { transporter ATP-binding protein } \\
\text { EcfA2 }\end{array}$ & 38 & 1 & 10 & $10^{3}$ \\
\hline & 1124 & $\begin{array}{l}\text { ABC-type } \\
\text { dipeptide/oligopeptide/nickel } \\
\text { transport system, ATPase } \\
\text { component }\end{array}$ & 19 & 1 & 11 & $10^{3}$ \\
\hline & 1230 & $\begin{array}{l}\mathrm{Co} / \mathrm{Zn} / \mathrm{Cd} \text { efflux system } \\
\text { component }\end{array}$ & 7 & 1 & 8 & \\
\hline & 2608 & Copper chaperone CopZ & 2556 & 1 & 775 & \\
\hline & 3119 & $\begin{array}{l}\text { Arylsulfatase A or related } \\
\text { enzyme }\end{array}$ & 1 & 1 & 1 & \\
\hline & $315^{8}$ & $\mathrm{~K}^{+}$transporter & 186 & 1 & 8 & $10^{4}$ \\
\hline & 3685 & $\begin{array}{l}\text { Ferritin-like metal-binding } \\
\text { protein YciE }\end{array}$ & 814 & 1 & 366 & \\
\hline \multirow{3}{*}{ Q } & 0236 & Acyl carrier protein & 1 & 26 & 19 & \\
\hline & 3485 & $\begin{array}{l}\text { Protocatechuate 3,4-dioxygenase } \\
\text { beta subunit }\end{array}$ & 7 & 1 & 2 & \\
\hline & 3509 & $\begin{array}{l}\text { Poly(3-hydroxybutyrate) } \\
\text { depolymerase }\end{array}$ & 14 & 1 & 9 & \\
\hline
\end{tabular}


Table A.7: continued (back to overview)

\begin{tabular}{|c|c|c|c|c|c|}
\hline & COG ID & DESCRIPTION & G:E: & N/L:R & $\mathrm{R}: \mathrm{N}$ \\
\hline & 5285 & $\begin{array}{l}\text { Ectoine hydroxylase-related } \\
\text { dioxygenase, phytanoyl-CoA } \\
\text { dioxygenase (PhyH) family }\end{array}$ & 353 & 1 & 62 \\
\hline \multirow{14}{*}{$\mathrm{R}$} & 0121 & $\begin{array}{l}\text { Predicted glutamine } \\
\text { amidotransferase }\end{array}$ & 50 & 1 & $910^{3}$ \\
\hline & 0388 & Predicted amidohydrolase & 1 & 345 & $72 \quad 10^{3}$ \\
\hline & 0599 & $\begin{array}{l}\text { Uncharacterized conserved } \\
\text { protein YurZ, alkylhydroperoxi- } \\
\text { dase/carboxymuconolactone } \\
\text { decarboxylase family }\end{array}$ & 1 & 194 & $5410^{3}$ \\
\hline & 1122 & $\begin{array}{l}\text { Energy-coupling factor } \\
\text { transporter ATP-binding protein } \\
\text { EcfA2 }\end{array}$ & 38 & 1 & $1010^{3}$ \\
\hline & 1335 & Nicotinamidase-related amidase & 1 & 89 & $3210^{4}$ \\
\hline & 1597 & $\begin{array}{l}\text { Diacylglycerol kinase family } \\
\text { enzyme }\end{array}$ & 1200 & 1 & 626 \\
\hline & 1611 & $\begin{array}{l}\text { Predicted Rossmann fold } \\
\text { nucleotide-binding protein }\end{array}$ & 1 & 9724 & 729 \\
\hline & 1741 & $\begin{array}{l}\text { Redox-sensitive bicupin YhaK, } \\
\text { pirin superfamily }\end{array}$ & 1 & 12 & 6 \\
\hline & 1917 & $\begin{array}{l}\text { Cupin domain protein related to } \\
\text { quercetin dioxygenase }\end{array}$ & 1 & 5060 & 1964 \\
\hline & 2124 & $\begin{array}{l}\text { Flavin-dependent } \\
\text { oxidoreductase, luciferase family } \\
\text { (includes alkanesulfonate } \\
\text { monooxygenase SsuD and } \\
\text { methylene } \\
\text { tetrahydromethanopterin } \\
\text { reductase) }\end{array}$ & 1 & 56 & $51 \quad 10^{4}$ \\
\hline & 2261 & $\begin{array}{l}\text { Uncharacterized membrane } \\
\text { protein YeaQ/YmgE, } \\
\text { transglycosylase-associated } \\
\text { protein family }\end{array}$ & 10 & 1 & $4 \quad 10^{3}$ \\
\hline & 2350 & $\begin{array}{l}\text { Uncharacterized conserved } \\
\text { protein YciI, contains a putative } \\
\text { active-site phosphohistidine }\end{array}$ & 4879 & 1 & 130 \\
\hline & 3128 & $\begin{array}{l}\text { Predicted 2-oxoglutarate- and } \\
\text { Fe(II)-dependent dioxygenase } \\
\text { YbiX }\end{array}$ & 78 & 1 & $2610^{3}$ \\
\hline & 3360 & Flavin-binding protein dodecin & 8640 & 1 & 1541 \\
\hline
\end{tabular}


Table A.7: continued (back to overview)

\begin{tabular}{|c|c|c|c|c|}
\hline COG ID & DESCRIPTION & G:E & $\mathrm{N} / \mathrm{L}:$ & $: N$ \\
\hline 3800 & $\begin{array}{l}\text { Predicted transcriptional } \\
\text { regulator }\end{array}$ & 7 & 22 & 1 \\
\hline 4132 & $\begin{array}{l}\text { ABC-type uncharacterized } \\
\text { transport system, permease } \\
\text { component }\end{array}$ & 105 & 1 & $310^{4}$ \\
\hline 4589 & $\begin{array}{l}\text { Predicted CDP-diglyceride } \\
\text { synthetase/phosphatidate } \\
\text { cytidylyltransferase }\end{array}$ & 32 & 1 & $210^{3}$ \\
\hline 4666 & $\begin{array}{l}\text { TRAP-type uncharacterized } \\
\text { transport system, fused } \\
\text { permease components }\end{array}$ & 1 & 21 & 20 \\
\hline 4783 & $\begin{array}{l}\text { Putative } \mathrm{Zn} \text {-dependent protease, } \\
\text { contains TPR repeats }\end{array}$ & 1 & 50 & 41 \\
\hline 5496 & Predicted thioesterase & 105 & 1 & $310^{4}$ \\
\hline 0586 & $\begin{array}{l}\text { Uncharacterized membrane } \\
\text { protein DedA, } \\
\text { SNARE-associated domain }\end{array}$ & 5 & 9 & 1 \\
\hline 2121 & $\begin{array}{l}\text { Uncharacterized conserved } \\
\text { protein, lysophospholipid } \\
\text { acyltransferase (LPLAT) } \\
\text { superfamily }\end{array}$ & 44 & 1 & $4 \quad 10^{3}$ \\
\hline 2308 & $\begin{array}{l}\text { Uncharacterized conserved } \\
\text { protein, circularly permuted } \\
\text { ATPgrasp superfamily }\end{array}$ & 1 & 4 & 1 \\
\hline 2860 & $\begin{array}{l}\text { Uncharacterized membrane } \\
\text { protein YeiH }\end{array}$ & 23 & 1 & $5 \quad 10^{3}$ \\
\hline 2898 & $\begin{array}{l}\text { Lysylphosphatidylglycerol } \\
\text { synthetase, C-terminal domain, } \\
\text { DUF2156 family }\end{array}$ & 1 & 20 & 11 \\
\hline 2979 & $\begin{array}{l}\text { Uncharacterized membrane } \\
\text { protein YebE, DUF } 533 \text { family }\end{array}$ & 11 & 1 & 3 \\
\hline 3329 & $\begin{array}{l}\text { Uncharacterized conserved } \\
\text { protein }\end{array}$ & 1 & 1 & 2 \\
\hline 3490 & Uncharacterized protein & 476 & 1 & 313 \\
\hline 3514 & $\begin{array}{l}\text { Uncharacterized conserved } \\
\text { protein, DUF } 4415 \text { family }\end{array}$ & 1099 & 1 & 302 \\
\hline 3544 & $\begin{array}{l}\text { Uncharacterized conserved } \\
\text { protein, } \mathrm{DUF}_{305} \text { family }\end{array}$ & 20 & 2 & 1 \\
\hline 3603 & Uncharacterized protein & 66 & 1 & $60 \quad 10^{3}$ \\
\hline
\end{tabular}


Table A.7: continued (back to overview)

\begin{tabular}{|c|c|c|c|c|c|c|}
\hline & COG ID & DESCRIPTION & G:E: & N/L:F & $\mathrm{R}: \mathrm{N}$ & \\
\hline & 3673 & $\begin{array}{l}\text { Uncharacterized protein, } \\
\text { PA2063/DUF2235 family }\end{array}$ & 1 & 48 & 33 & \\
\hline & 3739 & $\begin{array}{l}\text { Uncharacterized membrane } \\
\text { protein YoaT, DUF817 family }\end{array}$ & 33 & 1 & 5 & $10^{3}$ \\
\hline & 3795 & $\begin{array}{l}\text { Uncharacterized conserved } \\
\text { protein }\end{array}$ & 5025 & 1 & 2271 & \\
\hline & 3921 & $\begin{array}{l}\text { Uncharacterized conserved } \\
\text { protein }\end{array}$ & 1 & 1547 & 743 & \\
\hline & 4196 & $\begin{array}{l}\text { Uncharacterized conserved } \\
\text { protein, DUF } 2126 \text { family }\end{array}$ & 147 & 1 & 25 & $10^{3}$ \\
\hline & 4275 & Uncharacterized protein & 50 & 1 & 9 & $10^{3}$ \\
\hline & 4286 & $\begin{array}{l}\text { Uncharacterized protein, } \\
\text { UPFo16o family }\end{array}$ & 930 & 1 & $55^{2}$ & \\
\hline & 4298 & Uncharacterized protein & 30 & 1 & 4 & $10^{3}$ \\
\hline & 4307 & Uncharacterized protein & 228 & 1 & 17 & $10^{3}$ \\
\hline & 4325 & $\begin{array}{l}\text { Uncharacterized membrane } \\
\text { protein }\end{array}$ & 1 & 5 & 5 & \\
\hline & 4405 & Uncharacterized protein YhfF & 1 & 297 & 135 & \\
\hline & 4625 & $\begin{array}{l}\text { Uncharacterized conserved } \\
\text { protein, contains a C-terminal } \\
\text { beta-barrel porin domain }\end{array}$ & 21 & 1 & 5 & $10^{3}$ \\
\hline & 4717 & Uncharacterized protein YhaN & 389 & 1 & 49 & $10^{3}$ \\
\hline & 4991 & $\begin{array}{l}\text { Uncharacterized conserved } \\
\text { protein YraI }\end{array}$ & 136 & 1 & 37 & \\
\hline & 5463 & $\begin{array}{l}\text { Uncharacterized conserved } \\
\text { protein YgiB, involved in } \\
\text { bioifilm formation, } \\
\text { UPFo441/DUF119o family }\end{array}$ & 111 & 1 & 8 & $10^{3}$ \\
\hline & 5481 & Uncharacterized protein & 10 & 1 & 1 & $10^{3}$ \\
\hline & 5579 & $\begin{array}{l}\text { Uncharacterized protein, } \\
\text { DUF1810 family }\end{array}$ & 1200 & 1 & 626 & \\
\hline \multirow{3}{*}{$\mathrm{T}$} & 1352 & $\begin{array}{l}\text { Methylase of chemotaxis } \\
\text { methyl-accepting proteins }\end{array}$ & 5 & 1 & 1 & \\
\hline & 1842 & Phage shock protein A & 30 & 1 & 4 & $10^{3}$ \\
\hline & 3806 & $\begin{array}{l}\text { Anti-sigma factor ChrR, cupin } \\
\text { superfamily }\end{array}$ & 1 & 38 & 2 & \\
\hline
\end{tabular}


Table A.7: continued (back to overview)

\begin{tabular}{|c|c|c|c|c|c|}
\hline \multicolumn{2}{|c|}{ COG ID } & DESCRIPTION & \multicolumn{3}{|c|}{ G:E:N/L:R:N } \\
\hline & 4567 & $\begin{array}{l}\text { Two-component response } \\
\text { regulator, ActR/RegA family, } \\
\text { consists of REC and Fis-type } \\
\text { HTH domains }\end{array}$ & 1 & 340 & $25 \quad 10^{4}$ \\
\hline & 4960 & $\begin{array}{l}\text { Flp pilus assembly protein, } \\
\text { protease CpaA }\end{array}$ & 995 & 1 & 201 \\
\hline \multirow[t]{2}{*}{ V } & 1764 & $\begin{array}{l}\text { Organic hydroperoxide } \\
\text { reductase OsmC/OhrA }\end{array}$ & 3728 & 1 & 1584 \\
\hline & 4845 & $\begin{array}{l}\text { Chloramphenicol } \\
\text { O-acetyltransferase }\end{array}$ & 23 & 1 & $4 \quad 10^{3}$ \\
\hline \multirow{3}{*}{$X$} & 3547 & Transposase & 732 & 1 & 241 \\
\hline & 4584 & Transposase & 5756 & 1 & 3472 \\
\hline & 5534 & $\begin{array}{l}\text { Plasmid replication initiator } \\
\text { protein }\end{array}$ & 1432 & 1 & 448 \\
\hline
\end{tabular}

Table A.8: Changes in COGs at the LCA of the polar Loktanella isolate L. fryxellensis DSM 16213 and the temperate isolate L. atrilutea DSM 29326. See description of Table A.6 for detailed information. (back to overview)

\begin{tabular}{|c|c|c|c|c|c|}
\hline \multicolumn{2}{|c|}{ COG ID } & \multirow{2}{*}{$\frac{\text { DESCRIPTION }}{\text { Endo-1,4-beta-D-glucanase } \mathrm{Y}}$} & \multicolumn{3}{|c|}{$\mathrm{G}: \mathrm{E}: \mathrm{N} / \mathrm{L}: \mathrm{R}: \mathrm{N}$} \\
\hline G & 3405 & & 2768 & 1 & 2471 \\
\hline $\mathrm{J}$ & 4123 & $\begin{array}{l}\text { tRNA1(Val) A37 N6-methylase } \\
\text { TrmN6 }\end{array}$ & 65 & 1 & $21 \quad 10^{4}$ \\
\hline \multirow[t]{2}{*}{$\mathrm{P}$} & 1910 & $\begin{array}{l}\text { Periplasmic molybdate-binding } \\
\text { protein/domain }\end{array}$ & 2820 & 1 & 1939 \\
\hline & 2216 & $\begin{array}{l}\text { High-affinity } \mathrm{K}^{+} \text {transport } \\
\text { system, ATPase chain B }\end{array}$ & 233 & 1 & 82 \\
\hline \multirow{4}{*}{$\mathrm{R}$} & 2144 & $\begin{array}{l}\text { Selenophosphate } \\
\text { synthetase-related protein }\end{array}$ & 2654 & 1 & 1845 \\
\hline & 2516 & $\begin{array}{l}\text { Biotin synthase-related protein, } \\
\text { radical SAM superfamily }\end{array}$ & 2654 & 1 & 1845 \\
\hline & 3864 & $\begin{array}{l}\text { Predicted metal-dependent } \\
\text { peptidase }\end{array}$ & 3111 & 1 & 2120 \\
\hline & 4671 & Predicted glycosyl transferase & 216 & 1 & 85 \\
\hline$S$ & 3506 & $\begin{array}{l}\text { Regulation of enolase protein } 1 \\
\text { (function unknown), } \\
\text { concanavalin A-like superfamily }\end{array}$ & 4135 & 1 & 1944 \\
\hline
\end{tabular}


Table A.8: continued (back to overview)

\begin{tabular}{|c|c|c|c|c|c|}
\hline \multirow{2}{*}{\multicolumn{2}{|c|}{$\frac{\text { COG ID }}{3802}$}} & \multirow{2}{*}{$\begin{array}{r}\text { DESCRIPTION } \\
\text { Uncharacterized protein }\end{array}$} & \multicolumn{3}{|c|}{$\mathrm{G}: \mathrm{E}: \mathrm{N} / \mathrm{L}: \mathrm{R}: \mathrm{N}$} \\
\hline & & & 3183 & 1 & 2140 \\
\hline $\mathrm{T}$ & 0467 & $\begin{array}{l}\text { RecA-superfamily ATPase, } \\
\text { KaiC/GvpD/RAD55 family }\end{array}$ & 2862 & 1 & 2540 \\
\hline $\mathrm{U}$ & 4618 & $\begin{array}{l}\text { ABC-type protease/lipase } \\
\text { transport system, ATPase and } \\
\text { permease components }\end{array}$ & 1 & 2016 & 1290 \\
\hline
\end{tabular}

Table A.9: Changes in COGs at the LCA of Loktanella vestfoldensis DSM 16212 and SKA 53 (a polar and temperate isolate, respectively). See description of Table A.6 for detailed information. (back to overview)

\begin{tabular}{|c|c|c|c|c|c|}
\hline & COG ID & DESCRIPTION & G:E & $\mathrm{N} / \mathrm{L}: \mathrm{H}$ & $\mathrm{R}: \mathrm{N}$ \\
\hline \multirow{7}{*}{ C } & O778 & Nitroreductase & 1 & 1844 & 150 \\
\hline & 1182 & $\begin{array}{l}\text { FMN-dependent } \\
\text { NADH-azoreductase }\end{array}$ & 3781 & 1 & 708 \\
\hline & 1251 & $\begin{array}{l}\text { NAD }(P) H \text {-nitrite reductase, } \\
\text { large subunit }\end{array}$ & 15 & 1 & $110^{3}$ \\
\hline & 1319 & $\begin{array}{l}\text { CO or xanthine dehydrogenase, } \\
\text { FAD-binding subunit }\end{array}$ & 1 & 135 & 107 \\
\hline & 2326 & $\begin{array}{l}\text { Polyphosphate kinase } 2, \mathrm{PPK}_{2} \\
\text { family }\end{array}$ & 1 & 3556 & 397 \\
\hline & 3658 & Cytochrome b & 72 & 1 & $1910^{3}$ \\
\hline & 4147 & $\begin{array}{l}\mathrm{Na}^{+}\left(\text {or } \mathrm{H}^{+}\right) / \text {acetate symporter } \\
\text { ActP }\end{array}$ & 16 & 1 & $9 \quad 10^{3}$ \\
\hline \multirow[t]{2}{*}{$\mathrm{D}$} & 1196 & $\begin{array}{l}\text { Chromosome segregation } \\
\text { ATPase }\end{array}$ & 1 & 8422 & 6645 \\
\hline & 4942 & $\begin{array}{l}\text { Septal ring factor EnvC, activator } \\
\text { of murein hydrolases AmiA and } \\
\text { AmiB }\end{array}$ & 1 & 4222 & 596 \\
\hline & 0069 & Glutamate synthase domain 2 & 1 & 1345 & 304 \\
\hline & 0308 & Aminopeptidase $\mathrm{N}$ & 455 & 1 & $6 \quad 10^{4}$ \\
\hline & 0509 & $\begin{array}{l}\text { Glycine cleavage system } \mathrm{H} \\
\text { protein (lipoate-binding) }\end{array}$ & 61 & 1 & $210^{3}$ \\
\hline E & 1003 & $\begin{array}{l}\text { Glycine cleavage system protein } \\
\text { P (pyridoxal-binding), } \\
\text { C-terminal domain }\end{array}$ & 45 & 1 & $5 \quad 10^{3}$ \\
\hline
\end{tabular}


Table A.9: continued (back to overview)

\begin{tabular}{|c|c|c|c|c|c|}
\hline & COG ID & DESCRIPTION & G:E & $\mathrm{N} / \mathrm{L}: 1$ & $\mathrm{R}: \mathrm{N}$ \\
\hline & 1063 & $\begin{array}{l}\text { Threonine dehydrogenase or } \\
\text { related } \mathrm{Zn} \text {-dependent } \\
\text { dehydrogenase }\end{array}$ & 1 & 848 & 810 \\
\hline & 1247 & $\begin{array}{l}\text { L-amino acid N-acyltransferase } \\
\text { YncA }\end{array}$ & 45 & 1 & $210^{3}$ \\
\hline & 1410 & $\begin{array}{l}\text { Methionine synthase I, } \\
\text { cobalamin-binding domain }\end{array}$ & 1 & 176 & $21 \quad 10^{4}$ \\
\hline & 1770 & Protease II & 14 & 1 & $1110^{3}$ \\
\hline & 1834 & $\begin{array}{l}\text { N-Dimethylarginine } \\
\text { dimethylaminohydrolase }\end{array}$ & 11 & 1 & $6 \quad 10^{3}$ \\
\hline & 2355 & $\begin{array}{l}\text { Zn-dependent dipeptidase, } \\
\text { microsomal dipeptidase } \\
\text { homolog }\end{array}$ & 367 & 1 & 20 \\
\hline & 2755 & $\begin{array}{l}\text { Lysophospholipase } \mathrm{L} 1 \text { or related } \\
\text { esterase }\end{array}$ & 1 & 793 & 102 \\
\hline & 4160 & $\begin{array}{l}\text { ABC-type arginine/histidine } \\
\text { transport system, permease } \\
\text { component }\end{array}$ & 1 & 268 & 102 \\
\hline & 4215 & $\begin{array}{l}\text { ABC-type arginine transport } \\
\text { system, permease component }\end{array}$ & 1 & 33 & $1310^{4}$ \\
\hline \multirow[t]{2}{*}{$\mathrm{F}$} & 0563 & $\begin{array}{l}\text { Adenylate kinase or related } \\
\text { kinase }\end{array}$ & 1 & 6621 & 4071 \\
\hline & o788 & $\begin{array}{l}\text { Formyltetrahydrofolate } \\
\text { hydrolase }\end{array}$ & 267 & 1 & 161 \\
\hline \multirow{7}{*}{ G } & 0235 & $\begin{array}{l}\text { Ribulose-5-phosphate } \\
\text { 4-epimerase/Fuculose-1- } \\
\text { phosphate } \\
\text { aldolase }\end{array}$ & 48 & 1 & 3 \\
\hline & 0246 & $\begin{array}{l}\text { Mannitol-1-phosphate/altronate } \\
\text { dehydrogenases }\end{array}$ & 1 & 5269 & 328 \\
\hline & 0297 & Glycogen synthase & 29 & 1 & 22 \\
\hline & 0362 & $\begin{array}{l}\text { 6-phosphogluconate } \\
\text { dehydrogenase }\end{array}$ & 188 & 1 & $310^{4}$ \\
\hline & 0366 & Glycosidase & 1 & 148 & 63 \\
\hline & 0448 & $\begin{array}{l}\text { ADP-glucose } \\
\text { pyrophosphorylase }\end{array}$ & 4533 & 1 & 3258 \\
\hline & 0580 & $\begin{array}{l}\text { Glycerol uptake facilitator and } \\
\text { related aquaporins (Major } \\
\text { Intrinsic Protein Family) }\end{array}$ & 1032 & 1 & 805 \\
\hline
\end{tabular}


Table A.9: continued (back to overview)

\begin{tabular}{|c|c|c|c|c|c|}
\hline \multirow{2}{*}{\multicolumn{2}{|c|}{$\begin{array}{c}\text { COG ID } \\
0637\end{array}$}} & \multirow{2}{*}{\begin{tabular}{l}
\multicolumn{1}{c}{ DESCRIPTION } \\
$\beta$-phosphoglucomutase or \\
related phosphatase, HAD \\
superfamily
\end{tabular}} & \multicolumn{3}{|c|}{$\mathrm{G}: \mathrm{E}: \mathrm{N} / \mathrm{L}: \mathrm{R}: \mathrm{N}$} \\
\hline & & & 221 & 1 & 15 \\
\hline & 0698 & $\begin{array}{l}\text { Ribose 5-phosphate isomerase } \\
\text { RpiB }\end{array}$ & 116 & 1 & $60 \quad 10^{3}$ \\
\hline & 2211 & $\begin{array}{l}\mathrm{Na}^{+} / \text {melibiose symporter or } \\
\text { related transporter }\end{array}$ & 1 & 1278 & 724 \\
\hline & 2220 & $\begin{array}{l}\text { L-ascorbate metabolism protein } \\
\text { UlaG, } \beta \text {-lactamase superfamily }\end{array}$ & & 1 & 3 \\
\hline & 2943 & Membrane glycosyltransferase & 80 & 1 & 53 \\
\hline & 3622 & Hydroxypyruvate isomerase & 145 & 1 & 6 \\
\hline & 4813 & Trehalose utilization protein & 55 & 1 & $4310^{3}$ \\
\hline \multirow{3}{*}{$\mathrm{H}$} & 0414 & Panthothenate synthetase & 72 & 1 & $210^{3}$ \\
\hline & 0432 & $\begin{array}{l}\text { Thiamin phosphate synthase } \\
\text { YjbQ, UPFoo47 family }\end{array}$ & 37 & 1 & $2910^{3}$ \\
\hline & 4032 & $\begin{array}{l}\text { Sulfopyruvate decarboxylase, } \\
\text { TPP-binding subunit (coenzyme } \\
\text { M biosynthesis) }\end{array}$ & 69 & 1 & $3710^{3}$ \\
\hline \multirow{3}{*}{ I } & 0584 & $\begin{array}{l}\text { Glycerophosphoryl diester } \\
\text { phosphodiesterase }\end{array}$ & 1 & 6720 & 6281 \\
\hline & 3000 & $\begin{array}{l}\text { Sterol desaturase/sphingolipid } \\
\text { hydroxylase, fatty acid } \\
\text { hydroxylase superfamily }\end{array}$ & 265 & 1 & 129 \\
\hline & 4850 & $\begin{array}{l}\text { Phosphatidate phosphatase } \\
\text { APP1 }\end{array}$ & & 1 & $8810^{3}$ \\
\hline $\mathrm{J}$ & 3719 & Ribonuclease I & 369 & 1 & $5 \quad 10^{4}$ \\
\hline \multirow{4}{*}{ K } & 0553 & $\begin{array}{l}\text { Superfamily II DNA or RNA } \\
\text { helicase, SNF2 family }\end{array}$ & 49 & 1 & $27 \quad 10^{3}$ \\
\hline & o864 & $\begin{array}{l}\text { Metal-responsive transcriptional } \\
\text { regulator, contains } \\
\text { CopG/Arc/MetJ DNA-binding } \\
\text { domain }\end{array}$ & 55 & 1 & $9 \quad 10^{4}$ \\
\hline & 2186 & $\begin{array}{l}\text { DNA-binding transcriptional } \\
\text { regulator, FadR family }\end{array}$ & 1 & \begin{tabular}{|l|}
8408 \\
\end{tabular} & 7368 \\
\hline & 3070 & $\begin{array}{l}\text { Transcriptional regulator of } \\
\text { competence genes, TfoX/Sxy } \\
\text { family }\end{array}$ & 96 & 1 & $50 \quad 10^{3}$ \\
\hline
\end{tabular}


Table A.9: continued (back to overview)

\begin{tabular}{|c|c|c|c|c|c|}
\hline & COG ID & DESCRIPTION & $\mathrm{G}: \mathrm{E}$ & N/L:I & $\mathrm{R}: \mathrm{N}$ \\
\hline \multirow{7}{*}{$\mathrm{L}$} & 0350 & $\begin{array}{l}\text { O6-methylguanine-DNA- } \\
\text { protein-cysteine } \\
\text { methyltransferase }\end{array}$ & 1 & 45 & $110^{3}$ \\
\hline & 0553 & $\begin{array}{l}\text { Superfamily II DNA or RNA } \\
\text { helicase, SNF2 family }\end{array}$ & 49 & 1 & $2710^{3}$ \\
\hline & 1484 & DNA replication protein DnaC & 637 & 1 & 118 \\
\hline & 2176 & $\begin{array}{l}\text { DNA polymerase III, alpha } \\
\text { subunit (gram-positive type) }\end{array}$ & 7499 & 1 & 4127 \\
\hline & 2827 & $\begin{array}{l}\text { Predicted endonuclease, } \\
\text { GIY-YIG superfamily }\end{array}$ & 103 & 1 & $3710^{3}$ \\
\hline & 3569 & DNA topoisomerase IB & 70 & 1 & $38 \quad 10^{3}$ \\
\hline & 4912 & $\begin{array}{l}\text { 3-methyladenine DNA } \\
\text { glycosylase AlkD }\end{array}$ & 538 & 1 & $1210^{3}$ \\
\hline \multirow{4}{*}{ M } & 0399 & $\begin{array}{l}\text { dTDP-4-amino-4,6- } \\
\text { dideoxygalactose } \\
\text { transaminase }\end{array}$ & 57 & 3 & 1 \\
\hline & 2943 & Membrane glycosyltransferase & 80 & 1 & 53 \\
\hline & 2989 & $\begin{array}{l}\text { Murein L,D-transpeptidase } \\
\text { YcbB/YkuD }\end{array}$ & 130 & 1 & $1 \quad 10^{5}$ \\
\hline & 5622 & $\begin{array}{l}\text { Protein required for attachment } \\
\text { to host cells }\end{array}$ & 105 & 1 & $58 \quad 10^{3}$ \\
\hline \multirow{9}{*}{$\mathrm{O}$} & 0225 & $\begin{array}{l}\text { Peptide methionine sulfoxide } \\
\text { reductase MsrA }\end{array}$ & 1 & 102 & $210^{3}$ \\
\hline & 0846 & $\begin{array}{l}\text { NAD-dependent protein } \\
\text { deacetylase, SIR } 2 \text { family }\end{array}$ & 100 & 1 & $51 \quad 10^{3}$ \\
\hline & 1858 & Cytochrome c peroxidase & 1 & 8159 & 4065 \\
\hline & 2020 & $\begin{array}{l}\text { Protein-S-isoprenylcysteine } \\
\text { O-methyltransferase Ste14 }\end{array}$ & 1 & 136 & 110 \\
\hline & 2135 & $\begin{array}{l}\text { Putative SOS } \\
\text { response-associated peptidase } \\
\text { YedK }\end{array}$ & 3258 & 1 & 97 \\
\hline & 2170 & $\begin{array}{l}\text { Gamma-glutamyl:cysteine ligase } \\
\text { YbdK, ATP-grasp superfamily }\end{array}$ & 14 & 1 & $1010^{3}$ \\
\hline & 2370 & $\begin{array}{l}\text { Hydrogenase/urease accessory } \\
\text { protein HupE }\end{array}$ & 14 & 1 & $910^{3}$ \\
\hline & 0310 & $\begin{array}{l}\text { ABC-type } \mathrm{Co}^{2+} \text { transport } \\
\text { system, permease component }\end{array}$ & 298 & 1 & $3410^{3}$ \\
\hline & 0475 & $\begin{array}{l}\text { Kef-type } \mathrm{K}^{+} \text {transport system, } \\
\text { membrane component KefB }\end{array}$ & 1 & 14 & 6 \\
\hline
\end{tabular}


Table A.9: continued (back to overview)

\begin{tabular}{|c|c|c|c|c|c|}
\hline & COG ID & DESCRIPTION & $\mathrm{G}: \mathrm{E}$ & N/L:I & $\mathrm{R}: \mathrm{N}$ \\
\hline & 0704 & Phosphate uptake regulator & 1 & 37 & $1310^{4}$ \\
\hline & 1814 & $\begin{array}{l}\text { Predicted } \mathrm{Fe}^{2+} / \mathrm{Mn}^{2+} \\
\text { transporter, VIT1 } / \mathrm{CCC}_{1} \text { family }\end{array}$ & 8121 & 1 & 7623 \\
\hline & 1840 & $\begin{array}{l}\text { ABC-type } \mathrm{Fe}^{3+} \text { transport } \\
\text { system, periplasmic component }\end{array}$ & 1 & 1569 & 108 \\
\hline & 2116 & $\begin{array}{l}\text { Formate/nitrite transporter } \\
\text { FocA, FNT family }\end{array}$ & 88 & 1 & $48 \quad 10^{3}$ \\
\hline & 2215 & $\begin{array}{l}\text { ABC-type nickel/cobalt efflux } \\
\text { system, permease component } \\
\text { RcnA }\end{array}$ & 33 & 1 & 31 \\
\hline & 2608 & Copper chaperone CopZ & 2393 & 1 & 1421 \\
\hline & 2847 & Copper(I)-binding protein & 1 & 12 & $910^{3}$ \\
\hline & 3197 & $\begin{array}{l}\text { Uncharacterized protein, } \\
\text { possibly involved in nitrogen } \\
\text { fixation }\end{array}$ & 7344 & 1 & 480 \\
\hline & 3221 & $\begin{array}{l}\text { ABC-type } \\
\text { phosphate/phosphonate } \\
\text { transport system, periplasmic } \\
\text { component }\end{array}$ & 1 & 8027 & 5756 \\
\hline & 3454 & $\begin{array}{l}\alpha \text {-D-ribose } \\
\text { 1-methylphosphonate } \\
\text { 5-triphosphate diphosphatase } \\
\text { PhnM }\end{array}$ & 4 & 1 & 1 \\
\hline & 3720 & $\begin{array}{l}\text { Putative heme degradation } \\
\text { protein }\end{array}$ & 6749 & 1 & 5850 \\
\hline & 4521 & $\begin{array}{l}\text { ABC-type taurine transport } \\
\text { system, periplasmic component }\end{array}$ & 69 & 1 & $5210^{3}$ \\
\hline & $455^{8}$ & $\begin{array}{l}\text { ABC-type hemin transport } \\
\text { system, periplasmic component }\end{array}$ & 8006 & 1 & 7784 \\
\hline & 4559 & $\begin{array}{l}\text { ABC-type hemin transport } \\
\text { system, ATPase component }\end{array}$ & 15 & 1 & $10 \quad 10^{3}$ \\
\hline & 4651 & $\begin{array}{l}\text { Predicted Kef-type } \mathrm{K}^{+} \text {transport } \\
\text { protein, } \mathrm{K}^{+} / \mathrm{H}^{+} \text {antiporter } \\
\text { domain }\end{array}$ & 199 & 1 & $3610^{3}$ \\
\hline \multirow{3}{*}{ Q } & 1647 & Esterase/lipase & 46 & 1 & $3510^{3}$ \\
\hline & 2931 & $\begin{array}{l}\mathrm{Ca}^{2+} \text {-binding protein, } \mathrm{RTX} \\
\text { toxin-related }\end{array}$ & 33 & 1 & 1 \\
\hline & 3509 & $\begin{array}{l}\text { Poly(3-hydroxybutyrate) } \\
\text { depolymerase }\end{array}$ & 40 & 1 & 40 \\
\hline
\end{tabular}


Table A.9: continued (back to overview)

\begin{tabular}{|c|c|c|c|c|c|}
\hline & COG ID & DESCRIPTION & G:E & $\mathrm{N} / \mathrm{L}: 1$ & $\mathrm{R}: \mathrm{N}$ \\
\hline \multirow{17}{*}{$\mathrm{R}$} & 0384 & $\begin{array}{l}\text { Predicted epimerase } \\
\text { YddE/YHI9, PhzF superfamily }\end{array}$ & 1528 & 1 & 76 \\
\hline & 0637 & $\begin{array}{l}\beta \text {-phosphoglucomutase or } \\
\text { related phosphatase, HAD } \\
\text { superfamily }\end{array}$ & 221 & 1 & 15 \\
\hline & 1063 & $\begin{array}{l}\text { Threonine dehydrogenase or } \\
\text { related Zn-dependent } \\
\text { dehydrogenase }\end{array}$ & 1 & 848 & 810 \\
\hline & 1739 & $\begin{array}{l}\text { Putative translation regulator, } \\
\text { IMPACT (imprinted ancient) } \\
\text { protein family }\end{array}$ & 31 & 1 & $310^{3}$ \\
\hline & 1741 & $\begin{array}{l}\text { Redox-sensitive bicupin YhaK, } \\
\text { pirin superfamily }\end{array}$ & 1 & 3 & 3 \\
\hline & 1765 & $\begin{array}{l}\text { Uncharacterized OsmC-related } \\
\text { protein }\end{array}$ & 6585 & 1 & 5860 \\
\hline & 1917 & $\begin{array}{l}\text { Cupin domain protein related to } \\
\text { quercetin dioxygenase }\end{array}$ & 490 & 1 & 26 \\
\hline & 2249 & $\begin{array}{l}\text { Putative NADPH-quinone } \\
\text { reductase (modulator of drug } \\
\text { activity B) }\end{array}$ & 26 & 1 & 18 \\
\hline & 2304 & $\begin{array}{l}\text { Secreted protein containing } \\
\text { bacterial Ig-like domain and } \\
\text { vWFA domain }\end{array}$ & 48 & 1 & $4 \quad 10^{3}$ \\
\hline & 2321 & Predicted metalloprotease & 12 & 1 & $9 \quad 10^{3}$ \\
\hline & 3021 & $\begin{array}{l}\text { Uncharacterized conserved } \\
\text { protein YafD, endonuclease/ex- } \\
\text { onuclease/phosphatase (EEP) } \\
\text { superfamily }\end{array}$ & 336 & 1 & 216 \\
\hline & 3153 & $\begin{array}{l}\text { Predicted N-acetyltransferase } \\
\text { YhbS }\end{array}$ & 1 & 3007 & 118 \\
\hline & 3214 & $\begin{array}{l}\text { Uncharacterized conserved } \\
\text { protein YcaQ, contains winged } \\
\text { helix DNA-binding domain }\end{array}$ & 7076 & 1 & 235 \\
\hline & 3217 & $\begin{array}{l}\text { Uncharacterized conserved } \\
\text { protein YcbX, contains MOSC } \\
\text { and Fe-S domains }\end{array}$ & 67 & 1 & $40 \quad 10^{3}$ \\
\hline & 3360 & Flavin-binding protein dodecin & 116 & 1 & $6610^{3}$ \\
\hline & 3380 & $\begin{array}{l}\text { Predicted NAD/FAD-dependent } \\
\text { oxidoreductase }\end{array}$ & 500 & 1 & 178 \\
\hline & 3393 & $\begin{array}{l}\text { Predicted acetyltransferase, } \\
\text { GNAT family }\end{array}$ & 47 & 1 & $5 \quad 10^{3}$ \\
\hline
\end{tabular}


Table A.9: continued (back to overview)

\begin{tabular}{|c|c|c|c|c|c|}
\hline COG ID & DESCRIPTION & G:E & $\mathrm{N} / \mathrm{L}:$ & $\mathrm{R}: \mathrm{N}$ & \\
\hline 3450 & $\begin{array}{l}\text { Predicted enzyme of the cupin } \\
\text { superfamily }\end{array}$ & 74 & 1 & 37 & $10^{3}$ \\
\hline 3576 & $\begin{array}{l}\text { Predicted } \\
\text { flavin-nucleotide-binding } \\
\text { protein, pyridoxine } 5^{\prime} \text {-phosphate } \\
\text { oxidase superfamily }\end{array}$ & 307 & 1 & 12 & \\
\hline 3828 & $\begin{array}{l}\text { Type } 1 \text { glutamine } \\
\text { amidotransferase (GATase1)-like } \\
\text { domain }\end{array}$ & 65 & 1 & 11 & $10^{3}$ \\
\hline 1295 & $\begin{array}{l}\text { Uncharacterized membrane } \\
\text { protein, BrkB/YihY/UPFo761 } \\
\text { family (not an RNase) }\end{array}$ & 1 & 895 & 480 & \\
\hline 2268 & $\begin{array}{l}\text { Uncharacterized membrane } \\
\text { protein YqiK, contains } \\
\text { Band7/PHB/SPFH domain }\end{array}$ & 79 & 1 & 29 & $10^{3}$ \\
\hline 2311 & $\begin{array}{l}\text { Uncharacterized membrane } \\
\text { protein YeiB }\end{array}$ & 62 & 1 & 17 & $10^{3}$ \\
\hline 2833 & $\begin{array}{l}\text { Uncharacterized conserved } \\
\text { protein, contains ferritin-like } \\
\text { DUF455 domain }\end{array}$ & 21 & 1 & 1 & $10^{3}$ \\
\hline 3108 & $\begin{array}{l}\text { Uncharacterized conserved } \\
\text { protein YcbK, DUF882 family }\end{array}$ & 1 & 33 & 13 & $10^{3}$ \\
\hline 3514 & $\begin{array}{l}\text { Uncharacterized conserved } \\
\text { protein, DUF } 4415 \text { family }\end{array}$ & 21 & 1 & 15 & $10^{3}$ \\
\hline 3544 & $\begin{array}{l}\text { Uncharacterized conserved } \\
\text { protein, } \mathrm{DUF}_{305} \text { family }\end{array}$ & 249 & 1 & 187 & \\
\hline 3673 & $\begin{array}{l}\text { Uncharacterized protein, } \\
\text { PA2063/DUF2235 family }\end{array}$ & 1 & 434 & 236 & \\
\hline 3739 & $\begin{array}{l}\text { Uncharacterized membrane } \\
\text { protein YoaT, DUF817 family }\end{array}$ & 90 & 1 & 9 & $10^{3}$ \\
\hline 3788 & $\begin{array}{l}\text { Uncharacterized membrane } \\
\text { protein } Y e c N, \text { MAPEG domain }\end{array}$ & 2783 & 1 & 1563 & \\
\hline 3832 & $\begin{array}{l}\text { Uncharacterized conserved } \\
\text { protein YndB, AHSA1/START } \\
\text { domain }\end{array}$ & 1 & 5 & 2 & \\
\hline 3921 & $\begin{array}{l}\text { Uncharacterized conserved } \\
\text { protein }\end{array}$ & 1 & 28 & 2 & \\
\hline 4093 & Uncharacterized protein & 29 & 1 & 2 & $10^{4}$ \\
\hline 4275 & Uncharacterized protein & 90 & 1 & 8 & $10^{3}$ \\
\hline
\end{tabular}


Table A.9: continued (back to overview)

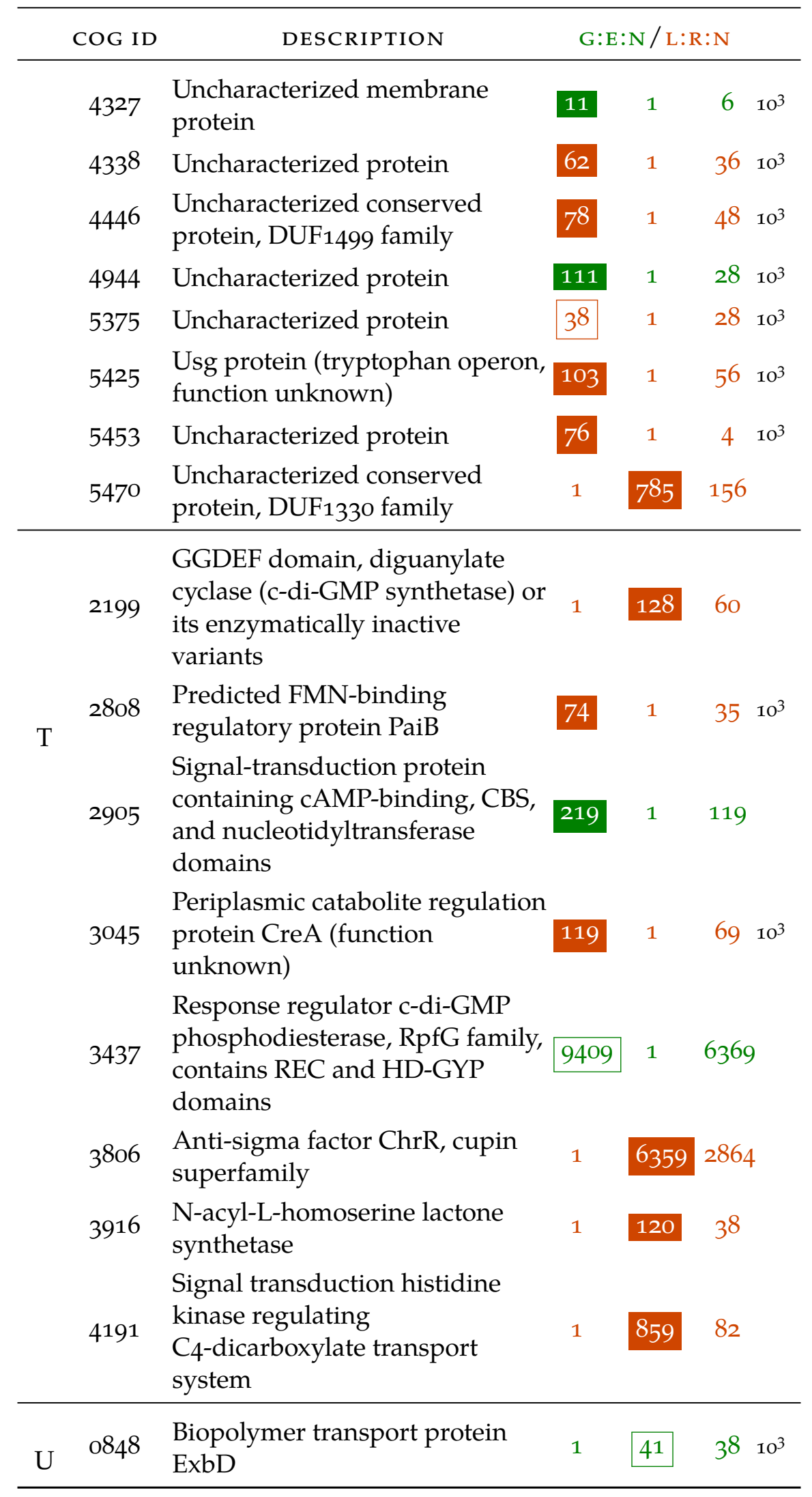


Table A.9: continued (back to overview)

\begin{tabular}{|c|c|c|c|c|c|}
\hline \multicolumn{2}{|r|}{ COG ID } & DESCRIPTION & \multicolumn{3}{|c|}{$\mathrm{G}: \mathrm{E}: \mathrm{N} / \mathrm{L}: \mathrm{R}: \mathrm{N}$} \\
\hline & 5010 & $\begin{array}{l}\text { Flp pilus assembly protein TadD, } \\
\text { contains TPR repeats }\end{array}$ & 21 & 1 & 1 \\
\hline \multirow[t]{2}{*}{$\mathrm{V}$} & 1566 & $\begin{array}{l}\text { Multidrug resistance efflux } \\
\text { pump }\end{array}$ & 1 & 44 & 43 \\
\hline & 1680 & $\begin{array}{l}\text { CubicO group peptidase, } \\
\beta \text {-lactamase class } C \text { family }\end{array}$ & 1 & 389 & 249 \\
\hline W & 5010 & $\begin{array}{l}\text { Flp pilus assembly protein TadD, } \\
\text { contains TPR repeats }\end{array}$ & 21 & 1 & 1 \\
\hline$X$ & 3436 & Transposase & 356 & 1 & 73 \\
\hline
\end{tabular}

Table A.10: Changes in COGs at the LCA of O. temperatus and O. ascidiaceicola. See description of Table A.6 for detailed information. (back to overview)

\begin{tabular}{|c|c|c|c|c|c|}
\hline & COG ID & DESCRIPTION & & $\mathrm{N} / \mathrm{L}: \mathrm{I}$ & $: \mathrm{N}$ \\
\hline \multirow{9}{*}{$\mathrm{C}$} & 0644 & Dehydrogenase (flavoprotein) & 1 & 5737 & 1964 \\
\hline & 0785 & $\begin{array}{l}\text { Cytochrome c biogenesis } \\
\text { protein CcdA }\end{array}$ & 1 & 60 & $10^{3}$ \\
\hline & 1049 & Aconitase B & 80 & 1 & $1110^{3}$ \\
\hline & 1053 & $\begin{array}{l}\text { Succinate dehydrogenase/ } \\
\text { fumarate reductase, flavoprotein } \\
\text { subunit }\end{array}$ & 1 & 71 & $3410^{3}$ \\
\hline & 2041 & $\begin{array}{l}\text { Periplasmic DMSO/TMAO } \\
\text { reductase YedYZ, } \\
\text { molybdopterin-dependent } \\
\text { catalytic subunit }\end{array}$ & 1 & 15 & $810^{3}$ \\
\hline & 2055 & $\begin{array}{l}\text { Malate/lactate/ureidoglycolate } \\
\text { dehydrogenase, } \mathrm{LDH}_{2} \text { family }\end{array}$ & 1 & 2559 & 1445 \\
\hline & 3258 & Cytochrome c & 31 & 1 & $10 \quad 10^{3}$ \\
\hline & 4106 & $\begin{array}{l}\text { Trans-aconitate } \\
\text { methyltransferase }\end{array}$ & 1 & 13 & 7 \\
\hline & 4736 & $\begin{array}{l}\text { Cbb3-type cytochrome oxidase, } \\
\text { subunit } 3\end{array}$ & 15 & 1 & $10^{4}$ \\
\hline \multirow[t]{2}{*}{$\mathrm{D}$} & 1475 & $\begin{array}{l}\text { Chromosome segregation } \\
\text { protein SpooJ, contains ParB-like } \\
\text { nuclease domain }\end{array}$ & 1 & 35 & $1210^{3}$ \\
\hline & 4942 & $\begin{array}{l}\text { Septal ring factor EnvC, activator } \\
\text { of murein hydrolases } \\
\text { AmiA and AmiB }\end{array}$ & 1 & 13 & $310^{3}$ \\
\hline
\end{tabular}


Table A.10: continued (back to overview)

\begin{tabular}{|c|c|c|c|c|c|c|}
\hline & COG ID & DESCRIPTION & G:E & $\mathrm{N} / \mathrm{L}$ & $\mathrm{R}: \mathrm{N}$ & \\
\hline \multirow{8}{*}{$\mathrm{E}$} & 0112 & $\begin{array}{l}\text { Glycine/serine } \\
\text { hydroxymethyltransferase }\end{array}$ & 1 & 12 & 3 & $10^{4}$ \\
\hline & 0531 & Amino acid transporter & 13 & 1 & 3 & $10^{4}$ \\
\hline & 1246 & $\begin{array}{l}\text { N-acetylglutamate synthase or } \\
\text { related acetyltransferase, } \\
\text { GNAT family }\end{array}$ & 1362 & 1 & 724 & \\
\hline & 1247 & $\begin{array}{l}\text { L-amino acid } \\
\text { N-acyltransferase YncA }\end{array}$ & 1 & 4 & 2 & $10^{4}$ \\
\hline & 1363 & Putative aminopeptidase FrvX & 43 & 1 & 1 & $10^{4}$ \\
\hline & 1748 & $\begin{array}{l}\text { Saccharopine dehydrogenase, } \\
\text { NADP-dependent }\end{array}$ & 2670 & 1 & 1505 & \\
\hline & 2049 & Allophanate hydrolase subunit 1 & 4280 & 1 & 2656 & \\
\hline & 3191 & L-aminopeptidase/D-esterase & 17 & 1 & 2 & $10^{3}$ \\
\hline \multirow{13}{*}{ G } & 0297 & Glycogen synthase & 11 & 1 & 2 & $10^{4}$ \\
\hline & 0448 & $\begin{array}{l}\text { ADP-glucose } \\
\text { pyrophosphorylase }\end{array}$ & 11 & 1 & 1 & $10^{4}$ \\
\hline & O574 & $\begin{array}{l}\text { Phosphoenolpyruvate synthase/ } \\
\text { pyruvate phosphate dikinase }\end{array}$ & 1 & 20 & 7 & $10^{3}$ \\
\hline & 0580 & $\begin{array}{l}\text { Glycerol uptake facilitator and } \\
\text { related aquaporins (Major } \\
\text { Intrinsic Protein Family) }\end{array}$ & 1 & 17 & 5 & \\
\hline & 0726 & $\begin{array}{l}\text { Peptidoglycan/xylan/chitin } \\
\text { deacetylase, PgdA/CDA1 family }\end{array}$ & 32 & 1 & 20 & \\
\hline & 0837 & Glucokinase & 1 & 55 & 7 & $10^{5}$ \\
\hline & 1363 & Putative aminopeptidase FrvX & 43 & 1 & 1 & $10^{4}$ \\
\hline & 1940 & $\begin{array}{l}\text { Sugar kinase of the NBD } / \mathrm{HSP}_{70} \\
\text { family, may contain an } \\
\text { N-terminal HTH domain }\end{array}$ & 1 & 8 & 4 & \\
\hline & 2115 & Xylose isomerase & 25 & 1 & 4 & $10^{5}$ \\
\hline & 2211 & $\begin{array}{l}\mathrm{Na}^{+} / \text {melibiose symporter or } \\
\text { related transporter }\end{array}$ & 98 & 10 & 1 & \\
\hline & 2942 & $\begin{array}{l}\text { Mannose or cellobiose } \\
\text { epimerase, } \\
\text { N-acyl-D-glucosamine } \\
\text { 2-epimerase family }\end{array}$ & 18 & 1 & 8 & $10^{3}$ \\
\hline & 4124 & $\beta$-mannanase & 37 & 1 & 5 & $10^{3}$ \\
\hline & 4130 & $\begin{array}{l}\text { Predicted sugar epimerase, } \\
\text { xylose isomerase-like family }\end{array}$ & 6635 & 1 & 2434 & \\
\hline
\end{tabular}


Table A.10: continued (back to overview)

\begin{tabular}{|c|c|c|c|c|c|}
\hline & COG ID & DESCRIPTION & & N/L:R & $\mathrm{R}: \mathrm{N}$ \\
\hline & 4213 & $\begin{array}{l}\text { ABC-type xylose transport } \\
\text { system, periplasmic component }\end{array}$ & 14 & 1 & $110^{3}$ \\
\hline & 4214 & $\begin{array}{l}\text { ABC-type xylose transport } \\
\text { system, permease component }\end{array}$ & 14 & 1 & $110^{3}$ \\
\hline & 4573 & $\begin{array}{l}\text { Tagatose-1,6-bisphosphate } \\
\text { aldolase non-catalytic subunit } \\
\text { AgaZ/GatZ }\end{array}$ & 81 & 1 & $1610^{3}$ \\
\hline \multirow{6}{*}{$\mathrm{H}$} & 0054 & $\begin{array}{l}6,7 \text {-dimethyl-8-ribityllumazine } \\
\text { synthase (Riboflavin synthase } \\
\beta \text { chain) }\end{array}$ & 1 & 68 & $2110^{4}$ \\
\hline & 0189 & $\begin{array}{l}\text { Glutathione synthase/ } \\
\text { RimK-type ligase, ATP-grasp } \\
\text { superfamily }\end{array}$ & 1 & 2907 & 1358 \\
\hline & 0661 & $\begin{array}{l}\text { Predicted unusual protein } \\
\text { kinase regulating ubiquinone } \\
\text { biosynthesis, AarF/ABC } 1 / \mathrm{UbiB} \\
\text { family }\end{array}$ & 1 & 80 & $5 \quad 10^{3}$ \\
\hline & 1120 & $\begin{array}{l}\text { ABC-type } \\
\text { cobalamin } / \mathrm{Fe}^{3+} \text {-siderophores } \\
\text { transport system, ATPase } \\
\text { component }\end{array}$ & 31 & 1 & $8 \quad 10^{3}$ \\
\hline & 1335 & Nicotinamidase-related amidase & 1 & 14 & $5 \quad 10^{4}$ \\
\hline & 2141 & $\begin{array}{l}\text { Flavin-dependent } \\
\text { oxidoreductase, luciferase family } \\
\text { (includes alkanesulfonate } \\
\text { monooxygenase SsuD and } \\
\text { methylene } \\
\text { tetrahydromethanopterin } \\
\text { reductase) }\end{array}$ & 1 & 13 & $1010^{4}$ \\
\hline I & 1562 & Phytoene/squalene synthetase & 66 & 2 & 1 \\
\hline \multirow[t]{4}{*}{$\mathrm{J}$} & 0189 & $\begin{array}{l}\text { Glutathione } \\
\text { synthase/RimK-type ligase, } \\
\text { ATP-grasp superfamily }\end{array}$ & 1 & 2907 & 1358 \\
\hline & 1236 & $\begin{array}{l}\text { RNA processing exonuclease, } \\
\text { beta-lactamase fold, } \mathrm{Cft} 2 \text { family }\end{array}$ & 177 & 1 & $7 \quad 10^{4}$ \\
\hline & 1600 & $\begin{array}{l}\text { Epoxyqueuosine } \\
\text { reductase QueG (queuosine } \\
\text { biosynthesis) }\end{array}$ & 1 & 50 & $8 \quad 10^{5}$ \\
\hline & 1733 & $\begin{array}{l}\text { DNA-binding transcriptional } \\
\text { regulator, HxlR family }\end{array}$ & & 1 & 2950 \\
\hline
\end{tabular}

K 
Table A.10: continued (back to overview)

\begin{tabular}{|c|c|c|c|c|c|}
\hline \multirow{2}{*}{\multicolumn{2}{|c|}{$\begin{array}{l}\text { COG ID } \\
1940\end{array}$}} & \multirow{2}{*}{$\begin{array}{l}\text { DESCRIPTION } \\
\text { Sugar kinase of the NBD/HSP7O } \\
\text { family, may contain an } \\
\text { N-terminal HTH domain }\end{array}$} & \multicolumn{3}{|c|}{$\mathrm{G}: \mathrm{E}: \mathrm{N} / \mathrm{L}: \mathrm{R}: \mathrm{N}$} \\
\hline & & & 1 & 8 & 4 \\
\hline & 2315 & $\begin{array}{l}\text { Predicted DNA-binding protein } \\
\text { with 'double-wing' structural } \\
\text { motif, MmcQ/YjbR family }\end{array}$ & 55 & 1 & 22 \\
\hline & 3284 & $\begin{array}{l}\text { Transcriptional regulator of } \\
\text { acetoin/glycerol metabolism }\end{array}$ & 13 & 1 & $4 \quad 10^{3}$ \\
\hline & 3609 & $\begin{array}{l}\text { Transcriptional regulator, } \\
\text { contains Arc/MetJ-type RHH } \\
\text { (ribbon-helix-helix) } \\
\text { DNA-binding domain }\end{array}$ & 59 & 1 & $1210^{3}$ \\
\hline & 3829 & $\begin{array}{l}\text { Transcriptional regulator } \\
\text { containing PAS, AAA-type } \\
\text { ATPase, and DNA-binding } \\
\text { Fis domains }\end{array}$ & 9477 & 1 & 3704 \\
\hline & 4977 & $\begin{array}{l}\text { Transcriptional regulator GlxA } \\
\text { family, contains an amidase } \\
\text { domain and an AraC-type } \\
\text { DNA-binding HTH domain }\end{array}$ & 1 & 38 & $2810^{3}$ \\
\hline \multirow{7}{*}{$\mathrm{L}$} & 0415 & $\begin{array}{l}\text { Deoxyribodipyrimidine } \\
\text { photolyase }\end{array}$ & 45 & 1 & 1 \\
\hline & 0582 & Integrase & 528 & 1 & 55 \\
\hline & 0847 & $\begin{array}{l}\text { DNA polymerase III, } \epsilon \text { subunit } \\
\text { or related } 3^{\prime}-5 \text { ' exonuclease }\end{array}$ & 1 & 48 & $3210^{3}$ \\
\hline & 1201 & Lhr-like helicase & 60 & 1 & $210^{5}$ \\
\hline & 1793 & ATP-dependent DNA ligase & 96 & 1 & $1610^{3}$ \\
\hline & 2818 & $\begin{array}{l}\text { 3-methyladenine DNA } \\
\text { glycosylase Tag }\end{array}$ & 1 & 50 & $1310^{4}$ \\
\hline & 4973 & Site-specific recombinase XerC & 11 & 1 & $4 \quad 10^{3}$ \\
\hline \multirow{4}{*}{ M } & 0726 & $\begin{array}{l}\text { Peptidoglycan/xylan/chitin } \\
\text { deacetylase, PgdA/CDA1 family }\end{array}$ & 32 & 1 & 20 \\
\hline & 0767 & $\begin{array}{l}\text { ABC-type transporter Mla } \\
\text { maintaining outer membrane } \\
\text { lipid asymmetry, permease } \\
\text { component MlaE }\end{array}$ & 1 & 1760 & 377 \\
\hline & 1087 & UDP-glucose 4-epimerase & 1 & 9003 & 4981 \\
\hline & 1088 & $\begin{array}{l}\text { dTDP-D-glucose } \\
\text { 4,6-dehydratase }\end{array}$ & 1 & 17 & 3 \\
\hline
\end{tabular}


Table A.10: continued (back to overview)

\begin{tabular}{|c|c|c|c|c|c|}
\hline & COG ID & DESCRIPTION & G:E & $\mathrm{N} / \mathrm{L}: \mathrm{H}$ & $R: N$ \\
\hline & 1091 & $\begin{array}{l}\text { dTDP-4-dehydrorhamnose } \\
\text { reductase }\end{array}$ & 1 & 14 & 3 \\
\hline & 1127 & $\begin{array}{l}\text { ABC-type transporter Mla } \\
\text { maintaining outer membrane } \\
\text { lipid asymmetry, ATPase } \\
\text { component MlaF }\end{array}$ & 1 & 1760 & 377 \\
\hline & 1209 & $\begin{array}{l}\text { dTDP-glucose } \\
\text { pyrophosphorylase }\end{array}$ & 1 & 10 & 2 \\
\hline & 1463 & $\begin{array}{l}\text { ABC-type transporter Mla } \\
\text { maintaining outer membrane } \\
\text { lipid asymmetry, periplasmic } \\
\text { component MlaD }\end{array}$ & 1 & 1760 & 377 \\
\hline & 1538 & Outer membrane protein TolC & 1 & 44 & $5 \quad 10^{4}$ \\
\hline & 2222 & $\begin{array}{l}\text { Fructoselysine-6-P-deglycase } \\
\text { FrlB and related proteins with } \\
\text { duplicated sugar isomerase (SIS) } \\
\text { domain }\end{array}$ & 55 & 1 & 27 \\
\hline & 3524 & $\begin{array}{l}\text { Capsule polysaccharide export } \\
\text { protein KpsE/RkpR }\end{array}$ & 1 & 31 & $2210^{3}$ \\
\hline \multirow{10}{*}{$\mathrm{O}$} & 0443 & $\begin{array}{l}\text { Molecular chaperone DnaK } \\
\text { (HSP7o) }\end{array}$ & 1 & 68 & $2410^{3}$ \\
\hline & 0785 & $\begin{array}{l}\text { Cytochrome c biogenesis } \\
\text { protein CcdA }\end{array}$ & 1 & 60 & $5 \quad 10^{3}$ \\
\hline & 1305 & $\begin{array}{l}\text { Transglutaminase-like enzyme, } \\
\text { putative cysteine protease }\end{array}$ & 41 & 1 & 18 \\
\hline & 1858 & Cytochrome c peroxidase & 1 & 50 & $5 \quad 10^{3}$ \\
\hline & 2143 & Thioredoxin-related protein & 6995 & 1 & 5018 \\
\hline & 2170 & $\begin{array}{l}\text { Gamma-glutamyl:cysteine ligase } \\
\text { YbdK, ATP-grasp superfamily }\end{array}$ & 28 & 1 & $5 \quad 10^{3}$ \\
\hline & 3484 & $\begin{array}{l}\text { Predicted proteasome-type } \\
\text { protease }\end{array}$ & 98 & 1 & $310^{4}$ \\
\hline & 0025 & $\begin{array}{l}\text { NhaP-type } \mathrm{Na}^{+} / \mathrm{H}^{+} \text {or } \mathrm{K}^{+} / \mathrm{H}^{+} \\
\text {antiporter }\end{array}$ & 39 & 1 & 2 \\
\hline & 0288 & Carbonic anhydrase & 115 & 1 & $110^{6}$ \\
\hline & 0614 & $\begin{array}{l}\mathrm{ABC} \text {-type } \mathrm{Fe}^{3+} \text {-hydroxamate } \\
\text { transport system, periplasmic } \\
\text { component }\end{array}$ & 8970 & 1 & 2220 \\
\hline
\end{tabular}


Table A.10: continued (back to overview)

\begin{tabular}{|c|c|c|c|c|c|c|}
\hline & COG ID & DESCRIPTION & G:E & N/L & $\mathrm{R}: \mathrm{N}$ & \\
\hline & 1120 & $\begin{array}{l}\text { ABC-type } \\
\text { cobalamin } / \mathrm{Fe}^{3+} \text {-siderophores } \\
\text { transport system, ATPase } \\
\text { component }\end{array}$ & 31 & 1 & 8 & $10^{3}$ \\
\hline & 1629 & $\begin{array}{l}\text { Outer membrane receptor } \\
\text { proteins, mostly Fe transport }\end{array}$ & 362 & 1 & 45 & \\
\hline & 2346 & Truncated hemoglobin YjbI & 1961 & 1 & 1119 & \\
\hline & 2375 & $\begin{array}{l}\text { NADPH-dependent ferric } \\
\text { siderophore reductase, contains } \\
\text { FAD-binding and SIP domains }\end{array}$ & 54 & 1 & 42 & $10^{3}$ \\
\hline & 3454 & $\begin{array}{l}\alpha \text {-D-ribose } \\
\text { 1-methylphosphonate } \\
\text { 5-triphosphate } \\
\text { diphosphatase PhnM }\end{array}$ & 674 & 1 & 529 & \\
\hline & 3720 & $\begin{array}{l}\text { Putative heme degradation } \\
\text { protein }\end{array}$ & 2658 & 1 & 1570 & \\
\hline & $455^{8}$ & $\begin{array}{l}\text { ABC-type hemin transport } \\
\text { system, periplasmic component }\end{array}$ & 2156 & 1 & 1078 & \\
\hline & 4559 & $\begin{array}{l}\text { ABC-type hemin transport } \\
\text { system, ATPase component }\end{array}$ & 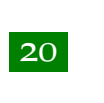 & 1 & 6 & $10^{3}$ \\
\hline & 4604 & $\begin{array}{l}\text { ABC-type enterochelin transport } \\
\text { system, ATPase component }\end{array}$ & & 1 & 10 & $10^{3}$ \\
\hline & 4605 & $\begin{array}{l}\text { ABC-type enterochelin transport } \\
\text { system, permease component }\end{array}$ & 9317 & 1 & 3566 & \\
\hline & 4606 & $\begin{array}{l}\text { ABC-type enterochelin transport } \\
\text { system, permease component }\end{array}$ & 1735 & 1 & 990 & \\
\hline & 4607 & $\begin{array}{l}\text { ABC-type enterochelin transport } \\
\text { system, periplasmic component }\end{array}$ & 4818 & 1 & 2015 & \\
\hline & 4638 & $\begin{array}{l}\text { Phenylpropionate dioxygenase } \\
\text { or related ring-hydroxylating } \\
\text { dioxygenase, large terminal } \\
\text { subunit }\end{array}$ & 1 & 13 & 12 & $10^{3}$ \\
\hline \multirow{5}{*}{ Q } & 1233 & $\begin{array}{l}\text { Phytoene dehydrogenase-related } \\
\text { protein }\end{array}$ & 276 & 1 & 63 & \\
\hline & 2130 & $\begin{array}{l}\text { NADPH-dependent curcumin } \\
\text { reductase CurA }\end{array}$ & 27 & 1 & 23 & $10^{4}$ \\
\hline & 3191 & L-aminopeptidase/D-esterase & 17 & 1 & 2 & $10^{3}$ \\
\hline & 1335 & Nicotinamidase-related amidase & 1 & 14 & 5 & $10^{4}$ \\
\hline & 1407 & $\begin{array}{l}\text { Metallophosphoesterase } \\
\text { superfamily enzyme }\end{array}$ & 60 & 1 & 2 & $10^{5}$ \\
\hline
\end{tabular}


Table A.10: continued (back to overview)

\begin{tabular}{|c|c|c|c|c|c|}
\hline COG ID & DESCRIPTION & $\mathrm{G}: \mathrm{E}$ & $: \mathrm{N} / \mathrm{L}:$ & $\mathrm{R}: \mathrm{N}$ & \\
\hline 1741 & $\begin{array}{l}\text { Redox-sensitive bicupin YhaK, } \\
\text { pirin superfamily }\end{array}$ & 1 & 277 & 144 & \\
\hline 2130 & $\begin{array}{l}\text { NADPH-dependent curcumin } \\
\text { reductase CurA }\end{array}$ & 27 & 1 & 23 & $10^{4}$ \\
\hline 2141 & $\begin{array}{l}\text { Flavin-dependent } \\
\text { oxidoreductase, luciferase family } \\
\text { (includes alkanesulfonate } \\
\text { monooxygenase SsuD and } \\
\text { methylene } \\
\text { tetrahydromethanopterin } \\
\text { reductase) }\end{array}$ & 1 & 13 & 10 & $10^{4}$ \\
\hline 2350 & $\begin{array}{l}\text { Uncharacterized conserved } \\
\text { protein YciI, contains a putative } \\
\text { active-site phosphohistidine }\end{array}$ & 1 & 15 & 2 & $10^{3}$ \\
\hline 3046 & $\begin{array}{l}\text { Uncharacterized protein related } \\
\text { to deoxyribodipyrimidine } \\
\text { photolyase }\end{array}$ & 55 & 1 & 2 & $10^{4}$ \\
\hline 3218 & $\begin{array}{l}\text { ABC-type uncharacterized } \\
\text { transport system, auxiliary } \\
\text { component }\end{array}$ & 20 & 1 & 3 & $10^{4}$ \\
\hline 3393 & $\begin{array}{l}\text { Predicted acetyltransferase, } \\
\text { GNAT family }\end{array}$ & 19 & 1 & 2 & $10^{4}$ \\
\hline 3453 & $\begin{array}{l}\text { Predicted phosphohydrolase, } \\
\text { protein tyrosine } \\
\text { phosphatase (PTP) superfamily, } \\
\text { DUF442 family }\end{array}$ & 1 & 18 & 8 & $10^{3}$ \\
\hline 3608 & Predicted deacylase & 4248 & 1 & 3419 & \\
\hline 3828 & $\begin{array}{l}\text { Type } 1 \text { glutamine } \\
\text { amidotransferase (GATase1)-like } \\
\text { domain }\end{array}$ & 20 & 1 & 3 & $10^{4}$ \\
\hline 4341 & Predicted HD phosphohydrolase & 2016 & 1 & 266 & \\
\hline 4638 & $\begin{array}{l}\text { Phenylpropionate dioxygenase } \\
\text { or related ring-hydroxylating } \\
\text { dioxygenase, large terminal } \\
\text { subunit }\end{array}$ & 1 & 13 & 12 & $10^{3}$ \\
\hline 4782 & $\begin{array}{l}\text { Esterase/lipase superfamily } \\
\text { enzyme }\end{array}$ & 42 & 1 & 11 & $10^{3}$ \\
\hline 4922 & $\begin{array}{l}\text { Predicted SnoaL-like aldol } \\
\text { condensation-catalyzing enzyme }\end{array}$ & 58 & 1 & 18 & $10^{3}$ \\
\hline
\end{tabular}


Table A.10: continued (back to overview)

\begin{tabular}{|c|c|c|c|c|c|}
\hline & COG ID & DESCRIPTION & G:E & $\mathrm{N} / \mathrm{L}: \mathrm{F}$ & $\mathrm{R}: \mathrm{N}$ \\
\hline \multirow{17}{*}{ S } & 1238 & $\begin{array}{l}\text { Uncharacterized membrane } \\
\text { protein YqaA, } \\
\text { SNARE-associated domain }\end{array}$ & 120 & 1 & $1 \quad 10^{4}$ \\
\hline & 1432 & $\begin{array}{l}\text { Uncharacterized conserved } \\
\text { protein, LabA/DUF88 family }\end{array}$ & 1 & 1048 & 725 \\
\hline & 2307 & $\begin{array}{l}\text { Uncharacterized conserved } \\
\text { protein, Alpha-E superfamily }\end{array}$ & 21 & 1 & $1 \quad 10^{4}$ \\
\hline & 2308 & $\begin{array}{l}\text { Uncharacterized conserved } \\
\text { protein, circularly permuted } \\
\text { ATPgrasp superfamily }\end{array}$ & 6328 & 1 & 2810 \\
\hline & 2862 & $\begin{array}{l}\text { Uncharacterized membrane } \\
\text { protein YqhA }\end{array}$ & 20 & 1 & $210^{5}$ \\
\hline & 3329 & $\begin{array}{l}\text { Uncharacterized conserved } \\
\text { protein }\end{array}$ & 17 & 1 & 3 \\
\hline & 3650 & $\begin{array}{l}\text { Uncharacterized membrane } \\
\text { protein }\end{array}$ & 2286 & 1 & 2050 \\
\hline & 3779 & $\begin{array}{l}\text { Uncharacterized conserved } \\
\text { protein YegJ, DUF2314 family }\end{array}$ & & 1 & $7 \quad 10^{4}$ \\
\hline & 3813 & Uncharacterized protein & 30 & 1 & $5 \quad 10^{4}$ \\
\hline & 3932 & $\begin{array}{l}\text { Uncharacterized conserved } \\
\text { protein }\end{array}$ & 6297 & 1 & 3021 \\
\hline & 4283 & Uncharacterized protein & 63 & 1 & $4 \quad 10^{4}$ \\
\hline & 4337 & Uncharacterized protein & 72 & 1 & $5 \quad 10^{4}$ \\
\hline & 4427 & Uncharacterized protein & 15 & 1 & $1 \quad 10^{4}$ \\
\hline & 4852 & $\begin{array}{l}\text { Uncharacterized membrane } \\
\text { protein }\end{array}$ & 7114 & 1 & 3467 \\
\hline & 5375 & Uncharacterized protein & 9 & 1 & $6 \quad 10^{4}$ \\
\hline & 5477 & $\begin{array}{l}\text { Predicted small integral } \\
\text { membrane protein }\end{array}$ & 29 & 1 & $1410^{4}$ \\
\hline & 5501 & Predicted secreted protein & 21 & 1 & $1010^{3}$ \\
\hline \multirow{3}{*}{$\mathrm{T}$} & 0661 & $\begin{array}{l}\text { Predicted unusual protein } \\
\text { kinase regulating ubiquinone } \\
\text { biosynthesis, AarF/ABC } 1 / \mathrm{UbiB} \\
\text { family }\end{array}$ & 1 & 80 & $5 \quad 10^{3}$ \\
\hline & 0784 & $\begin{array}{l}\text { CheY chemotaxis protein or a } \\
\text { CheY-like REC (receiver) domain }\end{array}$ & 2153 & 1 & 194 \\
\hline & 3228 & $\begin{array}{l}\text { Mlc titration factor MtfA, } \\
\text { regulates } p t s G \text { expression }\end{array}$ & 72 & 1 & $5 \quad 10^{4}$ \\
\hline
\end{tabular}


Table A.10: continued (back to overview)

\begin{tabular}{|c|c|c|c|c|c|}
\hline \multirow{2}{*}{\multicolumn{2}{|c|}{$\frac{\text { COG ID }}{3806}$}} & \multirow{2}{*}{$\begin{array}{l}\text { DESCRIPTION } \\
\text { Anti-sigma factor ChrR, cupin } \\
\text { superfamily }\end{array}$} & \multicolumn{3}{|c|}{$\mathrm{G}: \mathrm{E}: \mathrm{N} / \mathrm{L}: \mathrm{R}: \mathrm{N}$} \\
\hline & & & 1 & 42 & $310^{3}$ \\
\hline & 3829 & $\begin{array}{l}\text { Transcriptional regulator } \\
\text { containing PAS, AAA-type } \\
\text { ATPase, and DNA-binding } \\
\text { Fis domains }\end{array}$ & 9477 & 1 & 3704 \\
\hline & 3916 & $\begin{array}{l}\text { N-acyl-L-homoserine lactone } \\
\text { synthetase }\end{array}$ & 1 & 576 & 157 \\
\hline \multirow{5}{*}{$\mathrm{U}$} & 0342 & $\begin{array}{l}\text { Preprotein translocase } \\
\text { subunit SecD }\end{array}$ & 1 & 34 & $4 \quad 10^{5}$ \\
\hline & 0811 & $\begin{array}{l}\text { Biopolymer transport protein } \\
\text { ExbB/TolQ }\end{array}$ & 1 & 16 & $7 \quad 10^{3}$ \\
\hline & o848 & $\begin{array}{l}\text { Biopolymer transport protein } \\
\text { ExbD }\end{array}$ & 1 & 17 & $4 \quad 10^{4}$ \\
\hline & 1826 & $\begin{array}{l}\text { Sec-independent protein } \\
\text { translocase protein TatA }\end{array}$ & 2994 & 1 & 523 \\
\hline & 4618 & $\begin{array}{l}\text { ABC-type protease/lipase } \\
\text { transport system, ATPase and } \\
\text { permease components }\end{array}$ & 1 & 11 & $5 \quad 10^{3}$ \\
\hline \multirow{2}{*}{$x$} & 0582 & Integrase & 528 & 1 & 55 \\
\hline & 3668 & $\begin{array}{l}\text { Plasmid stabilization system } \\
\text { protein ParE }\end{array}$ & 812 & 1 & 760 \\
\hline
\end{tabular}

Table A.11: Changes in COGs at the LCA of the O. antarcticus type strain and the two PAMC isolates. See description of Table A.6 for detailed information. (back to overview)

\begin{tabular}{|c|c|c|c|c|c|}
\hline \multirow{3}{*}{ C } & \multirow{2}{*}{$\begin{array}{l}\text { COG ID } \\
0651\end{array}$} & \multirow{2}{*}{$\begin{array}{l}\text { DESCRIPTION } \\
\text { Formate hydrogenlyase subunit } \\
\text { 3/Multisubunit } \mathrm{Na}^{+} / \mathrm{H}^{+} \\
\text {antiporter, MnhD subunit }\end{array}$} & \multicolumn{3}{|c|}{$\mathrm{G}: \mathrm{E}: \mathrm{N} / \mathrm{L}: \mathrm{R}: \mathrm{N}$} \\
\hline & & & 1 & 35 & $1410^{3}$ \\
\hline & 1979 & $\begin{array}{l}\text { Alcohol dehydrogenase YqhD, } \\
\text { Fe-dependent ADH family }\end{array}$ & 5238 & 1 & 1850 \\
\hline $\mathrm{E}$ & 0165 & Argininosuccinate lyase & 1 & 54 & $3910^{3}$ \\
\hline $\mathrm{F}$ & 0647 & $\begin{array}{l}\text { Ribonucleotide } \\
\text { monophosphatase NagD, HAD } \\
\text { superfamily }\end{array}$ & 1 & 20 & $1110^{4}$ \\
\hline
\end{tabular}


Table A.11: continued (back to overview)

\begin{tabular}{|c|c|c|c|c|c|}
\hline & COG ID & DESCRIPTION & G:E & $\mathrm{N} / \mathrm{L}$ & $\mathrm{R}: \mathrm{N}$ \\
\hline \multirow{4}{*}{ G } & 1850 & $\begin{array}{l}\text { Ribulose } 1,5 \text {-bisphosphate } \\
\text { carboxylase, large subunit, or a } \\
\text { RuBisCO-like protein }\end{array}$ & 1166 & 1 & 1009 \\
\hline & 3734 & 2-keto-3-deoxy-galactonokinase & 1 & 194 & $46 \quad 10^{4}$ \\
\hline & 3958 & $\begin{array}{l}\text { Transketolase, C-terminal } \\
\text { subunit }\end{array}$ & 1168 & 1 & 1019 \\
\hline & 3959 & $\begin{array}{l}\text { Transketolase, N-terminal } \\
\text { subunit }\end{array}$ & 1168 & 1 & 1019 \\
\hline $\mathrm{H}$ & 2896 & $\begin{array}{l}\text { Molybdenum cofactor } \\
\text { biosynthesis enzyme MoaA }\end{array}$ & 1 & 67 & $3210^{4}$ \\
\hline I & 2230 & $\begin{array}{l}\text { Cyclopropane } \\
\text { fatty-acyl-phospholipid synthase } \\
\text { and related methyltransferases }\end{array}$ & 1 & 15 & $1210^{3}$ \\
\hline \multirow{3}{*}{ K } & 1321 & $\begin{array}{l}\text { Mn-dependent transcriptional } \\
\text { regulator, DtxR family }\end{array}$ & 5128 & 1 & 2232 \\
\hline & 3279 & $\begin{array}{l}\text { DNA-binding response } \\
\text { regulator, LytR/AlgR family }\end{array}$ & 1 & 5 & 5 \\
\hline & 5662 & $\begin{array}{l}\text { Transmembrane transcriptional } \\
\text { regulator (anti-sigma factor } \\
\text { RsiW) }\end{array}$ & 5442 & 1 & 2334 \\
\hline \multirow[t]{2}{*}{ M } & 1346 & $\begin{array}{l}\text { Putative effector of murein } \\
\text { hydrolase }\end{array}$ & 10 & 1 & 2 \\
\hline & 3713 & $\begin{array}{l}\text { Outer membrane scaffolding } \\
\text { protein for murein synthesis, } \\
\text { MipA/OmpV family }\end{array}$ & 2 & 1 & 1 \\
\hline \multirow[t]{5}{*}{$\mathrm{P}$} & 0651 & $\begin{array}{l}\text { Formate hydrogenlyase subunit } \\
3 / \text { Multisubunit } \mathrm{Na}^{+} / \mathrm{H}^{+} \\
\text {antiporter, MnhD subunit }\end{array}$ & 1 & 35 & $1410^{3}$ \\
\hline & 1320 & $\begin{array}{l}\text { Multisubunit } \mathrm{Na}^{+} / \mathrm{H}^{+} \\
\text {antiporter, MnhG subunit }\end{array}$ & 1 & 15 & $1310^{3}$ \\
\hline & 1863 & $\begin{array}{l}\text { Multisubunit } \mathrm{Na}^{+} / \mathrm{H}^{+} \\
\text {antiporter, MnhE subunit }\end{array}$ & 1 & 15 & $1310^{3}$ \\
\hline & 3002 & $\begin{array}{l}\text { Uncharacterized conserved } \\
\text { protein YbcC, } \\
\text { UPFo753/DUF2309 family }\end{array}$ & 9 & 1 & 2 \\
\hline & 3152 & $\begin{array}{l}\text { Uncharacterized membrane } \\
\text { protein YhaH, DUF805 family }\end{array}$ & 6 & 1 & 4 \\
\hline
\end{tabular}


Table A.11: continued (back to overview)

\begin{tabular}{|c|c|c|c|c|c|}
\hline \multirow{2}{*}{\multicolumn{2}{|c|}{$\begin{array}{l}\text { COG ID } \\
3205\end{array}$}} & \multirow{2}{*}{$\begin{array}{l}\text { DESCRIPTION } \\
\begin{array}{l}\text { Uncharacterized membrane } \\
\text { protein }\end{array}\end{array}$} & \multicolumn{3}{|c|}{$\mathrm{G}: \mathrm{E}: \mathrm{N} / \mathrm{L}: \mathrm{R}: \mathrm{N}$} \\
\hline & & & 6915 & 1 & 1791 \\
\hline & 3535 & $\begin{array}{l}\text { Uncharacterized conserved } \\
\text { protein, DUF917 family }\end{array}$ & 24 & 1 & 10 \\
\hline & 4728 & Uncharacterized protein & 3891 & 1 & 1533 \\
\hline & 5467 & Uncharacterized protein & 1 & 51 & $3210^{3}$ \\
\hline & 5587 & $\begin{array}{l}\text { Uncharacterized conserved } \\
\text { protein, DUF } 2461 \text { family }\end{array}$ & 2 & 1 & 2 \\
\hline $\mathrm{T}$ & 3279 & $\begin{array}{l}\text { DNA-binding response } \\
\text { regulator, LytR/AlgR family }\end{array}$ & 1 & 5 & 5 \\
\hline \multirow[t]{2}{*}{$\mathrm{U}$} & 0811 & $\begin{array}{l}\text { Biopolymer transport protein } \\
\text { ExbB/TolQ }\end{array}$ & 1 & 11 & $5 \quad 10^{3}$ \\
\hline & 0848 & $\begin{array}{l}\text { Biopolymer transport protein } \\
\text { ExbD }\end{array}$ & 1 & 70 & $3210^{3}$ \\
\hline V & 0610 & $\begin{array}{l}\text { Type I site-specific } \\
\text { restriction-modification system, } \\
\mathrm{R} \text { (restriction) subunit and } \\
\text { related helicases }\end{array}$ & 106 & 1 & 96 \\
\hline$x$ & 3654 & $\begin{array}{l}\text { Prophage maintenance system } \\
\text { killer protein }\end{array}$ & 1104 & 1 & 971 \\
\hline
\end{tabular}




\section{A.7 READ COVERAGE OF PAMC 27224 CONTIGS}

To assess variances in genome coverage, the sequencing reads of isolate PAMC 27224 were mapped back onto the 132 assembled contigs using bowtie2. Coverage of each individual contig was related to the median coverage (Figure A.9). A high ratio can indicate the presence of multiple copies of genes or features within that particular contig. While the method deployed here is less sophisticated than other described workflows (e.g. [344]), its purpose is only to serve as a rough estimate. Among the contigs with the highest coverage $(\approx 6$-fold higher than the median coverage) is the one containing the rRNA genes. This indicates that these are present in multiple copies throughout the genome, although they are annotated just once, likely due to the high fragmentation. Other contigs with high coverage usually contain one or more transposase-encoding genes, which could mean that their number, too, is underestimated from the annotated CDSs.

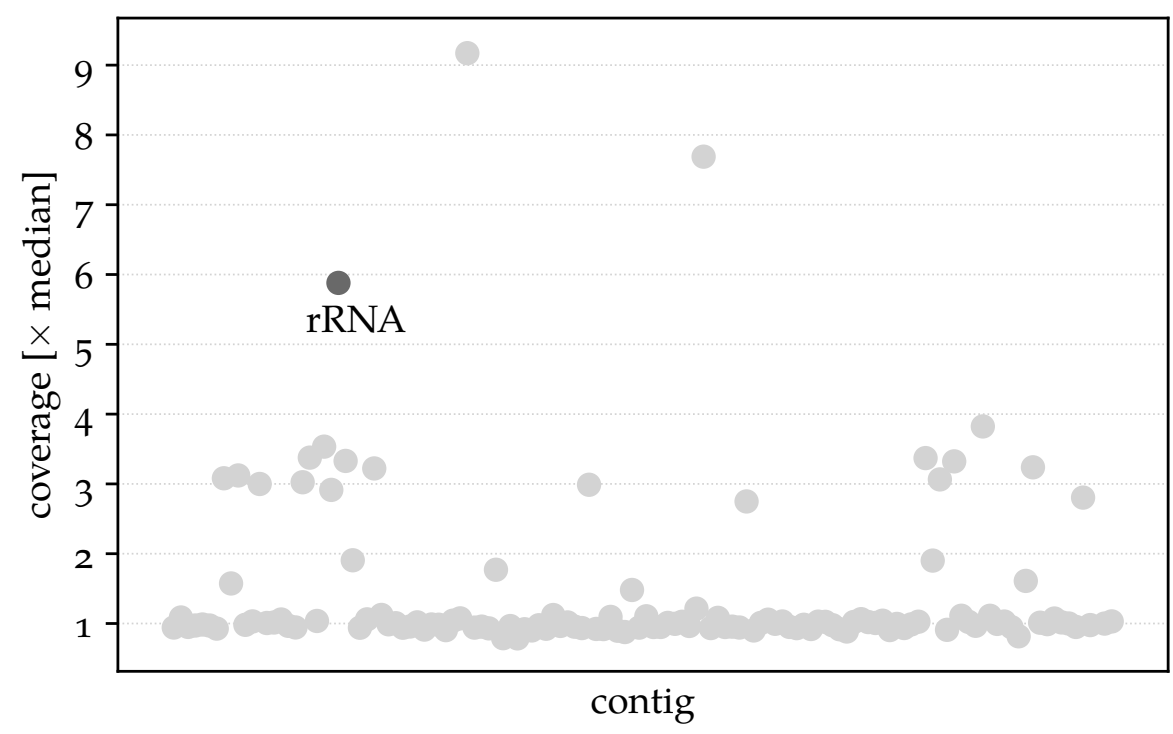

Figure A.9: Read coverage of isolate PAMC 27224 contigs. Reads were mapped back onto the genome using bowtie2, and the coverage for each contig was divided by the median coverage of all contigs. Contigs with a coverage of 3to 9 -fold the median exist, and usually encode one or multiple transposases. In addition, the contig carrying the rRNA genes shows a high coverage (highlighted in the plot), which indicates multiple copies of these genes in the genome. 

[1] Matthew A. Charette and Walter H.F. Smith. "The volume of earth's ocean." In: Oceanography 23.2 (June 2010), pp. 112-114. DOI: $10.5670 /$ oceanog. 2010.51.

[2] A. Baumgartner and E. Reichel. The world water balance: mean annual global, continental and maritime precipitation, evaporation and run-off. Elsevier Scientific Publishing Company, 1975. ISBN: 9780444998583 .

[3] Robert F. Adler et al. "The version-2 global precipitation climatology project (GPCP) monthly precipitation analysis (1979-present)." In: Journal of Hydrometeorology 4.6 (2003), pp. 1147-1167. DOI: 10.1175/1525-7541(2003)004<1147 : TVGPCP >2 . 0 . C0; 2.

[4] Paul Durack. "Ocean salinity and the global water cycle." In: Oceanography 28.1 (2015), pp. 20-31. DOI: 10.5670 / oceanog . 2015.03.

[5] J. I. Hedges and J. M. Oades. "Comparative organic geochemistries of soils and marine sediments." In: Organic Geochemistry 27.7-8 (1997), pp. 319-361. DOI: 10 . 1016 / S0146-6380 (97) 00056-9.

[6] Philippe Ciais et al. "Carbon and other biogeochemical cycles." In: Climate change 2013: The physical science basis. Contribution of Working Group I to the Fifth Assessment Report of the Intergovernmental Panel on Climate Change. Ed. by T.F. Stocker, D. Qin, G.-K. Plattner, M. Tignor, S.K. Allen, J. Boschung, A. Nauels, Y. Xia, V. Bex, and P.M. Midgley. Cambridge: Cambridge University Press, 2013. Chap. 6, pp. 465-570. ISBN: 978-1-107-66182-0. DOI: 10. $1017 /$ CB09781107415324.015.

[7] Christopher B Field, Michael J Behrenfeld, James T Randerson, and Paul Falkowski. "Primary production of the biosphere: integrating terrestrial and oceanic components." In: Science 281.5374 (1998), pp. 237-240. DOI: 10.1126/science.281.5374.237.

[8] Mary Ann Moran et al. "Deciphering ocean carbon in a changing world." In: Proceedings of the National Academy of Sciences 113.12 (2016), pp. 3143-3151. DOI: 10.1073/pnas. 1514645113.

[9] W B Whitman, D C Coleman, and W J Wiebe. "Prokaryotes: the unseen majority." In: Proceedings of the National Academy of Sciences of the United States of America 95.12 (1998), pp. 6578-83. DOI: $10.1073 /$ pnas. 95.12 .6578 .

[10] Marcel Margulies et al. "Genome sequencing in microfabricated high-density picolitre reactors." In: Nature $437 \cdot 7057$ (2005), pp. 376-380. DOI: 10.1038/nature03959. 
[11] Gerardo Turcatti, Anthony Romieu, Milan Fedurco, and Ana Paula Tairi. "A new class of cleavable fluorescent nucleotides: synthesis and optimization as reversible terminators for DNA sequencing by synthesis." In: Nucleic Acids Research 36.4 (2008). DOI: $10.1093 /$ nar/gkn021.

[12] Ângela M. Ribeiro, Andrew D. Foote, Anne Kupczok, Bárbara Frazão, Morten T. Limborg, Rosalía Piñeiro, Samuel Abalde, Sara Rocha, and Rute R. da Fonseca. "Marine genomics: News and views." In: Marine Genomics 31 (2017), pp. 1-8. DOI: https: //doi .org/10.1016/j . margen.2016.09.002.

[13] Sonja Voget et al. "Adaptation of an abundant Roseobacter RCA organism to pelagic systems revealed by genomic and transcriptomic analyses." In: The ISME Journal 9.2 (2015), pp. 371-384. DOI: 10.1038/ismej . 2014.134.

[14] Soo Je Park, Rohit Ghai, Ana Belén Martín-Cuadrado, Francisco Rodríguez-Valera, Won Hyong Chung, Kae Kyoung Kwon, Jung Hyun Lee, Eugene L. Madsen, and Sung Keun Rhee. "Genomes of two new ammonia-oxidizing archaea enriched from deep marine sediments." In: PLOS ONE 9.5 (2014), pp. 1-10. DOI: 10. 1371/journal . pone.0096449.

[15] B A Methé et al. "The psychrophilic lifestyle as revealed by the genome sequence of Colwellia psychrerythraea $34 \mathrm{H}$ through genomic and proteomic analyses." In: Proceedings of the National Academy of Sciences of the United States of America 102.31 (2005), pp. 10913-10918. DOI: Doi10.1073/Pnas.0504766102.

[16] Nicola Segata, Daniela Boernigen, Timothy L. Tickle, Xochitl C. Morgan, Wendy S. Garrett, and Curtis Huttenhower. "Computational meta'omics for microbial community studies." In: Molecular Systems Biology 9.1 (2013), pp. 1-15. DOI: 10.1038/ msb.2013.22.

[17] R. Chester and T.D. Jickells. Marine Geochemistry. Wiley, 2012. ISBN: 9781118349090.

[18] C. Wuchter et al. "Archaeal nitrification in the ocean." In: Proceedings of the National Academy of Sciences 103.33 (2006), pp. 1231712322. DOI: $10.1073 /$ pnas. 0600756103 .

[19] Brandon K Swan et al. "Potential for chemolithoautotrophy among ubiquitous bacteria lineages in the dark ocean." In: Science 333.September (2011), pp. 1296-9. DOI: 10.1126/science. 1203690.

[20] Mikhail V. Zubkov and Manuela Hartmann. "Ecological significance of microbial trophic mixing in the oligotrophic ocean: the Atlantic Ocean case studies." In: Microbial Ecology of the Oceans. Ed. by Josep M. Gasol and David L. Kirchman. 3. Hoboken, NJ: Wiley Blackwell, 2018. Chap. 3, pp. 99-122. ISBN: 9781119107194. 
[21] Author R a Duce et al. "Impacts of atmospheric Nitrogen on the anthropogenic open ocean." In: Science 320.5878 (2008), pp. 893897. DOI: 10.1126/science. 1150369.

[22] Jed A. Fuhrman and Åke Hagström. "Bacterial and archaeal community structure and its patterns." In: Microbial Ecology of the Oceans. Ed. by David L. Kirchman. 2. Hoboken, NJ: Wiley Blackwell, 2008. Chap. 3, pp. 45-90. ISBN: 978-o-470-04344-8.

[23] Stephen J. Giovannoni et al. "Genome streamlining in a cosmopolitan oceanic bacterium." In: Science 309.5738 (2005), pp. 12421245. DOI: 10.1126/science. 1114057.

[24] Alison Buchan, José M. González, and Mary Ann Moran. “Overview of the marine Roseobacter lineage." In: Applied and Environmental Microbiology 71.10 (2005), pp. 5665-5677. DOI: 10.1128/ AEM . 71. 10.5665-5677.2005.

[25] Josep M. Gasol and David L. Kirchman. "Introduction: The evolution of microbial ecology of the ocean." In: Microbial Ecology of the Oceans. Ed. by Josep M. Gasol and David L. Kirchman. 3. Hoboken, NJ: Wiley Blackwell, 2018. Chap. 1, pp. 1-46. ISBN: 9781119107194.

[26] Nicholas J. Russell. "Psychrophilic bacteria - Molecular adaptations of membrane lipids." In: Comparative Biochemistry and Physiology - A Physiology 118.3 (1997), pp. 489-493. DOI: 10.1016/ S0300-9629(97) 87354-9.

[27] Salvino D'Amico, Tony Collins, Jean-Claude Marx, Georges Feller, and Charles Gerday. "Psychrophilic microorganisms: Challenges for life." In: EMBO reports 7.4 (2006), pp. 385-9. DOI: $10.1038 / \mathrm{sj}$. embor .7400662 .

[28] Charles Gerday. "Psychrophily and catalysis." In: Biology (Basel) 2.2 (2013), pp. 719-741. DOI: 10.3390/biology2020719.

[29] Khawar Sohail Siddiqui and Ricardo Cavicchioli. "Cold-adapted enzymes." In: Annual Review of Biochemistry 75 (2006), pp. 403433. DOI: 10.1146/annurev . biochem.75.103004.142723.

[30] G. Horn, R. Hofweber, W. Kremer, and H. R. Kalbitzer. "Structure and function of bacterial cold shock proteins." In: Cellular and Molecular Life Sciences 64.12 (2007), pp. 1457-1470. DOI: $10.1007 / \mathrm{s} 00018-007-6388-4$.

[31] Christian Lorenz, Christina E. Lünse, and Mario Mörl. "tRNA modifications: Impact on structure and thermal adaptation." In: Biomolecules 7.2 (2017), p. 35. DOI: 10.3390/biom7020035.

[32] Naoki Sato, Takashi Tachikawa, Akira Wada, and Ayumi Tanaka. "Temperature-dependent regulation of the ribosomal small-subunit protein $\mathrm{S}_{21}$ in the cyanobacterium Anabaena variabilis M3." In: Journal of Bacteriology 179.22 (1997), pp. 7063-7071. DOI: 10. 1128/jb.179.22.7063-7071.1997. 
[33] Kevin P. O'Connell and Michael F. Thomashow. "Transcriptional organization and regulation of a polycistronic cold shock operon in Sinorhizobium meliloti RM1021 encoding homologs of the Escherichia coli major cold shock gene $\operatorname{csp} A$ and ribosomal protein gene rpsU." In: Applied and Environmental Microbiology 66.1 (2000), pp. 392-400. DOI: 10 . 1128/AEM . 66 . 1 . 392400.2000.

[34] Jason P. Chin et al. "Solutes determine the temperature windows for microbial survival and growth." In: Proceedings of the National Academy of Sciences of the United States of America 107.17 (2010), pp. 7835-7840. DOI: 10.1073/pnas. 1000557107.

[35] Corien Bakermans, Peter W. Bergholz, Debora F. Rodrigues, Tatiana A. Vishnivetskaya, Héctor L. Ayala-del Río, and James M. Tiedje. "Genomic and expression analyses of cold-adapted microorganisms." In: Polar Microbiology: Life in a Deep Freeze. Ed. by Robert V Miller and Lyle G Whyte. 1. ASM Press, 2012. Chap. 6, pp. 126-155. ISBN: 978-1-55581-604-9. DOI: 10.1128/ 9781555817183.

[36] S Venketesh and C Dayananda. "Properties, potentials, and prospects of antifreeze proteins." In: Critical Reviews in Biotechnology 28.1 (2008), pp. 57-82. DOI: 10 . 1080/07388550801891152.

[37] C. A. Mancuso Nichols, J. Guezennec, and J. P. Bowman. “Bacterial exopolysaccharides from extreme marine environments with special consideration of the Southern Ocean, sea ice, and deep-sea hydrothermal vents: A review." In: Marine Biotechnology 7.4 (2005), pp. 253-271. DOI: 10.1007/s10126-004-5118-2.

[38] C. Krembs, H. Eicken, K. Junge, and J. W. Deming. “High concentrations of exopolymeric substances in Arctic winter sea ice: Implications for the polar ocean carbon cycle and cryoprotection of Diatoms." In: Deep-Sea Research Part I: Oceanographic Research Papers 49.12 (2002), pp. 2163-2181. DOI: 10.1016/S09670637(02)00122-X.

[39] Claudine Médigue et al. "Coping with cold: The genome of the versatile marine Antarctica bacterium Pseudoalteromonas haloplanktis TAC125." In: Genome Research 15.10 (2005), pp. 13251335. DOI: $10.1101 / \mathrm{gr} .4126905$.

[40] Jacqueline Goordial et al. “Cold adaptive traits revealed by comparative genomic analysis of the eurypsychrophile Rhodococcus sp. JG3 isolated from high elevation McMurdo Dry Valley permafrost, Antarctica." In: FEMS Microbiology Ecology 92.2 (2016), pp. 1-11. DOI: 10.1093/femsec/fiv154.

[41] Kevin R Arrigo. "Sea ice ecosystems." In: Annu. Rev. Marine. Sci 6 (2014), pp. 439-467. DOI: 10 .1146/annurev-marine-010213135103. 
[42] Antje Boetius, Alexandre M Anesio, Jody W Deming, Jill Mikucki, and Josephine Z Rapp. "Microbial ecology of the cryosphere: sea ice and glacial habitats." In: Nature Reviews Microbiology 13.11 (2014), pp. 677-690. DOI: 10.1038/nrmicro3522.

[43] Christopher Krembs, Hajo Eicken, and Jody W Deming. "Exopolymer alteration of physical properties of sea ice and implications for ice habitability and biogeochemistry in a warmer Arctic." In: Proceedings of the National Academy of Sciences 108.9 (2011), pp. 3653-3658. DoI: 10.1073/pnas. 1100701108.

[44] Marcela Ewert and Jody Deming. "Sea ice microorganisms: Environmental constraints and extracellular responses." In: Biology (Basel) 2.2 (2013), pp. 603-628. DOI: 10. 3390/biology2020603.

[45] David N. Thomas, Stathys Papadimitriou, and Christine Michel. "Biogeochemistry of sea ice." In: Sea Ice. Ed. by David N. Thomas and Gerhard S. Diekmann. 2. Wiley-Blackwell, 2010. Chap. 12, pp. 425-467. ISBN: 978-1-4051-8580-6.

[46] R. Bargagli. "Environmental contamination in Antarctic ecosystems." In: Science of the Total Environment 400.1-3 (2008), pp. 212226. DOI: $10.1016 / \mathrm{j}$. scitotenv . 2008.06.062.

[47] K. Van de Velde, P. Vallelonga, J. P. Candelone, K. J.R. Rosman, V. Gaspari, G. Cozzi, C. Barbante, R. Udisti, P. Cescon, and C. $\mathrm{F}$. Boutron. "Pb isotope record over one century in snow from Victoria Land, Antarctica." In: Earth and Planetary Science Letters 232.1-2 (2005), pp. 95-108. DOI: 10.1016/j . epsl . 2005 .01.007.

[48] Ralf Ebinghaus, Hans H. Kock, Christian Temme, Jürgen W. Einax, Astrid G. Löwe, Andreas Richter, John P. Burrows, and William H. Schroeder. "Antarctic springtime depletion of atmospheric mercury." In: Environmental Science and Technology 36.6 (2002), pp. 1238-1244. DOI: 10.1021/es015710z.

[49] Evert Nieboer and David H S Richardson. "The replacement of the nondescript term 'heavy metals' by a biologically and chemically significant classification of metal ions." In: Environmental Pollution (Series B) 1 (1980), pp. 3-26.

[50] S Madronich, R L McKenzie, L O Björn, and M M Caldwell. "Changes in biologically active ultraviolet radiation reaching the Earth's surface." In: Journal of photochemistry and photobiology. B: Biology 46.1-3 (1998), pp. 5-19. DOI: 10.1039/b700017k.

[51] Robin Brinkmeyer, Katrin Knittel, Jutta Jürgens, Horst Weyland, Rudolf Amann, and Elisabeth Helmke. "Diversity and structure of bacterial communities in Arctic versus Antarctic pack ice." In: Applied and Environmental Microbiology 69.11 (2003), pp. 66106619. DOI: 10.1128/AEM. 69.11.6610-6619.2003. 
[52] Jeff S Bowman, Simon Rasmussen, Nikolaj Blom, Jody W Deming, Søren Rysgaard, and Thomas Sicheritz-Ponten. "Microbial community structure of Arctic multiyear sea ice and surface seawater by 454 sequencing of the $16 \mathrm{~S}$ RNA gene." In: The ISME journal 6.1 (2012), pp. 11-20. DOI: 10.1038/ismej .2011.76.

[53] Rebecca O M Cowie, Gareth J. Williams, Elizabeth W. Maas, K. Matt Voyles, and Ken G. Ryan. "Antarctic sea-ice microbial communities show distinct patterns of zonation in response to algal-derived substrates." In: Aquatic Microbial Ecology 73.2 (2014), pp. 123-134. DOI: 10.3354/ame01710.

[54] M. Hünken, J. Harder, and G. O. Kirst. “Epiphytic bacteria on the Antarctic ice diatom Amphiprora kufferathii Manguin cleave hydrogen peroxide produced during algal photosynthesis." In: Plant Biology 10.4 (2008), pp. 519-526. DOI: $10.1111 / \mathrm{j} .1438-$ $8677.2008 .00040 . x$.

[55] Stefanie VanTrappen, Joris Mergaert, and Jean Swings. "Loktanella salsilacus gen. nov., sp. nov., Loktanella fryxellensis sp. nov. and Loktanella vestfoldensis sp. nov., new members of the Rhodobacter group isolated from microbial mats in Antarctic lakes." In: International Journal of Systematic and Evolutionary Microbiology 54.4 (2004), pp. 1263-1269. DOI: 10.1099/ijs.0.03006-0.

[56] De Chao Zhang, Hui Rong Li, Yu Hua Xin, Hong Can Liu, Zhen Ming Chi, Pei Jin Zhou, and Yong Yu. "Phaeobacter arcticus sp. nov., a psychrophilic bacterium isolated from the Arctic." In: International Journal of Systematic and Evolutionary Microbiology 58.6 (2008), pp. 1384-1387. Dor: 10.1099/ijs.0.65708-0.

[57] Tsuneo Shiba. "Roseobacter litoralis gen. nov., sp. nov., and Roseobacter denitrificans sp. nov., aerobic pink-pigmented bacteria which contain bacteriochlorophyll a." In: Systematic and Applied Microbiology 14.2 (1991), pp. 140-145. DOI: $10.1016 /$ S0723 2020 (11) 80292- 4 .

[58] Keizo Shimada. "Aerobic anoxygenic phototrophs." In: Anoxygenic Photosynthetic Bacteria. Ed. by Robert E. Blankenship, Michael T. Madigan, and Carl E. Bauer. Dordrecht: Kluwer Academic Publishers, 1995. Chap. 6, pp. 105-122. ISBN: 0-7923-3681-X.

[59] Meinhard Simon, Carmen Scheuner, Jan P. Meier-Kolthoff, Thorsten Brinkhoff, Irene Wagner-Döbler, Marcus Ulbrich, HansPeter Klenk, Dietmar Schomburg, Jörn Petersen, and Markus Göker. "Phylogenomics of Rhodobacteraceae reveals evolutionary adaptation to marine and non-marine habitats." In: The ISME Journal 11.6 (2017), pp. 1483-1499. ISSN: 1751-7362. DOI: 10.1038/ismej . 2016.198.

[6o] Ryan J Newton et al. "Genome characteristics of a generalist marine bacterial lineage." In: The ISME journal 4.6 (2010), pp. 784798. DOI: $10.1038 /$ ismej . 2009.150. 
[61] John Vollmers. "Molekularbiologische Charakterisierung und vergleichende Genomik von ausgewählten Vertretern mariner Roseobacter-Stämme." dissertation. Georg-August Universität Göttingen, 2013. URL: \url\{https : / / ediss . uni-goettingen . de / handle / 11858 / 00 - 1735 - 0000 - 0022 - 5E61 - 6 ? locale attribute=en\}.

[62] Haiwei Luo and Mary Ann Moran. "Evolutionary ecology of the marine Roseobacter clade." In: Microbiology and Molecular Biology Reviews 78.4 (2014), pp. 573-587. DOI: 10 . 1128 / MMBR . 00020-14.

[63] Ying Sun, Kate E. Powell, Way Sung, Michael Lynch, Mary Ann Moran, and Haiwei Luo. "Spontaneous mutations of a model heterotrophic marine bacterium." In: ISME Journal 11.7 (2017), pp. 1713-1718. DOI: 10.1038/ismej . 2017.20.

[64] Hui Wang, Jürgen Tomasch, Michael Jarek, and Irene WagnerDöbler. "A dual-species co-cultivation system to study the interactions between Roseobacters and dinoflagellates." In: Frontiers in Microbiology 5.JUN (2014), p. 311. DOI: 10. 3389/fmicb. 2014. 00311.

[65] Bryndan P. Durham et al. "Cryptic carbon and sulfur cycling between surface ocean plankton." In: Proceedings of the National Academy of Sciences 112.2 (2015), pp. 453-457. DOI: 10.1073 / pnas. 1413137112 .

[66] Elke Allers, Laura Gómez-Consarnau, Jarone Pinhassi, Josep M. Gasol, Karel Šimek, and Jakob Pernthaler. "Response of Alteromonadaceae and Rhodobacteriaceae to glucose and phosphorus manipulation in marine mesocosms." In: Environmental Microbiology 9.10 (2007), pp. 2417-2429. DOI: 10 . $1111 /$ j . $1462-$ $2920.2007 .01360 . x$.

[67] Jose MGonzalez, Ronald P Kiene, and Mary Ann Moran. “Transformation of sulfur compounds by an abundant lineage of marine bacteria in the $\alpha$-subclass of the class Proteobacteria." In: Applied and Environmental Microbiology 65.9 (1999), pp. 38103819 .

[68] Jingli Liu, Ji Liu, Sheng-Hui Zhang, Jinchang Liang, Heyu Lin, Delei Song, Gui-Peng Yang, Jonathan D. Todd, and Xiao-Hua Zhang. "Novel insights into bacterial dimethylsulfoniopropionate catabolism in the East China Sea." In: Frontiers in Microbiology 9.December (2018), p. 3206. DOI: 10. 3389/fmicb. 2018. 03206.

[69] Martin F. Polz, Dana E. Hunt, Sarah P. Preheim, and Daniel M. Weinreich. "Patterns and mechanisms of genetic and phenotypic differentiation in marine microbes." In: Philosophical Transactions of the Royal Society B: Biological Sciences 361.1475 (2006), pp. 2009-2021. DOI: 10.1098/rstb.2006. 1928. 
[70] M. A. Moran et al. "Ecological genomics of marine roseobacters." In: Applied and Environmental Microbiology 73.14 (2007), pp. 4559-4569. DOI: 10.1128/AEM. 02580-06.

[71] Marc Solioz and Barry Marrs. "The gene transfer agent of Rhodopseudomonas capsulata: Purification and characterization of its nucleic acid." In: Archives of Biochemistry and Biophysics 181 (1977), pp. 300-307. DOI: 10. 1016/0003-9861 (77) 90508-2.

[72] Erin J. Biers, Kui Wang, Catherine Pennington, Robert Belas, Feng Chen, and Mary Ann Moran. "Occurrence and expression of gene transfer agent genes in marine bacterioplankton." In: Applied and Environmental Microbiology 74.10 (2008), pp. 29332939. DOI: 10.1128/AEM. 02129-07.

[73] Catalina Maria Alejandro-Marín, Rafael Bosch, and Balbina Nogales. "Comparative genomics of the protocatechuate branch of the $\beta$-ketoadipate pathway in the Roseobacter lineage." In: Marine Genomics 17 (2014), pp. 25-33. DOI: 10 . 1016/ j . margen . 2014.05 .008$.

[74] Pascal Bartling, John Vollmers, and Jörn Petersen. "The first world swimming championships of roseobacters--Phylogenomic insights into an exceptional motility phenotype." In: Systematic and Applied Microbiology 41.6 (2018), pp. 544-554. DOI: 10.1016/j . syapm.2018.08.012.

[75] Michal Koblížek, Qiang Zheng, Aleš Horák, and Miroslav Oborník. "Regressive evolution of photosynthesis in the Roseobacter clade." In: Advances in Botanical Research Volume 66 - Genome Evolution of Photosynthetic Bacteria. Ed. by Jean-pierre Jacquot, Pierre Gadal, and J. Thomas Beatty. Amsterdam: Elsevier Ltd., 2013. Chap. 13, pp. 386-405. ISBN: 9780123979230. DOI: 10. 1016/B978-0-12397923-0.00012-6.

[76] J.J. Gosink, R.P. Herwig, and J.T. Staley. “Octadecabacter arcticus gen. nov., sp. nov., and O. antarcticus, sp. nov., nonpigmented, psychrophilic gas vacuolate bacteria from polar sea ice and water." In: Systematic and Applied Microbiology 20.3 (1997), pp. 356365. DOI: 10 . 1016/S0723-2020(97) 80003-3.

[77] John Vollmers, Sonja Voget, Sascha Dietrich, Kathleen Gollnow, Maike Smits, Katja Meyer, Thorsten Brinkhoff, Meinhard Simon, and Rolf Daniel. "Poles apart: Arctic and Antarctic Octadecabacter strains share high genome plasticity and a new type of xanthorhodopsin." In: PloS one 8.5 (2013), e63422. DOI: 10.1371/journal. pone. 0063422 .

[78] Sooyeon Park and Jung Hoon Yoon. “Octadecabacter jejudonensis sp. nov., isolated from the junction between the ocean and a freshwater spring and emended description of the genus Octadecabacter." In: International Journal of Systematic and Evolutionary Microbiology 64.PART 3 (2014), pp. 719-724. DOI: 10.1099/ ijs.0.057513-0. 
[79] Sara Billerbeck, Julia Orchard, Brian J. Tindall, Helge Ansgar Giebel, Thorsten Brinkhoff, and Meinhard Simon. "Description of Octadecabacter temperatus sp. nov., isolated from the southern North Sea, emended descriptions of the genus Octadecabacter and its species and reclassification of Octadecabacter jejudonensis Park and Yoon 2014 as Pseudooctadecabacter jejudonensis gen. nov comb. nov." In: International Journal of Systematic and Evolutionary Microbiology 65.6 (2015), pp. 1967-1974. DOI: 10.1099/ijs . 0.000205 .

[80] Yung Mi Lee, You-jung Jung, Soon Gyu Hong, Ji Hee Kim, and Hong Kum Lee. "Diversity and physiological characteristics of culturable bacteria from marine sediments of Ross Sea, Antarctica." In: Korean Journal of Microbiology 50.2 (2014), pp. 119-127.

[81] Young Ok Kim, In Suk Park, Sooyeon Park, Bo Hye Nam, Ji Min Park, Dong Gyun Kim, and Jung Hoon Yoon. "Octadecabacter ascidiaceicola sp. nov., isolated from a sea squirt (Halocynthia roretzi)." In: International Journal of Systematic and Evolutionary Microbiology 66.1 (2016), pp. 296-301. DOI: 10.1099/ijsem. 0. 000715.

[82] Sooyeon Park, Sun Young Yoon, Yong-taek Jung, and Jung-hoon Yoon. "Octadecabacter ponticola sp. nov., isolated from seawater." In: International Journal of Systematic and Evolutionary Microbiology 66.10 (2016), pp. 4179-4184. DOI: 10.1099/i j sem. 0. 001332.

[83] Sven Breider, Silke Pradella, Carmen Scheuner, Jörn Petersen, Peter Schumann, Hans-Peter Klenk, Thorsten Brinkhoff, Markus Göker, and Anne Fiebig. "Genome-scale data suggest reclassifications in the Leisingera-Phaeobacter cluster including proposals for Sedimentitalea gen. nov. and Pseudophaeobacter gen. nov." In: Frontiers in Microbiology 5.August (2014), pp. 1-13. DOI: 10.3389/fmicb.2014.00416.

[84] Marike Palmer, Emma T. Steenkamp, Martin P. A. Coetzee, Jochen Blom, and Stephanus N. Venter. "Genome-based characterization of biological processes that differentiate closely related bacteria." In: Frontiers in Microbiology 9.February (2018), pp. 1-23. DOI: 10.3389/fmicb. 2018.00113.

[85] Ramon Rosselló-Móra and Rudolf Amann. "Past and future species definitions for bacteria and archaea." In: Systematic and Applied Microbiology 38.4 (2015), pp. 209-216. Dor: 10.1016/ j . syapm.2015.02.001.

[86] Qi Long Qin, Bin Bin Xie, Xi Ying Zhang, Xiu Lan Chen, Bai Cheng Zhou, Jizhong Zhou, Aharon Oren, and Yu Zhong Zhang. "A proposed genus boundary for the prokaryotes based on genomic insights." In: Journal of Bacteriology 196.12 (2014), pp. 22102215. DOI: $10.1128 / \mathrm{JB}$. 01688-14. 
[87] Pd Cantino and Kevin De Queiroz. International code of phylogenetic nomenclature, Version 4c. 2010. URL: https: //www . ohio. edu/phylocode/PhyloCode4c . pdf $\{\backslash \%\}$ Ahttp : //www . ohio . edu/PhyloCode/PhyloCode4c.pdf.

[88] Mark W. Williams, Sharon E. Stammerjohn, Hugh W. Ducklow, Andrew G. Fountain, Edward A. G. Schuur, and John L. Campbell. "The disappearing cryosphere: Impacts and ecosystem responses to rapid cryosphere loss." In: BioScience 62.4 (2012), pp. 405-415. DOI: 10.1525/bio.2012.62.4.11.

[89] Natascha Selje, Meinhard Simon, and Thorsten Brinkhoff. "A newly discovered Roseobacter cluster in temperate and polar oceans." In: Nature 427.6973 (2004), pp. 445-448. DOI: 10.1038/ nature02272.

[90] Eileen Y. Koh, William Phua, and Ken G. Ryan. "Aerobic anoxygenic phototrophic bacteria in Antarctic sea ice and seawater." In: Environmental Microbiology Reports 3.6 (2011), pp. 710-716. DOI: $10.1111 / \mathrm{j} .1758-2229.2011 .00286 . \mathrm{x}$.

[91] Frédéric Delsuc, Henner Brinkmann, and Hervé Philippe. "Phylogenomics and the reconstruction of the tree of life." In: Nature Reviews Genetics 6.5 (2005), pp. 361-375. DOI: 10. 1038/nrg1603.

[92] Miklós Csurös and István Miklós. "Streamlining and large ancestral genomes in archaea inferred with a phylogenetic birthand-death model." In: Molecular Biology and Evolution 26.9 (2009), pp. 2087-2095. DOI: 10.1093/molbev/msp123.

[93] Haiwei Luo, Miklós Csũros, Austin L Hughes, and Mary Ann Moran. "Evolution of divergent life history strategies in marine Alphaproteobacteria." In: mBio 4.4 (2013), eoo373-13. DOI: 10. 1128/mBio.00373-13.Editor.

[94] Riccardo Percudani, Davide Carnevali, and Vincenzo Puggioni. “Ureidoglycolate hydrolase, amidohydrolase, lyase: How errors in biological databases are incorporated in scientific papers and vice versa." In: Database 2013 (2013), bato71. DOI: 10 . 1093 / database/bat071.

[95] Antoine Danchin. "Coping with inevitable accidents in metabolism." In: Microbial Biotechnology 10.1 (2017), pp. 57-72. DOI: 10.1111/1751-7915.12461.

[96] Antoine Danchin and Gang Fang. "Unknown unknowns: essential genes in quest for function." In: Microbial Biotechnology 9.5 (2016), pp. 530-540. DOI: 10.1111/1751-7915.12384.

[97] Anthony M. Bolger, Marc Lohse, and Bjoern Usadel. “Trimmomatic: A flexible trimmer for Illumina sequence data." In: Bioinformatics 30.15(2014), pp. 2114-2120. DOI: 10.1093/bioinformatics/ btu170. 
[98] Anton Bankevich et al. "SPAdes: A new genome assembly algorithm and its applications to single-cell sequencing." In: Journal of Computational Biology 19.5 (2012), pp. 455-477. DOI: 10.1089/ cmb. 2012.0021.

[99] Torsten Seemann. "Prokka: Rapid prokaryotic genome annotation." In: Bioinformatics 30.14 (2014), pp. 2068-2069. DOI: 10. 1093/bioinformatics/btu153.

[100] Andreas Leimbach. bac-genomics-scripts: Bovine E. coli mastitis comparative genomics edition. 2016. DOI: 10. 5281/zenodo. 215824.

[101] Donovan H Parks, Michael Imelfort, Connor T Skennerton, Philip Hugenholtz, and Gene W Tyson. "CheckM: assessing the quality of microbial genomes recovered from isolates, single cells, and metagenomes." In: Genome Research 25 (2015), pp. 1043-1055. DOI: 10.1101/gr. 186072.114.

[102] Minoru Kanehisa, Miho Furumichi, Mao Tanabe, Yoko Sato, and Kanae Morishima. "KEGG: New perspectives on genomes, pathways, diseases and drugs." In: Nucleic Acids Research 45.D1 (2017), pp. D353-D361. Dor: 10. 1093/nar/gkw1092.

[103] Jan P. Meier-Kolthoff, Alexander F. Auch, Hans Peter Klenk, and Markus Göker. "Genome sequence-based species delimitation with confidence intervals and improved distance functions." In: BMC Bioinformatics 14 (2013). DOI: 10.1186/1471-2105-14-60.

[104] Katherine J. Boettcher, Bruce J. Barber, and John T. Singer. "Use of antibacterial agents to elucidate the etiology of juvenile oyster disease (JOD) in Crassostrea virginica and numerical dominance of an $\alpha$-Proteobacterium in JOD-affected animals." In: Applied and Environmental Microbiology 65.6 (1999), pp. 2534-2539.

[105] Qiliang Lai, Junwei Cao, Jun Yuan, Fuying Li, and Zongze Shao. "Celeribacter indicus sp. nov., a polycyclic aromatic hydrocarbondegrading bacterium from deep-sea sediment and reclassification of Huaishuia halophila as Celeribacter halophilus comb. nov." In: International Journal of Systematic and Evolutionary Microbiology 64.2014 (2014), pp. 4160-4167. DOI: 10. 1099/i js.0.069039Q.

[106] L. Y. Suarez-Suarez, I. Brunet-Galmes, J. M. Piña Villalonga, J. A. Christie-Oleza, A. Peña, A. Bennasar, J. Armengaud, B. Nogales, and R. Bosch. "Draft genome sequence of Citreicella aestuarii strain 357, a member of the Roseobacter clade isolated without xenobiotic pressure from a petroleum-polluted beach." In: Journal of Bacteriology 194.19 (2012), pp. 5464-5465. DOI: 10 . 1128/JB. 01261-12.

[107] Hanno Biebl, Martin Allgaier, Brian J. Tindall, Michal Kobližek, Heinrich Lünsdorf, Rüdiger Pukall, and Irene Wagner-Döbler. "Dinoroseobacter shibae gen. nov., sp. nov., a new aerobic phototrophic bacterium isolated from dinoflagellates." In: Interna- 
tional Journal of Systematic and Evolutionary Microbiology 55.3 (2005), pp. 1089-1096. DOI: 10.1099/ijs.0.63511-0.

[108] Sooyeon Park and Jung Hoon Yoon. "Jannaschia aquimarina sp. nov., isolated from seawater." In: International Journal of Systematic and Evolutionary Microbiology 62.11 (2012), pp. 2631-2636. DOI: 10.1099/ijs.0.038448-0.

[109] M. C. Macián, D. R. Arahal, E. Garay, W. Ludwig, K. H. Schleifer, and Maria J. Pujalte. "Jannaschia rubra sp. nov., a red-pigmented bacterium isolated from sea water." In: International Journal of Systematic and Evolutionary Microbiology 55.2 (2005), pp. 649-653. DOI: 10.1099/ijs.0.63412-0.

[110] Liming Liu, Yang Li, Jing Zhang, Zhemin Zhou, Jie Liu, Xiaomin Li, Jingwen Zhou, Guocheng Du, Lei Wang, and Jian Chen. "Complete genome sequence of the industrial strain Ketogulonicigenium vulgare WSH-o01." In: Journal of Bacteriology 193.21 (2011), pp. 6108-6109. DOI: 10.1128/JB.06007-11.

[111] Xiang Hua Xiong et al. "Complete genome sequence of the bacterium Ketogulonicigenium vulgare Y25." In: Journal of Bacteriology 193.1 (2011), pp. 315-316. DOI: 10.1128/JB.01189-10.

[112] Sooyeon Park, Yong Taek Jung, Su Jung Choi, and Jung Hoon Yoon. "Phaeobacter aquaemixtae sp. nov., isolated from the junction between the ocean and a freshwater spring." In: International Journal of Systematic and Evolutionary Microbiology 67.9 (2017), pp. 3446-3451. DOI: 10.1099/ijsem.0.002136.

[113] Ilse Vandecandelaere, Eveline Segaert, Alfonso Mollica, Marco Faimali, and Peter Vandamme. "Leisingera aquimarina sp. nov., isolated from a marine electroactive biofilm, and emended descriptions of Leisingera methylohalidivorans Schaefer et al. 2002, Phaeobacter daeponensis Yoon et al. 2007 and Phaeobacter inhibens Martens et al. 2006." In: International Journal of Systematic and Evolutionary Microbiology 58.12 (2008), pp. 2788-2793. DOI: 10. 1099/ijs. 0.65844-0.

[114] Ilse Vandecandelaere, Eveline Segaert, Alfonso Mollica, Marco Faimali, and Peter Vandamme. "Phaeobacter caeruleus sp. nov., a blue-coloured, colony-forming bacterium isolated from a marine electroactive biofilm." In: International Journal of Systematic and Evolutionary Microbiology 59.5 (2009), pp. 1209-1214. DOI: 10.1099/ijs.0.002642-0.

[115] Jung Hoon Yoon, So Jung Kang, Soo Young Lee, and Tae Kwang Oh. "Phaeobacter daeponensis sp. nov., isolated from a tidal flat of the Yellow Sea in Korea." In: International Journal of Systematic and Evolutionary Microbiology 57.4 (2007), pp. 856-861. DOI: 10. 1099/ijs.0.64779-0. 
[116] Jk Schaefer, Kd Goodwin, Ir McDonald, J Colin Murrell, and Rs Oremland. "Leisingera methylohalidivorans gen. nov., sp. nov., a marine methylotroph that grows on methyl bromide." In: International journal of systematic and evolutionary microbiology 52.3 (2002), pp. 851-9. DOI: 10.1099/ijs.0.01960-0. The.

[117] Andrew J. Collins and Spencer V. Nyholm. "Draft genome of Phaeobacter gallaeciensis ANG1, a dominant member of the accessory nidamental gland of Euprymna scolopes." In: Journal of Bacteriology 193.13 (2011), pp. 3397-3398. DOI: 10.1128/JB.0513911.

[118] Lyudmila A. Romanenko, Naoto Tanaka, Galina M. Frolova, Vassilii I. Svetashev, and Valery V. Mikhailov. "Litoreibacter albidus gen. nov., sp. nov. and Litoreibacter janthinus sp. nov., members of the class Alphaproteobacteria isolated from the seashore." In: International Journal of Systematic and Evolutionary Microbiology 61.1 (2011), pp. 148-154. DOI: 10.1099/ijs. 0. Q19513-0.

[119] Byung Yong Kim, Hang Yeon Weon, Jung A. Son, Chang Muk Lee, Seung Beom Hong, Young Ah Jeon, Bon Sung Koo, and Soon Wo Kwon. "Thalassobacter arenae sp. nov., isolated from sea sand in Korea." In: International Journal of Systematic and Evolutionary Microbiology 59.3 (2009), pp.487-490. DOI: 10. 1099/ ijs.0.65841-0.

[120] Young Ok Kim, In Suk Park, Sooyeon Park, Bo Hye Nam, Ji Min Park, Dong Gyun Kim, and Jung Hoon Yoon. "Litoreibacter ascidiaceicola sp. nov., isolated from the golden sea squirt Halocynthia aurantium." In: International Journal of Systematic and Evolutionary Microbiology 66.3 (2016), pp. 1174-1179. DOI: 10. 1099/ijsem.0.000849.

[121] Shoichi Hosoya and Akira Yokota. "Loktanella atrilutea sp. nov., isolated from seawater in Japan." In: International Journal of Systematic and Evolutionary Microbiology 57.9 (2007), pp. 1966-1969. DOI: $10.1099 / \mathrm{ijs}$.0.64978-0.

[122] Taishi Tsubouchi, Yasuhiro Shimane, Kozue Mori, Masayuki Miyazaki, Akihiro Tame, Katsuyuki Uematsu, Tadashi Maruyama, and Yuji Hatada. "Loktanella cinnabarina sp. nov., isolated from a deep subseafloor sediment, and emended description of the genus Loktanella." In: International Journal of Systematic and Evolutionary Microbiology 63.PART4 (2013), pp. 1390-1395. DOI: 10.1099/ijs.0.043174-0.

[123] Stanley C K Lau, Mandy M Y Tsoi, Xiancui Li, Ioulia Plakhotnikova, Madeline Wu, Po Keung Wong, and Pei Yuan Qian. "Loktanella hongkongensis sp. nov., a novel member of the $\alpha$ Proteobacteria originating from marine biofilms in Hong Kong waters." In: International Journal of Systematic and Evolutionary Microbiology 54.6 (2004), pp. 2281-2284. DOI: 10.1099/ijs. 0. 63294-0. 
[124] Hang Yeon Weon, Byung Yong Kim, Seung Hee Yoo, Jong Shik Kim, Soon Wo Kwon, Seung Joo Go, and Erko Stackebrandt. "Loktanella koreensis sp. nov., isolated from sea sand in Korea." In: International Journal of Systematic and Evolutionary Microbiology 56.9 (2006), pp. 2199-2202. DOI: 10.1099/i js. 0.64276-0.

[125] Jung Hoon Yoon, Yong Taek Jung, and Jung Sook Lee. “Loktanella litorea sp. nov., isolated from seawater." In: International Journal of Systematic and Evolutionary Microbiology 63.1 (2013), pp. 175-18o. DOI: 10.1099/ijs.0.039198-0.

[126] Young Gun Moon, Seong Hae Seo, Soon Dong Lee, and Moon Soo Heo. "Loktanella pyoseonensis sp. nov., isolated from beach sand, and emended description of the genus Loktanella." In: International Journal of Systematic and Evolutionary Microbiology 60.4 (2010), pp. 785-789. DOI: 10.1099/ijs.0.011072-0.

[127] Elena P. Ivanova, Natalia V. Zhukova, Anatoly M. Lysenko, Nataliya M. Gorshkova, Alexander F. Sergeev, Valery V. Mikhailov, and John P. Bowman. "Loktanella agnita sp. nov. and Loktanella rosea sp. nov., from the north-west Pacific Ocean." In: International Journal of Systematic and Evolutionary Microbiology 55.5 (2005), pp. 2203-2207. DOI: 10.1099/ijs.0.63461-0.

[128] J. Liang, Z. Zhang, Y. Liu, M. Wang, and X.-H. Zhang. “Loktanella sediminum sp. nov., isolated from marine surface sediment." In: International Journal of Systematic and Evolutionary Microbiology 65.Pt 2 (2015), pp. 686-691. DoI: 10. 1099/i js . 0.070680-0.

[129] Soon Dong Lee. "Loktanella tamlensis sp. nov., isolated from seawater." In: International Journal of Systematic and Evolutionary Microbiology 62.PART 3 (2012), pp. 586-590. DOI: 10.1099/ijs. 0.029462-0.

[130] David H. Green, Lyndon E. Llewellyn, Andrew P. Negri, Susan I. Blackburn, and Christopher J.S. Bolch. "Phylogenetic and functional diversity of the cultivable bacterial community associated with the paralytic shellfish poisoning dinoflagellate Gymnodinium catenatum." In: FEMS Microbiology Ecology 47.3 (2004), pp. 345-357. DOI: 10.1016/S0168-6496(03)00298-8.

[131] Kiyoung Lee, Yoe Jin Choo, Stephen J. Giovannoni, and Jang Cheon Cho. "Maritimibacter alkaliphilus gen. nov., sp. nov., a genome-sequenced marine bacterium of the Roseobacter clade in the order Rhodobacterales." In: International Journal of Systematic and Evolutionary Microbiology 57.7 (2007), pp. 1653-1658. DOI: 10.1099/ijs. 0.64960-0.

[132] Ilse Vandecandelaere, Olivier Nercessian, Eveline Segaert, Wafa Achouak, Alfonso Mollica, Marco Faimali, and Peter Vandamme. "Nautella italica gen. nov., sp. nov., isolated from a marine electroactive biofilm." In: International Journal of Systematic and Evolutionary Microbiology 59.4 (2009), pp. 811-817. DOI: 10.1099/ ijs.0.002683-0. 
[133] Maria J. Pujalte, M. C. Macián, D. R. Arahal, W. Ludwig, K. H. Schleifer, and E. Garay. "Nereida ignava gen. nov., sp. nov., a novel aerobic marine $\alpha$-Proteobacterium that is closely related to uncultured Prionitis (alga) gall symbionts." In: International Journal of Systematic and Evolutionary Microbiology 55.2 (2005), pp. 631-636. DOI: 10.1099/ijs.0.63442-0.

[134] Jang Cheon Cho and Stephen J. Giovannoni. “Oceanicola granulosus gen. nov., sp. nov. and Oceanicola batsensis sp. nov., poly- $\beta-$ hydroxybutyrate-producing marine bacteria in the order 'Rhodobacterales'." In: International Journal of Systematic and Evolutionary Microbiology 54.4 (2004), pp. 1129-1136. DOI: 10.1099/ijs . Q.03015-0.

[135] Jun Gu, Bin Guo, Ya-Nan Wang, Su-Lin Yu, Ryuhei Inamori, $\mathrm{Ri} \mathrm{Qu}, \mathrm{Yu}-\mathrm{Guang} \mathrm{Ye}$, and Xiao-Lei Wu. "Oceanicola nanhaiensis sp. nov., isolated from sediments of the South China Sea." In: International journal of systematic and evolutionary microbiology 57.2007 (2007), pp. 157-160. DOI: 10.1099/i js . 0.64532-0.

[136] Tony Gutierrez, William B Whitman, Marcel Huntemann, Alex Copeland, Amy Chen, Neha Vargese, Nikos C Kyrpides, Manoj Pillay, and Natalia Ivanova. "Genome sequence of Oceanicola sp. Strain MCTG156(1a), isolated from a Scottish coastal phytoplankton net sample." In: Genome Announcements 5.32 (2017), pp. 9-10. DOI: 10.1128/genomeA .00796-17.

[137] Young Kyung Kwon, Jennifer Jooyoun Kim, Ji Hyung Kim, Seon Mi Jeon, Bo Ram Ye, Jiyi Jang, Soo Jin Heo, Se Chang Park, Do Hyung Kang, and Chulhong Oh. "Draft genome sequence of the xylan-degrading marine bacterium strain S124, representing a novel species of the genus Oceanicola." In: Journal of Bacteriology 194.22 (2012), p. 6325. DOI: 10.1128/JB.01614-12.

[138] Keshao Liu, Rui Zong, Qipei Li, Yingnan Fu, Yongle Xu, Yanan Wang, and Nianzhi Jiao. "Oceaniovalibus guishaninsula gen. nov., sp. nov., a marine bacterium of the family Rhodobacteraceae." In: Current Microbiology 64.4 (2012), pp. 385-391. DOI: 10. 1007 / s00284-012-0081-z.

[139] Jang Cheon Cho and Stephen J. Giovannoni. "Parvularcula bermudensis gen. nov., sp. nov., a marine bacterium that forms a deep branch in the $\alpha$-Proteobacteria." In: International Journal of Systematic and Evolutionary Microbiology 53.4 (2003), pp. 1031-1036. DOI: $10.1099 / \mathrm{ijs} .0 .02566-0$.

[140] Jang Cheon Cho and Stephen J. Giovannoni. "Pelagibaca bermudensis gen. nov., sp. nov., a novel marine bacterium within the Roseobacter clade in the order Rhodobacterales." In: International Journal of Systematic and Evolutionary Microbiology 56.4 (2006), pp. 855-859. DOI: 10.1099/ijs.0.64063-0. 
[141] C Ruiz-Ponte, V Cilia, C Lambert, and J L Nicolas. "Roseobacter gallaeciensis sp. nov., a new marine bacterium isolated from rearings and collectors of the scallop Pecten maximus." In: International Journal of Systematic Bacteriology 48 (1998), pp. 537542.

[142] Torben Martens, Thorsten Heidorn, Rüdiger Pukal, Meinhard Simon, Brian J. Tindall, and Thorsten Brinkhoff. "Reclassification of Roseobacter gallaeciensis Ruiz-Ponte et al. 1998 as Phaeobacter gallaeciensis gen. nov., comb. nov., description of Phaeobacter inhibens sp. nov., reclassification of Ruegeria algicola (Lafay et al. 1995) Uchino et al. 1999 as Marinovum algicola gen. nov., comb. nov., and emended descriptions of the genera Roseobacter, Ruegeria and Leisingera." In: International Journal of Systematic and Evolutionary Microbiology 56.6 (2006), pp. 1293-1304. DOI: 10.1099/ijs.0.63724-0.

[143] Nora Buddruhs, Silke Pradella, Markus Goker, Orsola Pauker, Rüdiger Pukall, Cathrin Sproer, Peter Schumann, Jörn Petersen, and Thorsten Brinkhoff. "Molecular and phenotypic analyses reveal the non-identity of the Phaeobacter gallaeciensis type strain deposits CIP 105210T and DSM 17395." In: International Journal of Systematic and Evolutionary Microbiology 63.PART 11 (2013), pp. 4340-4349. DOI: 10.1099/ijs.0.053900-0.

[144] Helge Ansgar Giebel et al. "Planktomarina temperata gen. nov., sp. nov., belonging to the globally distributed RCA cluster of the marine Roseobacter clade, isolated from the German Wadden Sea." In: International Journal of Systematic and Evolutionary Microbiology 63.PART 11 (2013), pp. 4207-4217. DOI: 10 . 1099/ ijs.0.053249-0.

[145] Ilnam Kang, Kevin L. Vergin, Hyun Myung Oh, Ahyoung Choi, Stephen J. Giovannoni, and Jang Cheon Cho. "Genome sequence of strain HTCC2083, a novel member of the marine clade Roseobacter." In: Journal of Bacteriology 193.1 (2011), pp. 319-320. DOI: $10.1128 / \mathrm{JB} .01268-10$.

[146] Ilnam Kang, Hyun Myung Oh, Kevin L. Vergin, Stephen J. Giovannoni, and Jang Cheon Cho. "Genome sequence of the marine al phaproteobacterium HTCC2150, assigned to the Roseobacter clade." In: Journal of Bacteriology 192.23 (2010), pp. 6315-6316. DOI: $10.1128 / \mathrm{JB} .01088-10$.

[147] Colleen M Hansel and Chris A Francis. "Coupled photochemical and enzymatic Mn(II) oxidation pathways of a planktonic Roseobacter-like bacterium." In: Applied and Environmental Microbiology 72.Ii (2006), pp. 3543-3549. DOI: 10.1128/AEM. 72 . 5. 3543.

[148] Guizhen Li, Qiliang Lai, Chunming Dong, Rui Ma, Yaping Du, Xiupian Liu, Fengqin Sun, Zongze Shao, and Peisheng Yan. "Roseovarius atlanticus sp. nov., isolated from surface seawater." 
In: International Journal of Systematic and Evolutionary Microbiology 66.2 (2016), pp. 639-644. DOI: 10.1099/i jsem. 0.000768.

[149] Hanno Biebl, Martin Allgaier, Heinrich Lünsdorf, Rüdiger Pukall, Brian J. Tindall, and Irene Wagner-Döbler. "Roseovarius mucosus sp. nov., a member of the Roseobacter clade with trace amounts of bacteriochlorophyll a." In: International Journal of Systematic and Evolutionary Microbiology 55.6 (2005), pp. 23772383. DOI: $10.1099 / \mathrm{ijs} .0 .63832-0$.

[150] José M. González et al. "Silicibacter pomeroyi sp. nov. and Roseovarius nubinhibens sp. nov., dimethylsulfoniopropionate-demethylating bacteria from marine environments." In: International Journal of Systematic and Evolutionary Microbiology 53.5 (2003), pp. 1261-1269. DOI: 10.1099/ijs.0.02491-0.

[151] Hendrik Schäfer, Ian R. McDonald, Phil D. Nightingale, and J. Colin Murrell. "Evidence for the presence of a CmuA methyltransferase pathway in novel marine methyl halide-oxidizing bacteria." In: Environmental Microbiology 7.6 (2005), pp. 839-852. DOI: $10.1111 / \mathrm{j} .1462-2920.2005 .00757 . \mathrm{x}$.

[152] Tony Gutierrez et al. "Genome sequence of Roseovarius sp. strain MCTG156(2b) isolated from a phytoplankton net trawl on the Scottish West Coast." In: Genome Announcements 5.35 (2017), pp. 1-2.

[153] Hans-Jürgen Rüger and Manfred G Höfle. “Marine star-shapedaggregate-forming bacteria : Agrobacterium atlanticum sp. nov.; Agrobacterium meteori sp. nov.; Agrobacterium ferrugineum sp. nov., nom. rev.; Agrobacterium gelatinovorum sp. nov., nom. rev.; and Agrobacterium stellulatum sp. nov., nom. rev." In: International Journal of Systematic Bacteriology 42.1 (1992), pp. 133-143. DOI: $10.1099 / 00207713-42-1-133$.

[154] Jina Lee et al. "Ruegeria conchae sp. nov., isolated from the ark clam Scapharca broughtonii." In: International Journal of Systematic and Evolutionary Microbiology 62.12 (2012), pp. 2851-2857. DOI: 10.1099/ijs.0.037283-0.

[155] Margot Doberva, Sophie Sanchez-Ferandin, Yoan Ferandin, Laurent Intertaglia, Julie Croué, Marcelino Suzuki, Philippe Lebaron, and Raphaël Lami. "Genome sequence of the sponge-associated Ruegeria halocynthiae strain MOLA R1/13b, a marine Roseobacter with two quorum-sensing-based communication systems." In: Genome announcements 2.5 (2014), p. 4389. DOI: 10.1128 / genomeA.00998- 14.

[156] Eva C. Sonnenschein et al. "Global occurrence and heterogeneity of the Roseobacter-clade species Ruegeria mobilis." In: ISME Journal 11.2 (2017), pp. 569-583. DOI: 10.1038/i smej . 2016.111. 
[157] J M Gonzalez, F Mayer, M A Moran, and R E Hodson. "Sagittula stellata gen. nov., sp. nov., a lignin-transforming bacterium from a coastal environment." In: International Journal of Systematic Bacteriology 47.3 (1997), pp. 773-780. DOI: 10 . 1099/0020771347-3-773.

[158] Fengqin Sun, Baojiang Wang, Xiupian Liu, Qiliang Lai, Yaping Du, Guangyu Li, Jie Luo, and Zongze Shao. "Leisingera nanhaiensis sp. nov., isolated from marine sediment." In: International Journal of Systematic and Evolutionary Microbiology 60.2 (2010), pp. 275-280. DOI: 10.1099/ijs.0.010439-0.

[159] Dong H. Choi and Byung C. Cho. "Shimia marina gen. nov., sp. nov., a novel bacterium of the Roseobacter clade isolated from biofilm in a coastal fish farm." In: International Journal of Systematic and Evolutionary Microbiology 56.8 (2006), pp. 18691873. DOI: 10.1099/ijs.0.64235-0.

[160] Saranya Kanukollu et al. “Genome sequence of Shimia str. SKo13, a representative of the Roseobacter group isolated from marine sediment." In: Standards in Genomic Sciences 11.1 (2016). DOI: 10.1186/s40793-016-0143-0.

[161] Jung Hoon Yoon, So Jung Kang, Mi Hwa Lee, and Tae Kwang Oh. "Description of Sulfitobacter donghicola sp. nov., isolated from seawater of the East Sea in Korea, transfer of Staleya guttiformis Labrenz et al. 2000 to the genus Sulfitobacter as Sulfitobacter guttiformis comb. nov. and emended description of the genus Sulfitobacter." In: International Journal of Systematic and Evolutionary Microbiology 57.8 (2007), pp. 1788-1792. DOI: 10.1099/ijs.0.65071-0.

[162] Min Jung Kwak, Jung Sook Lee, Keun Chul Lee, Kwang Kyu Kim, Mi Kyung Eom, Byung Kwon Kim, and Jihyun F. Kim. "Sulfitobacter geojensis sp. nov., Sulfitobacter noctilucae sp. nov., and Sulfitobacter noctilucicola sp. nov., isolated from coastal seawater." In: International Journal of Systematic and Evolutionary Microbiology 64.2014 (2014), pp. 3760-3767. DOI: 10.1099/ijs . 0.065961-0.

[163] Matthias Labrenz, B. J. Tindall, Paul A. Lawson, Matthew D. Collins, Peter Schumann, and Peter Hirsch. "Staleya guttiformis gen. nov., sp. nov. and Sulfitobacter brevis sp. nov., $\alpha-3$-Proteobacteria from hypersaline, heliothermal and meromictic antarctic Ekho Lake." In: International Journal of Systematic and Evolutionary Microbiology 50.1 (2000), pp. 303-313. DOI: 10.1099/ 00207713-50-1-303.

[164] Rüdiger Pukall, Daniela Buntefuß, Anja Frühling, Manfred Rohde, Reiner M Kroppenstedt, Jutta Burghardt, Philippe Lebar, Laetitia Bernard, and Erko Stackebrandtl. "Sulfitobacter mediterraneus sp. nov., a new sulfite-oxidizing member of the $\alpha$-Proteobacteria." In: International Journal of Systematic Bacteriology 49.2 (1999), pp. 513-519. DOI: 10.1099/00207713-49-2-513. 
[165] Maria Mas-lladó, Joana Maria Pi na-villalonga, Isabel Brunetgalmés, and Balbina Nogales. "Draft genome sequences of two isolates of the Roseobacter group, Sulfitobacter sp. Strains 3 SOLIMARog and 1FIGIMARog, from harbors of Mallorca Island (Mediterranean Sea)." In: Genome Announcements 2.3 (2014), eoo350-14. DOI: 10.1128/genomeA .00350-14. Copyright.

[166] Zhuan Hong, Qiliang Lai, Qiaoqi Luo, Simeng Jiang, Ruilin Zhu, Junrong Liang, and Yahui Gao. "Sulfitobacter pseudonitzschiae sp. nov., isolated from the toxic marine diatom Pseudo-nitzschia multiseries." In: International Journal of Systematic and Evolutionary Microbiology 65.1 (2015), pp. 95-100. DOI: 10.1099 / i js . 0 . Q64972-0.

[167] N. Y. D. Ankrah, T. Lane, C. R. Budinoff, M. K. Hadden, and A. Buchan. "Draft genome sequence of Sulfitobacter sp. CB2047, a member of the Roseobacter clade of marine bacteria, isolated from an Emiliania huxleyi bloom." In: Genome Announcements 2.6 (2014), e01125-14-eo1125-14. DOI: 10 . 1128/genomeA . 0112514.

[168] Andrew J. Collins, Matthew S. Fullmer, Johann P. Gogarten, and Spencer V. Nyholm. "Comparative genomics of Roseobacter clade bacteria isolated from the accessory nidamental gland of Euprymna scolopes." In: Frontiers in Microbiology 6.FEB (2015), pp. 1-14. DOI: 10.3389/fmicb. 2015.00123.

[169] M.C. Macián, D. R. Arahal, E. Garay, W. Ludwig, K. H. Schleifer, and Maria J. Pujalte. "Thalassobacter stenotrophicus gen. nov., sp. nov., a novel marine $\alpha$-Proteobacterium isolated from Mediterranean sea water." In: International Journal of Systematic and Evolutionary Microbiology 55.1 (2005), pp. 105-110. DOI: 10.1099/ ijs.0.63275-0.

[170] Jiao Yan Ying, Bao Jun Wang, Xin Dai, Su Sheng Yang, Shuang Jiang Liu, and Zhi Pei Liu. "Wenxinia marina gen. nov., sp. nov., a novel member of the Roseobacter clade isolated from oilfield sediments of the South China Sea." In: International Journal of Systematic and Evolutionary Microbiology 57.8 (2007), pp. 17111716. DOI: 10.1099/ijs.0.64825-0.

[171] Marcus Lechner, Sven Findeiß, Lydia Steiner, Manja Marz, Peter F Stadler, and Sonja J Prohaska. "Proteinortho : detection of (co)orthologs in large-scale analysis." In: BMC Bioinformatics 12.1 (2011), p. 124. DOI: 10.1186/1471-2105-12-124.

[172] Robert C. Edgar. "MUSCLE: Multiple sequence alignment with high accuracy and high throughput." In: Nucleic Acids Research 32.5 (2004), pp. 1792-1797. DOI: 10.1093/nar/gkh340.

[173] Alexandros Stamatakis. "RAxML version 8: A tool for phylogenetic analysis and post-analysis of large phylogenies." In: Bioinformatics 30.9 (2014), pp.1312-1313. DOI: 10.1093/bioinformatics/ btu033. 
[174] Leonidas Salichos, Alexandros Stamatakis, and Antonis Rokas. "Novel information theory-based measures for quantifying incongruence among phylogenetic trees." In: Molecular Biology and Evolution 31.5 (2014), pp. 1261-1271. DOI: 10.1093/molbev/ msu061.

[175] F. Pedregosa et al. "Scikit-learn: machine learning in Python." In: Journal of Machine Learning Research 12 (2011), pp. 2825-2830.

[176] Eric Jones, Travis Oliphant, Pearu Peterson, et al. SciPy: Open source scientific tools for Python. [Online; accessed <today>]. 2001. URL: http://www . scipy . org/.

[177] Jaime Huerta-Cepas, François Serra, and Peer Bork. "ETE 3: Reconstruction, analysis, and visualization of phylogenomic data." In: Molecular Biology and Evolution 33.6 (2016), pp. 16351638. DOI: $10.1093 / \mathrm{molbev} / \mathrm{msw} 046$.

[178] Daniel H. Huson and Celine Scornavacca. "Dendroscope 3: An interactive tool for rooted phylogenetic trees and networks." In: Systematic Biology 61.6 (2012), pp. 1061-1067. DOI: 10.1093/ sysbio/sys062.

[179] Natalia N Ivanova, Konstantinos Mavromatis, I-min a Chen, Victor M Markowitz, and Nikos C Kyrpides. Standard operating procedure for the annotations of genomes and metagenomes submitted to the Integrated Microbial Genomes Expert Review (IMG-ER) system. 2007. URL: http: //imgweb.jgi-psf.org/w/doc/img_ er_ann.pdf.

[180] Michael Y. Galperin, Kira S. Makarova, Yuri I. Wolf, and Eugene V. Koonin. "Expanded microbial genome coverage and improved protein family annotation in the COG database." In: Nucleic Acids Research 43.D1 (2015), pp. D261-D269. DOI: 10 . 1093/nar/gku1223.

[181] Miklós Csurös. "Count: Evolutionary analysis of phylogenetic profiles with parsimony and likelihood." In: Bioinformatics 26.15 (2010), pp. 1910-1912. DOI: 10.1093/bioinformatics/btq315.

[182] Alexey Drozdetskiy, Christian Cole, James Procter, and Geoffrey J. Barton. "JPred4: A protein secondary structure prediction server." In: Nucleic Acids Research 43.W1 (2015), W389-W394. DOI: $10.1093 /$ nar/gkv332.

[183] Yoav Benjamini and Yosef Hochberg. "Controlling the false discovery rate: a practical and powerful approach to multiple testing." In: Journal of the Royal Statistical Society. Series B (Methodological) 57.1 (1995), pp. 289-300. DOI: 10.2307/2346101.

[184] Folker Meyer, D Paarmann, M D'Souza, and Etal. “The metagenomics RAST server-a public resource for the automatic phylogenetic and functional analysis of metagenomes." In: BMC bioinformatics 9 (2008), p. 386. DOI: 10. 1186/1471-2105-9-386. 
[185] Karin Lagesen, Peter Hallin, Einar Andreas Rødland, Hans Henrik Stærfeldt, Torbjørn Rognes, and David W. Ussery. "RNAmmer: Consistent and rapid annotation of ribosomal RNA genes." In: Nucleic Acids Research 35.9 (2007), pp. 3100-3108. DOI: 10 . 1093/nar/gkm160.

[186] Ben Langmead and Steven L. Salzberg. "Fast gapped-read alignment with Bowtie 2." In: Nature Methods 9.4 (2012), pp. 357-359. DOI: $10.1038 /$ nmeth. 1923.

[187] Leighton Pritchard, Rachel H. Glover, Sonia Humphris, John G. Elphinstone, and Ian K. Toth. "Genomics and taxonomy in diagnostics for food security: Soft-rotting enterobacterial plant pathogens." In: Analytical Methods 8.1 (2016), pp. 12-24. DOI: $10.1039 / \mathrm{c} 5 \mathrm{ay} 02550 \mathrm{~h}$.

[188] George Vernikos, Duccio Medini, David R. Riley, and Hervé Tettelin. "Ten years of pan-genome analyses." In: Current Opinion in Microbiology 23 (2015), pp. 148-154. DOI: 10. 1016/j . mib. 2014.11 .016$.

[189] Eugene V. Koonin and Yuri I. Wolf. "Genomics of bacteria and archaea: The emerging dynamic view of the prokaryotic world." In: Nucleic Acids Research 36.21 (2008), pp. 6688-6719. DOI: 10 . 1093/nar/gkn668.

[190] R. Overbeek, M. Fonstein, M. D'Souza, G. D. Pusch, and N. Maltsev. "The use of gene clusters to infer functional coupling." In: Proceedings of the National Academy of Sciences 96.6 (1999), pp. 2896-2901. DOI: 10.1073/pnas.96.6.2896.

[191] Inoka C. Perera and Anne Grove. "Molecular mechanisms of ligand-mediated attenuation of DNA binding by MarR family transcriptional regulators." In: Journal of Molecular Cell Biology 2.5 (2010), pp. 243-254. DOI: 10. 1093/jmcb/mjq021.

[192] Ziyang Hao et al. "The multiple antibiotic resistance regulator MarR is a copper sensor in Escherichia coli." In: Nature Chemical Biology 10.1 (2014), pp. 21-28. DOI: 10. 1038/nchembio. 1380.

[193] Steven P. Wilkinson and Anne Grove. "Ligand-responsive transcriptional regulation by members of the MarR family of winged helix proteins." In: Current Issues in Molecular Biology 8.1 (2006), pp. 51-62.

[194] Béatrice Py et al. "Molecular organization, biochemical function, cellular role and evolution of NfuA, an atypical Fe-S carrier." In: Molecular Microbiology 86.1 (2012), pp. 155-171. DOI: 10.1111/j.1365-2958.2012.08181.x.

[195] Kai Zhao, Mingzhu Liu, and Richard R. Burgess. "The global transcriptional response of Escherichia coli to induced $\sigma_{32}$ protein involves $\sigma 32$ regulon activation followed by inactivation and degradation of $\sigma_{32}$ in vivo." In: Journal of Biological Chemistry 280.18 (2005), pp. 17758-17768. DOI: 10.1074/jbc. M500393200. 
[196] Phyllis I. Hanson and Sidney W. Whiteheart. "AAA+ proteins: Have engine, will work." In: Nature Reviews Molecular Cell Biology 6.7 (2005), pp. 519-529. DOI: 10.1038/nrm1684.

[197] Ryuta Tobe and Hisaaki Mihara. "Delivery of selenium to selenophosphate synthetase for selenoprotein biosynthesis." In: Biochimica et Biophysica Acta - General Subjects 1862.11 (2018), pp. 2433-2440. DOI: 10.1016/j . bbagen. 2018.05.023.

[198] Agathe Tarze, Marc Dauplais, Ioana Grigoras, Myriam Lazard, Nguyet Thanh Ha-Duong, Frédérique Barbier, Sylvain Blanquet, and Pierre Plateau. "Extracellular production of hydrogen selenide accounts for thiol-assisted toxicity of selenite against Saccharomyces cerevisiae." In: Journal of Biological Chemistry 282.12 (2007), pp. 8759-8767. DOI: 10.1074/jbc. M610078200.

[199] Y. Ogasawara, G. M. Lacourciere, K. Ishii, and T. C. Stadtman. "Characterization of potential selenium-binding proteins in the selenophosphate synthetase system." In: Proceedings of the $\mathrm{Na}$ tional Academy of Sciences 102.4 (2005), pp. 1012-1016. DOI: 10. $1073 /$ pnas . 0409042102 .

[200] Obed Béjà et al. "Bacterial rhodopsin: Evidence for a new type of phototrophy in the sea." In: Science 289.5486 (2000), pp. 19021906. DOI: $10.1126 /$ science. 289.5486. 1902.

[201] Omri M Finkel, Oded Béjà, and Shimshon Belkin. "Global abundance of microbial rhodopsins." In: The ISME journal 7.2 (2013), pp. 448-51. DOI: 10.1038/ismej . 2012.112.

[202] Jed a Fuhrman, Michael S Schwalbach, and Ulrich Stingl. "Proteorhodopsins: An array of physiological roles?" In: Nature reviews. Microbiology 6.6 (2008), pp. 488-94. DOI: $10.1038 /$ nrmicro1893.

[203] Sergei P Balashov, Eleonora S Imasheva, Vladimir a Boichenko, Josefa Antón, Jennifer M Wang, and Janos K Lanyi. "Xanthorhodopsin: a proton pump with a light-harvesting carotenoid antenna." In: Science 309.5743 (2005), pp. 2061-4. DOI: 10. 1126/ science. 1118046.

[204] J. M. Walter, D. Greenfield, C. Bustamante, and J. Liphardt. "Light-powering Escherichia coli with proteorhodopsin." In: Proceedings of the National Academy of Sciences 104.7 (2007), pp. 24082412. DOI: $10.1073 /$ pnas. 0611035104.

[205] Laura Gómez-Consarnau, Neelam Akram, Kristoffer Lindell, Anders Pedersen, Richard Neutze, Debra L. Milton, José M. González, and Jarone Pinhassi. "Proteorhodopsin phototrophy promotes survival of marine bacteria during starvation." In: PLoS Biology 8.4 (2010), pp. 2-11. DOI: 10.1371/journal . pbio. 1000358.

[206] Michael J Weickert and Sankar Adhya. "A family of bacterial regulators homologous to Gal and Lac repressors." In: Journal of Biological Chemistry 267.22 (1992), pp. 15869-15874. 
[207] Erwin Schneider. "ABC transporters catalyzing carbohydrate uptake." In: Research in Microbiology 152.3-4 (2001), pp. 303-310. DOI: $10.1016 /$ S0923-2508(01)01201-3.

[208] E C C Lin. "Glycerol dissimilation and its regulation in bacteria." In: Annual Review of Microbiology 30.1 (1976), pp. 535-578. DOI: 10.1146/annurev.mi .30.100176.002535.

[209] Rahel Elevi Bardavid and Aharon Oren. "Dihydroxyacetone metabolism in Salinibacter ruber and in Haloquadratum walsbyi." In: Extremophiles 12.1 (2008), pp. 125-131. DOI: 10.1007/s00792Q07-0114-x.

[210] K I Sørensen and B Hove-Jensen. "Ribose catabolism of Escherichia coli: characterization of the $r p i B$ gene encoding ribose phosphate isomerase B and of the rpiR gene, which is involved in regulation of $r p i B$ expression." In: Journal of bacteriology 178.4 (1996), pp. 1003-11. DOI: 10.1016/j . jfca.2014.04.004.

[211] Yefei Zhu, Renu Nandakumar, Marat R. Sadykov, Nandakumar Madayiputhiya, Thanh T. Luong, Rosmarie Gaupp, Chia Y. Lee, and Greg A. Somerville. "RpiR homologues may link Staphylococcus aureus RNAIII synthesis and pentose phosphate pathway regulation." In: Journal of Bacteriology 193.22 (2011), pp. 6187-6196. DOI: 10.1128/JB.05930-11.

[212] Abdelali Daddaoua, Tino Krell, and Juan Luis Ramos. "Regulation of glucose metabolism in Pseudomonas. The phosphorylative branch and Entner-Doudoroff enzymes are regulated by a repressor containing a sugar isomerase domain." In: Journal of Biological Chemistry 284.32 (2009), pp. 21360-21368. DOI: 10.1074/jbc.M109.014555.

[213] Christopher Mulligan, Marcus Fischer, and Gavin H. Thomas. "Tripartite ATP-independent periplasmic (TRAP) transporters in bacteria and archaea." In: FEMS Microbiology Reviews 35.1 (2011), pp. 68-86. DOI: 10.1111/j.1574-6976.2010.00236.x.

[214] Gavin H. Thomas, Thomas Southworth, Maria Rocio LeónKempis, and Andrew Leech. "Novel ligands for the extracellular solute receptors of two bacterial TRAP transporters." In: Microbiology 152.1 (2006), pp. 187-198. DOI: 10. 1099/mic. 0. 28334-0.

[215] Shin Kurihara, Shinpei Oda, Kenji Kato, Hyeon Guk Kim, Takashi Koyanagi, Hidehiko Kumagai, and Hideyuki Suzuki. "A novel putrescine utilization pathway involves $\gamma$-glutamylated intermediates of Escherichia coli K-12." In: Journal of Biological Chemistry 28o.6 (2005), pp. 4602-4608. DOI: 10. 1074/jbc. M411114200.

[216] H. J. Rhee, Eui Jin Kim, and J. K. Lee. "Physiological polyamines: Simple primordial stress molecules." In: Journal of Cellular and Molecular Medicine 11.4 (2007), pp. 685-703. DoI: $10.1111 / \mathrm{j}$. $1582-4934.2007 .00077 . x$. 
[217] John A. Leigh and Jeremy A. Dodsworth. "Nitrogen regulation in bacteria and archaea." In: Annual Review of Microbiology 61.1 (2007), pp. 349-377. DOI: 10.1146/annurev . micro.61.080706. 093409.

[218] Juan L Ramos, Manuel Martínez-Bueno, Antonio J MolinaHenares, Wilson Terán, Kazuya Watanabe, Xiaodong Zhang, María Trinidad Gallegos, Richard Brennan, and Raquel Tobes. "The TetR family of transcriptional repressors." In: Microbiology and Molecular Biology Reviews 69.2 (2005), pp. 326-356. DOI: 10.1128/MMBR . 69.2.326-356.2005.

[219] Tatiana Claro da Silva, James E Polli, and Peter W Swaan. "The solute carrier family 10 (SLC10): beyond bile acid transport." In: Molecular Aspects of Medicine 34.2-3 (2013), pp. 252-269. DOI: $10.1016 / \mathrm{j}$. mam. 2012.07 .004 .

[220] Nien Jen Hu, So Iwata, Alexander D. Cameron, and David Drew. "Crystal structure of a bacterial homologue of the bile acid sodium symporter ASBT." In: Nature 478.7369 (2011), pp. 408411. DOI: $10.1038 /$ nature 10450 .

[221] Bodo Philipp. "Bacterial degradation of bile salts." In: Applied Microbiology and Biotechnology 89.4 (2011), pp. 903-915. DOI: 10. $1007 / s 00253-010-2998-0$.

[222] W. A. Anderson and Boris Magasanik. "The pathway of myoinositol degradation in Aerobacter aerogenes: Identification of the intermediate 2-deoxy-5-keto-D-gluconic acid." In: Biological Chemistry 246.18 (1971), pp. 5658-5661.

[223] W A Anderson and Boris Magasanik. "The pathway of myoinositol degradation in Aerobacter aerogenes: Conversion of 2deoxy-5-keto-D-gluconic acid to glycolytic intermediates." In: Biological Chemistry 246.18 (1971), pp. 5662-5675.

[224] Thomas Berman and Boris Magasanik. "The pathway of myoinositol degradation in Aerobacter aerogenes: Dehydrogenation and dehydration." In: Biological Chemistry 241.4 (1966), pp. 800806 .

[225] Thomas Berman and Boris Magasanik. "The pathway of myoinositol degradation in Aerobacter aerogenes: Ring scission." In: Biological Chemistry 241.4 (1966), pp. 807-813.

[226] Ken Ichi Yoshida, Masanori Yamaguchi, Tetsuro Morinaga, Masaki Kinehara, Maya Ikeuchi, Hitoshi Ashida, and Yasutaro Fujita. "myo-inositol catabolism in Bacillus subtilis." In: Journal of Biological Chemistry 283.16 (2008), pp. 10415-10424. DOI: 10.1074/ jbc. M708043200.

[227] Mohammad Ali Ghalambor and Edward C Heath. "The metabolism of L-Fucose." In: Journal of Biological Chemistry 237.8 (1962), pp. 2423-2427. 
[228] Myron F. Goodman and Roger Woodgate. "Translesion DNA polymerases." In: Cold Spring Harbor Perspectives in Biology 5.10 (2013), pp. 1-20. DOI: 10.1101/cshperspect.a010363.

[229] Charles R Booth and Sasha Madronich. "Radiation amplification factors: Improved formulation accounts for large increases in ultraviolet radiation associated with Antarctic ozone depletion." In: Ultraviolet Radiation in Antarctica: Measurements and Biological Effects. Ed. by Susan C Weiler and Polly A Penhale. Washington, D.C.: American Geophysical Union, 1994. Chap. 3, pp. 39-42. ISBN: 9780875908410. DOI: 10.1029/AR062.

[230] B. Yeiser, E. D. Pepper, M. F. Goodman, and S. E. Finkel. “SOSinduced DNA polymerases enhance long-term survival and evolutionary fitness." In: Proceedings of the National Academy of Sciences 99.13 (2002), pp. 8737-8741. DOI: 10.1073 / pnas . Q92269199.

[231] Zijun Xiao and Ping Xu. "Acetoin metabolism in bacteria." In: Critical Reviews in Microbiology 33.2 (2007), pp. 127-140. DOI: 10. $1080 / 10408410701364604$.

[232] David White, James Drummond, and Clay Fuqua. The physiology and biochemistry of prokaryotes. 4. New York: Oxford University Press, 2012. ISBN: 978-0-19-539304-0.

[233] E Almaas, B Kovács, T Vicsek, ZN Oltvai, and A-L Barabási. "Global organization of metabolic fluxes in the bacterium Escherichia coli." In: Nature 427.6977 (2004), pp. 839-43. DOI: 10. 1038/nature02289.

[234] Sébastien Zappa, Keran Li, and Carl E Bauer. "The tetrapyrrole biosynthetic pathway and its regulation in Rhodobacter capsulatus." In: Recent Advances in Phototrophic Prokaryotes. Ed. by Patrick C Hallenbeck. 1. Springer, 2010. Chap. 13, pp. 229-250. ISBN: 978-1-4419-1527-6. DOI: 10 . 1007/978-1-4419-1528-3.

[235] H. Mihara and N. Esaki. "Bacterial cysteine desulfurases: Their function and mechanisms." In: Applied Microbiology and Biotechnology 60.1-2 (2002), pp. 12-23. DOI: 10.1007 / s00253-002 1107-4.

[236] Ron Ortenberg and Jon Beckwith. "Functions of thiol-disulfide oxidoreductases in E. coli: Redox myths, realities, and practicalities." In: Antioxidants \& Redox Signaling 5.4 (2003), pp. 403-411. DOI: $10.1089 / 152308603768295140$.

[237] Krzysztof Ginalski, Lisa Kinch, Leszek Rychlewski, and Nick V. Grishin. "DCC proteins: a novel family of thiol-disulfide oxidoreductases." In: Trends in Biochemical Sciences 29.7 (2004), pp. 339-342. DOI: 10.1016/j .tibs. 2004.05 .002$. 
[238] Eric Eichhorn, Jan R. Van Der Ploeg, Michael A. Kertesz, and Thomas Leisinger. "Characterization of $\alpha$-ketoglutarate-dependent taurine dioxygenase from Escherichia coli." In: Journal of Biological Chemistry 272.37 (1997), pp. 23031-23036. DOI: 10.1074/ jbc.272.37.23031.

[239] L. Pollegioni, L. Piubelli, S. Sacchi, M. S. Pilone, and G. Molla. "Physiological functions of D-amino acid oxidases: From yeast to humans." In: Cellular and Molecular Life Sciences 64.11 (2007), pp. 1373-1394. DOI: 10.1007/s00018-007-6558-4.

[240] Rungaroon Waditee, Takashi Hibino, Yoshito Tanaka, Tatsunosuke Nakamura, Aran Incharoensakdi, and Teruhiro Takabe. "Halotolerant Cyanobacterium Aphanothece halophytica contains an $\mathrm{Na}^{+} / \mathrm{H}^{+}$antiporter, homologous to eukaryotic ones, with novel ion specificity affected by C-terminal tail." In: The Journal of Biological Chemistry 276.40 (2001), pp. 36931-36938. DOI: 10. $1074 / \mathrm{jbc} . \mathrm{M} 103650200$.

[241] Renate Dippel and Winfried Boos. "The maltodextrin system of Escherichia coli: Metabolism and transport." In: Journal of Bacteriology 187.24 (2005), pp. 8322-8331. DOI: 10.1128/JB . 187. 24. 8322-8331.2005.

[242] Sungmin Hwang, Kyoung Hwa Choi, Jieun Kim, and Jaeho Cha. "Biochemical characterization of 4 - $\alpha$-glucanotransferase from Saccharophagus degradans 2-40 and its potential role in glycogen degradation." In: FEMS Microbiology Letters 344.2 (2013), pp. 145-151. DOI: 10.1111/1574-6968.12167.

[243] H. M. Lam and M. E. Winkler. "Characterization of the complex pdxH-tyrS operon of Escherichia coli K-12 and pleiotropic phenotypes caused by pdxH insertion mutations." In: Journal of Bacteriology 174.19 (1992), pp. 6033-6045. DOI: 10.1128/jb. 174 . 19.6033-6045.1992.

[244] Kristian Kvint, Laurence Nachin, Alfredo Diez, and Thomas Nyström. "The bacterial universal stress protein: Function and regulation." In: Current Opinion in Microbiology 6.2 (2003), pp. 140145. DOI: $10.1016 / \mathrm{S} 1369-5274$ (03)00025-0.

[245] Vivek Anantharaman, Lakshminarayan M. Iyer, and L. Aravind. “Ter-dependent stress response systems: Novel pathways related to metal sensing, production of a nucleoside-like metabolite, and DNA-processing." In: Molecular BioSystems 8.12 (2012), pp. 3142-3165. DOI: 10.1039/c2mb25239b.

[246] Alexis H.K. Choi, Leyla Slamti, Fikri Y. Avci, Gerald B. Pier, and Tomás Maira-Litrán. "The pgaABCD locus of Acinetobacter baumannii encodes the production of poly- $\beta-1-6-\mathrm{N}$-acetylglucosamine, which is critical for biofilm formation." In: Journal of Bacteriology 191.19 (2009), pp. 5953-5963. DOI: 10.1128/JB.00647-09. 
[247] Jason W Reed and Graham C Walker. "The exoD Gene of Rhizobium meliloti encodes a novel function needed for Alfalfa nodule invasion." In: Journal of Bacteriology 173.2 (1991), pp. 664-677. DOI: $10.1128 / \mathrm{jb} .173 .2 .664-677.1991$.

[248] Yasser Y. Lenis, Mohammed A. Elmetwally, Juan G. MaldonadoEstrada, and Fuller W. Bazer. "Physiological importance of polyamines." In: Zygote 25.3 (2017), pp. 244-255. DOI: 10.1017/ S0967199417000120.

[249] A. J. Michael. "Biosynthesis of polyamines and polyamine-containing molecules." In: Biochemical Journal 473.15 (2016), pp. 23152329. DOI: 10.1042/BCJ20160185.

[250] Seiya Watanabe, Masaki Yamada, Iwao Ohtsu, and Keisuke Makino. " $\alpha$-Ketoglutaric semialdehyde dehydrogenase isozymes involved in metabolic pathways of D-glucarate, D-galactarate, and hydroxy-L-proline: Molecular and metabolic convergent evolution." In: Journal of Biological Chemistry 282.9 (2007), pp. 66856695. DOI: $10.1074 / \mathrm{jbc} . \mathrm{M} 611057200$.

[251] Michal Koblížek. "Ecology of aerobic anoxygenic phototrophs in aquatic environments." In: FEMS Microbiology Reviews 39.6 (2015), pp. 854-870. DOI: 10.1093/femsre/fuv032.

[252] Bork Ansgar Berghoff, Jens Glaeser, Aaron Mischa Nuss, Monica Zobawa, Friedrich Lottspeich, and Gabriele Klug. "Anoxygenic photosynthesis and photooxidative stress: A particular challenge for Roseobacter." In: Environmental Microbiology 13.3 (2011), pp. 775-791. DOI: 10.1111/j . 1462-2920 . 2010.02381. $\mathrm{x}$.

[253] Christopher T. Jurgenson, Tadhg P. Begley, and Steven E. Ealick. "The structural and biochemical foundations of thiamin biosynthesis." In: Annual Review of Biochemistry 78.1 (2009), pp. 569603. DOI: 10.1146/annurev . biochem. 78.072407.102340.

[254] Raymond Wightman and Peter A. Meacock. "The THI5 gene family of Saccharomyces cerevisiae: Distribution of homologues among the hemiascomycetes and functional redundancy in the aerobic biosynthesis of thiamin from pyridoxine." In: Microbiology 149.6 (2003), pp. 1447-1460. DOI: 10. 1099/mic . 0. 26194-0.

[255] Amy Haas Jenkins, Ghislain Schyns, Sébastien Potot, Guangxing Sun, and Tadhg P. Begley. "A new thiamin salvage pathway." In: Nature Chemical Biology 3.8 (2007), pp. 492-497. DOI: 10.1038/nchembio.2007.13.

[256] Sergio A. Sa nudo-Wilhelmy, Laura Gómez-Consarnau, Christopher Suffridge, and Eric A. Webb. "The role of B-vitamins in marine biogeochemistry." In: Annual Review of Marine Science 6.1 (2014), pp. 339-367. DOI: 10 . 1146/annurev-marine-120710100912. 
[257] Irene Wagner-Döbler et al. "The complete genome sequence of the algal symbiont Dinoroseobacter shibae: A hitchhiker's guide to life in the sea." In: ISME Journal 4.1 (2010), pp. 61-77. DOI: 10.1038/ismej .2009. 94.

[258] Semen A. Leyn, Inna A. Suvorova, Tatiana D. Kholina, Sofia S. Sherstneva, Pavel S. Novichkov, Mikhail S. Gelfand, and Dmitry A. Rodionov. "Comparative genomics of transcriptional regulation of methionine metabolism in proteobacteria." In: PLOS ONE 9.11 (2014). DOI: 10.1371/journal . pone. 0113714.

[259] Julio C Gonzáles, Katrina Peariso, James E Penner-hahn, and Rowena GMatthews. "Cobalamin-independent methionine synthase from Escherichia coli: A Zinc Metalloenzyme." In: Biochemistry 35.38 (1996), pp. 12228-12234.

[26o] Agnieszka Sekowska, Valérie Dénervaud, Hiroki Ashida, Karine Michoud, Dieter Haas, Akiho Yokota, and Antoine Danchin. "Bacterial variations on the methionine salvage pathway." In: BMC Microbiology 4 (2004), pp. 1-17. DOI: 10.1186/1471-21804-9.

[261] Eva Albers. "Metabolic characteristics and importance of the universal methionine salvage pathway recycling methionine from $5^{\prime}$-methylthioadenosine." In: IUBMB Life 61.12 (2009), pp. 11321142. DOI: 10.1002/iub. 278.

[262] Jeffrey A Hughes. "In vivo hydrolysis of S-adenosyl-L-methionine in Escherichia coli increases export of 5-methylthioribose." In: Canadian journal of microbiology 52.6 (2006), pp. 599-602. DOI: 10.1139/w06-008.

[263] R. A. Bender. "Regulation of the histidine utilization (Hut) system in bacteria." In: Microbiology and Molecular Biology Reviews 76.3 (2012), pp. 565-584. DOI: 10.1128/MMBR.00014- 12.

[264] Peep Palumaa. "Copper chaperones. the concept of conformational control in the metabolism of copper." In: FEBS Letters 587.13 (2013), pp. 1902-1910. DOI: 10.1016/j . febslet. 2013. Q5.019.

[265] Andre Zapun, James C A Bardwell, and Thomas E Creighton J. "The reactive and destabilizing disulfide bond of DsbA , a protein required for protein disulfide bond formation in vivo." In: Biochemistry 32.19 (1993), pp. 5083-5092. DOI: $10.1021 /$ bi00070a016.

[266] Melanie A. Adams and Zongchao Jia. "Structural and biochemical evidence for an enzymatic quinone redox cycle in Escherichia coli: Identification of a novel quinol monooxygenase." In: Journal of Biological Chemistry 280.9 (2005), pp. 8358-8363. DOI: 10 . $1074 / \mathrm{jbc}$. M412637200. 
[267] F. De Rienzo, R.R. Gabdoulline, M. C. Menziani, and R.C. Wade. "Blue copper proteins: A comparative analysis of their molecular interaction properties." In: Protein Science 9.8 (2000), pp. 14391454. DOI: $10.1110 / \mathrm{ps} .9 .8 .1439$.

[268] Octavian Călinescu, Cristina Paulino, Werner Kühlbrandt, and Klaus Fendler. "Keeping it simple - Transport mechanism and $\mathrm{pH}$ regulation in $\mathrm{Na}^{+} / \mathrm{H}^{+}$exchangers." In: J Biol Chem 289.19 (2014), pp. 13168-76. DOI: 10.1074/jbc.M113. 542993.

[269] B. Aral and P. Kamoun. "The proline biosynthesis in living organisms." In: Amino Acids 13.3-4 (1997), pp. 189-217. DOI: 10. 1007/BFQ1372588.

[270] E A Galinski. "Osmoadaptation in bacteria." In: Advances in Microbial Physiology 37 (1995), pp. 273-328. DOI: 10 . 1016/S00652911 (08) 60148-4.

[271] Ukti S Amin, Timothy D Lash, and Brian J Wilkinson. "Proline betaine is a highly effective osmoprotectant for Staphylococcus aureus." In: Archives of Microbiology 163.2 (1995), pp. 138-142. DOI: $10.1007 / \mathrm{BF} 00381788$.

[272] Christopher A. Dunn, Suzanne F. O'Handley, David N. Frick, and Maurice J. Bessman. "Studies on the ADP-ribose pyrophosphatase subfamily of the nudix hydrolases and tentative identification of $\operatorname{trgB}$, a gene associated with tellurite resistance." In: Journal of Biological Chemistry 274.45 (1999), pp. 32318-32324. DOI: $10.1074 / \mathrm{jbc} .274 .45 .32318$.

[273] Suzanne F. O'Handley, David N. Frick, Christopher A. Dunn, and Maurice J. Bessman. "Orf186 represents a new member of the nudix hydrolases, active on adenosine (5')triphospho( $\left.5^{\prime}\right)$ adenosine, ADP-ribose, and NADH." In: Journal of Biological Chemistry 273.6 (1998), pp. 3192-3197. DOI: 10.1074/ jbc . 273.6 . 3192.

[274] Elaine L. Jacobson, Daniel Cervantes-Laurean, and Myron K. Jacobson. "Glycation of proteins by ADP-ribose." In: Molecular and Cellular Biochemistry 138.1-2 (1994), pp. 207-212. DOI: 10 . $1007 / B F 00928463$.

[275] Corinna Rademacher, Marie Christine Hoffmann, Jan Wilm Lackmann, Roman Moser, Yvonne Pfänder, Silke Leimkühler, Franz Narberhaus, and Bernd Masepohl. "Tellurite resistance gene $\operatorname{trg} B$ confers copper tolerance to Rhodobacter capsulatus." In: BioMetals 25.5 (2012), pp. 995-1008. DOI: 10 . 1007/s10534Q12-9566-2.

[276] Ivan Erill, Susana Campoy, and Jordi Barbé. "Aeons of distress: An evolutionary perspective on the bacterial SOS response." In: FEMS Microbiology Reviews 31.6 (2007), pp. 637-656. DOI: $10.1111 / \mathrm{j} .1574-6976.2007 .00082 . \mathrm{x}$. 
[277] Evelyn Barth, Katherine V. Gora, Katharina M. Gebendorfer, Florian Settele, Ursula Jakob, and Jeannette Winter. "Interplay of cellular cAMP levels, oS activity and oxidative stress resistance in Escherichia coli." In: Microbiology 155.5 (2009), pp. 16801689. DoI: $10.1099 / \mathrm{mic} .0 .026021-0$.

[278] Takuya Kasai, Atsushi Kouzuma, and Kazuya Watanabe. “CpdA is involved in amino acid metabolism in Shewanella oneidensis MR-1." In: Bioscience, Biotechnology, and Biochemistry (2017). DOI: 10.1080/09168451.2017.1413326.

[279] Iralis López-Villamizar, Alicia Cabezas, Rosa María Pinto, José Canales, Jo ao Meireles Ribeiro, José Carlos Cameselle, and María Jesús Costas. "The characterization of Escherichia coli $\mathrm{CpdB}$ as a recombinant protein reveals that, besides Having the expected 3-nucleotidase and 2,3-cyclic mononucleotide phosphodiesterase activities, it is also active as cyclic dinucleotide phosphodiesterase." In: PLOS ONE 11.6 (2016), pp. 1-22. DOI: 10.1371/journal . pone. 0157308.

[280] Emilykate Mcdonough, Heather Kamp, and Andrew Camilli. "Vibrio cholerae phosphatases required for the utilization of nucleotides and extracellular DNA as phosphate sources." In: Molecular Microbiology 99.3 (2016), pp. 453-469. DOI: 10.1111/ mmi. 13128.

[281] Konrad Trülzsch, Andreas Roggenkamp, Cosima Pelludat, Alexander Rakin, Christoph A Jacobi, and Jürgen Heesemann. "Cloning and characterization of the gene encoding periplasmic 2',3'-cyclic phosphodiesterase of Yersinia enterocolitica O:8." In: Microbiology 147 (2001), pp. 203-213. DOI: 10 . 1099/00221287147-1-203.

[282] Hans Jörg Kunte. “Osmoregulation in bacteria: Compatible solute accumulation and osmosensing." In: Environmental Chemistry 3.2 (2006), pp. 94-99. DOI: 10.1071/EN06016.

[283] Anne Carlijn Alderkamp, Marion Van Rijssel, and Henk Bolhuis. "Characterization of marine bacteria and the activity of their enzyme systems involved in degradation of the algal storage glucan laminarin." In: FEMS Microbiology Ecology 59.1 (2007), pp. 108-117. DOI: 10.1111/j .1574-6941.2006.00219.x.

[284] Roberta Leonardi, Yong Mei Zhang, Charles O. Rock, and Suzanne Jackowski. "Coenzyme A: Back in action." In: Progress in Lipid Research 44 (2005), pp. 125-153. DOI: 10. 1016/j . plipres. 2005. 04.001 .

[285] Eugene V. Koonin. “Orthologs, paralogs, and evolutionary genomics." In: Annual Review of Genetics 39.1 (2005), pp. 309-338. DOI: 10.1146/annurev.genet. 39.073003.114725.

[286] Leonidas Salichos and Antonis Rokas. "Inferring ancient divergences requires genes with strong phylogenetic signals." In: $\mathrm{Na}$ ture 497.7449 (2013), pp. 327-331. DOI: 10.1038/nature12130. 
[287] Dana E. Hunt, Lawrence A. David, Dirk Gevers, Sarah E. Preheim, Eric J. Alm, and Martin F. Polz. "Resource partitioning and sympatric differentiation among closely related bacterioplankton." In: Science 320.5879 (2008), pp. 1681-1685. DOI: 10 . 1126/science. 1157890.

[288] Duccio Medini, Claudio Donati, Hervé Tettelin, Vega Masignani, and Rino Rappuoli. "The microbial pan-genome." In: Current Opinion in Genetics and Development 15.6 (2005), pp. 589-594. DOI: $10.1016 / \mathrm{j}$.gde.2005.09.006.

[289] Todd J. Treangen and Eduardo P C Rocha. "Horizontal transfer, not duplication, drives the expansion of protein families in prokaryotes." In: PLoS Genetics 7.1 (2011). DOI: 10 . 1371 / journal.pgen. 1001284.

[290] H. Tettelin et al. "Genome analysis of multiple pathogenic isolates of Streptococcus agalactiae: implications for the microbial "pan-genome"." In: Proceedings of the National Academy of Sciences 102.39 (2005), pp. 13950-13955. DOI: 10.1073 / pnas . Q506758102.

[291] Hervé Tettelin, David Riley, Ciro Cattuto, and Duccio Medini. "Comparative genomics: the bacterial pan-genome." In: Current Opinion in Microbiology 11.5 (2008), pp. 472-477. DOI: 10.1016/ j.mib.2008.09.006.

[292] Mark Eppinger et al. "Genome sequences of the biotechnologically important Bacillus megaterium Strains QM B1551 and DSM319." In: Journal of Bacteriology 193.16 (2011), pp. 4199-4213. DOI: $10.1128 / \mathrm{JB} .00449-11$.

[293] Tristan Lefébure and Michael J. Stanhope. "Evolution of the core and pan-genome of Streptococcus: Positive selection, recombination, and genome composition." In: Genome Biology 8.5 (2007), pp. 1-17. DOI: $10.1186 / \mathrm{gb}-2007-8-5-\mathrm{r} 71$.

[294] Francesca Bottacini, Duccio Medini, Angelo Pavesi, Francesca Turroni, Elena Foroni, David Riley, Vanessa Giubellini, Hervé Tettelin, Douwe Van Sinderen, and Marco Ventura. "Comparative genomics of the genus Bifidobacterium." In: Microbiology 156.11 (2010), pp. 3243-3254. DOI: 10.1099/mic.0.039545-0.

[295] Adam C. Martiny, Kathleen Treseder, and Gordon Pusch. "Phylogenetic conservatism of functional traits in microorganisms." In: ISME Journal 7.4 (2013), pp. 830-838. DOI: 10.1038/ismej . 2012.160 .

[296] Konstantinos T Konstantinidis and James M Tiedje. "Trends between gene content and genome size in prokaryotic species with larger genomes." In: Proceedings of the National Academy of Sciences of the United States of America 9 (2004), pp. 3160-3165. DOI: $10.1073 /$ pnas. 0308653100 . 
[297] Antony T. Vincent, Mélanie V. Trudel, Luca Freschi, Vandan Nagar, Cynthia Gagné-Thivierge, Roger C. Levesque, and Steve J. Charette. "Increasing genomic diversity and evidence of constrained lifestyle evolution due to insertion sequences in Aeromonas salmonicida." In: BMC Genomics 17.44 (2016). DOI: 10.1186/ s12864-016-2381-3.

[298] Federico M Lauro et al. "The genomic basis of trophic strategy in marine bacteria." In: Proceedings of the National Academy of Sciences of the United States of America 106.37 (2009), pp. 1552733. DOI: $10.1073 /$ pnas. 0903507106.

[299] Yuri I. Wolf and Eugene V. Koonin. "Genome reduction as the dominant mode of evolution." In: BioEssays 35.9 (2013), pp. 829837. DOI: $10.1002 /$ bies. 201300037.

[300] Ariane Toussaint and Mick Chandler. "Prokaryote genome fluidity: Toward a system approach of the mobilome." In: Bacterial Molecular Networks. Ed. by Jacques van Helden, Ariane Toussaint, and Denis Thieffry. New York: Humana Press, 2012. Chap. 4, pp. 57-80. ISBN: 978-1-61779-360-8.

[301] Dan I. Andersson and Diarmaid Hughes. "Gene amplification and adaptive evolution in bacteria." In: Annual Review of Genetics 43.1 (2009), pp. 167-195. DOI: 10 . 1146/annurev-genet-102108134805 .

[302] Pavel Drevinek, Adam Baldwin, Laurens Lindenburg, Lovleen Tina Joshi, Angela Marchbank, Sarka Vosahlikova, Christopher G. Dowson, and Eshwar Mahenthiralingam. "Oxidative stress of Burkholderia cenocepacia induces insertion sequence-mediated genomic rearrangements that interfere with macrorestrictionbased genotyping." In: Journal of Clinical Microbiology 48.1 (2010), pp. 34-40. DOI: 10.1128/JCM.01433-09.

[303] Colin J. Coros, Carol Lyn Piazza, Venkata R. Chalamcharla, Dorie Smith, and Marlene Belfort. "Global regulators orchestrate group II intron retromobility." In: Molecular Cell 34.2 (2009), pp. 250-256. DOI: 10.1016/j . molcel .2009.03.014.

[304] Cécile Pasternak, Bao Ton-Hoang, Geneviève Coste, Adriana Bailone, Michael Chandler, and Suzanne Sommer. "Irradiationinduced Deinococcus radiodurans genome fragmentation triggers transposition of a single resident insertion sequence." In: PLoS Genetics 6.1 (2010), pp. 1-10. DOI: 10.1371/ journal . pgen . 1000799.

[305] Elena Casacuberta and Josefa González. "The impact of transposable elements in environmental adaptation." In: Molecular Ecology 22.6 (2013), pp. 1503-1517. DOI: 10.1111/mec. 12170.

[306] Daniel M. Stoebel and Charles J. Dorman. "The effect of mobile element IS10 on experimental regulatory evolution in Escherichia coli." In: Molecular Biology and Evolution 27.9 (2010), pp. 2105-2112. DOI: 10.1093/molbev/msq101. 
[307] Alexandre M. Anesio and Christopher M. Bellas. "Are low temperature habitats hot spots of microbial evolution driven by viruses?" In: Trends in Microbiology 19.2 (2011), pp. 52-57. DOI: 10.1016/j.tim.2010.11.002.

[308] Ildefonso Cases, Victor De Lorenzo, and Christos A Ouzounis. "Transcription regulation and environmental adaptation in bacteria." In: Trends in Microbiology 11.6 (2003), pp. 248-253. DOI: 10. 1016/S0966-842X(03)00103-3.

[309] Pablo A. Nu nez, Héctor Romero, Marisa D. Farber, and Eduardo P C Rocha. "Natural selection for operons depends on genome size." In: Genome Biology and Evolution 5.11 (2013), pp. 22422254. DOI: 10 . 1093/gbe/evt 174 .

[310] Llyd E. Wells and Jody W. Deming. "Modelled and measured dynamics of viruses in Arctic winter sea-ice brines." In: Environmental Microbiology 8.6 (2006), pp. 1115-1121. DOI: 10.1111/ j. $1462-2920.2006 .00984 . x$.

[311] Jeff S. Bowman. "The relationship between sea ice bacterial community structure and biogeochemistry: A synthesis of current knowledge and known unknowns." In: 3 (2015). DOI: 10.12952/ journal. elementa.000072.

[312] Martin T. Croft, Andrew D. Lawrence, Evelyne Raux-Deery, Martin J. Warren, and Alison G. Smith. "Algae acquire vitamin B12 through a symbiotic relationship with bacteria." In: Nature 438.7064 (2005), pp. 90-93. DOI: 10.1038/nature04056.

[313] Benjamin R.K. Roller, Steven F. Stoddard, and Thomas M. Schmidt. "Exploiting rRNA operon copy number to investigate bacterial reproductive strategies." In: Nature Microbiology 1.September (2016). DOI: $10.1038 /$ nmicrobiol.2016.160.

[314] A. Boetius et al. "Export of algal biomass from the melting Arctic sea ice." In: Science 339.6126 (2013), pp. 1430-1432. DOI: 10.1126/science. 1231346.

[315] Dorte Haubjerg Søgaard, Morten Kristensen, Søren Rysgaard, Ronnie Nøhr Glud, Per Juel Hansen, and Karen Marie Hilligsøe. "Autotrophic and heterotrophic activity in Arctic first-year sea ice: Seasonal study from Malene Bight, SW Greenland." In: Marine Ecology Progress Series 419 (2010), pp. 31-45. Dor: 10. 3354/ meps08845.

[316] Richard J. Geider and Julie La Roche. "Redfield revisited: Variability of C:N:P in marine microalgae and its biochemical basis." In: European Journal of Phycology 37.1 (2002), pp. 1-17. DOI: 10.1017/S0967026201003456.

[317] Alasdair M. Cook and Karin Denger. "Dissimilation of the C2 sulfonates." In: Archives of Microbiology 179.1 (2002), pp. 1-6. DOI: $10.1007 / \mathrm{s} 00203-002-0497-0$. 
[318] Karin Denger, Jürgen Ruff, David Schleheck, and Alasdair M. Cook. "Rhodococcus opacus expresses the xsc gene to utilize taurine as a carbon source or as a nitrogen source but not as a sulfur source." In: Microbiology 150.6 (2004), pp. 1859-1867. DOI: 10.1099/mic.0.27077-0.

[319] Robert C. Fahey. "Glutathione analogs in prokaryotes." In: Biochimica et Biophysica Acta - General Subjects 1830.5 (2013), pp. 31823198. DoI: $10.1016 / \mathrm{j}$. bbagen. 2012.10 .006 .

[320] Johannes Hartl, Patrick Kiefer, Fabian Meyer, and Julia A. Vorholt. "Longevity of major coenzymes allows minimal de novo synthesis in microorganisms." In: Nature Microbiology 2.May (2017). DOI: $10.1038 /$ nmicrobiol.2017.73.

[321] Qiang Zheng, Michal Koblížek, Thomas J Beatty, and Nianzhi Jiao. "Evolutionary divergence of marine aerobic anoxygenic phototrophic bacteria as seen from diverse organisations of their photosynthesis gene clusters." In: Advances in Botanical Research Volume 66 - Genome Evolution of Photosynthetic Bacteria. Ed. by Jean-pierre Jacquot, Pierre Gadal, and J. Thomas Beatty. Amsterdam: Elsevier Ltd., 2013. Chap. 12, pp. 359-383. ISBN: 9780123979230. DOI: 10.1016/B978-0-12-397923-0.00012-6.

[322] James N. Sturgis and Robert A. Niederman. “Organization and assembly of light-harvesting complexes in the purple bacterial membrane." In: Advances in Photosynthesis and Respiration - Volume 28: The Purple Phototrophic Bacteria. Ed. by Neil C. Hunter, Fevzi Daldal, Marion C. Thurnauer, and Thomas J. Beatty. 1. Springer, 2009. Chap. 14, pp. 253-273. ISBN: 978-1-4020-8814-8.

[323] Merja Suutari and Siino Laakso. "Microbial fatty acids and thermal adaptation." In: Critical Reviews in Microbiology 20.4 (1994), pp. 285-328. DOI: 10.3109/10408419409113560.

[324] Michael Hügler and Stefan M. Sievert. "Beyond the calvin cycle: Autotrophic carbon fixation in the ocean." In: Annual Review of Marine Science 3.1 (2011), pp. 261-289. DoI: 10.1146/annurevmarine-120709-142712.

[325] Thibaud Taillefumier, Anna Posfai, Yigal Meir, and Ned S Wingreen. "Microbial consortia at steady supply." In: eLife 6 (2017). Ed. by Carl T Bergstrom, e22644. Dor: 10.7554/eLife. 22644.

[326] George N. Somero. "Adaptation of enzymes to temperature: Searching for basic "strategies"." In: Comparative Biochemistry and Physiology - B Biochemistry and Molecular Biology 139 (2004), pp. 321-333. DOI: 10.1016/j . cbpc. 2004.05.003.

[327] Raghu Prasad Rao Metpally and Boojala Vijay B. Reddy. “Comparative proteome analysis of psychrophilic versus mesophilic bacterial species: Insights into the molecular basis of cold adaptation of proteins." In: BMC Genomics 10.11 (2009). DOI: 10 . 1186/1471-2164-10-11. 
[328] Alexandra Moura, Michael A. Savageau, and Rui Alves. "Relative amino acid composition signatures of organisms and environments." In: PLOS ONE 8.10 (2013), e77319. DOI: 10.1371/ journal . pone. 0077319 .

[329] Emmanuele De Vendittis, Immacolata Castellano, Roberta Cotugno, Maria Rosaria Ruocco, Gennaro Raimo, and Mariorosario Masullo. "Adaptation of model proteins from cold to hot environments involves continuous and small adjustments of average parameters related to amino acid composition." In: Journal of Theoretical Biology 250.1 (2008), pp. 156-171. DOI: 10.1016/j . jtbi.2007.09.006.

[330] David K. Smith, Predrag Radivojac, Zoran Obradovic, A. Keith Dunker, and Guang Zhu. "Improved amino acid flexibility parameters." In: Protein Science 12.5 (2003), pp. 1060-1072. DOI: $10.1110 / \mathrm{ps} .0236203$.

[331] Nikolaj Tindbaek, Allan Svendsen, Peter Rahbek Oestergaard, and Henriette Draborg. "Engineering a substrate-specific coldadapted subtilisin." In: Protein Engineering, Design and Selection 17.2 (2004), pp. 149-156. DOI: 10.1093/protein/gzh019.

[332] Shinsuke Maki, Mizuho Yoneta, and Yasuhiro Takada. "Two isocitrate dehydrogenases from a psychrophilic bacterium, Colwellia psychrerythraea." In: Extremophiles 10.3 (2006), pp. 237-249. DOI: $10.1007 / \mathrm{s} 00792-005-0493-9$.

[333] Hye Yeon Koh, Hyun Park, Jun Hyuck Lee, Se Jong Han, Young Chang Sohn, and Sung Gu Lee. "Proteomic and transcriptomic investigations on cold-responsive properties of the psychrophilic Antarctic bacterium Psychrobacter sp. PAMC 21119 at subzero temperatures." In: Environmental Microbiology 19.2 (2017), pp. 628644. DOI: 10.1111/1462-2920.13578.

[334] Federico M. Lauro, Khiem Tran, Alessandro Vezzi, Nicola Vitulo, Giorgio Valle, and Douglas H. Bartlett. "Large-scale transposon mutagenesis of Photobacterium profundum SS9 reveals new genetic loci important for growth at low temperature and high pressure." In: Journal of Bacteriology 190.5 (2008), pp. 1699-1709. DOI: 10.1128/JB.01176-07.

[335] James J. Foti, Lyle A. Simmons, Penny J. Beuning, and Graham C. Walker. "Signal transduction in the Escherichia coli SOS response." In: Handbook of Cell Signaling. Second Edition. Elsevier Inc., 2010. Chap. 258, pp. 2127-2136. ISBN: 9780123741455. DOI: 10.1016/B978-0-12-374145-5.00258-8.

[336] L. Marsh and G. C. Walker. "Cold sensitivity induced by overproduction of UmuDC in Escherichia coli." In: Journal of Bacteriology 162.1 (1985), pp. 155-161. 
[337] Carole L. Linster, Emile Van Schaftingen, and Andrew D. Hanson. "Metabolite damage and its repair or pre-emption." In: Nature Chemical Biology 9.2 (2013), pp. 72-80. Dor: 10.1038/ nchembio. 1141.

[338] Tatiana V. Karpinets et al. "Conserved synteny at the protein family level reveals genes underlying Shewanella species' cold tolerance and predicts their novel phenotypes." In: Functional and Integrative Genomics 10.1 (2010), pp. 97-110. DOI: 10.1007/ s10142-009-0142-y.

[339] K. Limsuwun and P. G. Jones. "Spermidine acetyltransferase is required to prevent spermidine toxicity at low temperatures in Escherichia coli." In: Journal of Bacteriology 182.19 (2000), pp. 53735380. DOI: $10.1128 /$ JB. $182.19 .5373-5380.2000$.

[340] Jon M Kaguni. "Replication initiation at the Escherichia coli chromosomal origin." In: Current Opinion in Chemical Biology 15.5 (2011), pp. 606-613. DOI: 10.1016/j . cbpa.2011.07.016.

[341] Ricardo Cavicchioli. "Microbial ecology of Antarctic aquatic systems." In: Nature Reviews Microbiology 13.11 (2015), pp. 691706. DOI: $10.1038 /$ nrmicro3549.

[342] B. J. Tindall. "Prokaryotic diversity in the Antarctic: The tip of the iceberg." In: Microbial Ecology 47.3 (2004), pp. 271-283. DOI: $10.1007 / \mathrm{s} 00248-003-1050-7$.

[343] Liisa B. Koski and G. Brian Golding. "The closest BLAST hit is often not the nearest neighbor." In: Journal of Molecular Evolution 52.6 (2001), pp. 540-542. DOI: 10.1007/s002390010184.

[344] Seungtai Yoon, Zhenyu Xuan, Vladimir Makarov, Kenny Ye, and Jonathan Sebat. "Sensitive and accurate detection of copy number variants using read depth of coverage." In: Genome Research 19.9 (2009), pp. 1586-1592. DOI: 10.1101/gr . 092981. 109. 


\section{DECLARATION}

I intend to write a doctoral thesis on the topic Distribution and ecological characteristics of members of the Roseobacter group at GeorgAugust-Universität Göttingen. I will be supervised by Prof. Dr. Rolf Daniel.

I hereby declare that:

1. the opportunity to work on the aforementioned doctoral thesis project was not arranged commercially. Especially, I did not engage any organisation which searches for doctoral thesis supervisors or which will entirely or partly carry out my examination duties against payment;

2. I have only accepted and will only accept the assistance of third parties in so far as it is scientifically justifiable and acceptable in regards to the examination regulations. Especially, all parts of the dissertation will be written by myself; I have not accepted and will not accept impermissible help from other parties neither for money nor for free;

3. I will observe the Statute of the Georg-August-University Göttingen for ensuring good scientific practice;

4. I have not applied for corresponding doctoral degree procedures at any other university in Germany or abroad; the submitted doctoral thesis or parts thereof were not used in another doctoral degree procedure.

I am aware that incorrect information precludes the admission to doctoral studies resp. may later on lead to the discontinuation of the doctoral degree procedures or to the revocation of the doctoral degree.

Göttingen, 24/06/2020

Florian Lenk 



\title{
CURRICULUM VITAE
}

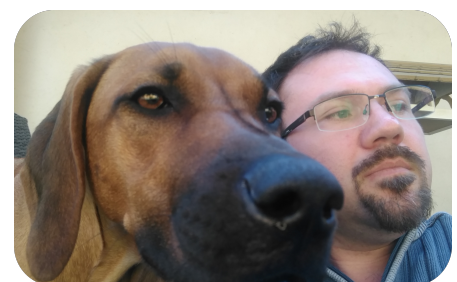

\author{
Florian Lenk \\ * 01.11.1986 \\ $\triangle$ Quantzstraße $32 \mathrm{a}$ \\ 37127 Scheden \\ flenk@gwdg.de
}

\author{
since 02/2015 doctorate in the Dept. of Genomic and Applied \\ Microbiology, Faculty of Biology and Psychology, \\ Georg-August University Göttingen \\ 10/2009 - M.Sc. in Microbiology and Biochemistry, \\ 09/2014 Georg-August University Göttingen \\ 10/2006 - B.Sc. in Molecular Medicine, Georg-August \\ 09/2009 University Göttingen \\ 07/1999 - university-entrance diploma at the Grotefend \\ o6/2006 Gymnasium Hann. Münden
}





\section{COLOPHON}

This document was typeset using the typographical look-and-feel classicthesis developed by André Miede. The style was inspired by Robert Bringhurst's seminal book on typography "The Elements of Typographic Style". classicthesis is available for both $\mathrm{ET}_{\mathrm{E}} \mathrm{X}$ and $\mathrm{LYX}$ :

https://bitbucket.org/amiede/classicthesis/

Final Version as of June 24, 2020 (classicthesis version 4.2). 